\title{
Modulation of lateral membrane tension and SNARE-mediated single vesicle fusion on pore spanning membranes
}

\author{
Dissertation \\ for the award of the degree \\ Doctor rerum naturalium \\ of the Georg-August-Universität Göttingen \\ within the doctoral program \\ Chemistry of the Georg-August University School of Science (GAUSS)
}

submitted by

Jan Wilhelm Kuhlmann

from Düsseldorf

Göttingen 2017 



\title{
Modulation of lateral membrane tension and SNARE-mediated single vesicle fusion on pore spanning membranes
}

\author{
Dissertation \\ for the award of the degree \\ Doctor rerum naturalium \\ of the Georg-August-Universität Göttingen \\ within the doctoral program \\ Chemistry of the Georg-August University School of Science (GAUSS)
}

submitted by

Jan Wilhelm Kuhlmann

from Düsseldorf

Göttingen 2017 


\section{Members of the Thesis Committee}

Prof. Dr. Claudia Steinem,

Institut für Organische und Biomolekulare Chemie,

Georg-August-Universität Göttingen

Dr. Iwan Schaap,

Institute of Biological Chemistry, Biophysics and Bioengineering,

Heriot-Watt University

\section{Members of the Examination Board}

Referee: Prof. Dr. Claudia Steinem,

Institut für Organische und Biomolekulare Chemie,

Georg-August-Universität Göttingen

$2^{\text {nd }}$ referee: Prof. Dr. Tim Salditt,

Institut für Röntgenphysik,

Georg-August-Universität Göttingen

\section{Further members of the Examination Board}

Prof. Dr. Bert de Groot,

Institute for Computational Biolmolecular Dynamics,

Max Planck Institute for Biophysical Chemistry Göttingen

Prof. Dr. Burkhard Geil,

Institut für Physikalische Chemie,

Georg-August-Universität Göttingen

Prof. Dr. Michael Meinecke,

Institut für Zelluläre Biochemie,

Universitätsmedizin Göttingen

Prof. Dr. Silvio Rizzoli,

Institut für Neuro- und Sinnesphysiologie,

Universitätsmedizin Göttingen

\section{Date of oral examination}

$12^{\text {th }}$ July 2017 


\section{Declaration}

I, Jan Wilhelm Kuhlmann, hereby certify that my doctoral thesis entitled "Modulation of lateral membrane tension and SNARE-mediated single vesicle fusion on pore-spanning membranes" has been written independently and with no other sources and aids than quoted.

Göttingen, 2017

Jan Wilhelm Kuhlmann 

"Es kommt nicht darauf an, mit dem Kopf durch die Wand zu rennen, sondern mit den Augen die Tür zu finden."

Werner von Siemens 

Abstract. Synaptic vesicle fusion is driven by soluble N-ethylmaleimide-sensitivefactor attachment receptors (SNAREs) but is also regulated by physical properties of the membrane like curvature and tension. Using artificial SNARE containing prestressed pore-spanning membranes (PSMs) comprised of supported membranes (s-PSM) and freestanding membranes (f-PSM), the influence of lateral tension on SNARE-induced fusion, as well as dynamic aspects of vesicle docking and fusion on the two distinct membrane parts can be investigated.

PSMs were prepared on porous substrates functionalized with binary self-assembled monolayers (SAM) composed of 6-mercaptohexanol (6-MH) and cholesterylpolyethylenoxythiol (CPEO3). Varying the amount of CPEO3 in the SAM allowed to modulate the lateral tension in the PSM. The tensions ranged from $1.4 \mathrm{mN} \mathrm{m}^{-1}$ for $0 \mathrm{~mol} \%$ CPEO3 to $4.5 \mathrm{mN} \mathrm{m}^{-1}$ for $35 \mathrm{~mol} \%$ CPEO3 in the SAM. With increasing lateral tension, i.e. higher CPEO3 surface concentrations, the membrane stability decreased resulting in rupturing of the freestanding membranes.

To investigate the influence of the lateral tension on SNARE-mediated fusion and to compare fusion on s-PSM and f-PSM, vesicular-SNARE proteoliposomes were fused with target-SNARE containing PSMs. The PSMs were prepared on pure 6MH SAMs as the membranes composed of a physiological lipid mixture, which was essential for efficient fusion, were unstable on SAMs containing CPEO3. Docking of the liposomes could be observed on the s-PSM and f-PSM but was two times more likely on the s-PSM. The s-PSM docked liposomes were completely immobile, while those on the f-PSMs were mobile with a mean diffusion coefficient of $D=0.42 \mu \mathrm{m}^{2} \mathrm{~s}^{-1}$. In comparison to a single SNARE transmembrane domain, the diffusivity of the f-PSM docked vesicles were eight times slower indicating a close contact of the membranes induced by tight SNARE-complexes. The immobile s-PSM docked vesicles probably arise from interactions between the SNAREs and the support. The docking time distributions on s-PSMs and f-PSMs were both in the range of seconds indicating that the higher mobility of the vesicles and the lateral membrane tension in the f-PSM do not significantly alter the fusion kinetics. The fusion kinetics were also unaffected by the size of the fusing vesicle as small $(90 \mathrm{~nm})$ and large $(240 \mathrm{~nm})$ vesicles exhibited comparable docking times. The shape of the docking time distributions indicated a two-step process, which can be either interpreted with the assembly of two SNARE complexes or with a kinetic intermediate. 
Zusammenfassung. Fusion synaptischer Vesikel wird durch soluble N-ethylmaleimidesensitive-factor attachment receptors (SNAREs) katalysiert, jedoch auch durch Membrankrümmung und -spannung gesteuert. Artifizielle vorgespannte porenüberspannende Membranen (PSMs), die sich durch unterstützte (s-PSMs) und freitragende Membranen (f-PSMs) auszeichnen, ermöglichen es, den Einfluss von Lateralspannung auf die SNARE-induzierte Fusion als auch dynamische Aspekte des Vesikeldockens und -fusionierens in den beiden Membranbereichen zu untersuchen.

PSMs wurden auf porösen Substraten, die mit einer binären selbst-assemblierten Monoschicht (SAM) bestehend aus 6-mercaptohexanol (6-MH) und cholesterylpolyethylenoxythiol (CPEO3) funktionalisierte wurden, präpariert. Durch Variation des CPEO3 Anteils im SAM konnte die Lateralspannung der PSMs verändert werden. Es konnten Lateralspannungen von $1.4 \mathrm{mN} \mathrm{m}^{-1}$ für $0 \mathrm{~mol} \% \mathrm{CPEO} 3$ und $4.5 \mathrm{mN} \mathrm{m}^{-1}$ für $35 \mathrm{~mol} \%$ CPEO3 im SAM bestimmt werden. Mit zunehmender Lateralspannung, d.h. höherem CPEO3 Anteil, verringerte sich jedoch auch die Stabilität der PSMs, was sich im Reißen der Membranen äußerte.

Um den Einfluss von Lateralspannung auf die SNARE-induzierte Fusion zu untersuchen und Fusion auf s-PSMs und f-PSMs zu vergleichen, wurden vesicular-SNARE Proteoliposomen mit target-SNARE enthaltenden PSMs fusioniert. Die PSMs wurden auf reinen 6-MH SAMs präpariert, da die aus einer physiologischen Lipidmischung bestehenden Membranen, die für die Fusion notwendig war, auf SAMs mit CPEO3 nicht stabil waren. Docken der Liposomen wurde auf s-PSMs und f-PSMs beobachtet. Die s-PSM gedockten Liposomen waren vollständig immobil, während f-PSM gedockte Liposomen mit einem Diffusionskoeffizienten von $D=0.42 \mu \mathrm{m}^{2} \mathrm{~s}^{-1}$ mobil waren. Im Vergleich zu einer einzelnen SNARE-Transmembrandomäne war die Diffusivität der f-PSM gedockten Liposomen achtmal langsamer, was auf einen engen Kontakt der beiden Membranen hinweist. Die Immobilität der s-PSM gedockten Liposomen ist wahrscheinlich eine Folge der starken Wechselwirkung zwischen den SNAREs und der Substratoberfläche. Die Dockingzeitverteilungen auf den s-PSMs und f-PSMs lag jeweils im Sekundenbereich und verdeutlich damit, dass kein nennenswerter Einfluss der höheren Mobilität und der Lateralspannung in der f-PSM auf die Fusion zu beobachten ist. Die Fusionskinetiken waren ebenfalls unbeeinflusst von der Größe der Liposomen, da kleine $(90 \mathrm{~nm})$ und große $(240 \mathrm{~nm})$ Vesikel vergleichbare Dockingzeiten aufwiesen. Die Form der Dockingzeitverteilung deutete zudem auf einen Zwei-Schritt Prozess hin, der mit der Assemblierung von zwei SNARE-Komplexen oder einer kinetischen Zwischenstufe erklärt werden kann. 


\section{Contents}

$\begin{array}{lll}1 & \text { Introduction } & 1\end{array}$

1.1 Membrane tension as a regulator for exocytosis . . . . . . . . . . . . 1

1.2 SNARE-mediated membrane fusion . . . . . . . . . . . . . . . . . 2

1.3 In vitro assays for membrane fusion . . . . . . . . . . . . . . . . . . 5

1.3 .1 Ensemble vesicle fusion assays . . . . . . . . . . . . . . 5

1.3 .2 Single vesicle fusion assays . . . . . . . . . . . . . . . . 6

1.3.3 SNAREs in in vitro fusion assays . . . . . . . . . . . . . . . . . 9

\begin{tabular}{lll}
\hline 2 & Scope & 11
\end{tabular}

3 Materials and Methods 13

3.1 Materials . . . . . . . . . . . . . . . . . . . . . . 13

3.1 .1 Porous substrates $\ldots \ldots \ldots$. . . . . . . . . . . . . . 13

3.1 .2 Lipids . . . . . . . . . . . . . . . . . . . . . . . 14

$3.1 .3 \quad$ Fluorescence probes . . . . . . . . . . . . . . . . . . . . 15

3.1 .4 SNARES . . . . . . . . . . . . . . . . . . . 15

3.2 Preparative methods $\ldots \ldots \ldots \ldots$. . . . . . . . . . . . . 18

3.2 .1 Giant unilamellar vesicles . . . . . . . . . . . . . . . . . . 18

3.2 .2 Isolation and purification of SNAREs . . . . . . . . . . . . . . . 19

3.2 .3 Reconstitution of SNAREs into liposomes . . . . . . . . . . . 25

3.2.4 Bulk SNARE activity experiments . . . . . . . . . . . . . . . 26

3.2 .5 Pore-spanning membranes (PSMs) . . . . . . . . . . . . . 26

$3.2 .6 \quad$ Density gradient centrifugation . . . . . . . . . . . . . 27

3.2 .7 Ion exchange chromatography . . . . . . . . . . . . . . . . . . 28

$3.2 .8 \quad$ SDS-polyacrylamide gel electrophoresis . . . . . . . . . . . . . . 29

3.3 Biophysical methods . . . . . . . . . . . . . . . . . . . . . . 30

3.3 .1 Spinning disc confocal microscopy (SDCM) . . . . . . . . . . . . 30

3.3.2 Atomic force microscopy (AFM) . . . . . . . . . . . . . . 32 
3.3.3 Polarization modulation infrared reflection-absorption spectroscopy

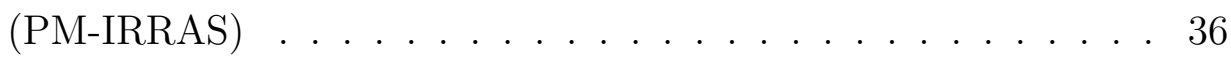

3.3 .4 Contact angle measurements . . . . . . . . . . . . . 38

4 Results: Lateral membrane tension of pore-spanning membranes 41

4.1 Wettability of 6-MH/CPEO3 self-assembled monolayers on gold . . . . 42

4.2 Chemical analysis of 6-MH/CPEO3 self-assembled monolayers on gold. 44

$4.3 \quad$ Stability of pore-spanning membranes on binary 6-MH/CPEO3 selfassembled monolayers . . . . . . . . . . . . . . . . . 46

$4.4 \quad$ Lateral membrane tension of pore-spanning membranes on 6-MH/CPEO3

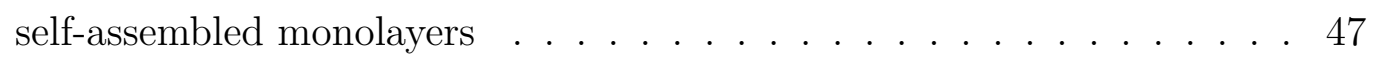

5 Discussion: Lateral membrane tension of pore-spanning membranes 53

5.1 Formation of binary 6-MH/CPEO3 self-assembled monolayer . . . . . . 53

$5.2 \quad$ Lateral membrane tension of pore-spanning membranes on 6-MH/CPEO3

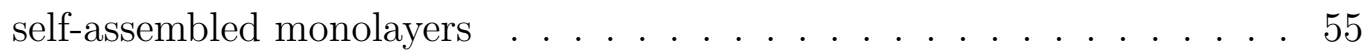

6 Results: SNARE-mediated fusion on pore-spanning membranes 59

6.1 Reconstitution of SNAREs into liposomes and pore-spanning membranes 60

6.2 SNARE-activity in liposomes $\ldots \ldots \ldots \ldots \ldots$

6.3 Mobility of lipids and SNAREs in pore-spanning membranes . . . . . . 65

6.4 SNARE-mediated single vesicle fusion assay . . . . . . . . . . 70

6.4 .1 Analysis of syb 2 vesicle size $\ldots \ldots \ldots \ldots$. . . . . . . 70

6.4 .2 Vesicle docking to pore-spanning membranes . . . . . . . . 71

$6.4 .3 \quad$ Mobility of docked vesicles $\ldots \ldots \ldots \ldots$. . . . . . . . . 73

$6.4 .4 \quad$ Fusion of vesicles with pore-spanning membranes $\ldots \ldots .78$

6.4 .5 Post fusion structures of vesicles . . . . . . . . . . . . . . . 82

$6.4 .6 \quad$ Intermediate TexasRed intensity levels of vesicles . . . . . . . 84

6.4 .7 Docking times of small and large vesicles . . . . . . . . . 87

7 Discussion: SNARE-mediated fusion on pore-spanning membranes 89

7.1 SNARE reconstitution in vesicles and pore-spanning membranes . . . . 89

7.2 Mobility of lipids and SNAREs in pore-spanning membranes . . . . . . 91

7.3 SNARE-mediated single vesicle fusion assay $\ldots \ldots \ldots \ldots . \ldots . . \ldots 94$

$7.3 .1 \quad$ Vesicle docking to pore-spanning membranes . . . . . . . . . . 94

7.3 .2 Interpretation of fusion events . . . . . . . . . . . . . . 99

7.3 .3 Post fusion structures of vesicles . . . . . . . . . . . . . 103 
7.3 .4 Kinetic analysis of fusion events . . . . . . . . . . . . . . . . . 104

\begin{tabular}{lll}
\hline 8 & Conclusion & 109
\end{tabular}

$\begin{array}{lll}9 & \text { Appendix } & 113\end{array}$

$9.1 \quad$ Amino acid sequences of SNAREs . . . . . . . . . . . . . . . . . . 113

$9.1 .1 \quad$ Amino acid sequence of synaptobrevin 2 . . . . . . . . . . 113

$9.1 .2 \quad$ Amino acid sequence of syntaxin 1A . . . . . . . . . . . . . . 113

9.1.3 Amino acid sequence of syntaxin 1A-transmembrane domain . . 113

9.1 .4 Amino acid sequence of SNAP 25a . . . . . . . . . . . . . . . 114

9.2 List of symbols and abbreviations . . . . . . . . . . . . . . . . . . 114

9.3 List of chemicals and consumables . . . . . . . . . . . . . . . . . 116

9.4 List of devices and software . . . . . . . . . . . . . . . . . 117

9.5 Software . . . . . . . . . . . . . . . . . . . . . . . . . . . . 119

9.6 Composition of expression media . . . . . . . . . . . . . . . . . . 119

9.7 Composition of SDS-PAGE buffer . . . . . . . . . . . . . . 120

9.8 Composition of SDS-PAGE gels . . . . . . . . . . . . . . . . . . . 121

9.9 Compositions of isolation buffers. . . . . . . . . . . . . . . . . 122

\begin{tabular}{lr}
\hline Bibliography & 125
\end{tabular} 



\section{Introduction}

Cell membranes of almost all living organisms consist of a lipid bilayer. The lipid bilayer resembles a several nanometer thick barrier protecting cells against their surrounding and subdividing organelles. Lipid bilayers are impermeable for most molecules preventing them from freely diffusing within the whole cell. To enable the controlled exchange of the molecules between the compartments, exocytosis and endocytosis enable an active transport mechanism, which expels or engulfs these molecules. During exocytosis, membrane-bound vesicles fuse with the cell membrane to secret their content into the extracellular environment. Endocytosis resembles the counterpart to exocytosis. A very important exocytotic process can be found in synapses. In synaptic boutons, fusion of synaptic vesicles with the presynaptic membrane leads to the release of neurotransmitters for stimulus conduction. Soluble N-ethylmaleimide-sensitive-factor attachment receptors (SNAREs) were identified as the driving force for this process, but in the last years also mechanical properties of the plasma membrane, like curvature and membrane tension, were identified as important regulators [1, 2]. To get a better understanding of the different processes and to elucidate the impact of curvature and tension, it is desirable to utilize artificial lipid membranes, which allow to study such processes in a well defined environment.

\subsection{Membrane tension as a regulator for exocytosis}

Cellular processes, like exocytosis, membrane trafficking or cell motility, are regulated by proteins. Many studies furthermore revealed, that also physical parameters of the membrane, like curvature and tension, drastically influence and control such processes [1-4]. The tension in the plasma membrane mainly arises from the adhesion between the membrane and the underlying cytoskeleton [1, 5]. In cell membranes the tension ranges between $0.003-0.45 \mathrm{mN} \mathrm{m}^{-1}$ and is highly variable depending on the cell type [6] 9]. Membrane area and membrane tension are generally closely linked, allowing the cells to control their tension by changes of the membrane area and cytoskeletal 
attachments. Therefore, membrane vesicle trafficking, e.g. exocytosis and endocytosis, are believed to be important processes, which regulate membrane area controlling cell shape and migration [4, 10]. During exocytosis, additional lipid material is fused in the cell membrane, increasing the membrane area and lowering the tension. Endocytosis on the other hand, decreases the membrane area in the plasma membrane as vesicular intermediates bud from the membrane and increase the membrane tension.

Experimental evidence for the interaction between membrane tension and exocytosis could be obtained by Gauthier et al. They showed that an artificial increase in membrane tension, generated by an hypotonic shock, activated an exocytotic burst in mouse embryonic fibroblasts [11]. These observations are supported by findings from Staykova et al., who coupled a lipid bilayer to an elastic sheet. They demonstrated that upon stretching of the elastic support, the bilayer expands without rupturing by fusing adhered vesicles giving rise to a passive area adaption of the membrane [12]. Mesoscopic simulations, based on dissipative particle dynamics also predict an optimal membrane tension for successful fusion of vesicles with a planar membrane. The fusion probability in the simulations also significantly raised, when the membrane tension was increased [13]. Yet, in the last two studies fusion was independent of proteins. It is however known that membrane fusion is a regulated process in cells driven by highly conserved proteins, like SNAREs. SNAREs are one of the most intensively studied fusion proteins and are essential during neurotransmitter release in synapses. Many insights in the mechanism of SNARE-mediated membrane fusion could be gained during the last years, but the exact mechanism is however still unclear [14]. It is therefore desirable, to develop in vitro model membrane systems for fusion with variable tensions to further investigate the impact of tension on membrane fusion.

\subsection{SNARE-mediated membrane fusion}

Membrane fusion describes the process by which the hydrophoarbic core of two initially separated lipid bilayers is merged, building a single interconnected structure. In eukaryotic cells, many processes involve membrane fusion, like the transport of lipids and proteins between the organelles, fertilization of an egg by sperm or neuronal fusion upon signal transduction in nerve terminals [15, 16]. In the context of this work neuronal fusion will be discussed in detail in the following. During signal transduction in nerve cells, neurotransmitters are released in the synaptic cleft via synaptic vesicles that fuse with the pre-synpatic membrane. This process is highly regulated and 
triggered by the influx of calcium ions. The overall time between the influx of calcium ions and the release of neurotransmitters requires less than one millisecond [17]. Many insights could be gained during the last years by analyzing synaptic vesicle fusion in cells [18, 19], but central mechanisms are still unclear making it desirable to analyze the fusion process in more detail.

Neuronal fusion is driven by SNAREs. SNARE is an acronym derived from the SNAP receptor. The existence of SNAREs was discovered in several consecutive studies showing that neuronal SNAREs resemble a 1:1:1 complex formed from the three proteins synaptobrevin 2, syntaxin 1A and SNAP 25 (synaptosome associated protein of $25 \mathrm{kDa}$ ) [20 23]. In 1998, Weber et al. experimentally verified that these proteins are sufficient to fuse membranes in vitro [24]. Synaptobrevin 2 is found in the membrane of synaptic vesicles, which is why it is also referred to as VAMP-2 (vesicle associated membrane protein-2). More often, synaptobrevin is called vesicularSNARE (v-SNARE). The protein is anchored in the vesicle membrane via an $\alpha$-helical transmembrane domain (TMD). Syntaxin 1A and SNAP 25 are located in the presynaptic membrane forming an active 1:1 complex to which synaptobrevin 2 can bind. Therefore, these proteins are also named target-SNAREs (t-SNAREs). Binding of synaptobrevin 2 to the syntaxin 1A/SNAP 25 complex results in the formation of a stable four helix bundle, called SNARE-core complex, which releases enough energy to initiate fusion between the vesicle and the pre-synaptic membrane. The SNARE-core complex consists of four parallel orientated $\alpha$-helices, one from synaptobrevin 2 , one from syntaxin 1A and two from SNAP 25, resembling a superhelical coiled-coil structure (Fig. 1.1] [15]. Formation of the coiled-coil structure is realized by the highly conserved SNARE-motif consisting of a repeating pattern of amino acids, called heptad repeat [25, 26]. The heptad repeat consists of two hydrophobic amino acids at position 1 and 4 , while the other positions are occupied by polar and hydrophilic amino acids. The hydrophobic residues interact with each other in the coiled-coil structure stabilizing it.

Studies of Pobbati et al. showed that binding of synaptobrevin 2 to the t-SNARE complex takes place from the $\mathrm{N}$ - to the C-terminus in a zipper-like fashion, bringing the membranes close together [28]. Figure 1.2 schematically depicts different states of SNARE-mediated membrane fusion. Zippering of v-SNAREs (red) and the t-SNAREs (blue) brings the distinct membranes close together. A rhombohedral fusion stalk [29, 30] is formed due to rearrangements of the lipids in the adjacent membrane leaflets leading to a hemifusion diaphragm [31 33]. The geometry of the fusion stalk resembles a highly curved structure, requiring the lipids to be bend in this region. Therefore 


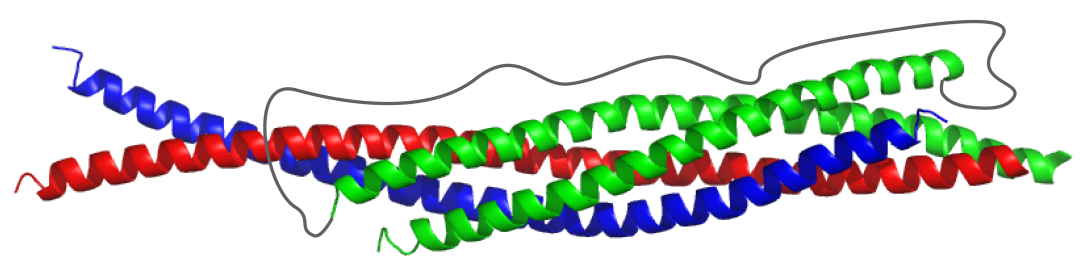

Figure 1.1. Ribbon plot of the synaptic cis-SNARE complex consisting of the SNARE motifs of syntaxin 1 (red), synaptobrevin-2 (blue) and SNAP-25 (green) derived from $\mathrm{X}$-ray diffraction [27].

lipids with a packing parameter that supports the formation of negative curvature, are beneficial for fusion. Lipids resembling a geometry, that favors a positive curvature like lysophophatidylcholine were found to inhibit the formation of a fusion stalk [34, 35]. The process of fusion pore opening is not fully understood and it remains controversial if and how SNAREs catalyze the transition from a stalk to the fusion pore [36, 37].

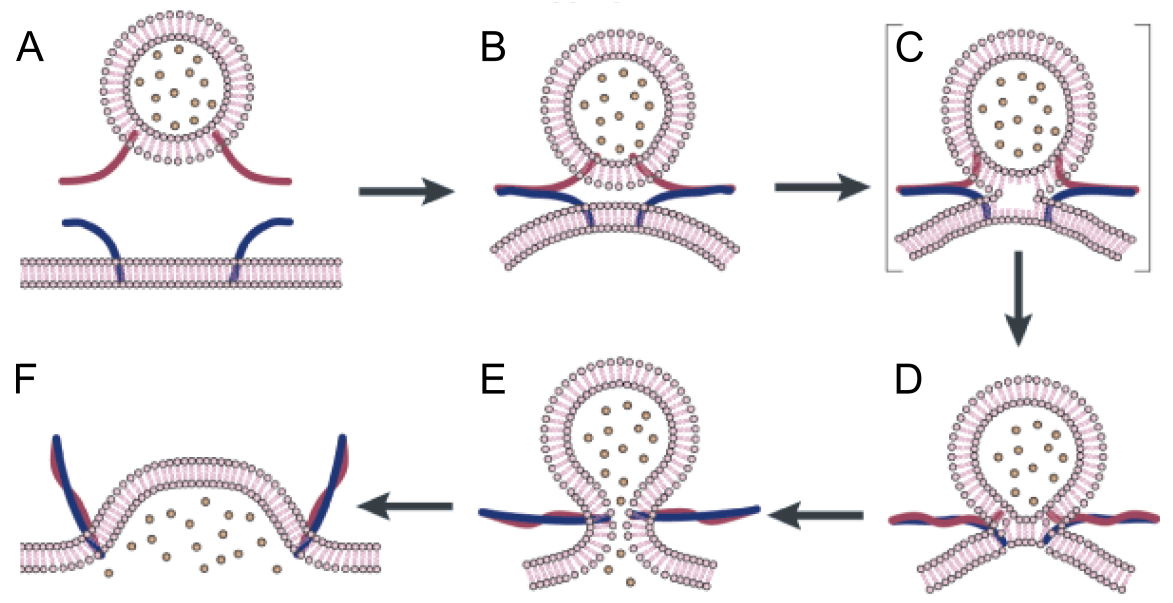

Figure 1.2. Model of SNARE-mediated membrane fusion derived from Chen et al. [38. (A) Membranes are close to each other but SNAREs do not interact. (B) SNAREs interact and start to zipper from the $\mathrm{N}$ - to the C-terminus drawing the membranes together. (C) Due to the close contact of the membranes and the increasing curvature, hemifusion occurs. (D) The distal membrane leaflets come into contact to avoid the unfavorable space at the membrane junction, building a hemifusion diaphragm. (E) The hemifusion structure breaks down, resulting in a fusion pore. (F) The fusion expands allowing the membrane to relax. 


\subsection{In vitro assays for membrane fusion}

Several different in-vitro membrane systems were developed to investigate membrane fusion in a well defined environment and under controlled conditions in the past decades. In vitro systems allow experimental manipulations and observations, which are not possible in neuronal cultures. To set up an artificial membrane fusion system, synaptic proteins are expressed in suitable hosts (e.g. E. coli), extracted, purified and reconstituted into lipid bilayers composed of either synthetic lipids or lipids obtained from cell extracts. The most popular fusion assays, which can be generally divided into ensemble and single-vesicle experiments are discussed in the following. An outstanding critical review about the different fusion assays was published recently by Brunger et al. 39 .

\subsubsection{Ensemble vesicle fusion assays}

\section{Vesicle-vesicle assays}

Ensemble vesicle-vesicle fusion assays are commonly used for the analysis of lipid and content mixing. Weber et al. were the first who used a fluorescence based vesicle-vesicle fusion assay, initially developed by Struck et al. in 1981, to demonstrate that SNAREs are fusogens [24, 40]. In their assay, they utilized two vesicle populations with one population carrying the v-SNARE synaptobrevin and the other population carrying the t-SNAREs syntaxin and SNAP 25 (Fig. 1.3). One vesicle population was additionally labeled with the lipid-anchored fluorophores NBD-PE and Rh-PE. Due to the close proximity of two fluorophores in the vesicles a Förster Resonance Energy Transfer (FRET) between the donor NBD-PE and the acceptor Rh-PE resulted in a quenching of NBD-PE. When the vesicle populations were mixed and fused, lipid mixing between the labeled and unlabeled vesicles lead to the dilution of the fluorophores resulting in a dequenching of NBD-PE. This assay was modified by Schwenen et al., by labeling the v-SNARE vesicles with acceptor fluorophores and the t-SNARE containing vesicles with donor fluorophores. Upon lipid mixing, a FRET between the fluorophores resulted in an intensity increase of the acceptor fluorophores [41].

A major disadvantage of the ensemble lipid mixing assays is that one can not distinguish between different fusion states like hemifusion or full fusion since lipid mixing alone does not reliably indicate content mixing, which can only occur upon full fusion. To overcome these disadvantages and also differentiate between full fusion and hemifusion, vesicle-vesicle assays were developed in which the outer fluorophores (those pointing 


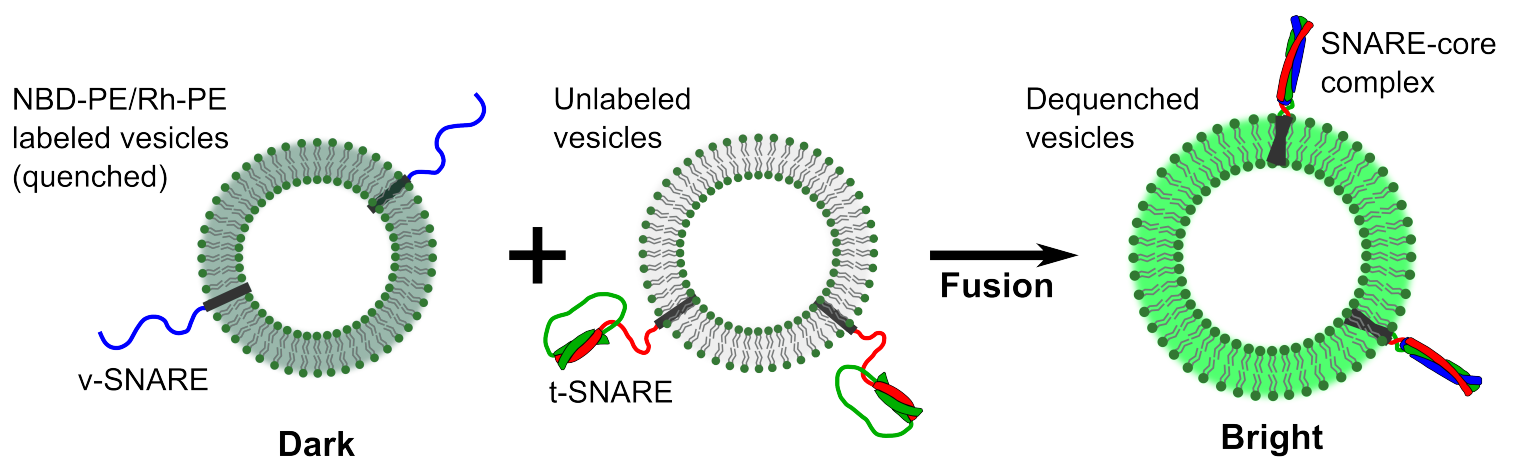

Figure 1.3. Ensemble fusion assay of vesicles containing SNAREs used by Weber et al. [24]. v-SNARE vesicles containing NBD-PE and Rh-PE were mixed with unlabeled t-SNARE vesicles. Upon fusion and lipid mixing, NBD-PE and Rh-PE are diluted resulting in a dequenching of NBD-PE.

out of the vesicles) are reduced by the agent dithionate. This way, only the inner fluorophores contribute to the dequenching signal indicating inner leaflet mixing and therefore full fusion [32]. Although this method displays a powerful and nice way to analyze fusion intermediates, it suffers from the fact that outer and inner leaflet mixing can occur without content mixing due to flip-flop and lipid-dye transfer from adjacent membranes 42 44].

\subsubsection{Single vesicle fusion assays}

\section{Vesicle-vesicle assays}

Single vesicle-vesicle fusion assays were developed to circumvent the disadvantages of ensemble fusion assays. One major approach to achieve this is to tether one vesicle population on a surface and add the second population to the immobilized vesicles. Yoon et al. developed a system in which they tethered t-SNARE containing vesicles labeled with the acceptor fluorophore DiD via biotin-neutravidin interaction to a biotin-polyethyleneglycol coated glass surface and added v-SNARE containing vesicles labeled with the donor fluorophor DiI to the immobilized vesicles [45]. Lipid mixing was subsequently observed by total internal reflection (TIRF) microscopy considering the FRET efficiencies. With this approach they could distinguish between docked and fused vesicles. However, the assay did not allow to distinguish between hemifusion and full fusion.

In order to discriminate between hemifusion and full fusion, a single vesicle-vesicle content mixing assay was developed using an indicator for content- and lipid-mixing, 
simultaneously [46, 47]. This assay allowed to distinguish between a variety of different fusion events including hemifusion, fast content mixing and delayed content mixing.

\section{Vesicle-planar bilayer assays}

Although single vesicle-vesicle assays allow to investigate lipid and content mixing simultaneously to obtain information about fusion intermediates, they suffer from the fact that the target-membrane is highly curved and not flat as observed in synaptic boutons. To consider this aspect but to maintain the possibility of single vesicle fusion detection, assays utilizing a supported planar target membrane to which freely diffusing vesicles are added were developed by several different groups [48 50].

Bowen et al. published an assay using calcein filled mobile vesicles containing dyelabeled synaptobrevin, which were added to a planar glass supported membrane containing syntaxin (Fig. 1.4) [48]. By simultaneously monitoring the content and membrane fluorescence, they were able to discriminate between docking and fusion of the vesicles with the planar membrane. However, SNARE fusion was rare, e.g. independent of SNAP 25, and apparently triggered by laser light. Wang et al., who used a similar assay, mainly detected vesicle bursting rather than controlled fusion with the target-membrane [51]. A similar approach was established by Fix et al. also relying on glass supported target-membranes (Fig. 1.4] [49]. In contrast to the assay published by Bowen, they used the fluorescently labeled lipids NBD-PE and Rh-PE, which resemble a FRET pair also initially used in the first ensemble vesicle-vesicle assays (see Chapter 1.3.1). They could monitor docking, undocking as well as fusion. Diffusion of the fluorophores in the planar membrane indicated lipid mixing.

Besides the rather inconsistent finding of these assays, most of the studies showed SNAP 25 independent fusion, indicating that fusion is most probably not triggered by SNAREs. A possible explanation for this might be the limited mobility of the proteins in the planar membrane due to the direct condensation of small liposomes on the glass surface [39].

To overcome the rather poor mobilities in the glass supported membranes, planar membranes were prepared on polyethylene glycol (PEG) brushes on glass, often referred to as polymer-cushions, to decouple the membrane from the support [52, 53]. In one of the approaches, small vesicles with reconstituted t-SNAREs were directly condensed on the PEG coated glass surface to obtain a supported bilayer [52]. In the second approach, a lipid monolayer was first formed on the PEG-support by a LangmuirBlodgett transfer technique to which small t-SNARE containing vesicles were added. 


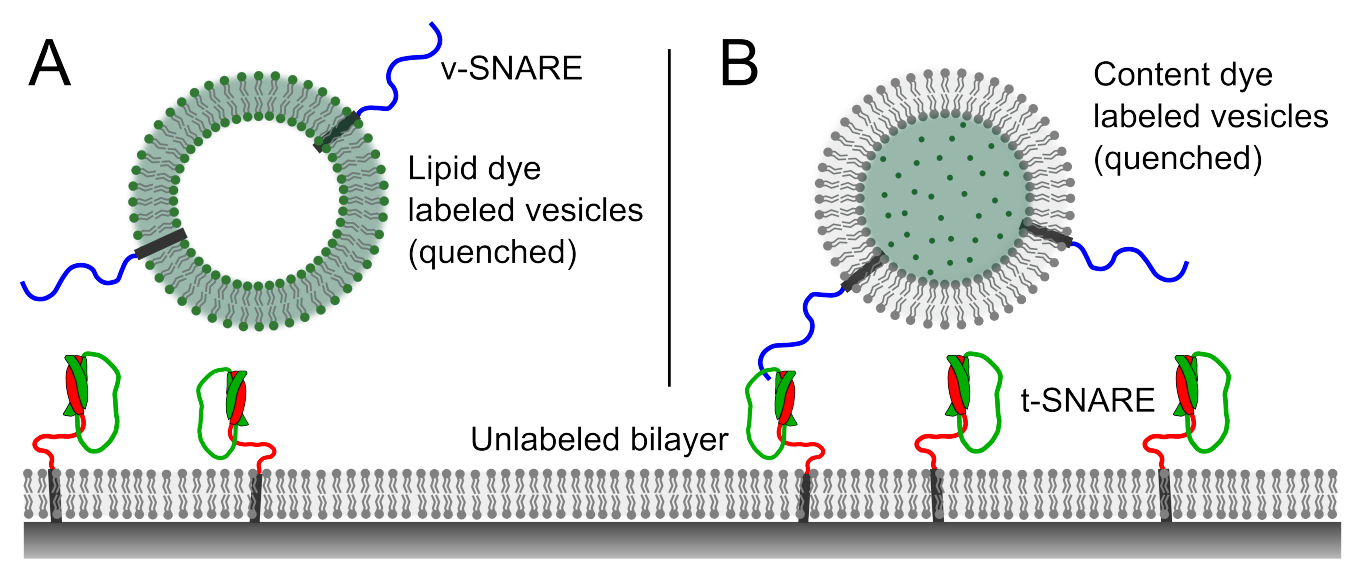

Figure 1.4. Single-vesicle bilayer fusion assay. (A) Fusion assay developed by Fix et al. [49]. using lipid labeled vesicles, which fuse with an unlabeled lipid bilayer formed on a glass surface. (B) Fusion assay published by Bowen et al. [48] using content labeled vesicles, which fuse with an unlabeled lipid bilayer. In both assays, fusion was monitored by time dependent fluorescence microscopy. Lipid mixing was indicated by a sudden increase in fluorescence intensity at the bilayer surface subsequently followed by a decay as the molecules diffuse away. Reconstituted proteins are synaptobrevin 2 (blue), syntaxin 1A (red) and SNAP 25 (green).

The vesicles fused with the monolayer, resulting in an entire lipid bilayer [53]. Both assays showed SNAP 25 dependent fusion, indicating that fusion in these system is driven by SNAREs. A combination of a lipid and a content mixing indicator was also recently used in these systems to discriminate not only between docking and fusion but also between hemifusion and full fusion [54].

Inspired by the various fusion assays based on entirely supported planar targetmembranes, Höfer and Schwenen et al. recently developed planar pore-spanning membranes (PSMs) as a suitable system to investigate SNARE-mediated membrane fusion [55, 56]. PSMs are composed of a lipid bilayer patch which is spanned over a functionalized porous microsieve structure harboring cylindrical holes resulting in supported membranes on the pore rim and freestanding membranes spanning the pores (Fig. 1.5). The areas between the pores, called pore rims were functionalized with a hydrophilic self-assembled monolayer (SAM) to decouple the membrane from the support. The PSMs contained the t-SNARE $\Delta$ N-complex (see Chapter 3.1.4) and were fluorescently labeled with OregonGreen-DPPE (OG). Large unilamellar vesicles reconstituted with the v-SNARE synaptobrevin 2 and fluorescently labeled with TexasRed-DPPE (TR) were added to the PSMs and docking as well as fusion was monitored with an upright confocal laser scanning microscope. For the experiments, 
OG and TR were excited with a single laser line $(488 \mathrm{~nm})$ and separately detected. This allowed to analyze docking and undocking of vesicles, as well as lipid mixing due to FRET between the donor OG and the acceptor TR on supported and freestanding membranes.

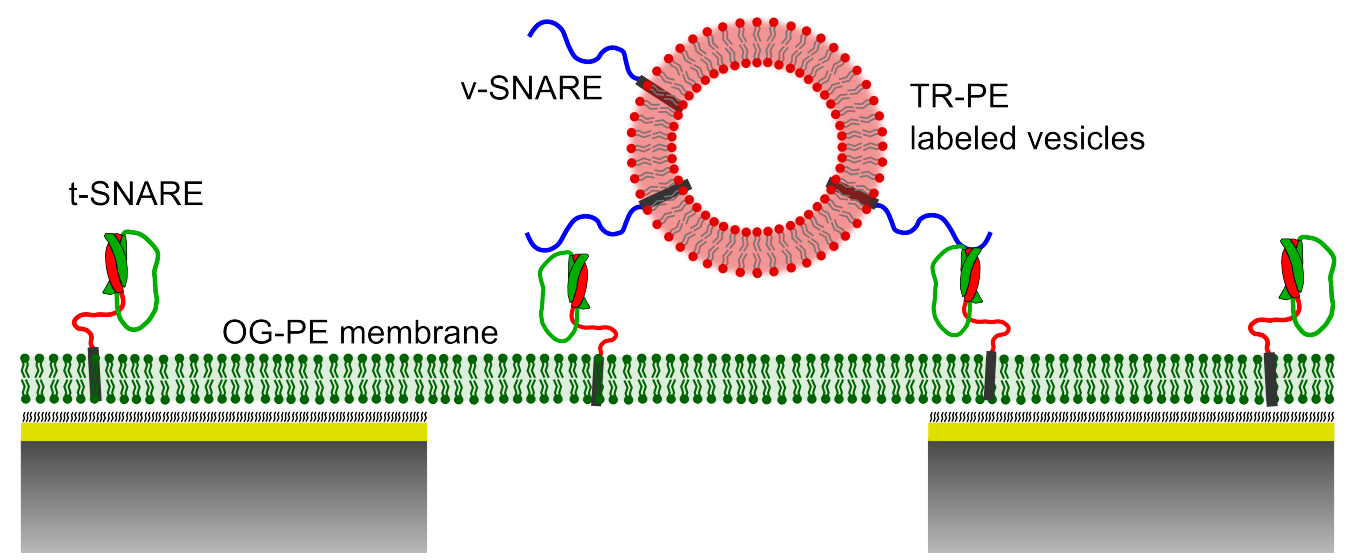

Figure 1.5. Single-vesicle PSM fusion assay. Fusion of TR labeled vesicles containing the v-SNARE synaptobrevin 2 with an OG labeled PSM containing the t-SNARE $\Delta \mathrm{N}$ complex is monitored by CLSM. Lipid mixing was observable by a FRET between the donor OG and the acceptor TR.

\subsubsection{SNAREs in in vitro fusion assays}

SNARE-mediated fusion requires a 1:1 complex of syntaxin 1A/SNAP 25, which assembles in an ordered fashion with synaptobrevin 2 [57, 58]. In plasma membranes, SNAREs are clustered in nanodomains and assemble in a sequential reaction rather than random collision [18, 59]. In order to study SNARE-mediated fusion in in-vitro assays, syntaxin and SNAP 25 are often co-reconstituted into target-membranes [48, 50]. This direct reconstitution resembles a straight forward way but it suffers from the fact, that syntaxin $1 \mathrm{~A}$ and SNAP 25 easily form a stable 2:1 four helix bundle in the membrane, where the binding site of synaptobrevin 2 is occupied by a second syntaxin $1 \mathrm{~A}$. The 2:1 complex itself resembles an inactive, so called "dead end" complex, which is not able to catalyze fusion anymore (Fig. 1.6] [28]. To prevent the formation of inactive t-SNARE complexes, Pobbati et al. developed a stabilized t-SNARE acceptor complex ( $\Delta \mathrm{N}$-complex) composed of syntaxin $1 \mathrm{~A}$, SNAP 25 and a small synaptobrevin fragment (aa 49-96). The synaptobrevin 2 fragment occupies part of the synaptobrevin binding site, preventing binding of a second syntaxin 1A, but leaving the N-terminal 
part free. This allows the full length v-SNARE synaptobrevin 2 to bind to the Nterminal end and displace the small fragment upon SNARE core-complex formation. The four helix bundle of the core-complex is sufficient to drive fusion between lipid bilayers [28, 32, 53]. Fluorescence anisotropy measurements however revealed that the displacement of the synaptobrevin 2 fragment takes several seconds, slowing down the initial SNARE-complex formation [28].

Kreutzberger et al. recently reported a new strategy to assemble membrane-bound 1:1 syntaxin 1A/SNAP 25 complexes without a stabilizing synaptobrevin 2 fragment [60]. They isolated syntaxin 1 in its monomeric form using the detergent dodecylphosphocholine (DPC), which was subsequently assembled with SNAP 25. The obtained 1:1 SNARE-complex also showed high fusogenity, but in a direct comparison with the $\Delta \mathrm{N}$-complex, no improvement of the fusion rates could be observed.

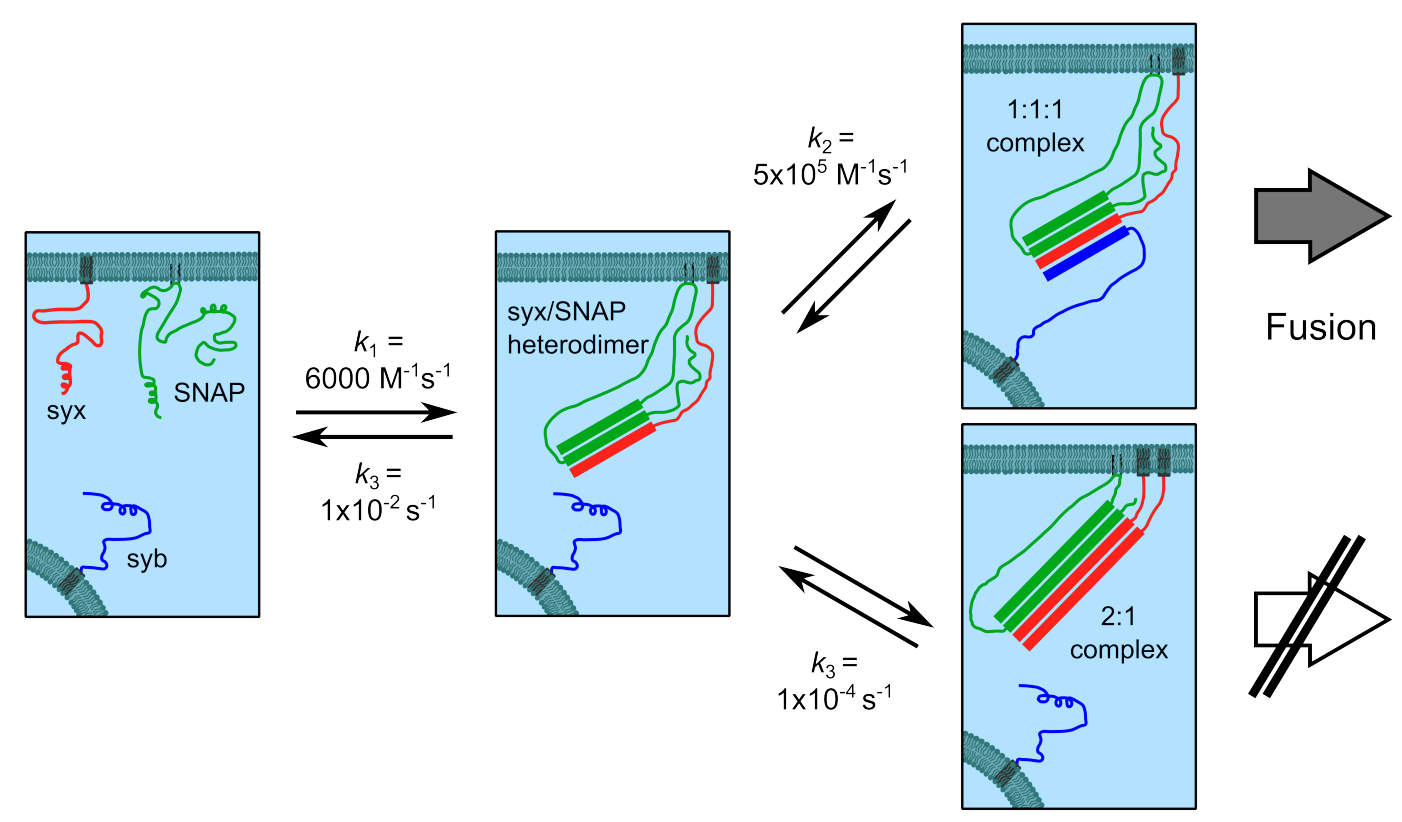

Figure 1.6. Kinetic model of the different SNARE assembly pathways modified from Pobbati et al. [28]. The rate constants were determined by soluble portions of the SNAREs rather than reconstituted in two fusing membranes [58. In the first step syntaxin 1A (syx) and SNAP 25 (SNAP) assemble into a 1:1 complex. The complex provides a binding site for either synaptobrevin (syb) or syx. Binding of syx leads to a stable 1:1:1 complex, which is off-pathway as syb can not displace syx anymore. Binding of syb leads to a 1:1:1 SNARE-core complex, which initiates fusion between the membranes. 


\section{Scope}

Upon stimulus conduction in nerve terminals, synaptic vesicles fuse with the presynaptic membrane and release neurotransmitters in the synaptic cleft. This complex process of membrane fusion is highly regulated and driven by soluble N-ethylmaleimide-sensitivefactor attachment receptors (SNAREs) and physical properties of the membrane such as tension and curvature stress. The exact mechanism of SNARE-mediated fusion is however unclear and the impact of membrane tension on this process remains poorly understood.

Pore-spanning membranes (PSMs), consisting of supported and partly freestanding lipid bilayers prepared on a porous microchip, were recently used as a versatile membrane model system to monitor fusion on a single vesicle level. The freestanding membranes of the PSMs enable to analyze fusion without the influence of an underlying support. Additionally, a significant lateral membrane tension in the freestanding membranes, arising from the preferred adhesion of the membrane on the support around the pores, allows to study fusion in a tension dependent manner. The aim of this thesis is to establish PSMs as a membrane model system in which the lateral membrane tension can be actively modulated to monitor single SNARE-mediated fusion events with high temporal resolution by fluorescence microscopy.

PSMs are prepared on different binary self-assembled monolayers. The lateral membrane tension of the PSMs will then be investigated as a function of the composition of the monolayer. With this strategy it is explored how and if membrane tension in PSMs can be modulated by chemical surface modifications. SNARE-mediated membrane fusion will then be analyzed to investigate the influence of lateral membrane tension on fusion. Additionally, the impact of membrane curvature on the fusion kinetics will be analyzed in this system using small and large vesicles for fusion. A focus will be drawn on dynamic aspects of vesicle docking and fusion on the supported and freestanding membranes to elucidate the impact of the solid support on the fusion process, which is not addressable in many other artificial fusion systems. 



\section{Materials and Methods}

\subsection{Materials}

\subsubsection{Porous substrates}

Porous substrates used to prepare pore-spannning membranes were manufactured by a photolithographic process in silicon, a common technique used in the semiconductor production (fluXXion B.V., Eindhoven, The Netherlands and Aquamrijn B.V., Zutphen, The Netherlands). The substrates had a size of $5 \times 5 \mathrm{~mm}$ and provided a stable, $1 \mathrm{~mm}$ wide frame supporting the $800 \mathrm{~nm}$ thick porous part in the center. Substrates with pore diameters of $1.2 \mu \mathrm{m}$ (Figure 3.1A) and $5 \mu \mathrm{m}$ (Figure $3.1 \mathrm{~B}$ ) with a hexagonal pore arrangement and a surface porosity of $35-40 \%$ were used in this work.
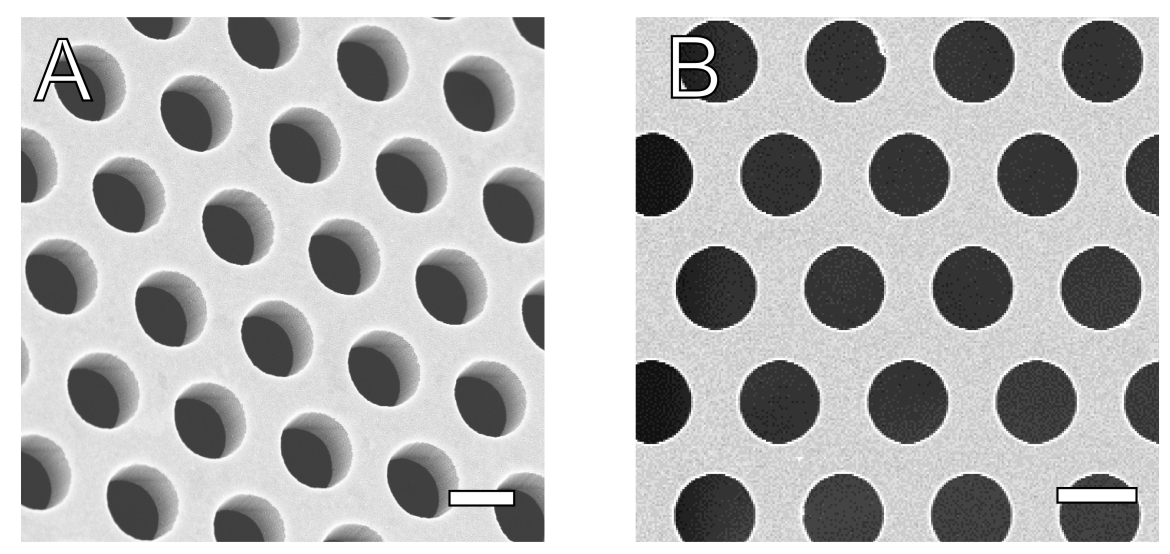

Figure 3.1. Scanning electron micrographs of porous substrates used in this work. (A) Substrate with a hexagonal arrangement of the pores with diameters of $1.2 \mu \mathrm{m}$. Scale bar: $1 \mu \mathrm{m}$. (B) Substrate with pores with diameter of $5 \mu \mathrm{m}$ hexagonally arranged. Scale bar: $5 \mu \mathrm{m}$. 


\subsubsection{Lipids}

Pore-spanning membranes composed of different lipids were used in this work. For the analysis of the lateral membrane tension, the synthetic lipid 1,2-diphytanoyl-snglycero-3-phosphocholine (DPhPC) was used to prepare the membranes (Fig. 3.2 A). $\mathrm{DPhPC}$ does not exhibit a detectable gel to liquid crystalline phase transition over a wide temperature range $\left(-120-120^{\circ} \mathrm{C}\right)$ and has a large area compression modulus $K_{a}$ of $670 \mathrm{mN} / \mathrm{m}$ which is significantly higher than those of naturally occurring lipids [61]. This makes DPhPC membranes very stable and is the reason why it is frequently used in in-vitro membrane model systems 62 64. For the fusion experiments a lipid mixture composed of 1,2-Dioleoyl-sn-glycero-3-phosphocholine (DOPC), 1-Palmitoyl2-oleoyl-sn-glycero-3-phosphoethanolamin (POPE), 1- Palmitoyl-2-oleoyl-sn-glycero-3phospho-L-serin (POPS) and cholesterol (Fig. 3.2B-E) in a molar ratio of 5:1:2:2 was used to mimic the lipid composition of synaptic vesicles [65].

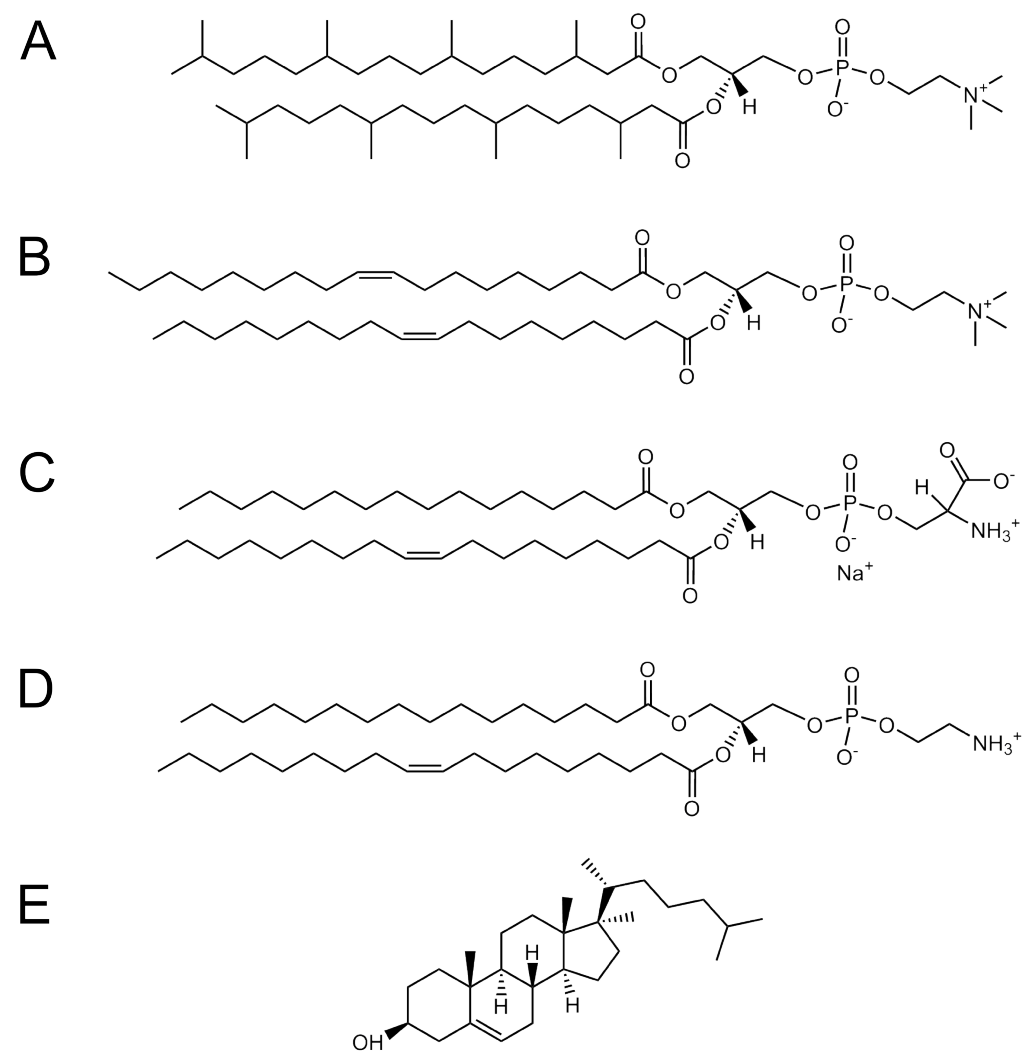

Figure 3.2. Structural formulas of the lipids used in this work. (A) DPhPC, $\mathrm{C}_{48} \mathrm{H}_{96} \mathrm{NO}_{8} \mathrm{P}, M_{r}=846.3 \mathrm{~g} / \mathrm{mol}$. (B) DOPC, $\mathrm{C}_{44} \mathrm{H}_{84} \mathrm{NO}_{8} \mathrm{P}, M_{r}=786.1 \mathrm{~g} / \mathrm{mol}$. (C) POPS, $\mathrm{C}_{40} \mathrm{H}_{75} \mathrm{NO}_{10} \mathrm{PNa}, M_{r}=784.0 \mathrm{~g} / \mathrm{mol}$. (D) $\mathrm{POPE}, \mathrm{C}_{39} \mathrm{H}_{76} \mathrm{NO}_{8} \mathrm{P}, M_{r}=$ $718.0 \mathrm{~g} / \mathrm{mol}$. (E) cholesterol, $\mathrm{C}_{27} \mathrm{H}_{46} \mathrm{O}, M_{r}=386.7 \mathrm{~g} / \mathrm{mol}$. 


\subsubsection{Fluorescence probes}

Fluorescence microscopy was used to control the formation of PSMs and to investigate SNARE-mediated fusion. Therefore all membranes were either doped with 1 mol\% of the lipid coupled fluorescent dye TexasRed-DPPE (TR-DPPE) with an absorption maximum $\lambda_{\mathrm{abs}}=595 \mathrm{~nm}$ and an emission maximum at $\lambda_{\mathrm{em}}=615 \mathrm{~nm}$ or Atto488DPPE with $\lambda_{\text {abs }}=500 \mathrm{~nm}$ and $\lambda_{\text {abs }}=520 \mathrm{~nm}$ (Figure 3.3A and B). Both fluorophores are very photostable and have high extinction coefficients which make them ideal for high speed fluorescence microscopy experiments. Atto488 is also capable of transferring energy to TR via nonradiative dipole-dipole coupling when the fluorophores are in close contact. This process is called Förster Resonance Energy Transfer (FRET) and is inversely proportional to the sixth power of the distance between the donor (Atto488) and acceptor (TR) which makes this technique extremely sensitive to changes in the distance and therefore suitable to analyze lipid mixing.

A

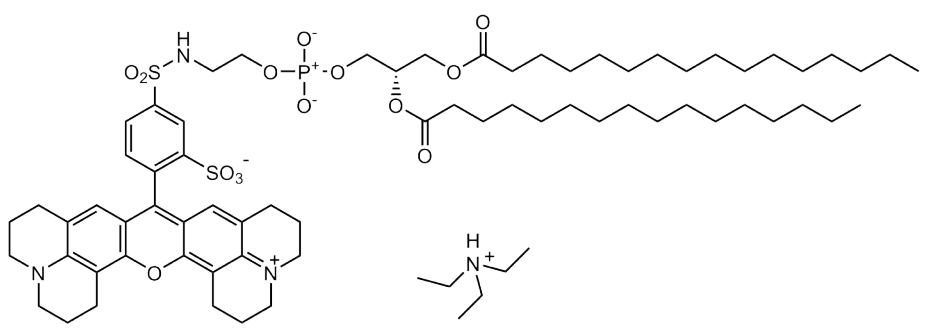

B

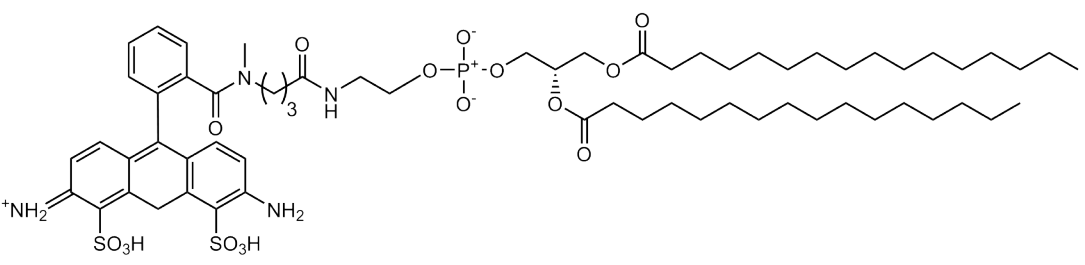

Figure 3.3. Structural formulas of the TexasRed and Atto488 labeled lipid 1,2dipalmitoyl-sn-glycero-3-phosphoethanolamine (DPPE). (A) TexasRed-DPPE triethylammoniumsalt, $M_{\mathrm{r}}=1381.8 \mathrm{~g} / \mathrm{mol}$. (B) Atto488-DPPE, $M_{\mathrm{r}}=1263.2 \mathrm{~g} / \mathrm{mol}$.

\subsubsection{SNAREs}

SNAREs used for the fusion experiments were vesicular-SNARE (v-SNARE) synaptobrevin 2 (residues 1-116) and a modified target-SNARE (t-SNARE) acceptor complex called $\Delta \mathrm{N}$-complex developed by Pobbati et al. [28]. The $\Delta \mathrm{N}$-complex was assembled from the proteins syntaxin $1 \mathrm{~A}$ (residues 183-288) lacking the regulatory $\mathrm{H}_{\mathrm{abc}}$ domain, 
SNAP 25a (residues 1-206) and a small water soluble fragment of synaptobrevin 2 (residues 49-96). The syb 2 fragment binds partially to the SNARE-motif of syx 1A and SNAP 25a preventing the complex from binding a second syx 1A which would result in an inactive 2:1 complex. Upon fusion, full length syb 2 binds to the unoccupied $N$-terminal part of the SNARE-motif and displaces the syb 2 fragment from the $\Delta \mathrm{N}$-complex forming a tight four helical SNARE-bundle, which initiates fusion (Figure 3.4). For the control experiments, a syb 2 fragment (residues 1-96) comprising the full SNARE-motif but lacking the $C$-terminal transmembrane domain was used to irreversible block the $\Delta \mathrm{N}$-complex to specifically inhibit fusion.

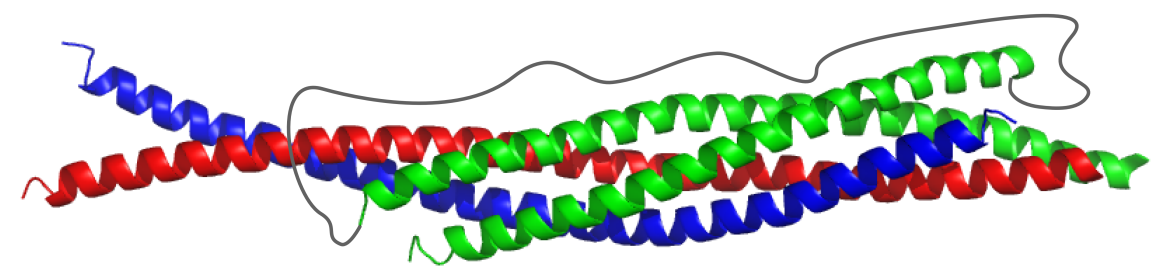

Figure 3.4. Ribbon plot of the synaptic SNARE complex assembled from Syntaxin1A (red), Synaptobrevin-2 (blue) and SNAPE-25a (green) derived from X-ray diffraction [27]. The four helix bundle is highly stable and SDS resistant.

\section{Synaptobrevin 2}

Synaptobrevin $2(\operatorname{syb} 2,12.691 \mathrm{kDa}, \mathrm{pI}=7.84, \epsilon=13980)$ consists of an cytosolic part (residues 1-94) carrying the SNARE-motif (residues 31-91) and a transmembrane domain (residues 95-114) that anchors the protein in the membrane (Fig. 3.5). Syb 2 can bind to the $\Delta \mathrm{N}$-complex forming a tetrameric coiled-coil structure called SNARE-core complex. Due to the hydrophobic transmembrane domain, syb 2 can only be handled in solutions that contain detergents like 3-[(3-cholaminidopropyl)dimethylammonio]1 propanesulfonate (CHAPS). Syb 2 has a mainly $\alpha$-helical secondary structure. The water soluble synaptobrevin fragment (residues 49-96, 5.627 kDa, pI =9.16, $\epsilon=12490$ ) was used to assemble the stabilized $\Delta \mathrm{N}$-complex. A syb 2 fragment with the full SNARE motif (residues 1-96, $10.517 \mathrm{kDa}, \mathrm{pI}=8.04, \epsilon=12660$ ) was used for the control experiments. 


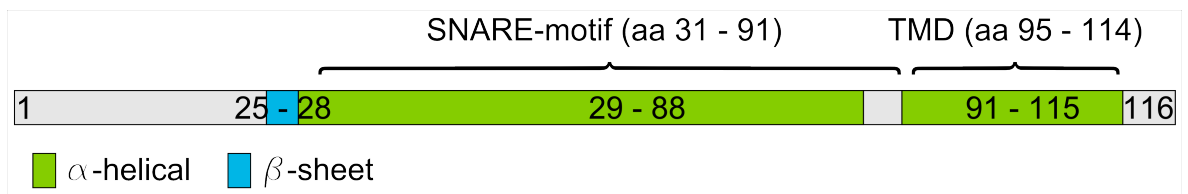

Figure 3.5. Schematic drawing of the secondary structure of synaptobrevin 2 (amino acids 1-116). The grey parts resemble unstructured protein segments [27].

\section{Syntaxin 1A}

Syntaxin 1A (12.035 kDa, $\mathrm{pI}=5.90, \epsilon=4470)$ was used in a shortened variant (residues 183-288) lacking a large part of the cytosolic part that has regulatory functions presenting mainly the SNARE motif (residues 192 - 254) and the transmembrane domain (residues 266 - 288) that anchors the protein in the target-membrane. The secondary structure of syx $1 \mathrm{~A}$ is schematically shown in Figure 3.6 .

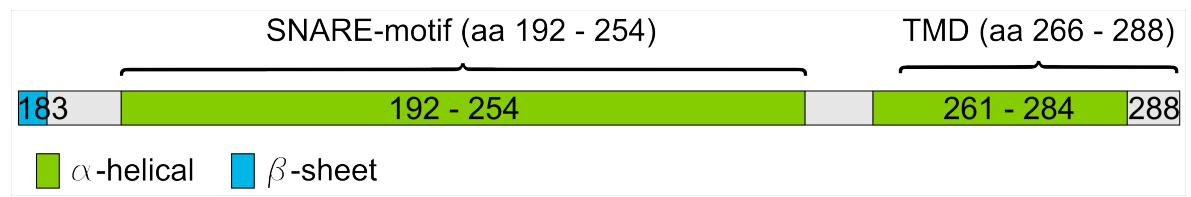

Figure 3.6. Schematic drawing of the secondary structure of syntaxin $1 \mathrm{~A}$ (residues 183-288). Grey parts resemble unstructured protein segments [27].

\section{SNAP 25a}

SNAP 25 was used with its full sequence (residues 1-206) but in a mutated variant (SNAP 25a) with all four cysteine replaced by alanine $(23.207 \mathrm{kDa}, \mathrm{pI}=4.74, \epsilon=$ 7240). The cysteines (positions 85, 88, 90 and 92), which anchor SNAP 25 in the pre-synaptic membrane via palmitoyl linker, were replaced to exclude site reactions during the expression in E. coli as no post translational modifications take place at these positions. SNAP 25a consists of two $\alpha$-helical parts (residues 7 - 82 and residues 142 - 201) that harbor two SNARE motifs (Fig. 3.7) which are involved in the assembly of the tetrameric coiled-coil complex.

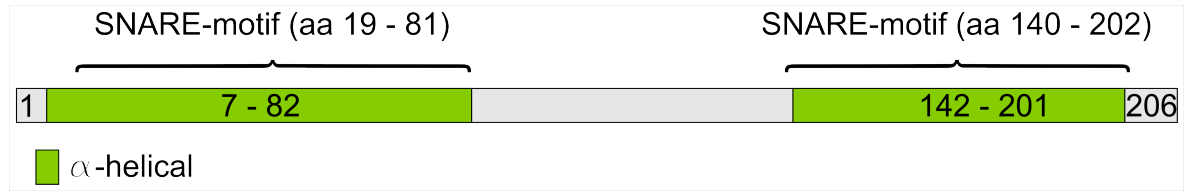

Figure 3.7. Schematic drawing of the secondary structure of SNAP 25a (residues 1-206). Unstructured segments of the protein is marked by the grey parts [27]. 


\section{$\Delta \mathrm{N}$-complex}

The $\Delta$ N-complex (40.885 kDa, $\epsilon=24200)$ was assembled from SNAP 25a, syx 1A and syb 2 (residues 49-96) in a 1:1:1 ratio prior to reconstitution into liposomes (Fig. 3.8). The $\Delta \mathrm{N}$-complex is stable in solution but denatures in sodium dodecyl sulfat (SDS) 28.

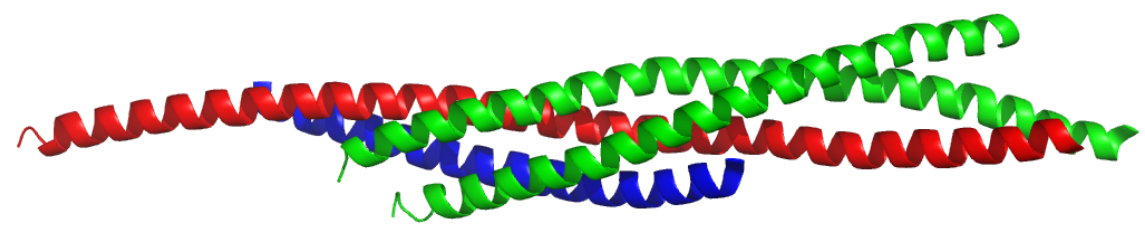

Figure 3.8. Ribbon plot of the assembled $\Delta$ N-complex [28]. SNAP 25a is shown in green, syx $1 \mathrm{~A}$ in red and syb 2 (residues 49-96) in blue.

\subsection{Preparative methods}

\subsubsection{Giant unilamellar vesicles}

Giant unilamellar vesicles used for the preparation of PSMs that were probed by atomic force microscopy, were prepared by mixing the lipids (DPhPC,TR-DPPE, 99:1) dissolved in chloroform in a test tube and applying the solution onto two indium tin oxide (ITO) covered glass slides. Evaporation of the chloroform resulted in the formation of a lipid film. Any remaining chloroform was removed in vacuum for 30 minutes. The ITO slides were then assembled together in a way that the sides with the lipid films faced each other (Fig. 3.9p. A sealing between the two slides allowed to fill the space between the slides with sucrose solution $(200 \mathrm{~mm})$. The ITO surfaces were each connected via copper stripes with one pole of a sinus generator. A sinusoidal wave was applied for 3 hours (1.6 V(peak-peak), $10 \mathrm{~Hz}$ ) resulting in the formation of GUVs. The GUVs were then harvested carefully from the the chamber in fractions of $400 \mu$ l. Each fraction was analyzed by fluorescence microscopy to choose the fraction with the highest amounts of GUVs. 


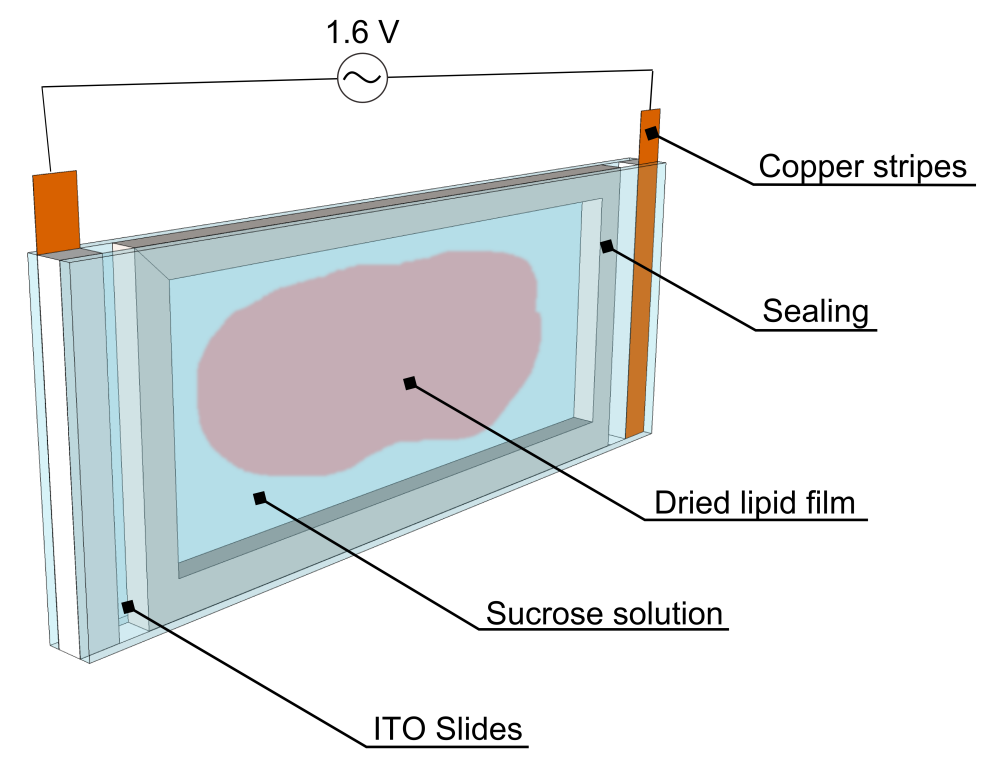

Figure 3.9. Schematic drawing of an assembled electroformation chamber. A sinusoidal voltage of $1.6 \mathrm{~V}$ (peak-peak) with a frequency of $10 \mathrm{~Hz}$ was applied on each copper electrode for $3 \mathrm{~h}$.

\subsubsection{Isolation and purification of SNAREs}

SNAREs, originating from rattus norvegicus, were heterologously expressed in E. coli (BL21 (DE3)) containing a pET28 vector. Each protein was provided with a hexahistidineTag (His-6-tag) for the purification via immobilized metal ion affinity chromatography that was cut of by a thrombin digest after isolation. Final purification and concentration was performed by ion exchange chromatography. The isolation and purification procedure is broadly similar for all SNARE proteins and therefore described in general in the following. Deviating conditions are annotated individually for each protein.

Glycerol stocks of transformed E. coli cells, carrying the vector that encodes for the individual SNARE proteins were used for expression. The glycerol stocks were kindly provided by the Jahn group from the Max Planck Institute for Biophysical Chemistry in Göttingen. Several microliter of each glycerol stock were used to inoculate LB medium (50 ml, $30 \mu \mathrm{g} / \mathrm{ml}$ Kanamycin) at $37^{\circ} \mathrm{C}$ over night as a preculture. The main culture was then prepared by inoculating 10x250 ml TB medium (30 $\mu$ g Kanamycin) with each $5 \mathrm{ml}$ preculture and incubating at $37^{\circ} \mathrm{C}$ until an optical density of 0.9 was reached. Expression was induced with $0.25 \mathrm{mM}$ IPTG for $2.5 \mathrm{~h}$ at $37^{\circ} \mathrm{C}$. The cell suspension was centrifuged at $4000 \mathrm{rpm}$ for $20 \mathrm{~min}$, the supernatant discarded, the pellets resuspended in $80 \mathrm{ml}$ extraction buffer and stored at $-20{ }^{\circ} \mathrm{C}$. The frozen, resuspended pellets were defrosted at room temperature and $10 \mathrm{mg}$ lysozyme, $1 \mathrm{mM}$ 
$\mathrm{MgCl}_{2}, 2 \mathrm{mg}$ DNAse I and one tablet of cOmplete (EDTA-free) protease inhibitor was added and the suspension stirred for $20 \mathrm{~min}$. After homogenization with a teflon potter, $80 \mathrm{ml}$ extraction buffer were added and the suspension passed two times through a microfluidizer (Microfluidizer LM10, Microfluidics Corp., MA, USA) at 1000 bar. The isolation of syx $1 \mathrm{~A}$ required the adjustment of the suspension to $6 \mathrm{M}$ urea prior to centrifugatio for $45 \mathrm{~min}$ at $13500 \mathrm{rpm}$ at $4{ }^{\circ} \mathrm{C}$. The supernatant was mixed with $8 \mathrm{ml} \mathrm{Ni}{ }^{2+}$-Agarose and incubated at $4{ }^{\circ} \mathrm{C}$ for $2 \mathrm{~h}$ on a roller mixer. The slurry was filtered through a BioRad Econo-column and washed with $250 \mathrm{ml}$ washing buffer. Syb 2 was additionally washed in a second step with washing buffer II. The proteins were subsequently eluted with elution buffer $(3 \times 10 \mathrm{ml})$, combined and supplemented with $10 \mathrm{mM}$ dithiothreitol (DTT). Thrombin $(2 \mathrm{mg})$ was added and the solutions dialyzed against dialysis buffer over night at $4{ }^{\circ} \mathrm{C}$. Syx $1 \mathrm{~A}$ required a second dialysis step in dialysis buffer II over night. Dialysis tubings with a molecular weight cutoff (MWCO) of 4-6 kDa were used for all proteins. The cleavage of the His-6-tag was checked by SDS-PAGE in a 10\% Schaegger gel. All proteins were further purified on a Äkta ion exchange system with either MonoQ or MonoS columns (GE Healthcare, Little Chalfont, UK) using Äkta buffer A and B to remove thrombin and increase the final protein concentration. The collected Äkta fractions were finally analyzed by SDS-PAGE to combine the fractions with the highest purity and yield. Ion exchange chromatograms and corresponding SDS-PAGE gels from the elution fractions of syx 1A, syb 2, SNAP 25a and the $\Delta \mathrm{N}$-complex are shown in the following. The proteins syb 2 (residues 49-96) and syb 2 (residues 1-96) were isolated by the former co-worker Lando Schwenen. The compositions of the buffer solutions, expression media and SDS-PAGE gels are listed in the Appendix (Chapter 9).

\section{Synaptobrevin 2}

Synaptobrevin 2 was purified on a strong cation exchange column (MonoS 5/50, GE Healthcare, Little Chalfont, UK). The resulting elution chromatogram shows a single UV absorption peak (Fig. 3.10A) eluting at a buffer B concentration of $40 \%$. The indicated elution fractions 9-16 were further analyzed by SDS-PAGE (Fig. 3.10B). The dominant Coomassie stained band at $13 \mathrm{kDa}$ can be assigned to synaptobrevin 2 $(12.691 \mathrm{kDa})$ indicating successful isolation. Fractions $11-15$ were combined and the total protein concentration determined by UV-vis spectroscopy (Nanodrop2000, Thermo Scientific, MA, USA). A total of $6.2 \mathrm{mg}$ synaptobrevin 2 were isolated from 2.51 culture. 
A

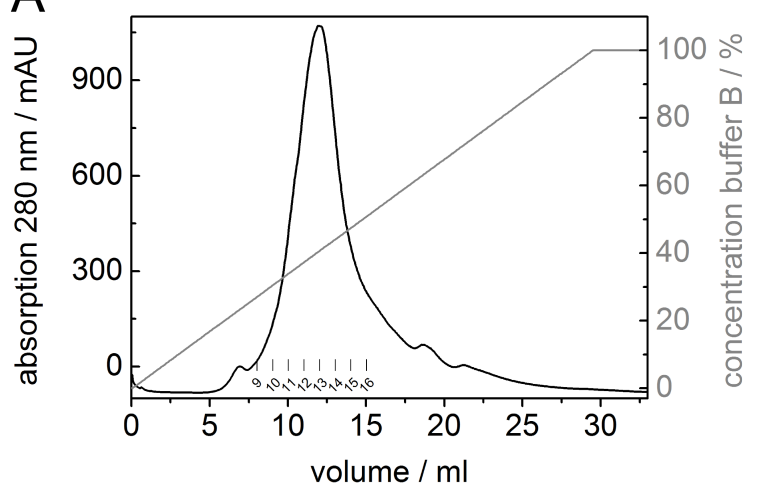

B

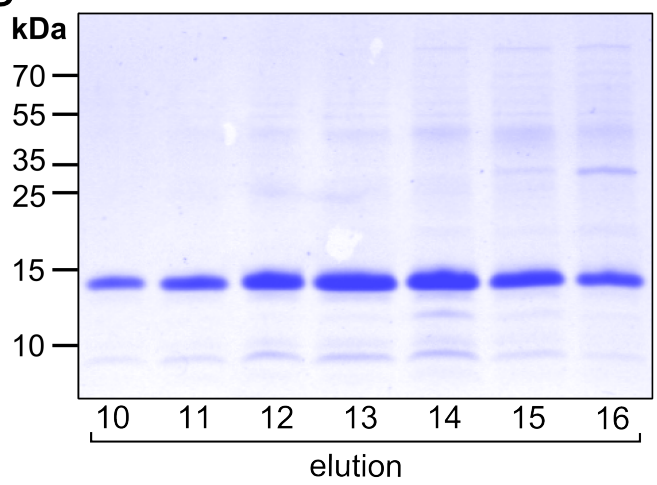

Figure 3.10. (A) Ion exchange chromatogram observed after purification of syb 2 on a MonoS 5/50 column. Syb 2 elutes at a buffer B concentration of 40\%. The fractions 9-16 were collected and analyzed by SDS-PAGE showing a dominant band at $13 \mathrm{kDa}(\mathrm{B})$.

\section{Syntaxin 1A}

Syntaxin 1A was purified on a strong anion exchange column (MonoQ 10/100, GE Healthcare, Little Chalfont, UK). The resulting elution chromatogram shows a single absorption peak (Fig. 3.11A) eluting at a buffer B concentration of $30 \%$. The indicated elutions 9-14 were further analyzed by SDS-PAGE (Fig. 3.11B). The Coomassie stained band at $12 \mathrm{kDa}$ can be assigned to syntaxin $1 \mathrm{~A}(12.035 \mathrm{kDa})$ showing successful isolation. Fractions 9-13 were combined and the total protein concentration determined by UV-vis spectroscopy. A total of $63 \mathrm{mg}$ syntaxin $1 \mathrm{~A}$ were isolated from $2.5 \mathrm{l}$ culture.
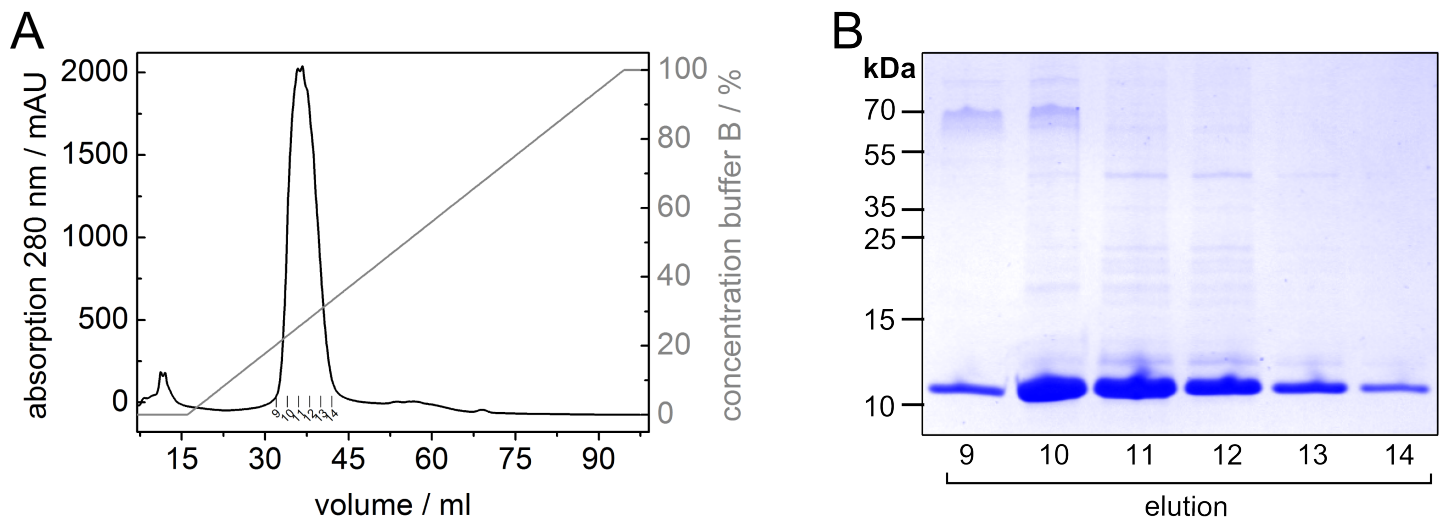

Figure 3.11. (A) Ion exchange chromatogram observed after purification of syx $1 \mathrm{~A}$ on a MonoQ 10/100 column. Syx $1 \mathrm{~A}$ elutes at a buffer B concentration of $30 \%$. The fractions 9-13 were collected and analyzed by SDS-PAGE showing a dominant band at $12 \mathrm{kDa}$ originating from syx $1 \mathrm{~A}(\mathrm{~B})$. 


\section{SNAP 25a}

SNAP 25a was purified on a strong anion exchange column (MonoQ 10/100, GE Healthcare, Little Chalfont, UK). The resulting elution chromatogram shows several absorption peaks (Fig. 3.12A) eluting at buffer B concentrations of 10\%, $18 \%$ and $50 \%$ (A). Fractions $39-45$ of the peak at $18 \%$ buffer B were analyzed by SDS-PAGE showing a dominant band at $23 \mathrm{kDa}$ and a minor band at $11 \mathrm{kDa}$ (Fig. $3.12 \mathrm{~B}$ ). The band at $23 \mathrm{kDa}$ could be assigned to SNAP 25a (23.207 kDa) indicating successful isolation of the target protein. Fractions 40-45 were combined and the total protein concentration determined by UV-vis spectroscopy. A total of $5 \mathrm{mg}$ SNAP 25a were isolated from 2.51 culture.
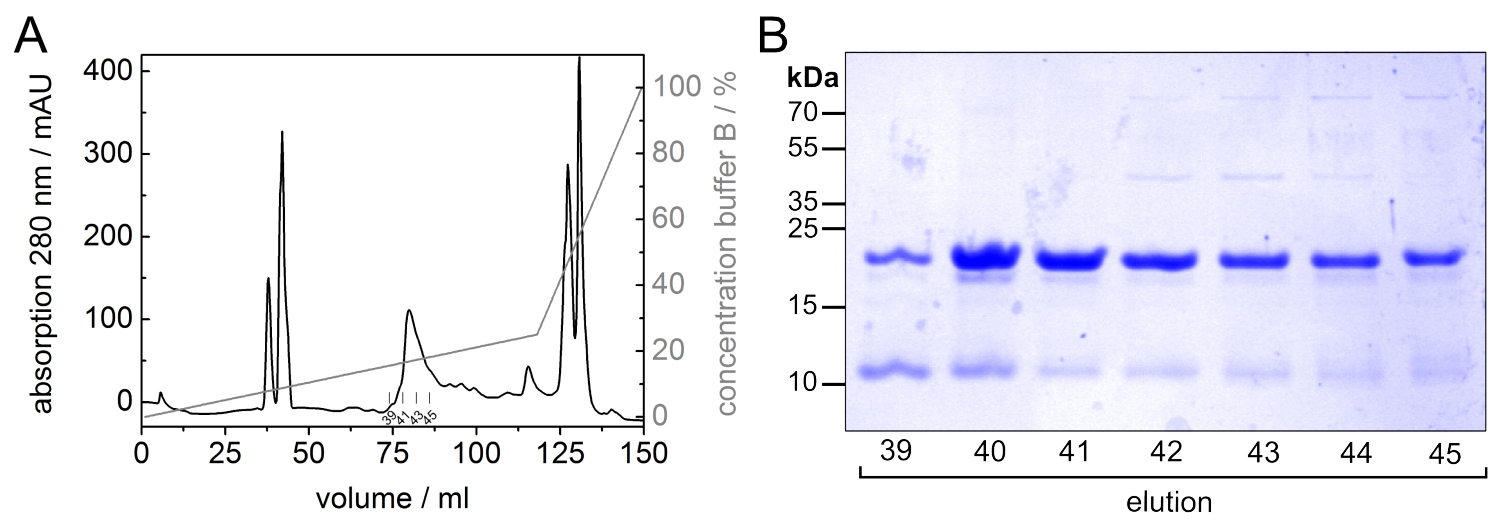

Figure 3.12. (A) Ion exchange chromatogram observed after purification of SNAP 25a on a MonoQ 10/100 column. SNAP 25a elutes at a buffer B concentration of $20 \%$. The fractions 40-45 were collected and analyzed by SDS-PAGE showing a dominant band at $23 \mathrm{kDa}(\mathrm{B})$.

\section{Syb 2 C79 (residues 49-96)}

Syb 2 C79 (residues 49-96) is a mutant of the water soluble syb 2 fragment (residues 49-96) with the serine replaced by cysteine at position 79. This allows to fluorescently label the fragment with maleimide coupled dyes. Syb 2 C79 (residues 49-96) was purified on a strong cation exchange column (MonoS 5/50). The elution chromatogram shows a single absorption peak (Fig. 3.13A) eluting at a buffer B concentration of $50 \%$. Fractions 15-20 were analyzed by SDS-PAGE showing a dominant band with a molecular weight smaller than $10 \mathrm{kDa}$, which can be assigned to syb $2 \mathrm{C} 79$ (residues 49-96) (5.64 kDa) indicating successful isolation of the target protein (Fig. 3.13B). Fractions 15-20 were combined and the total protein concentration determined by 
UV-vis spectroscopy. A total of $2 \mathrm{mg}$ syb 2 C79 (residues 49-96) were isolated from 2.5 l culture.
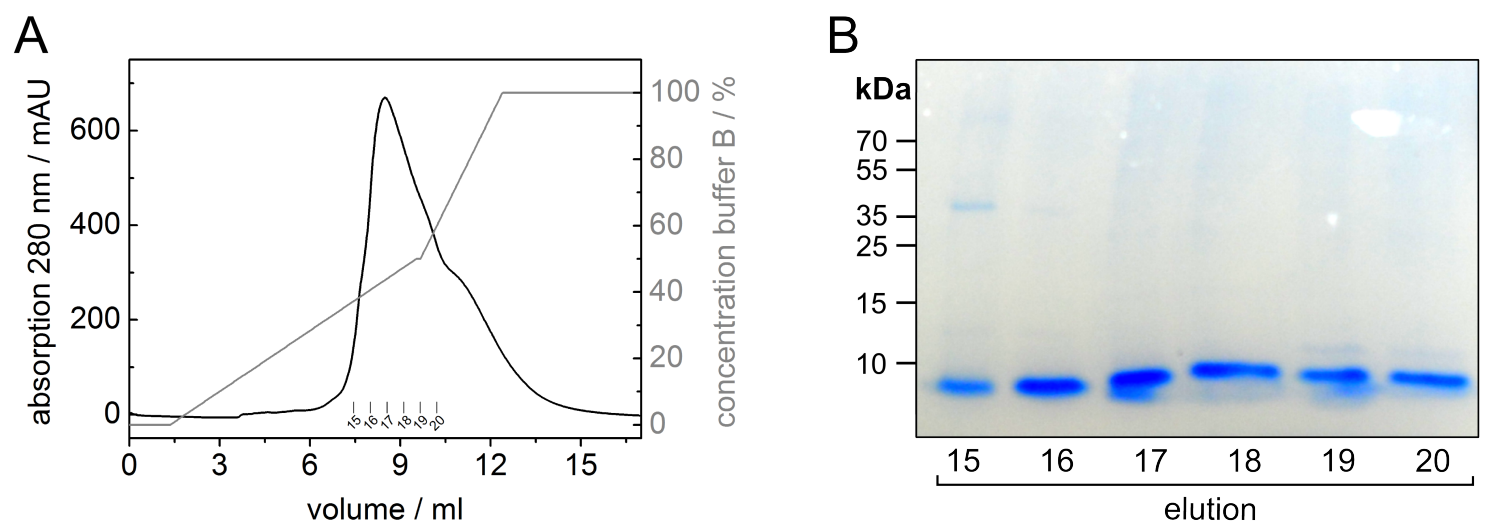

Figure 3.13. (A) Ion exchange chromatogram observed after purification of syb 2 C79 (resdiues 49-96) on a MonoS 5/50 column. syb 2 C79 (resdiues 49-96) elutes at a buffer B concentration of 40-50\%. Fractions 15-20 were collected and analyzed by SDS-PAGE showing a dominant band at $<10 \mathrm{kDa}(\mathrm{B})$.

\section{$\Delta \mathrm{N}$-complex}

The $\Delta \mathrm{N}$-complex was assembled from the proteins SNAP 25a, syx 1A and syb 2 (amino acids 49-96). Prior to assembly, the SNAP 25a and syb 2 (amino acids 49-96) fractions were supplemented with 1\% CHAPS. Syx 1A and syb 2 (amino acids 49-96) were pre-mixed at a molar ratio of (1:2) for 30 minutes. SNAP 25a was then added, so that the final mixture had a molar ratio of 1:2:2 (syx 1A : sy 2 (aa 49-96) : SNAP 25a). The mixture was incubated over night at $4{ }^{\circ} \mathrm{C}$ and then purified by ion exchange chromatography on a MonoQ 5/50 column. Figure 3.14A shows the elution chromatogram of the $\Delta \mathrm{N}$-49-complex. A single peak eluting at $8 \mathrm{ml}$ (70\% buffer B) can be observed. The excess of syb 2 (amino acids 49-96) and SNAP 25a can not be observed as they elute well before the $\Delta \mathrm{N}$-complex and were therefor washed from the column with a constant buffer mixture ( $40 \%$ buffer B) to increase the purity of the complex upon gradient elution. Fractions 10-12 were collected and analyzed by SDS-PAGE as shown in Figure 3.14B. Three dominant bands can be observed correlating to molecular weights of 23, 11 and over $10 \mathrm{kDa}$ from the proteins SNAP 25a, syx $1 \mathrm{~A}$ and syb 2 (amino acids 49-96) proof that the complex assembly was successful. 
A

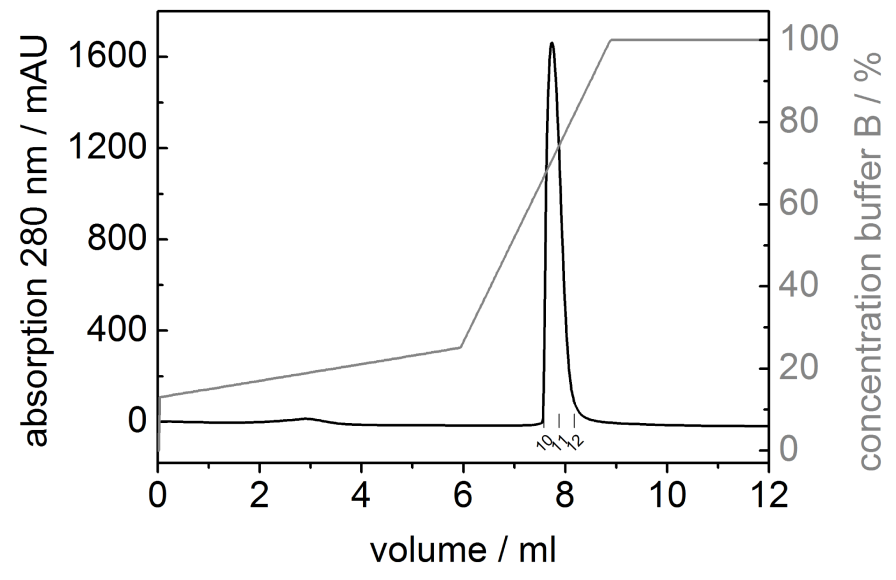

B

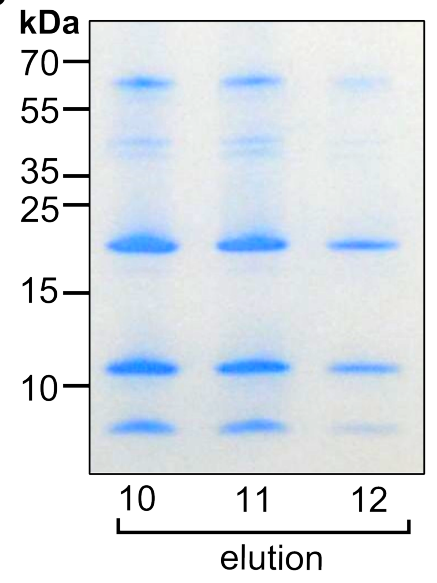

Figure 3.14. (A) Ion exchange chromatogram observed after purification of the $\Delta \mathrm{N}$ complex on a MonoQ 5/50 column. The $\Delta \mathrm{N}$-complex elutes at a buffer B concentration of $70 \%$. The fractions 10-12 were collected and analyzed by SDS-PAGE showing a dominant bands at 23,11 and over $10 \mathrm{kDa}(\mathrm{B})$.

\section{Fluorescent labeling of $\Delta \mathrm{N}$-complex}

The syb 2 C79 (residues 49-96) fragment was fluorescently labeled with Atto488maleimide (ATTO-TEC GmbH, Siegen, Germany) and assembled with syx 1A and SNAP 25a to obtain a fluorescently labeled $\Delta$ N-complex. The syb 2 fragment was labeled by dissolving $0.5 \mathrm{mg}$ Atto488-maleimide in $50 \mu \mathrm{l}$ Äkta buffer A and transferring it to $1 \mathrm{ml}$ syb 2 C79 (residues 49-96) solution $(0.4 \mathrm{mg} / \mathrm{ml}$ ), which was stirred for $2 \mathrm{~h}$ at room temperature. The reaction mixture was purified by size exclusion chromatography with a Sephadex G-15 column (diameter $=1 \mathrm{~cm}$, length $=30 \mathrm{~cm}$ ). The purified fractions were analyzed by UV/vis spectroscopy (Nanodrop2000, Thermo Scientific) to determine the degree of labeling (DOL) (Fig. 3.15A). The DOL was calculated from the absorbance of the protein at $280 \mathrm{~nm}\left(A_{\mathrm{syb}}\right)$ and from the absorbance of the dye at $505 \mathrm{~nm}\left(A_{505}\right)$ according to:

$$
\mathrm{DOL}=\frac{A_{505} \cdot \epsilon_{\mathrm{syb}}}{A_{\mathrm{syb}} \cdot \epsilon_{\mathrm{Atto} 488}}
$$

with $\epsilon_{\mathrm{syb} 2}=12490$ the extinction coefficient of syb 2 C79 (residues 49-96) and $\epsilon_{\text {Atto488 }}=$ 90000 the extinction coefficient of Atto488. Atto488 also partly absorbs at $280 \mathrm{~nm}$, therefore the absorption had to be corrected for the contribution of the dye with a factor of $\left.C F_{\text {Atto488 }}=0.09\right)$ to determine the absorption of the protein $A_{\text {syb }}$ itself: 


$$
A_{\mathrm{syb}}=A_{280}-\left(A_{505} \cdot C F_{\mathrm{Atto} 488}\right)
$$

After labeling and purification, $0.48 \mathrm{mg}$ Atto488-syb 2C79 (residues 49-96) were obtained with a DOL of $82 \%$. The labeled syb 2 fragment was assembled with syx $1 \mathrm{~A}$ and SNAP 25a and purified according to Chapter 3.2 .2 to obtain the Atto488- $\Delta \mathrm{N}$-complex. The UV-vis analysis of the labeled complex is shown in Figure $3.15 \mathrm{~B}$ yielding a DOL of $40 \%$.
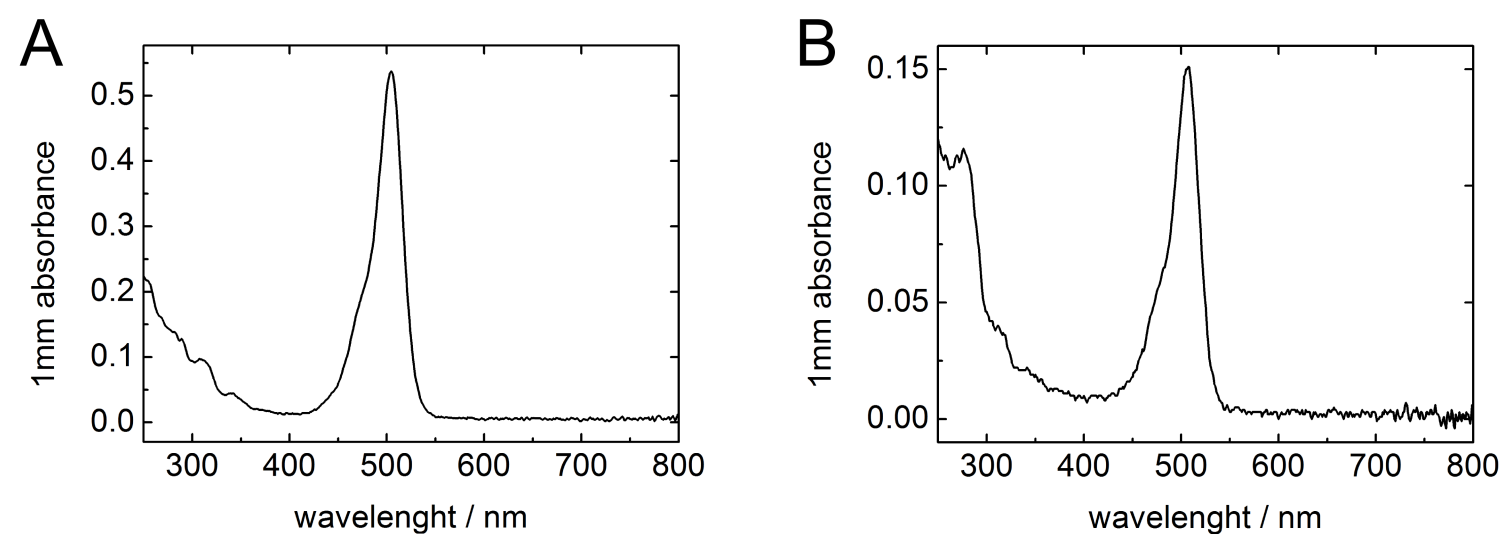

Figure 3.15. UV-vis spectra of Atto488-syb 2 C79 (residues 49-96) (A) and Atto488$\Delta \mathrm{N}$-complex (B).

\subsubsection{Reconstitution of SNAREs into liposomes}

SNARE containing vesicles were prepared from lipid films with a total mass of $0.5 \mathrm{mg}$ by mixing the lipids (DOPC, POPS, POPE, cholesterol, 5:2:1:2) dissolved in chloroform in a test tube following the protocol developed by the former co-worker Lando Schwenen [41]. The chloroform was subsequently evaporated by purging with nitrogen and heating to $30{ }^{\circ} \mathrm{C}$. To remove any remaining chloroform, the lipid films were dried in vacuum for 30 minutes. The lipid films were solubilized with $50 \mu$ l reconstitution buffer (20 mm HEPES, $100 \mathrm{~mm} \mathrm{KCl,} 1 \mathrm{~mm}$ EDTA, pH 7.4) containing $n$-Octyl $\beta$ D-thioglucopyranoside $(n$-OG). Protein solutions were added to the dissolved lipid films to a total $n$-OG concentration of $75 \mathrm{~mm}$ and a final protein to lipid (p:l) ratio of 1:500. The solutions were incubated for $30 \mathrm{~min}$ at room temperature and the detergent was removed via size exclusion chromatography (illustra NAP-25 G25 column, GE Healthcare, Little Chalfont, UK) in reconstitution buffer. A subsequent second size exclusion step was performed in MilliQ to remove remaining detergent and salt. This 
preparation yields small unilamellar $\Delta \mathrm{N}$-complex and syb 2 vesicles (proteo-SUVs). The proteo-SUVs serve as the basis for the preparation of large and giant unilamellar proteo-liposomes (proteo-LUVs and proteo-GUVs). To obtain proteo-LUVs the proteoSUV solution was dried in a glass cylinder over night in a desiccator over saturated sodium chloride solution with a relative humidity of $76 \%$. The slow drying process, supported by the high humidity, resulted in the formation of a proteo-lipid film that was re-hydrated by adding $500 \mu \mathrm{l}$ reconstitution buffer for 15 minutes. Mixing of the hydrated lipid film yielded multilamellar proteo-vesicles that were transformed into unilamellar proteo-LUVs by extrusion through polycarbonate membranes with a mini extruder (LiposoFast-Basic, Avestin) for 31 times. The size of the proteo-LUVs was controlled by using polycarbonate membranes with different nominal pore diameter of $50 \mathrm{~nm}$ and $400 \mathrm{~nm}$. Proteo-GUVs were prepared by drying proteo-SUV droplets $(2 \mu \mathrm{l})$ on ITO covered glass slides in a desiccator over saturated sodium chloride solution over night. The slow drying process resulted in the formation of a proteo lipid film on the ITO slides. The slides were then assembled together and subjected to electroformation as described for the GUV formation in Chapter 3.2.1 to obtain proteo-GUVs.

\subsubsection{Bulk SNARE activity experiments}

The activity of SNAREs in liposomes was tested by bulk experiments in a spectrofluorimeter (FP-6500, Jasco, Groß-Umstadt, Germany). $\Delta$ N-complex vesicles doped with $1 \mathrm{~mol} \%$ Atto488-DPPE $(150 \mu \mathrm{l})$ and syb 2 vesicles doped with $1 \mathrm{~mol} \%$ TR-DPPE $(150 \mu \mathrm{l})$ were mixed with $600 \mu \mathrm{l}$ reconstitution buffer in a cuvette and stirred at room temperature. The mixture was excited with light of the wavelenght $488 \mathrm{~nm}$ while perpendicular to the excitation beam the fluorescence emission at $608 \mathrm{~nm}$ was analyzed for $3000 \mathrm{~s}$. Upon docking and fusion of $\Delta \mathrm{N}$-complex and syb 2 vesicles Atto488 and TR come close together resulting in an förster resonance energy transfer (FRET) between the donor Atto488 and the acceptor TR resulting in an increase in TR fluorescence intensity which is measured.

\subsubsection{Pore-spanning membranes (PSMs)}

Porous substrates were cleaned with $n$-propanol and dried under a stream of nitrogen. The surface was then sputter coated (Cressington Sputter Coater 108auto) with titanium for $15 \mathrm{~s}$ (40 mA, 0.4 mbar) and subsequently coated by thermal evaporation with an orthogonal 30-40 nm thick gold layer as shown in Figure 3.16A. The orthogonal 
gold layer, sitting only on top of the substrate and not in the pore walls, is essential for the proper formation of PSMs. The gold coated substrates were then immersed into $1 \mathrm{mM} n$-propanolic solutions of 6-mercapto-1-hexanole 6-MH and cholesterylpolyethylenoxythiol CPEO3 (Fig. 3.16B/C) over night to form a densely packed self-assembled monolayer (SAM). For the force indentation experiments, binary 6-MH/CPOE3 SAMs were assembled, while for the single vesicle fusion experiments pure 6-MH SAMs were prepared.
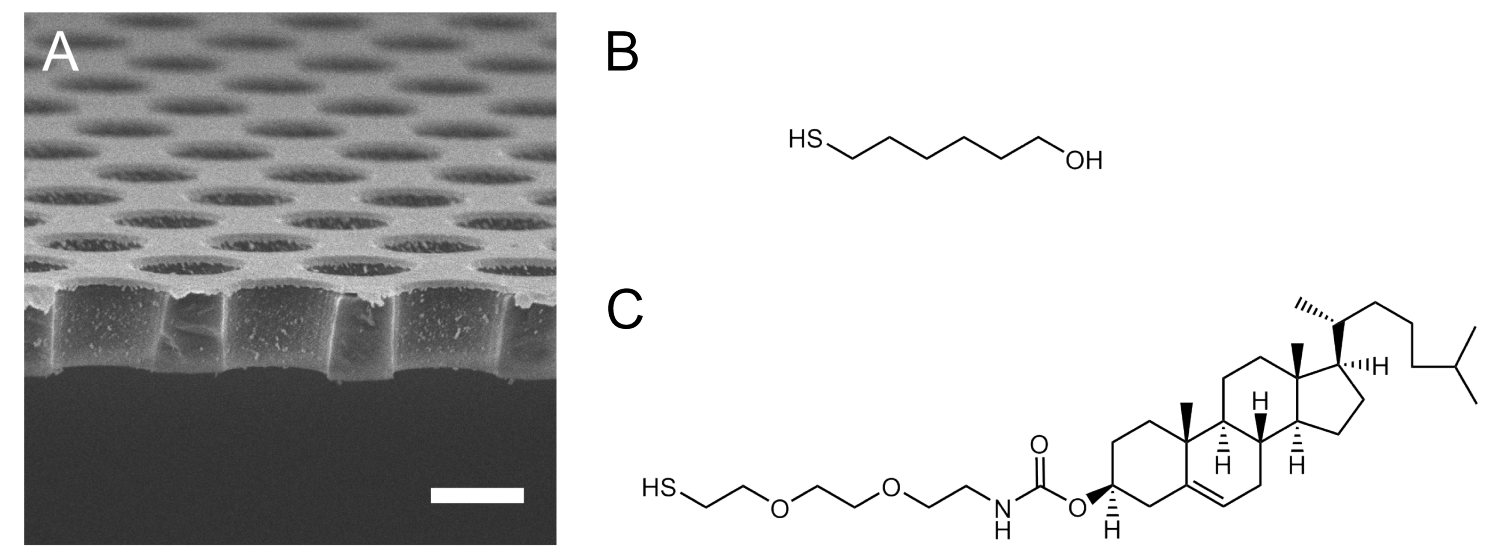

Figure 3.16. (A) Scanning electron micrograph of a gold coated porous substrate. Scale bar: $1 \mu \mathrm{m}$. Structure of the thiols 6-MH (B) and CPEO3 (C) used to prepare densely packed self-assembled monolayers on top of the gold surface.

The functionalized substrates were rinsed with ethanol and MilliQ and subsequently placed in a measuring chamber filled with buffer solution (10 mM HEPES, $100 \mathrm{~mm}$ $\mathrm{KCl}, 1 \mathrm{~mm}$ EDTA, pH 7.4). $10-20 \mu \mathrm{l}$ GUV suspension was then added on top of the porous substrate and incubated for 20-30 min. The GUVs spontaneously rupture and form PSM patches. Residual GUVs and lipid material was washed from the surface by gently rinsing with a pipette. The individual preparation steps are schematically represented in Figure 3.17 .

\subsubsection{Density gradient centrifugation}

Density gradient centrifugation involves the centrifugation of molecules or cell components through a medium of graded density. The material separates within the medium based on the buoyant density when exposed to a elevated centrifugal force. The components with the highest density will be nearest to the bottom, while those with a lower density are located at the top. Density gradient centrifugation was performed to 


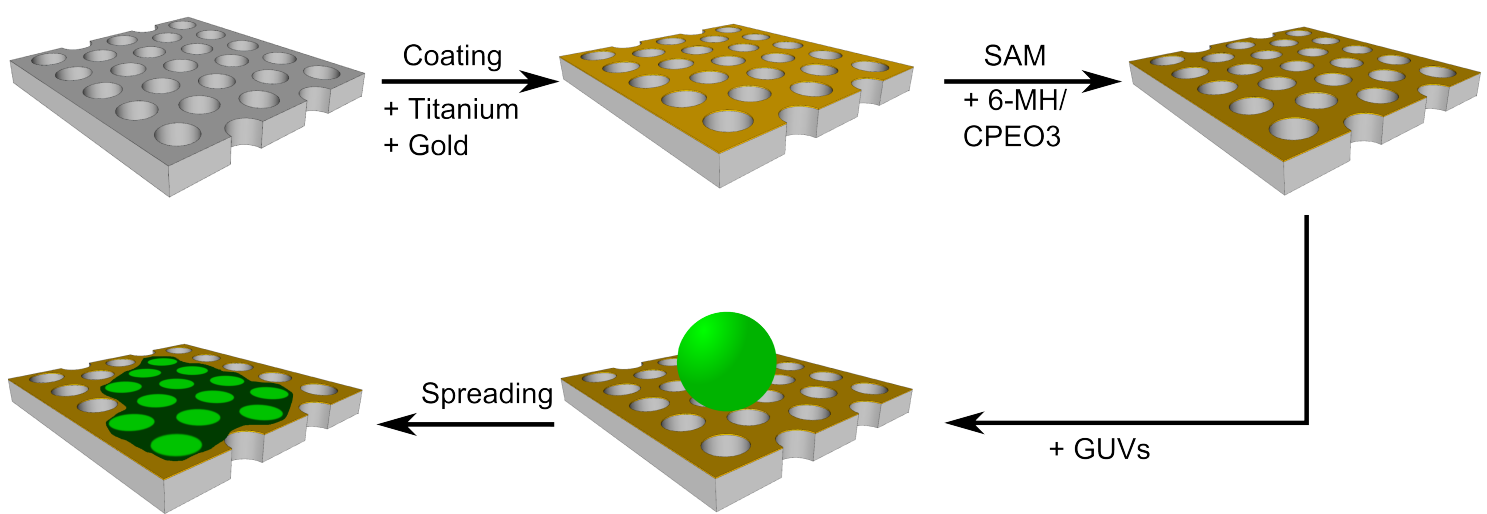

Figure 3.17. Schematic drawing of the individual preparation steps to generate porespanning membranes. Porous substrates are coated with titanium and a thin gold layer and immersed in thiol solutions to assemble a monolayer film. GUVs are spread on top of the monolayer films, which cover part of the porous substrates resembling pore-spanning membranes.

separate proteins and liposomes to analyze the reconstitution efficiency of SNAREs into vesicles. In the gradient, unreconstituted protein will float at the bottom while buffer filled liposomes float to the top.

\section{Experimental procedure}

A continuous Nycodenz gradient ranging from 0-40\% was used for the analysis. Proteovesicle solution $(40 \mu \mathrm{l})$ was mixed with Nycodenz solution $(80 \%, \mathrm{w} / \mathrm{v})(40 \mu \mathrm{l})$ and pipetted into an ultracentrifuge tube. The mixture was carefully overlayed with $(40 \mu \mathrm{l})$ $30 \%$ Nycodenz solution and $(20 \mu \mathrm{l})$ buffer $(10 \mathrm{~mm}$ HEPES, $100 \mathrm{~mm} \mathrm{KCl,} 1 \mathrm{~mm}$ EDTA, $\mathrm{pH}$ 7.4). The sample was then centrifuged for 2.5 hours at $45000 \mathrm{rpm}$ to establish a continuous Nycodenz density gradient. After centrifugation, 6 fractions of $20 \mu$ were collected and further analyzed by SDS-PAGE.

\subsubsection{Ion exchange chromatography}

Ion-exchange chromatography (IEC) was used to purify and concentrate SNARE proteins. IEC separates polar molecules based on their affinity to a stationary ion exchange matrix. Negatively charged molecules are separated on positively charged columns and vice versa. The molecules that travel through the exchange column can reversible bind to small moieties via ionic bonds. By changing the buffer composition, i.e. increasing the ionic strength, the molecules can be displaced from the matrix. This 
allows to separate and concentrate proteins by applying a buffer gradient, starting with low ionic concentrations towards higher ones. In proteins the net charge is caused by acidic and basic amino acids that are protonated or deprotonated at different $\mathrm{pH}$-values. The isoelectric point (IP) is the $\mathrm{pH}$-value at which the protein has no net charge. Above the IP the protein has a negative net charge, while below the PI the protein has a positive net charge. This allows to separate different proteins at a constant $\mathrm{pH}$ in dependence of the ionic strength of the buffer. Proteins with a negative charge (SNAP 25a, syx $1 \mathrm{~A}$ and $\Delta \mathrm{N}$-complex) were purified on MonoQ anion exchange columns, while proteins with a positive net charge like syb 2 and syb 2C79 (residues 49-96) were purified on a MonoS cation exchange column.

\section{Experimental procedure}

All protein samples were diluted with low salt Äkta buffer A below their elution conductivity and sterile filtered prior to the purification on a Äkta protein purification system (GE Healthcare, Little Chalfont, UK). The ion exchange column was equilibrated in Äkta buffer A and the protein sample was loaded onto the column. A buffer gradient composed of Äkta buffer A and B was pumped over the column to elute the proteins. The buffer gradient was adapted for each protein individually as shown in the chromatograms. The Äkta elutions were analyzed in real time by an UV-vis detector $(\lambda=280 \mathrm{~nm})$ and fractionated with an auto sampler (Frac-920, GE Healthcare, Little Chalfont, UK). The fractions were subsequently analyzed by SDS-PAGE to identify the protein.

\subsubsection{SDS-polyacrylamide gel electrophoresis}

Sodium dodecyl sulfat polyacrylamide gel electrophoresis (SDS-PAGE) was used to analyze the molecular weight of proteins. SDS-PAGE is a common technique used to separate proteins and peptides in dependence of their molecular weight in an electric field. The proteins are mixed with the detergent SDS to denature them before they are added onto a polyacrylamide gel. A voltage is applied across the gel causing the proteins to move to the anode. Due to the large excess of negatively charged SDS, the net charge of each protein is masked and the migration speed in an electric field is only a function of the molecular weight of the proteins. 


\section{Experimental procedure}

Polyacrylamide gels $(8.3 \times 7.3 \mathrm{~cm})$ were prepared following a technique of Laemmli using a discontinuous gel that consists of a stacking and a resolving gel [66. The compositions of the buffers and the acrylamdide gels are shown in table 9.13 and 9.14. The $10 \%$ acrylamide gels was prepared by pouring $3.4 \mathrm{ml}$ of resolving gel in a casting chamber (Mini-PROTEAN Multi-Casting Chamber, Bio Rad, CA, USA) and cautiously overlaying it with $1 \mathrm{ml}$ stacking gel. The gels were allowed to polymerize for at least $1 \mathrm{~h}$ before use. The protein samples $(1-3 \mu \mathrm{l})$ were mixed with an equivalent volume $2 \mathrm{x}$ probe buffer and applied on the acrylamide gel, which was mounted in an electrophoresis chamber (Mini-PROTEAN Tetra Vertical Electrophoresis Cell, BioRad, CA, USA). Sufficient amounts of cathode and anode buffer were added to the inner and outer chamber and a voltage of $80 \mathrm{~V}$ was applied across the gel for $20 \mathrm{~min}$. The voltage was subsequently increased to $180 \mathrm{~V}$ for $1 \mathrm{~h}$. The gel was then washed by adding $100 \mathrm{ml}$ distilled water and heating for $30 \mathrm{~s}$ in the microwave. The gel was placed on a shaker for 2 minutes and the washing step was repeated twice with fresh water. Coomassie G-250 staining solution ( $80 \mathrm{mg} / \mathrm{ml}$ Coomassie G-250, $3 \mathrm{ml} \mathrm{HCl}$ ) was then added $(100 \mathrm{ml})$, heated for $30 \mathrm{~s}$ in the microwave and incubated for 5 minutes. The staining solution was poured of and $100 \mathrm{ml}$ distilled water were added, heated in the microwave for $30 \mathrm{~s}$, and incubated for $5 \mathrm{~min}$ to destain the background of the gel.

\subsection{Biophysical methods}

\subsubsection{Spinning disc confocal microscopy (SDCM)}

Spinning disc confocal microscopy (SDCM) is a fluorescence based microscopy technique that allows to image biological samples with a confocal detection and very high recording speeds. Like other fluorescence microscopy techniques, SDCM relies on fluorophores, which are excited by a laser. The fluorophores emit light of a longer wavelength, which is separated from the illumination light by a dichroic mirror and a filter. This allows to visualize fluorescently labeled structures and to measure fluorescence intensities quantitatively. Confocal microscopy can be used to image a specimen three dimensionally by collecting the emitted light from different planes of the sample and rejecting out of plane light by a pinhole. While conventional confocal laser scanning microscopes (CLSMs) use a single laser beam that is scanned over the surface, and a single pinhole to reject the out of plane light, a spinning disc confocal microscope 
is equipped with a rotating disc (Nipkow disc) that harbors several thousand pinholes that are spirally arranged (Fig. 3.18). A second disc with a micro lens array is mounted above the Nipkow disc, which spins synchronously. Laser light is focused by the micro lens array through the pinholes of the Nipkow disc onto the sample. As the disc spins, the sample surface is continuously scanned by the spirally moving light spots. The emitted fluorescence light of the focal plane of the specimen than passes through the pinholes of the Nipkow disc again and is separated by a dichroic beamsplitter from the excitation light and directed to the detector through a filter. The great advantage of SDCM over CLSM is that multiple beams simultaneously illuminate parts of the sample and thus allow to image much faster than with a single laser beam. SDCM was used in this work to image SNARE-mediated fusion on a single vesicle level. The confocal setup was necessary to block out of focus light, which originates from fluorescently labeled vesicles injected over each PSM resulting in a high background signal.

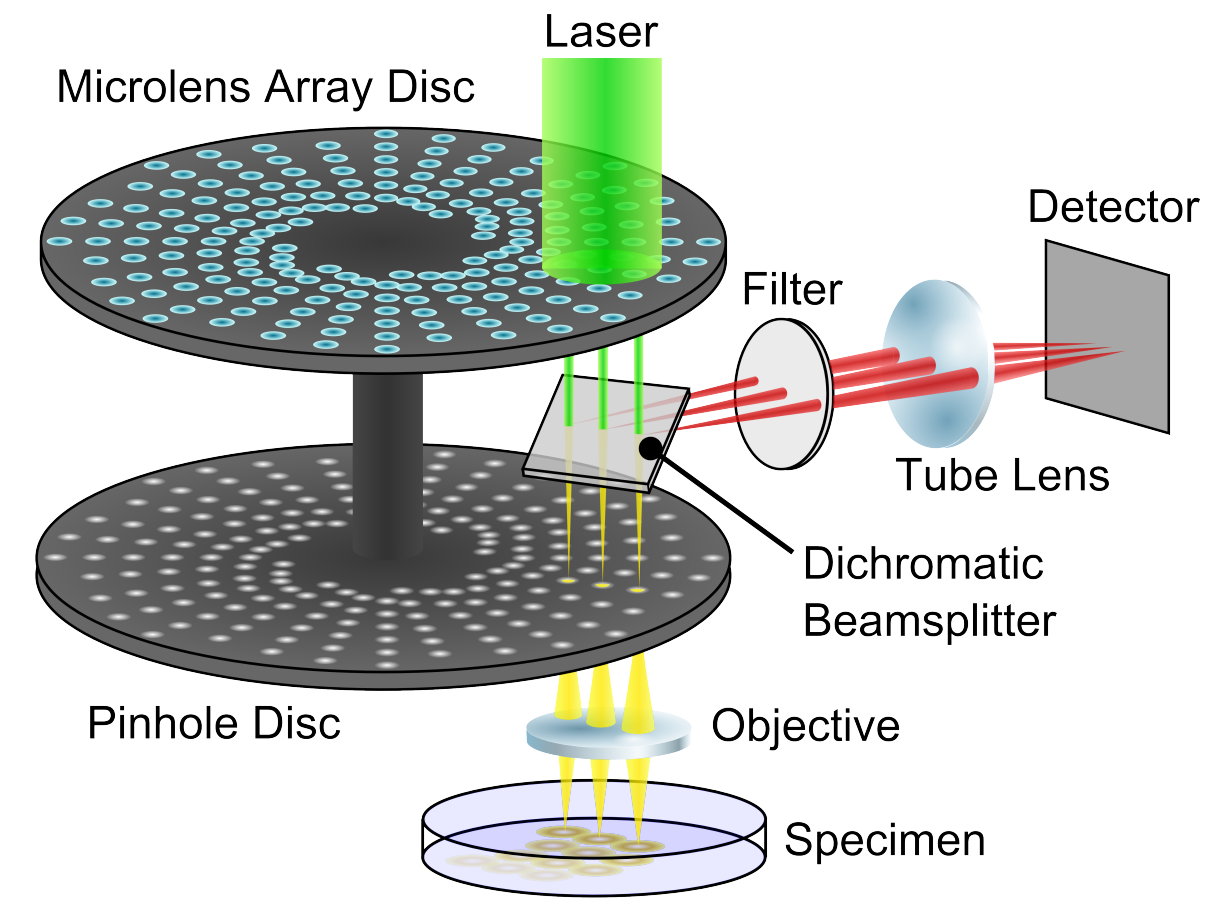

Figure 3.18. Schematic illustration of a spinning disc setup modified according to Gleisner [67]. Laser light is focused by a microlens array through the pinholes of a disc onto the specimen. Excitation and emission light from the fluorescent sample are collected by the objective and focused through the pinhole disc on a dichroic beamsplitter. The beamsplitter separates the emission light from the excitation light and directs it to the camera sensor. 


\section{Experimental procedure}

Single vesicle fusion experiments were performed with an upright spinning disc confocal microscope (spinning disc: Yokogawa CSU-X, Rota Yokogawa GmbH \& Co. KG, Wehr, Germany; stand: Olympus custom-made, Olympus Deutschland GmbH, Hamburg, Germany; camera: iXON 897Ultra, Andor Technology Ltd., Belfast, UK) equipped with a water immersion objective (LUMFLN 60XW 60x NA 1.1, Olympus, Hamburg, Germany). In one-channel mode TexasRed-DPPE was excited at $\lambda=561 \mathrm{~nm}$ and selected with a $590 \mathrm{~nm}$ long pass emission filter (AHF Analysentechnik AG, Tübingen, Germany). In two-channel mode, TexasRed-DPPE was excited at $\lambda=590 \mathrm{~nm}$ and selected with a 650/60 bandpass emission filter (AHF Analysentechnik AG, Tübingen, Germany), while Atto488-DPPE was simultaneously excited at $\lambda=488 \mathrm{~nm}$ and selected with a 525/50 bandpass emission filter (AHF Analysentechnik AG, Tübingen, Germany). Each channel was aligned onto one half of the $512 \times 512$ pixel $^{2}$ detector of the EMCCD camera using an image splitter (Acal BFi Germany GmbH, Dietzenbach, Germany). PSMs were prepared as described in Chapter 3.2.5 and mounted under the microscope. Image acquisition was then started and $0.5-1 \mu \mathrm{l}$ syb 2 vesicle suspension (Chapter 3.2.3 were carefully injected over the focused PSM patch. In one-channel mode, the data was spooled with a recording rate of 95 frames/s, while in twochannel mode the data was spooled with 48 frames/s. Typical recording times for each time series were $155 \mathrm{~s}$. The manual addition of vesicles to each individual PSM patch allowed to record several PSMs per substrate. Time resolved fluorescence intensity traces of fusing vesicles were analyzed manually by placing a region of interest (ROI) of $3 \times 3$ to $4 \times 4$ pixel $^{2}$ on the center of each vesicle. Vesicles fusing with the free standing PSM (f-PSM) were individually tracked with the software TrackNTrace [68].

\subsubsection{Atomic force microscopy (AFM)}

Atomic force microscopy (AFM), invented 1986 by Binning et al., is a scanning probe microscopy technique, providing high resolution imaging and force spectroscopy [69]. AFM can be performed in air and in water, which makes this technique suitable for the analysis of materials and biological specimen. An AFM utilizes a sharp tip attached to the end of a microfabricated cantilever, which is raster-scanned over a surface by moving the sample or the cantilever with piezoactuator (Fig. 3.19). The interation of the cantilever with the surface leads to a deflection of the cantilever. This deflection 
is measured by a light pointer system alligned to the backside of the cantilever and reflected to a four quadrant photodiode.

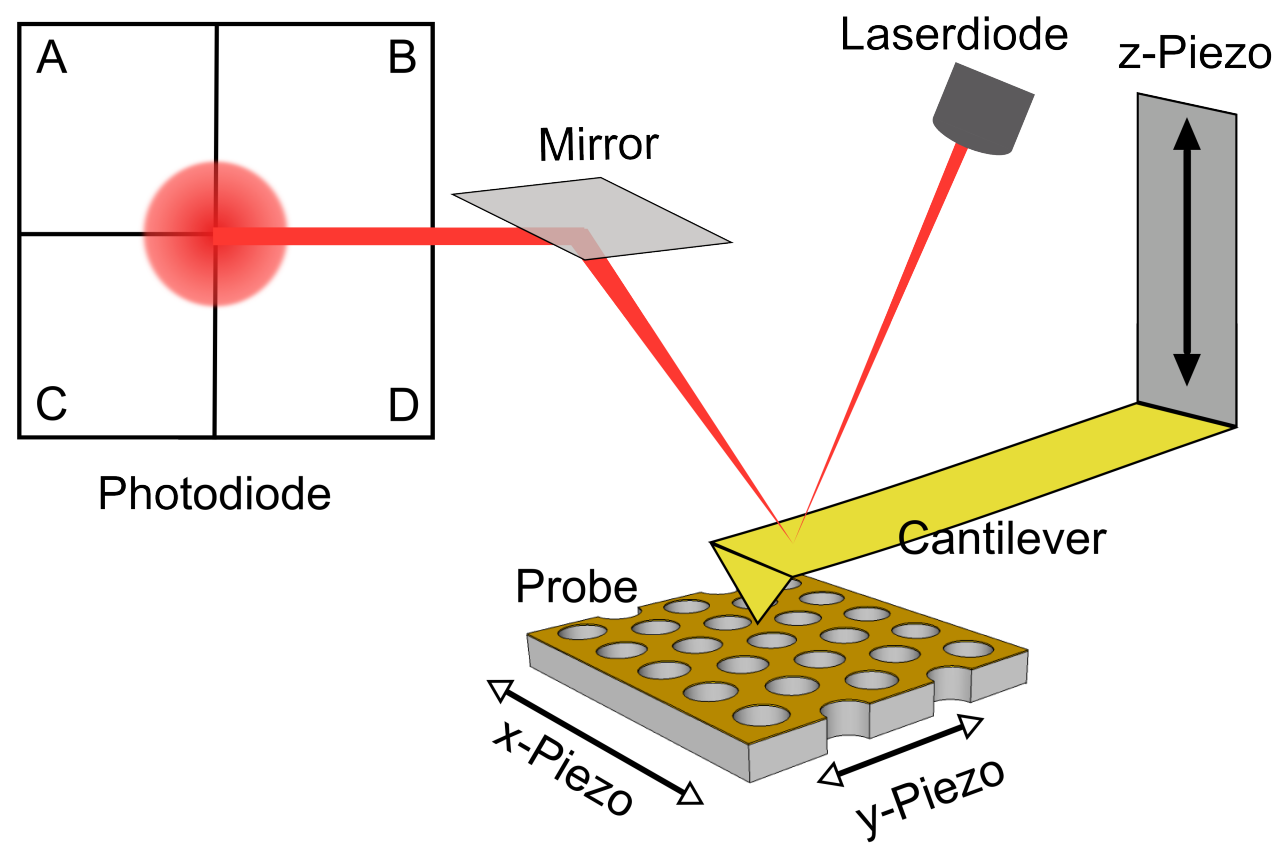

Figure 3.19. Schematic illustration of an atomic force microscope modified according to Mey [70]. A cantilever is raster scanned over the specimen via piezo electric transducer in $\mathrm{x}, \mathrm{y}$ and $\mathrm{z}$ direction. A light spot is focused on the back of the cantilever and the reflected beam is directed onto a four quadrant photodiode, which detects the deflection of the cantilever.

Atomic force microscopy is able to apply and measure very small forces locally, as they occur upon protein unfolding or membrane deformations [71, 72]. In a force measurement, the vertical deflection of the cantilever $Z_{c}$ is measured as a function of the piezoactuator position $Z_{p}$ moving the cantilever with constant velocity up and down (Figure $3.20 \mathrm{~A}$ ). The force acting on the cantilever is proportional to the deflection according to Hooke's law $F=k_{\mathrm{c}} \cdot Z_{c}$ with $k_{\mathrm{c}}$ the spring constant of the cantilever. To obtain force versus distance curves, $Z_{\mathrm{c}}$ and $Z_{\mathrm{p}}$ have to be converted into force and distance (Figure $3.20 \mathrm{~B}$ ). This is done by applying a fit to the constant compliance region to obtain the slop that is equal to the conversion factor of the detector signal (volts) and the deflection of the cantilever (nanometer). The tip-sample distance $D$ can than be calculated by adding up the deflection to the piezo position $D=Z_{\mathrm{p}}+Z_{\mathrm{c}}$. The force $F$ is derived by multiplying the deflection $Z_{\mathrm{c}}$ with the spring constant $k_{\mathrm{c}}$ according to Hooke's law. Although the spring constant can be principally calculated from the material properties and the dimensions of the cantilever, proper thickness 
determination is difficult and small changes in the fabrication process often lead to the fact that calculated spring constant significantly differ from measured ones [73]. This is why spring constants are calibrated prior to each experiment by dynamic methods like the thermal noise method introduced by Hutter and Bechhoefer [74]. The thermal noise method makes use of the fact that the mean thermal energy of a harmonic system at temperature $T$ is equal to $k_{\mathrm{B}} / 2$ per degrees of freedom. A cantilever can be seen as a harmonic oscillator for very small amplitudes $(0.1 \mathrm{~nm})$ with two degree of freedom (deflection and rotation), therefore the mean square deflection $\left\langle x^{2}\right\rangle$ of the cantilever generated by thermal fluctuation fulfills:

$$
\left\langle x^{2}\right\rangle=\frac{k_{\mathrm{B}} T}{k_{\mathrm{c}}},
$$

with $T$ the temperature and $k_{\mathrm{B}}$ the Boltzmann constant. The thermal fluctuations can be determined by measuring the deflection of the cantilever far away from a surface for a couple of seconds. Fast Fourier transformation calculates the power spectrum $P(\nu)\left(\left\langle x^{2}\right\rangle\right.$ vs. frequency) in which the first resonance peak can be fitted to obtain the total value of $\left\langle x^{2}\right\rangle$. It must be considered that the thermal fluctuations obtained by this method $\left(\left\langle x^{* 2}\right\rangle\right)$ are systematically underestimated as only the first resonance peak of the power spectrum is evaluated and higher oscillation modes are neglected [75]. To correct for this issue, a proportionality factor $\alpha$ is introduced to calculate the spring constant according to:

$$
k_{\mathrm{c}}=\frac{\alpha k_{\mathrm{B}} T}{\left\langle x^{* 2}\right\rangle} .
$$

For v-shaped cantilevers, a proportionality factor of $\alpha=0.78$ is used. This factor also compensates for the fact that the optical lever system detects the inclination of the cantilever upon measuring a thermal spectrum rather than its deflection, which occurs with a vertical load on the tip [76].

\section{Experimental procedure}

Pore-spanning membranes composed of DPhPC and 1 mol\% TR-DPPE prepared on porous substrates with pore diameters of $1.2 \mu \mathrm{m}$ were prepared as described in Chapter 3.2.5 and investigated by a MFP-3D AFM (Asylum Research, Santa Barbara, CA). The AFM was equipped with a top view camera to localize the position of individual PSMs. Force volume images with a size of $6 \mathrm{x} 6 \mu \mathrm{m}^{2}$ and a resolution of 32x32 $\mathrm{pixel}^{2}$ were recored with a load force of $400 \mathrm{pN}$ and a velocity of $3 \mu \mathrm{m} \mathrm{s}^{-1}$ using MLCT-Au 

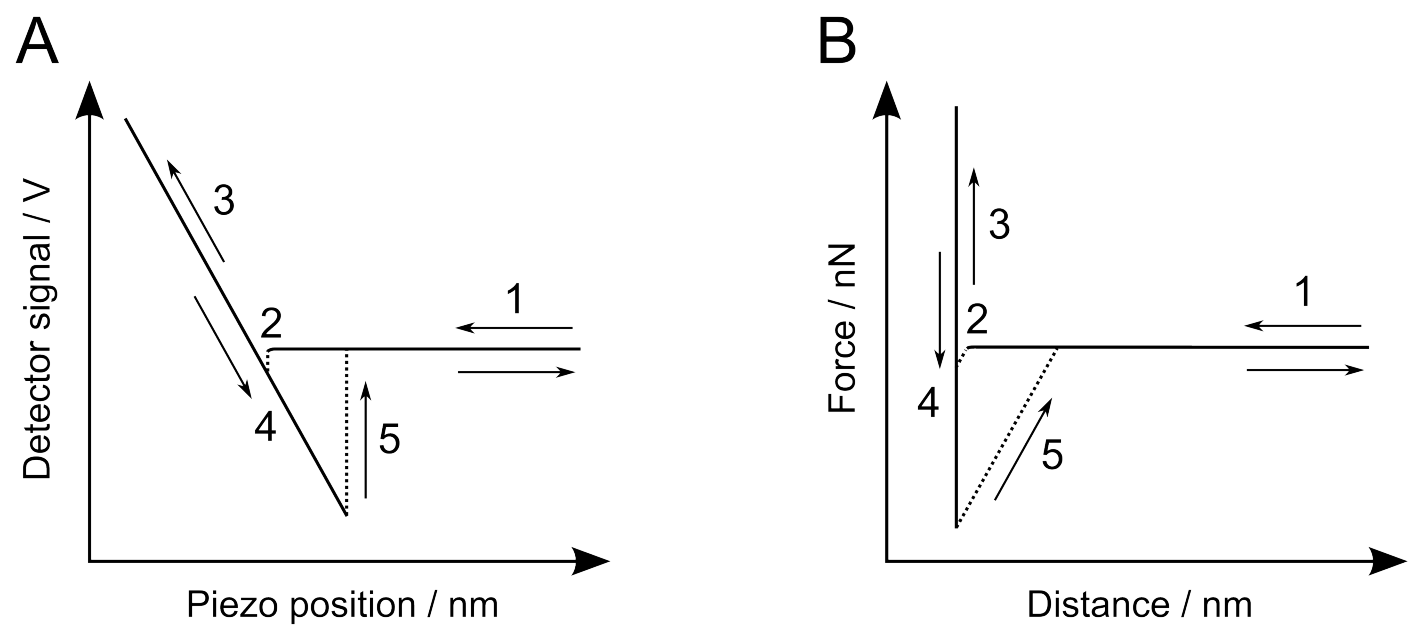

Figure 3.20. Detector signal versus piezoactuator position (A) and force versus distance curve (B) derived from (A). The different regions are numbered within the images. The regime where the cantilever is not in contact with the surface is called zero force region (1). When the cantilever approaches the surface, attractive forces (van der Waals) pull down the cantilever (2). At the approach part of the constant compliance region, probe and surface move in parallel (3). At the end of the retract part of the constant compliance region, adhesion leads to negative bending (4). When the restoring forces exceed the adhesion forces the cantilever jumps away from the surface (5).

cantilever (Bruker AFM Probes, Calle Tecate, USA) with a nominal spring constant of $10 \mathrm{mN} \mathrm{m}^{-1}$ (Fig. 3.21). The exact spring constant of each cantilever was determined prior to each experiment with the thermal noise method integrated in the Asylum Research software based on IGOR Pro (Wavemetrics, Oregon, USA). For the statistical analysis of the apparent spring constants $k_{\text {app }}$, from which the lateral membrane tension can be extracted, force-indentation curves were evaluated by picking four curves from the center of each pore-spanning membrane. 

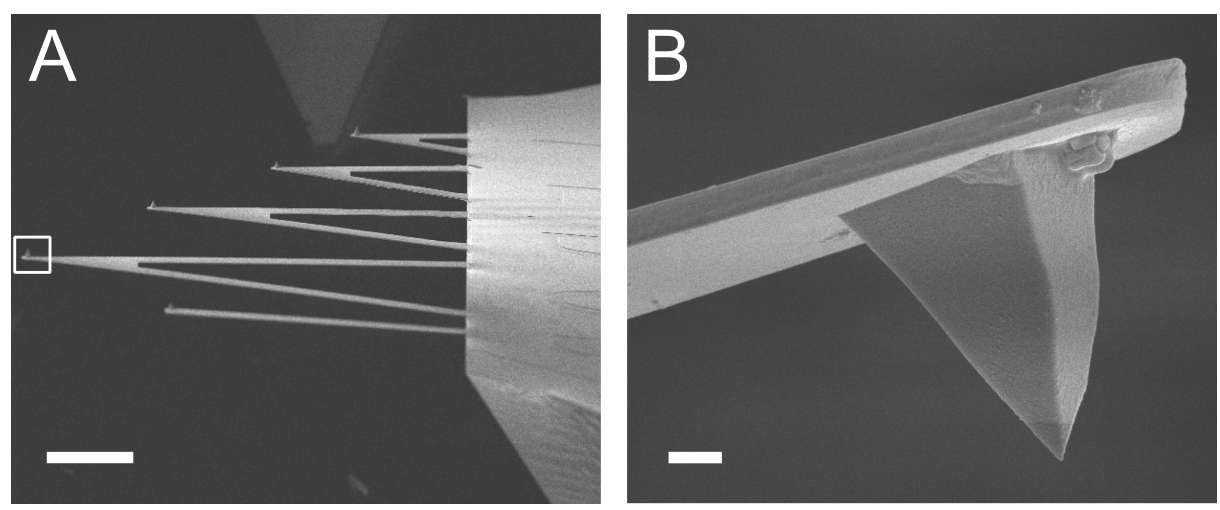

Figure 3.21. Scanning electron micrographs of a MLCT-Au cantilever chip. (A) The chip harbors several cantilevers at its end with different spring constants. For the force indentation experiments the largest v-shaped cantilever (marked by white box) was used. Scale bar: $50 \mu \mathrm{m}$. (B) Magnification of the tip of the large cantilever (white box) used for the force indentation experiments. Scale bar: $1 \mu \mathrm{m}$

\subsubsection{Polarization modulation infrared reflection-absorption spectroscopy (PM-IRRAS)}

Polarization modulation infrared reflection-absorption spectroscopy (PM-IRRAS) is a surface sensitive spectroscopy technique suitable to probe thin polymer films or selfassembled monolayers on metallic surfaces. The spectra in PM-IRRAS are comparable to standard transmission IR spectra, allowing to compare the obtained data with databases and assign specific absorption bands. At grazing angles of incidence $\left(>80^{\circ}\right)$, the intensity of a reflected p-polarized infrared light beam is enhanced at a metal surface, allowing to excite the thin adsorbed molecular film (Fig. 3.22). Under the same conditions, a reflected s-polarized infrared beam has virtually no intensity at the metal surface. The predominance of p-polarized light over s-polarized light at the metal surface can be used to obtain background corrected spectra in real time by polarization modulation. The background in PM-IRRAS is mainly dominated by $\mathrm{CO}_{2}$ and $\mathrm{H}_{2} \mathrm{O}$ molecules in the measuring chamber of the instrument. These molecules are isotropically orientated in the gas and absorb both s- and p- polarized infrared light. By changing the polarization of the infrared light between s- and p-polarization with a photoelastic modulator, an absorption spectrum of the $\mathrm{CO}_{2}$ and $\mathrm{H}_{2} \mathrm{O}$ molecules without the adsorbed molecules and one with both, the adsorbate and the $\mathrm{CO}_{2}$ and $\mathrm{H}_{2} \mathrm{O}$ molecules can be recorded within a very narrow time frame. From the two spectra, a differential spectrum can be derived, which only contains the signals from the adsorbate. The polarization modulation technique drastically increases the 
sensitivity of the IRRAS measurement and allows to obtain infrared spectra with high quality.

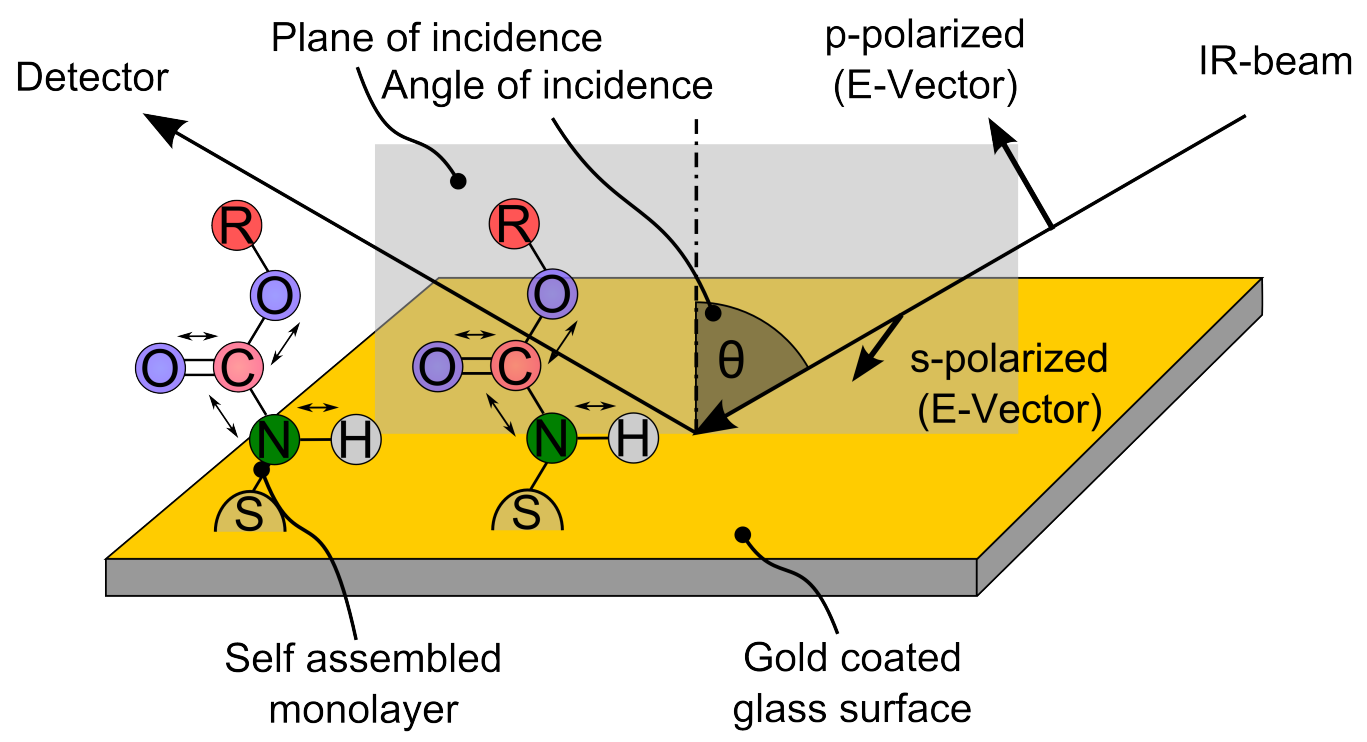

Figure 3.22. Operating principle of PM-IRRAS. An IR-beam is modulated between s- and p-polarization and reflected from the metal surface under grazing angle to the detector. Only the p-polarized light can be absorbed from the self-assembled monolayer on the surface. The isotropic orientated $\mathrm{CO}_{2}$ and $\mathrm{H}_{2} \mathrm{O}$ molecules absorb both s- and p- polarized light, which can be utilized to cancel out the interfering effects of these molecules by generating a differential spectra.

\section{Experimental procedure}

PM-IRRAS samples were prepared by cleaning glass slides $(1 \mathrm{x} 2 \mathrm{~cm})$ with ethanol and drying them under a stream of nitrogen. The cleaned substrates were sputter coated with a thin titanium, then subsequently coated with a 300 nanometer thick gold layer by thermal evaporation and immediately incubated in $1 \mathrm{mM} n$-propanolic solutions of 6-MH and CPEO3 over night. The samples were rinsed with ethanol and dried under a stream of nitrogen prior to each measurement and subsequently mounted in the external beam of a Fourier-transform infrared (FT-IR) spectrometer (Vertex 70, Bruker, Calle Tecate, USA) equipped with a grid polarizer and a ZnSe photoelastic modulator (PM90, HINDS Instruments, Oegon, USA). All measurements were performed at an angle of incidence of $83^{\circ}$. The reflected IR beam was focused on a liquid nitrogen cooled mercury cadmium telluride (MCT) detector. For each 
measurements, 367 spectra were collected with a resolution of $4 \mathrm{~cm}^{-1}$. The differential spectra were processed and transferred into absorption spectra with the software OPUS 6.5 and a PM-IRRAS script (Bruker, Calle Tecate, USA).

\subsubsection{Contact angle measurements}

Static contact angle measurements of sessile water droplets were performed to investigate the wettability of binary self-assembled monolayers. The wettability of a surface describes the tendency of a drop to spread over the surface. If the drop easily spreads on the surface, the angle at which the solid-liquid interface meets the liquid-vapor interface, called contact angle, is small (Fig. 3.23). In case of a water droplet, high wetting (low contact angle) is caused by hydrophilic surfaces, while low wetting (high contact angle) is caused by hydrophobic surfaces. The contact angle of a water droplet is therefore a measure for the hydrophilicity of a surface.

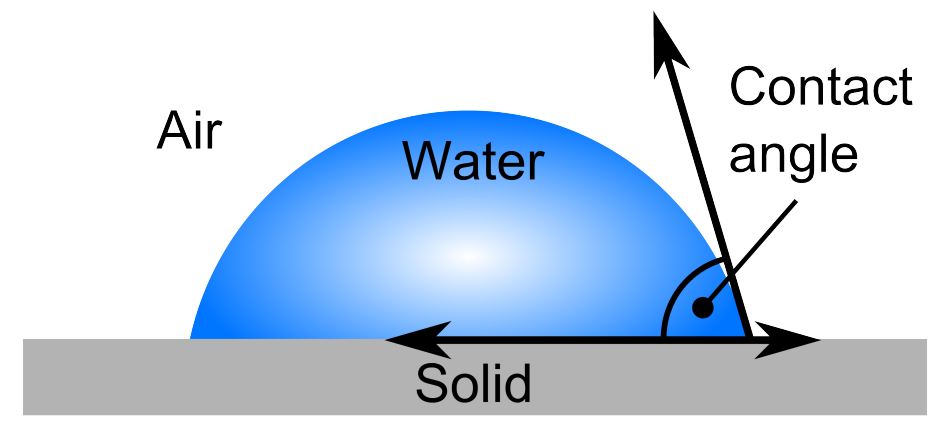

Figure 3.23. Schematic drawing of a sessile water droplet on a solid surface. The angle that connects the solid-gas interface and the solid-liquid interface is the contact angle.

\section{Experimental procedure}

A custom-build contact angle goniometer setup was used to measure the contact angle of a sessile water droplet on top of different gold coated glass slides functionalized with SAMs. The substrates were prepared as those used for the PM-IRRAS measurements (Chapter 3.3.3). The contact angle measurements were performed directly after rinsing each substrate with ethanol and drying it under a stream of nitrogen to exclude surface contamination of the SAMs. $5 \mu$ of ultrapure water were deposited on top of the samples and imaged with a camera (Nikon D600, Nikon) equipped with a AF-S Nikkor 18/300 mm objective (Nikon) fronted by a custom-made lens (Scientific Precision 
Instruments GmbH, Oppenheim, Germany). The contact angle was determined by fitting the Young-Laplace equation to the contour of each water droplet with the ImageJ plugin Drop Shape Analysis [77]. 



\section{Results: Lateral membrane tension of pore-spanning membranes}

Pore-spanning membranes (PSMs) resemble an in vitro model membrane systems with unique mechanical properties, which can be investigated on a local scale with force spectroscopy [63, 78]. PSMs are composed of free-standing membranes, called f-PSM that are spanned over a highly ordered porous micro array and supported membranes, called s-PSMs, on the rim. The porous surface is chemically functionalized with a self-assembled monolayer (SAM) to ensure proper PSM formation and stability. The free energy difference between the s-PSM adhering to the SAM-covered surface and the f-PSM freely spanning the pores, leads to a significant lateral membrane tension in the f-PSM [62, 63]. This makes PSMs very interesting to investigate tension dependent processes like fusion and fission. While PSMs prepared on hydrophobic SAMs, e.g. octadecanethiol (OT), exhibit very high lateral membrane tensions $\left(20-30 \mathrm{mN} \mathrm{m}^{-1}\right)$, those prepared on hydrophilic SAMs, e.g. 2-mercaptoethanol (2-ME), have significant lower lateral tensions $\left(0.5-3 \mathrm{mN} \mathrm{m}^{-1}\right)$ [5, 62].

The goal of this work was to establish PSMs on binary mixed SAMs containing different amounts of hydrophilic 6-mercaptohexanol (6-MH) and hydrophobic cholesterylpolyethylenoxythiol (CPEO3) to investigate the impact of the surface functionalization on the lateral membrane tension of PSMs. 6-MH and CPEO3 were utilized, as the binary mixture of a SAM assembeld from these thiols allows the hydrophobic cholesterol residue of the CPEO3 to insert into the hydrophobic core of the lower lipid leaflet, while the shorter 6-MH SAMs support the lipid headgroups (Fig. 4.1) [5, 79, 80. The formation of binary 6-MH/CPEO3 SAMs was analyzed in detail first by contact angle and grazing angle infra-red spectroscopy. The stability of the PSMs was then further characterized on different SAMs and the lateral membrane tension systematically 
analyzed by force indentation experiments. Parts of this work are published in the Journal Langmuir 64].

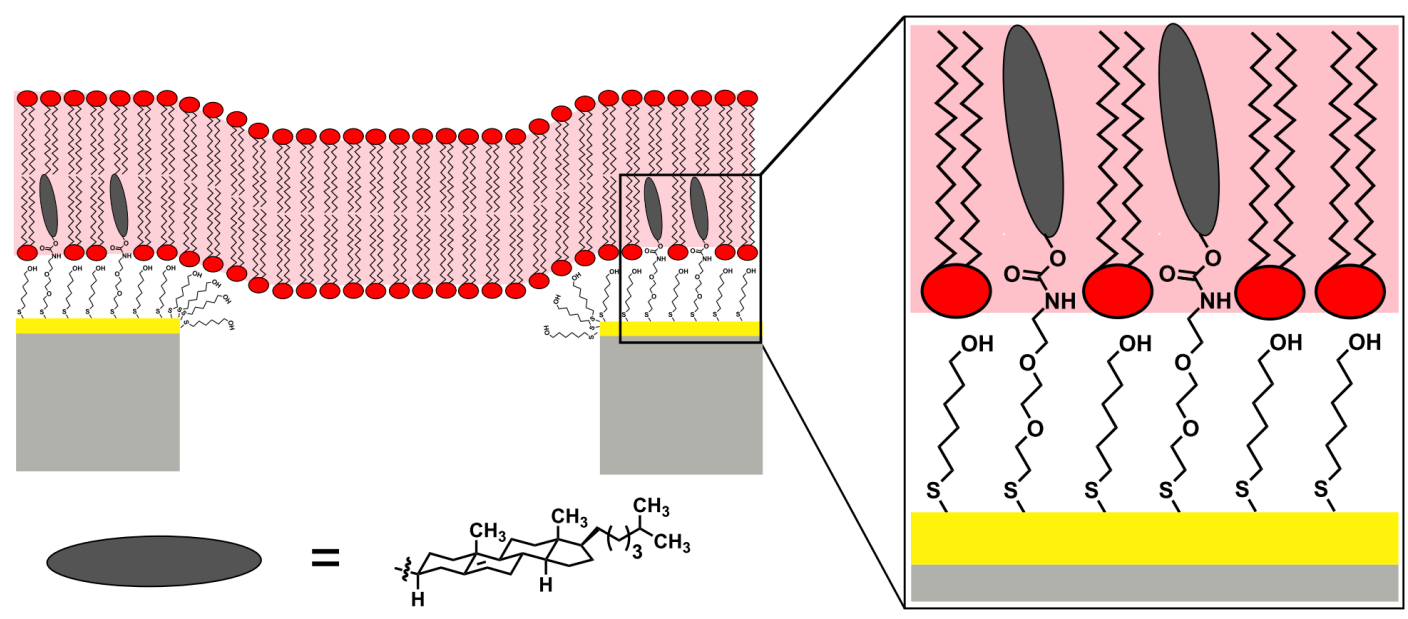

Figure 4.1. Illustration of a membrane spanning a pore on a binary SAM composed of 6-MH and CPEO3. The ethoxy spacer of the CPEO3 is designed to facilitate an insertion of the cholesterol headgroup into the hydrophobic part of the membrane.

\subsection{Wettability of 6-MH/CPEO3 self-assembled monolayers on gold}

Binary SAMs were prepared by exposing gold coated glass substrates to mixtures of 6-MH/CPEO3 dissolved in $n$-propanol (see 3.2.5). Although the ratio of 6-MH/CPEO3 in solution determines the composition of the SAM, it does not lead to the same ratio in the SAM due to complex binding kinetics [81, 82]. In order to analyze the SAM composition in detail and to gather information about the surface properties, different 6-MH/CPEO3 SAMs obtained from different solution mixtures were investigated by static contact angle measurements. Contact angle measurements provide information about the wettability of a surface. While hydrophilic SAMs result in small contact angles $\left(<90^{\circ}\right)$ and therefore in a high wettability, hydrophobic SAMs lead to high contact angles $\left(>90^{\circ}\right)$ of the water droplet. To infer the SAM composition obtained from different solutions, contact angles on 6-MH/CPEO3 SAMs were measured and plotted over the mole fraction of CPEO3 (Fig. 4.2A/B). One finds low contact angles $\left(19^{\circ}\right)$ on SAMs only composed of $6-\mathrm{MH}$, which is indicative of a hydrophilic surface. In contrast, contact angles on SAMs assembled from 6-MH/CPEO3 mixtures are significantly larger, displaying a lower hydrophilicity as a result of the hydrophobic 
cholesterol residues of the CPEO3. The contact angles obtained on the binary SAMs increase monotonically with higher amounts of CPEO3 in the incubation solution with a maximum at $110^{\circ}$ at $15 \mathrm{~mol} \%$ CPEO3. Higher amounts of CPEO3 in the incubation than $15 \mathrm{~mol} \%$ do not result in higher contact angles giving rise to a saturation of the SAM with CPEO3. Even a SAM which only consists of CPEO3 does not show a higher contact angle than a SAM obtained from a mixture of 6-MH/CPEO3 with $15 \mathrm{~mol} \%$ CPEO3. This phenomenon is discussed in detail in Chapter 5.1 .

Contact angle measurements were successfully used to analyze the wettability of binary SAMs composed of 6-MH and CPEO3 to precisely derive their composition. It was ascertained, that the composition of binary 6-MH/CPEO3 SAMs can be specifically altered by the ratio of the thiols in solution. The results also indicated a saturation of the SAM with CPEO3 at 15 mol\% CPEO3 in the binary incubation solution.
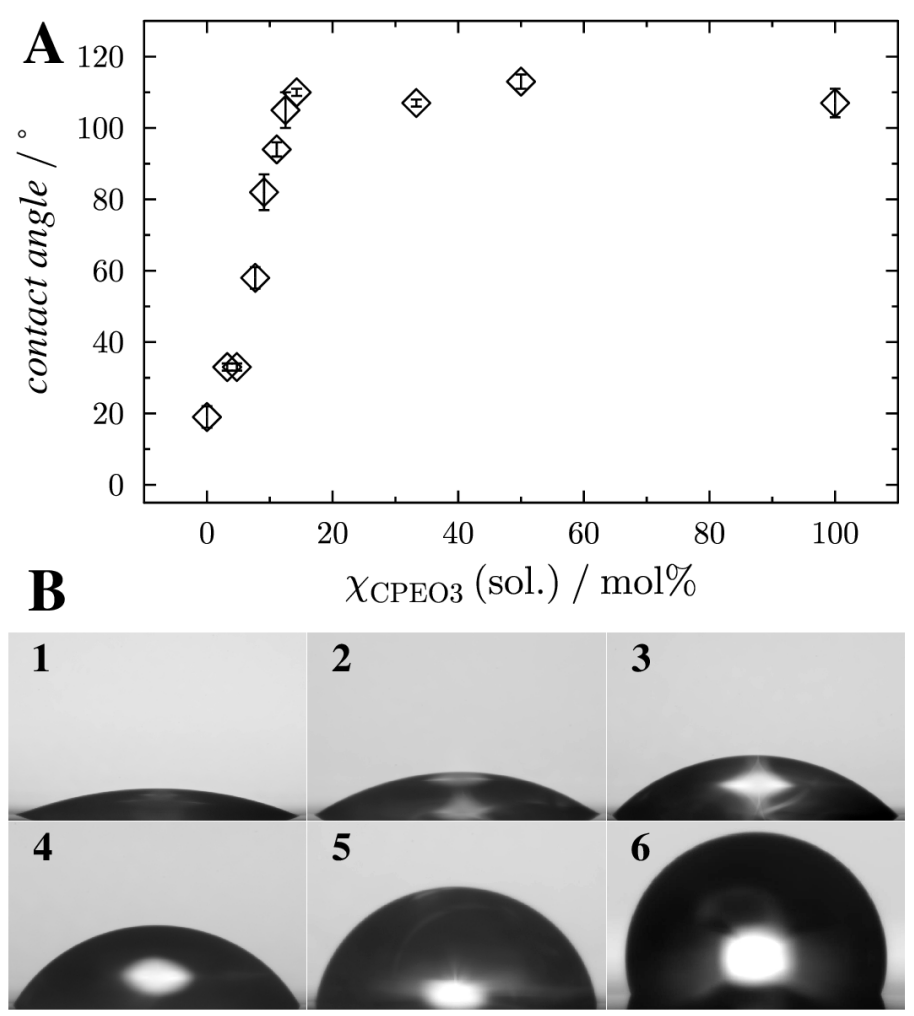

Figure 4.2. (A) Contact angles of sessile water droplets on SAMs composed of different ratios of 6-MH and CPEO3. (B) Contours of water droplets on SAMs of 6-MH and CPEO3 on gold coated glass slides prepared in solutions with mole fractions of CPEO3 of (1) $0 \mathrm{~mol} \%$, (2) $4.8 \mathrm{~mol} \%$, (3) $6.3 \mathrm{~mol} \%$, (4) $7.7 \mathrm{~mol} \%$, (5) $9.1 \mathrm{~mol} \%$, and (6) $100 \mathrm{~mol} \%$ 


\subsection{Chemical analysis of $6-\mathrm{MH} / \mathrm{CPEO}$ self-assembled monolayers on gold}

Binary 6-MH/CPEO3 SAMs were analyzed by polarization modulation infra-red reflection absorption spectroscopy (PM-IRRAS) under grazing angle to gather chemical information of the SAM. PM-IRRAS allows to obtain infra-red absorption spectra from very thin films like SAMs on reflective surfaces. Due to surface selection rules, PM-IRRAS allows to investigate structural changes of the molecules with respect to the surface normal. Gold coated glass slides were incubated in n-propanolic 6-MH/CPEO3 solutions with different mole fractions of CPEO3 and analyzed via PM-IRRAS. Figure 4.3A depicts absorption spectra obtained from 6-MH/CPEO3 SAMs assembled from solutions with different mole fractions of CPEO3. A pure 6-MH SAM (0 mol\% CPEO3)displays characteristic symmetric and asymmetric $\mathrm{CH}_{2}$ stretching vibrations in the high frequency region at $2850 \mathrm{~cm}^{-1}$ and $2919 \mathrm{~cm}^{-1}$, respectively. In case of a binary mixture of $6-\mathrm{MH}$ and CPEO3, the successful incorporation of CPEO3 is indicated by additional absorption bands appearing at $2878 \mathrm{~cm}^{-1}$ and $2961 \mathrm{~cm}^{-1}$, which originate from the symmetric and asymmetric $\mathrm{CH}_{3}$ stretching vibrations. In addition, in the low frequency region, absorption bands from the carbamate group at $1723 \mathrm{~cm}^{-1}$ (C=O stretching), $1532 \mathrm{~cm}^{-1}$ (N-H bending), $1270 \mathrm{~cm}^{-1}$ (C-N stretching) and $1124 \mathrm{~cm}^{-1}$ (C-O stretching) can be observed. The intensities of the $\mathrm{CH}_{2}$ and $\mathrm{CH}_{3}$ stretching bands steadily increase with higher amounts of CPEO3 in the incubation solution, proving that the SAMs contains larger amounts of CPEO3. This observation is in agreement with the results from the contact angle measurements, which also indicated monotonic increase in the wettability due to higher amounts of CPEO3 in the SAM. The absorption bands of the carbamate group increase in a similar fashion. The specific $\mathrm{C}=\mathrm{O}$ stretching vibration at $1723 \mathrm{~cm}^{-1}$ can be used as an indicator for the amount of CPEO3 in the SAM. To visualize changes of the SAM composition, the intensity of the $\mathrm{C}=\mathrm{O}$ absorption band was plotted over the mole\% of CPEO3 in the binary solution (Fig. 4.3B). One finds an increase in the intensity of the absorption band with higher amounts of CPEO3 in solution until a maximum at 20 mol\% CPEO3 is reached displaying a saturation of the SAM with CPEO3.

The assembly of binary 6-MH/CPEO3 SAMs on gold surfaces was successfully analyzed with PM-IRRAS. The absorption spectra verified the proper assembly of 6-MH/CPEO3 SAMs. A saturation of the binary SAM with CPEO3 was observed at 20 mol\% CPEO3 
in solution, which is in good agreement with the saturation point determined by contact angle measurements (15 mol\% CPEO3).
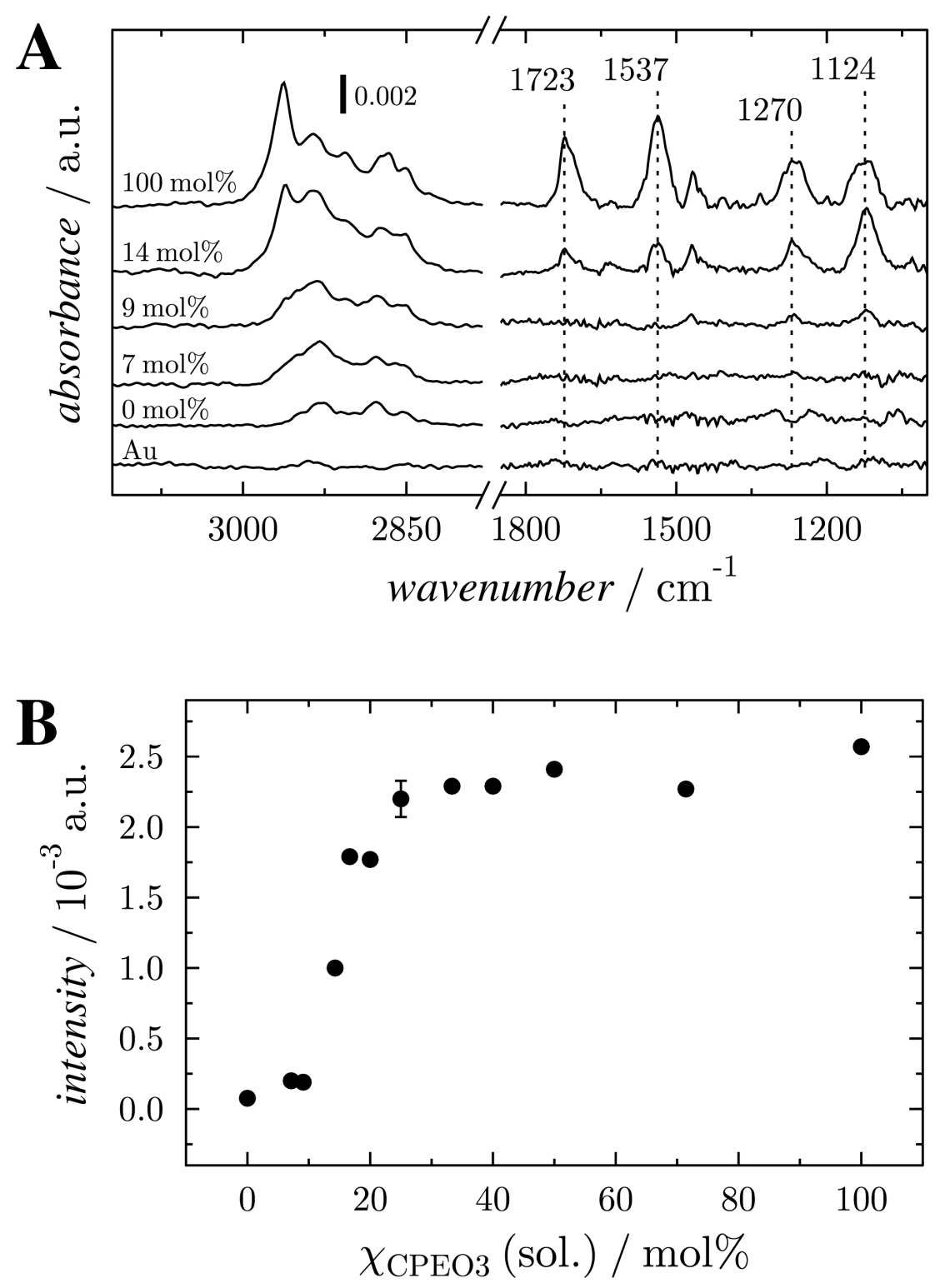

Figure 4.3. (A) PM-IRRAS spectra obtained from binary 6-MH/CPEO3 SAMs prepared from solutions containing different mole fractions of CPEO3. The spectrum of the gold surface serves as a blank measurement. (B) Plot of the intensity of the $\mathrm{C}=\mathrm{O}$ band versus the mole fraction of CPEO3 used in the thiol solution. A typical error bar is shown. 


\subsection{Stability of pore-spanning membranes on binary 6-MH/CPEO3 self-assembled monolayers}

Giant unilamellar vesicles (GUVs) composed of DPhPC were fluorescently labeled with $1 \mathrm{~mol} \%$ TexasRed-DPPE (TR) and spread on porous substrates with a pore diameter of $1.2 \mu \mathrm{m}$ to prepare PSMs. The gold coated porous substrates were foremost incubated in $n$-propanolic solutions of $6-\mathrm{MH} / \mathrm{CPEO} 3$ with $\mathrm{CPEO} 3$ mole fractions ranging of $0-15 \mathrm{~mol} \%$ to investigate the stability of PSMs on the individual SAMs.

Figure 4.4 depicts typical fluorescence images of three PSMs patches recorded 30 minutes after GUV spreading on SAMs containing $4.8 \mathrm{~mol} \%$ (A), $7.1 \mathrm{~mol} \%$ (B) and $9.1 \mathrm{~mol} \%$ CPEO3 (C). Red fluorescence indicates intact bilayers spanning the pores. The fluorescence on the pore rims is not visible as it is quenched by the underlying gold film. In case of PSMs on SAMs assembled from mixtures containing up to $4.8 \mathrm{~mol} \%$ CPEO3, all pores occupied by the patch are spanned by membranes and are stable over several hours. In contrast, PSMs on SAMs assembled from solutions with more than 4.8 mol\% CPEO3 (Figure $4.4 \mathrm{C} / \mathrm{D}$ ) showed many pores which are not spanned by a membrane anymore after 30 minutes indicating spontaneous rupturing of the membranes over time. On SAMs, prepared from solutions with more than $10 \mathrm{~mol} \%$ CPEO3, the membranes ruptured within seconds or even during the spreading process rendering those preparations useless for further investigations. The stability of PSMs composed of physiologically more relevant lipid mixtures (POPC/cholesterol (8:2)) and (DOPC, POPS, POPE, cholesterol (5:1:2:2)) on the binary SAMs was additionally analyzed. It turned out that the stabilities of these PSMs on SAMs containing CPEO3 were even more reduced compared to those composed of pure DPhPC. The reason for this observations are discussed in Chapter 5.2 . 

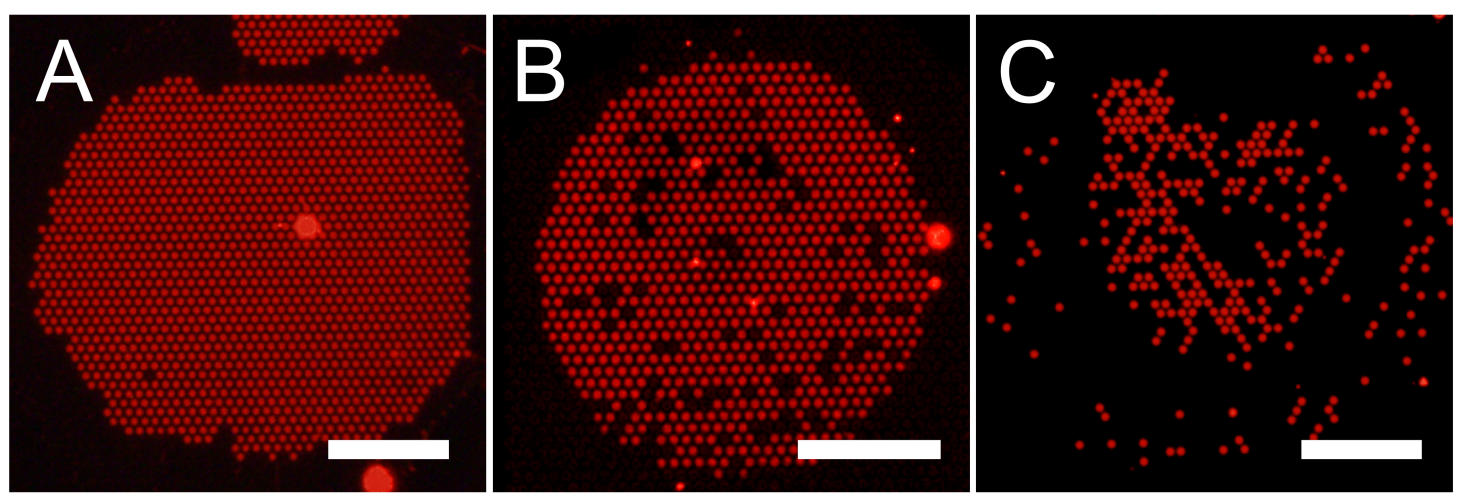

Figure 4.4. Fluorescence images of pore-spanning DPhPC membranes labeled with 1 mol\% TR recorded 30 minutes after GUV spreading on mixed SAMs prepared from $6-\mathrm{MH} / \mathrm{CPEO} 3$ solutions with $\chi_{\mathrm{CPEO} 3(\mathrm{sol})}=4.8 \mathrm{~mol} \%$ (B), $\chi_{\mathrm{CPEO}}(\mathrm{sol})=7.1 \mathrm{~mol} \%(\mathrm{C})$, and $\chi_{\mathrm{CPEO} 3(\mathrm{sol})}=9.1 \mathrm{~mol} \%$ (D). Scale bars: $20 \mu \mathrm{m}$.

\subsection{Lateral membrane tension of pore-spanning membranes on 6-MH/CPEO3 self-assembled monolayers}

The lateral membrane tension of PSMs spanned over pores with a diameter of $1.2 \mu \mathrm{m}$ on binary SAMs composed of different ratios of 6-MH/CPEO3 was analyzed by force indentation experiments. Force indentation experiments were performed by indenting the PSMs and measuring the applied force as a function of the cantilever tip and membrane separation. The obtained force-distance (FD) curves reflect the overall force response of the membrane during indentation and allow to derive the lateral membrane tension [62, 63, 83. To probe large parts of the PSM and assign the individual FD curves, force volume maps of evenly spaced 32x32 FD-curves were recorded on individual PSM patches. A typical force volume map obtained on a PSM on a pure 6-MH SAM is depicted in Figure 4.5A. Each pixel codes the information of a full FD curve including trace and retrace. The gray scale visualizes the relative depth of the trigger point obtained in each curve. The force maps allow to easily distinguish between supported (black dot) and pore-spanning membranes (red dot) as well as uncovered pores (blue dot) by the different trigger point depths. FD curves depicted from such different indentation sites are displayed in Figure 4.5B. The height at which the cantilever gets into contact with the pore rim is arbitrarily set to $0 \mathrm{~nm}$ acting as a reference level.

Supported membranes on the pore rims can not be indented due to the hard $\mathrm{Si}_{3} \mathrm{~N}_{4}$ 

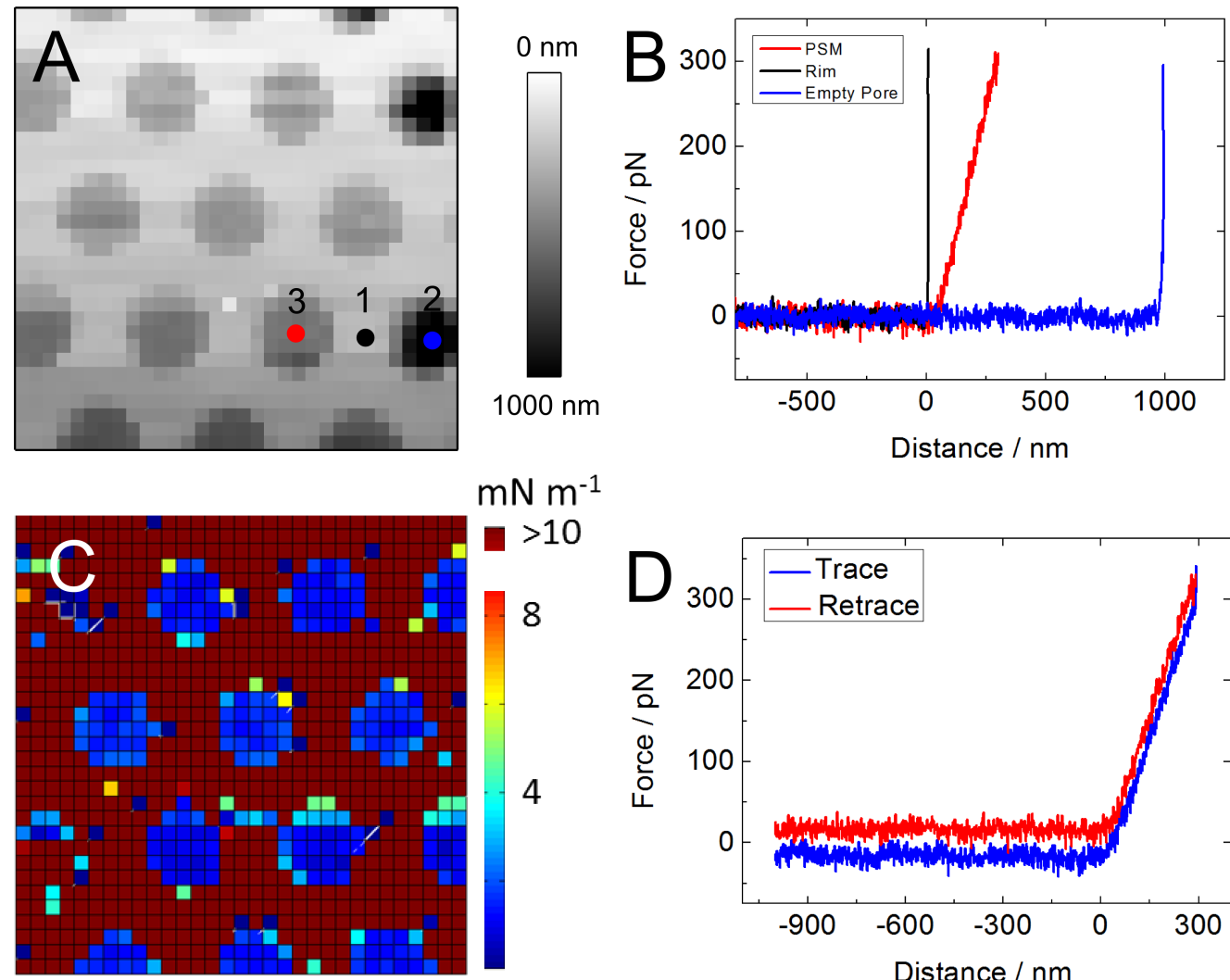

$\mathrm{mN} \mathrm{m}^{-1}$
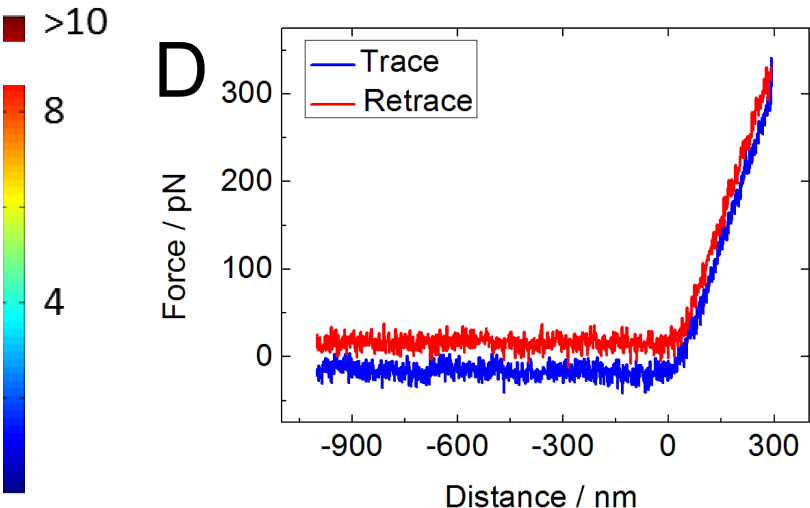

Figure 4.5. Force map measured on a PSM on 6-MH SAM (A). Areas of different trigger point depths are coded by different colors. Indentation sites including the rim (1), an uncovered pore (2) and a PSM (3) are marked by colored dots. Typical FD-curves obtained on the respective indentation sites are shown in (B). A $k_{\text {app-map derived from }}$ the slopes of FD-curves recorded in $(\mathrm{A})$ is shown in $(\mathrm{C})$. Slopes with a greater slope than $10 \mathrm{mN} \mathrm{m}^{-1}$ are shown in the same brown color. Trace and retrace of each FD-curve are superimposed indicating no loss of energy, i.e. hysteresis, as shown in (D).

support, resulting in a FD-curve with an infinite slope (black curve). Pores which are not spanned by a membrane (e.g. due to membrane rupturing) also show a response curve with an infinite slope (blue curve) starting roughly $1 \mu \mathrm{m}$ below the pore rim. The offset between contact points can be explained by the fact that the cantilever tip moves into the uncovered pore until it gets into contact with the pore rim due to its pyramidal shape (see Fig. 3.21) in Chapter 3.3.2). FD-curves obtained on pore-spanning membranes (red curve) are linear with a finite slope and a contact point, which is located near the pore rim. From the slope $(F / h)$ of the curve, the apparent spring constant $k_{\text {app }}$ can be obtained, which reflects the overall response of the membrane upon indentation. The observation that trace and retrace of the 
FD curves are superimposed, indicates a fully elastic behavior of the membrane upon indentation (Fig. 4.5D). The small shift between the trace and the retrace can be attributed to hydrodynamic drag occuring during vertical movement of the cantilever. The distribution of $k_{\text {app }}$ within a force volume map was visualized by a $k_{\text {app }}$-map (Fig. 4.5D). The slope from each FD curve depicted in the force volume map in Figure 4.5A was determined and plotted for the corresponding position of the individual FD curve with a color code. The values were thresholded at $10 \mathrm{mN} \mathrm{m}^{-1}$ to exclude physical meaningless values obtained from FD curves recorded on the rim and on uncovered pores. The $k_{\text {app }}$-map reveals that $k_{\text {app }}$ values are consistent within the center of each f-PSM as observable by the same blue color coding. Yet, FD curves close to the pore rim show slight deviations from the values obtained in the pore center, which can be attributed to interactions between the pyramidal cantilever and the pore rim resulting in distorted FD curves with incorrect $k_{\text {app }}$ values. To ensure that exclusively undistorted FD-curves are considered for further investigations and to average over each PSM appropriately, 2x2 FD curves located in the center of each PSM were analyzed.

DPhPC PSMs on 6-MH/CPEO3 SAMs prepared from binary solutions containing $0 \mathrm{~mol} \%, 4.8 \mathrm{~mol} \%, 7.1 \mathrm{~mol} \%$ and $9.1 \mathrm{~mol} \%$ CPEO3 were analyzed by force indentation experiments. Figure 4.6A depicts typical FD curves obtained on the different SAMs. One finds, that all FD curves are linear over indentation depths of several ten to hundred nanometers. While PSMs on SAMs assembled from pure 6-MH $(0 \mathrm{~mol} \%$ CPEO3) exhibit FD curves with shallow slopes, PSMs on SAMs containing CPEO3 show curves with significantly steeper slopes. At least 3 different preparations per SAM composition were analyzed yielding a total of $1860 k_{\text {app }}$ values from 465 different PSMs. Steltenkamp and Mey et al. provided the first force indentation experiments on PSMs and could show that the lateral membrane tension $\sigma$ is the major contribution to $k_{\text {app }}$, while bending and stretching of the membrane can be neglected [62, 63]. Based on these findings, Norouzi et al. derived a theoretical framework to calculate the lateral membrane tension from the experimental $k_{\text {app }}$ values [84]:

$$
h=\frac{F}{4 \pi \sigma}\left[1-\ln \left(\frac{F \cdot R_{\mathrm{tip}}}{2 \pi \sigma\left(R_{\text {pore }}\right)^{2}}\right)\right] .
$$

With $F$ the force, $R_{\text {tip }}$ the cantilever tip radius and $R_{\text {pore }}$ the pore radius. Equation 4.1 was numerically solved with Mathematica (Wolfram Software, Oxfordshire, UK) for indentation depths of $30 \mathrm{~nm}$ assuming an indenter radius of $20 \mathrm{~nm}$ and a pore 
radius of $600 \mathrm{~nm}$ to convert the experimental $k_{\text {app }}$ into $\sigma$ (Fig. 4.6B). One finds, that increasing amounts of CPEO3 in the SAM leads to significantly higher lateral membrane tensions. While a PSM on a pure 6-MH SAM has a mean lateral membrane tensions of $1.4 \mathrm{mN} \mathrm{m}^{-1}$, a PSM on a SAM assembled from a $6-\mathrm{MH} / \mathrm{CPEO} 3$ solution with $9.1 \mathrm{~mol} \%$ CPEO3 displayed a lateral tension of $4.5 \mathrm{mN} \mathrm{m}^{-1}$, which is three times higher. PSMs on SAMs assembled from solutions containing $4.8 \mathrm{~mol} \%$ and $7.1 \mathrm{~mol} \%$ CPEO3, showed mean lateral tensions of $2.3 \mathrm{mN} \mathrm{m}^{-1}$ and $3.4 \mathrm{mN} \mathrm{m}^{-1}$, respectively. These results clearly demonstrate, that the chemical functionalization on the pore rim influences the lateral membrane tension in the f-PSM.

The lateral membrane tension of PSMs on binary 6-MH/CPEO3 SAMs was successfully measured by force indentation experiments. It could be verified that higher amounts of CPEO3 in the SAM significantly increase the lateral membrane tension of the f-PSM. PSMs on SAMs with high amounts of CPEO3 had a drastically reduced stability due to the high lateral tension. 

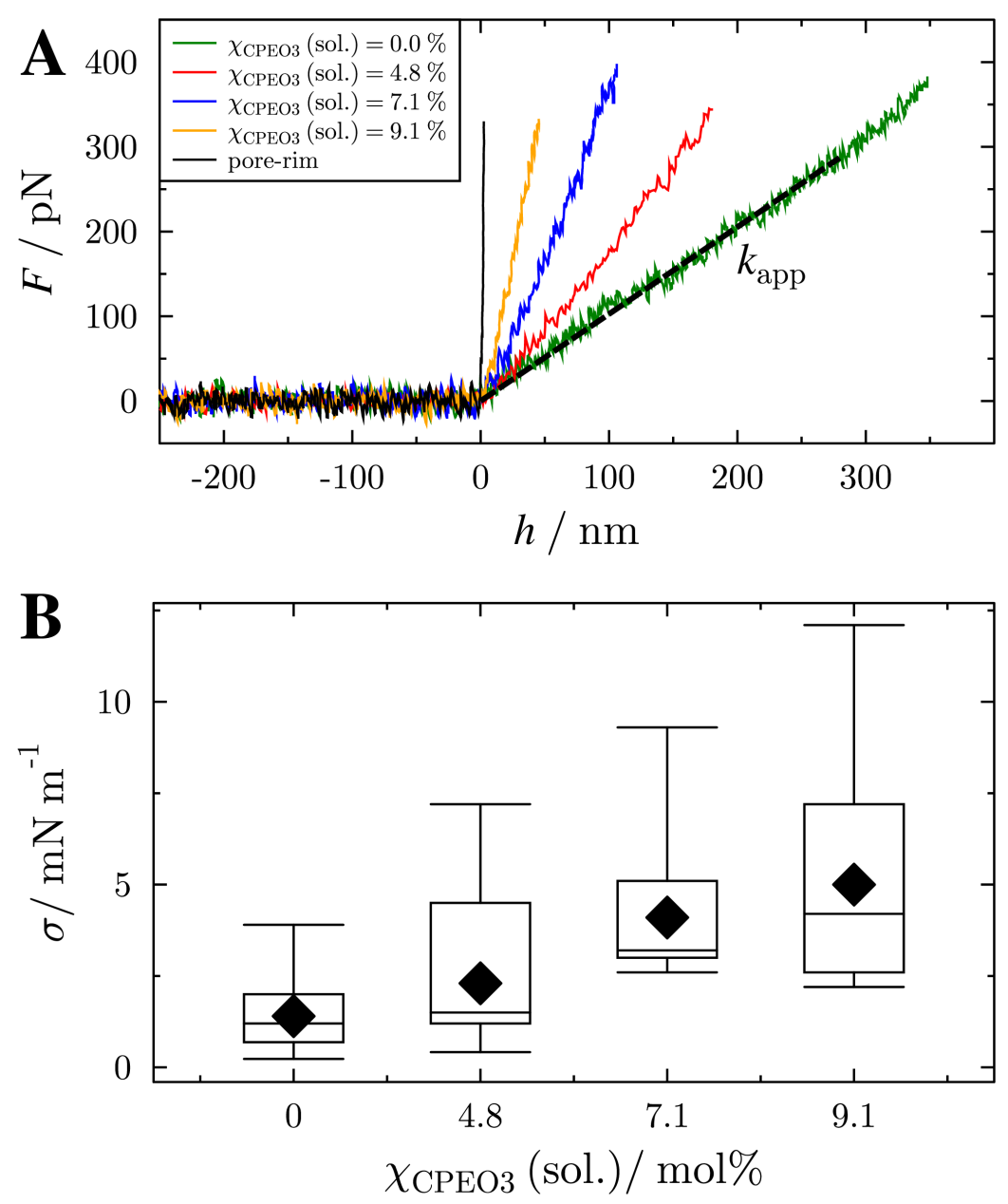

Figure 4.6. (A) Typical FD curves observed on a pore rim and on a pore-spanning membrane on SAMs composed of 6-MH and CPEO3. From the linear force response upon indentation the apparent spring constant $\left(k_{\text {app }}\right)$ of the system can be extracted. (B) Box plot diagram showing the lateral membrane tension derived from 404, 216, 732 and 520 FD curves from PSM on 6-MH/CPEO3 SAMs with 0, 4.8, 7.1 and $9.1 \mathrm{~mol} \%$ CPEO3 in the SAM solution, respectively. The ends of the whiskers mark the minimum and maximum values and the black diamonds the mean values. - is the median. 



\section{Discussion: Lateral membrane tension of pore-spanning membranes}

Pore-spanning membranes (PSMs) were prepared on gold coated porous substrates with pore diameters of $1.2 \mu \mathrm{m}$ functionalized with binary 6-MH/CPEO3 self-assembled monolayers and their mechanical properties were investigated by means of atomic force microscopy (AFM). With this approach, the influence of the surface functionalization on the lateral membrane tension was studied in more detail to pave the way for a model membrane system that allows to regulate tension. In a first step, the binary SAM was investigated by contact angle measurements and polarization modulation infra red reflection absorption spectroscopy (PM-IRRAS) to ascertain its proper assembly and to identify reasonable thiol ratios. In a second step, DPhPC membranes were prepared on these SAMs and analyzed by force spectroscopy to determine their lateral membrane tension.

\subsection{Formation of binary 6-MH/CPEO3 self-assembled monolayer}

Binary SAMs composed of the thiols 6-MH and CPEO3 were assembled from mixed solutions on gold surfaces and analyzed by contact angle and PM-IRRAS measurements. On pure 6-MH SAMs a mean contact angle of $19^{\circ}$ was measured, indicating a hydrophilic surface caused by the polar hydroxy groups. SAMs composed of pure CPEO3 resulted in a mean contact angle of $107^{\circ}$ due to the hydrophobic cholesterol residue. Both contact angles are in good agreement with values obtained on hydroxy $\left(16.9^{\circ}\right)$ and alkyl $\left(107.2^{\circ}\right)$ terminated SAMs proving successful SAM formation [85]. Mixed 6-MH/CPEO3 SAMs resulted in contact angles between those of the pure 
compounds. When the mole fraction of CPEO3 in solution was increased, higher contact angles could be observed, which is indicative of increasing amounts of CPEO3 in the SAM. This clearly proves that the ratio of the thiols in solution directly affect the SAM composition. Interestingly, rather low mole fractions of CPEO3 in the binary solution already resulted in drastically increased contact angles suggesting a faster adsorption kinetics in case of CPEO3. This hypothesis is supported by studies in which the preparation of mixed monolayers of alkyltrichlorosilanes with different chain lengths was investigated. Here, the SAM composition was also mainly determined by the relative adsorption rates of the single components [82]. If more than $15 \mathrm{~mol} \%$ CPEO3 were used in solution, no changes of the contact angle could be observed anymore indicating a saturation of the SAM with CPEO3. This phenomenon can be explained by the different packing densities of 6-MH molecules compared to that of CPEO3 on a gold surface. Alkylthiol molecules form a hexagonal $(\sqrt{3} \cdot \sqrt{3}) R 30^{\circ}$ structure on a gold [111] surface with a unit cell lattice spacing of $5 \AA$ providing a maximum cross sectional area of $21.4 \AA^{2}$ per molecule [86 89]. 6-MH molecules have a cross sectional area of approximately $17 \AA^{2}$ and can therefore occupy all available binding sites on the gold surface [79]. CPEO3 in contrast, has an approximate cross sectional area of $37 \AA^{2}$ permitting all binding sites to be filled [90, 91]. Considering that the cholesterol headgroups are densely packed and orientated perpendicular to the surface, one can derive a maximum occupancy of $58 \%$ compared to 6-MH. A SAM composed of only CPEO3 would therefore exhibit the same wettability as that composed of $58 \%$ CPEO3 and $42 \%$ 6- $\mathrm{MH}$. As the contact angle increases nearly linearly with the amount of CPEO3 in solution (see Fig. 4.2 in Chapter 4.1), one can estimate the amount of CPEO3 in the SAM. A conversion factor of 3.8 can be derived from the slope of the contact angle graph between the mole fraction of CPEO3 in a $n$-propanolic $6-\mathrm{MH} / \mathrm{CPEO} 3$ solution $\left(\chi_{\mathrm{CPEO} 3}(\mathrm{sol}).\right)$ and the resulting mole fraction of $\mathrm{CPEO} 3$ in the $\mathrm{SAM}\left(\chi_{\mathrm{CPEO} 3}(\mathrm{SAM})\right)$ :

$$
\chi_{\mathrm{CPEO} 3}(\mathrm{SAM})=3.8 \cdot \chi_{\mathrm{CPEO} 3}(\mathrm{sol} .) .
$$

Equation 5.1 yields only correct results for $\chi_{\mathrm{CPEO}(\mathrm{sol} .} \leq 15 \mathrm{~mol} \%$. The binary 6 $\mathrm{MH} / \mathrm{CPEO} 3$ SAMs were further analyzed by PM-IRRAS to obtain chemical information, which supports the contact angle measurements. Characteristic absorption signals from the 6-MH and the CPEO3 SAMs could be observed proving successful SAM formation without significant impurities. To analyze changes in the binary SAMs, the height of the $\mathrm{C}=\mathrm{O}$ absorptions and was plotted as a function of the mole fraction 
of CPEO3 in the solution. A maximum absorption peak could be observed for SAMs assembled from solutions containing more than 20 mol\% CPEO3 indicating a CPEO3 saturation of the SAM. This observation is in good agreement with the contact angle measurements, where $15 \mathrm{~mol} \%$ CPEO3 were sufficient to saturate the SAM.

\subsection{Lateral membrane tension of pore-spanning membranes on 6-MH/CPEO3 self-assembled monolayers}

PSMs on differently composed binary 6-MH/CPEO3 SAMs were anaylzed by AFM to investigate the influence of the SAM on the lateral membrane tension of the PSM. Steltenkamp and Mey et al. were the first who investigated the mechanical properties of PSMs by force indentation experiments. They found that PSMs show a linear force response upon indentation, which is mainly governed by prestress in the membrane, called lateral membrane tension [62]. The prestress in the membrane originates from the free energy difference between the freestanding and the adhered membrane on the support. Based on these studies, Norouzi et al. developed a theoretical framework to calculate the lateral membrane tension from the experimental FD curves (ref). Mey et al. used hybride PSMs on hydrophobic octanethiol SAMs by either spreading GUVs to obtain solvent-free membranes or by painting lipids dissolved in $n$-decane to obtain solvent containing membranes (nano-BLMs). Due to the hydrophobic SAM, the resulting hybride PSMs exhibit a lipid monolayer on the pore rims, while the freestanding parts are composed of a continuous lipid bilayer (Fig. 5.1). The strong adhesion energy between the lipid hydrocarbon chains and the OT were explained to drastically increase the free energy difference between the supported and the freestanding membrane, leading to a lateral prestress in the freestanding membrane [62]. These membranes therefore resemble large lateral membrane tensions in the range of $20-30 \mathrm{mN} \mathrm{m}^{-1}$. The hybrid structure of the membrane also resulted in membrane rupturing during force indentation experiments, when area dilatations of more than $1 \%$ were exceeded.

Kocun et al. developed solvent free PSMs on pure hydrophilic 2-mercaptoethanol (2-ME) SAMs [5]. Here, the PSM consisted of a continuous lipid bilayer with a very thin, 1-2 nm thick water layer, located in the space between the SAM and the PSM (Fig. 5.2). Analysis of the mechanical properties of these membranes revealed lateral 


\section{A Hybride pore spanning membrane}

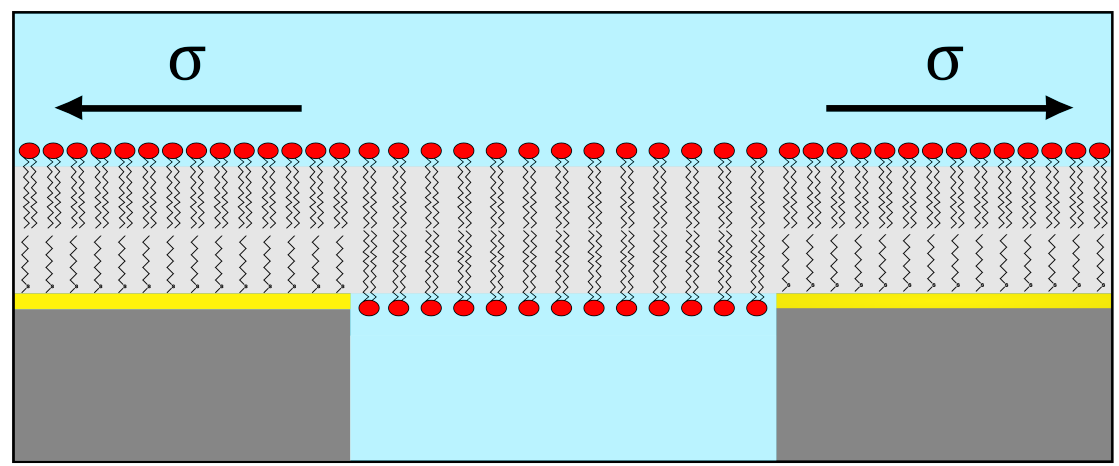

Figure 5.1. Illustration of a hybrid pore-spanning membrane on an octanethiol SAM. The hydrophobic SAM substitutes the lower leaflet of the lipid bilayer on the support. The large adhesion energy between the SAM and the lipids, results in a high lateral membrane tension $(\sigma)$ in the freestanding membrane as indicated by the long arrows.

membrane tensions in the range of $1-3 \mathrm{mN} \mathrm{m}^{-1}$, which are much lower than those obtained for hybrid PSMs. The authors explained these differences with a low adhesion energy between the SAM and the lipid bilayer, resulting in lower lateral tensions. In contrast to hybrid PSMs, rupturing of these membranes could only very rarely be observed even at indentation depths, which would result in membrane dilatations larger than $5 \%$. The authors inferred from these observations that the membrane flows from the pore rim into the pore upon indentation as membrane rupturing would already occur if area dilatation exceeds $3-5 \%$ [92, 93]. This interpretation is supported by recent studies from Gleisner et al. They protruded PSMs, spanned over sealed pores, by applying an osmotic gradient. During the growth of the protrusions, the PSM patch area decreased indicating that the membrane flows into the protruded structures [94].

In this work, PSMs were prepared on mixed 6-MH/CPEO3 SAMs containing different amounts of hydrophobic CPEO3 for the first time. The SAMs were prepared from $n$-propanolic 6-MH/CPEO3 solutions containing 0, 4.8, 7.1 and 9.1 mol\% CPEO3, corresponding to 0, 18, 27 and $35 \mathrm{~mol} \%$ CPEO3 in the SAM, as these mixtures allowed to vary the SAM composition over a wide range (see Chapter 5.1) and display sufficient stabilities (see Chapter 4.3). The lateral tension of the PSMs were systematically investigated by force indentation experiments as a function of the amount of CPEO3 in the SAM. PSMs on pure hydrophilic 6-MH SAMs exhibited the lowest membrane tensions with a mean value of $\sigma=1.4 \mathrm{mN} \mathrm{m}^{-1}$. These results are 


\section{B Pore spanning membrane}

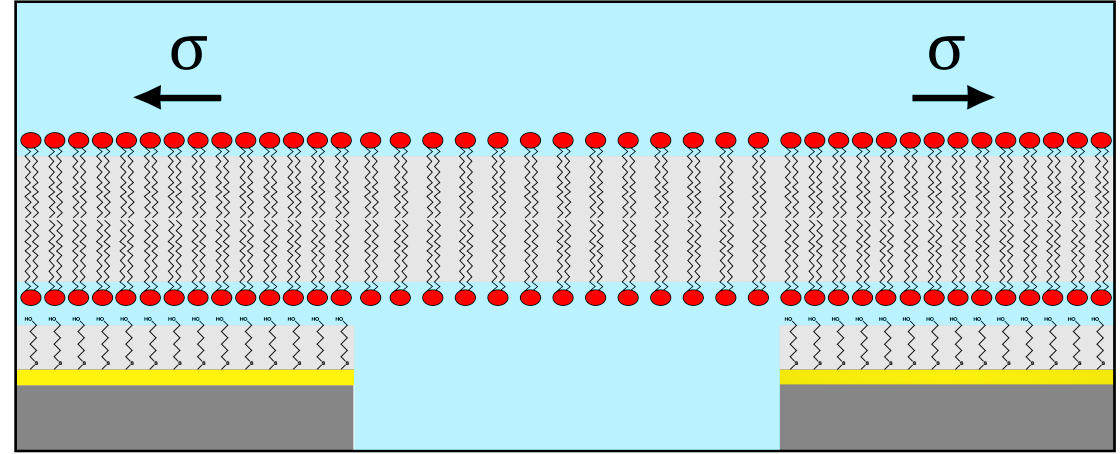

Figure 5.2. Illustration of a pore-spanning membrane on a 2-mercaptoethanol SAM. The hydrophilic SAM supports an continuous lipid bilayer on the substrate. The adhesion energy between the lipid headgroups and the SAM is significantly lower than the hydrophobic interactions in hybrid PSMs, which results in much lower lateral membrane tensions.

similar to those obtained for DOPC $\left(1.08 \mathrm{mN} \mathrm{m}^{-1}\right)$ and POPC $\left(2.0 \mathrm{mN} \mathrm{m}^{-1}\right)$ PSMs on 2-mercaptoethanol SAMs determined by Kocun et al. [5]. These observations are reasonable, as 6-MH and 2-ME SAMs exhibit similar hydrophilicities. Only the utilized lipids (DPhPC vs. DOPC and POPC) differ, which could explain the slight deviations between the measured tensions. The very similar tensions also underline, that the synthetic lipid DPhPC yields results, which are comparable to those of PSMs composed of more physiological lipids like DOPC and POPC. PSMs on binary 6-MH/CPEO3 SAMs showed higher lateral membrane tensions than PSMs on pure 6-MH. For PSMs on 6-MH/CPEO3 SAMs containing 18, 27 and 35 mol\% CPEO3, mean lateral tensions of $2.3,3.4$ and $4.5 \mathrm{mN} \mathrm{m}^{-1}$ were observed, respectively. This result indicates that higher amounts of CPEO3 in the SAM lead to higher lateral membrane tensions in the PSMs. This can be explained by the structure of the 6-MH/CPEO3 SAM interacting with the lipid bilayer. The hydrophobic cholesterol residue of the CPEO3 molecules tower over the hydrophilic 6-MH SAM and insert into the hydrophobic core of the bilayer (Fig. 5.3) [79]. The interaction between the cholesterol group and the hydrocarbon chains of the lipids leads to a high local adhesion energy comparable to hybrid PSMs explaining the larger lateral tensions. With larger amounts of CPEO3 in the SAM, indicated by larger contact angles, more hydrophobic insertion sites for the bilayer are available.

It was found that the stability of the freestanding DPhPC membranes drastically decreased with higher amounts of CPEO3 in the SAM (see Chapter 4.3). This observation 
can be explained with the higher lateral tensions in the membrane leading to spontaneous rupturing. PSMs composed of more natural lipid compositions (POPC/cholesterol (8:2) and (DOPC, POPS, POPE, cholesterol (5:1:2:2)) were found to be even more instable. The greater instability of these PSMs in comparison to those composed of $\mathrm{DPhPC}$ is most probably a direct result of the different area compressibility moduli of the lipids, which limit the maximal applicable lateral membrane tension. While DPhPC has an area compressibility modulus of $670 \mathrm{mN} \mathrm{m}^{-1}$, typical fluid phase lipids like POPC and DOPC have area compressibility moduli of only $200-250 \mathrm{mN} \mathrm{m}^{-1}$ and are thus more prone to rupture under high membrane tensions [61].

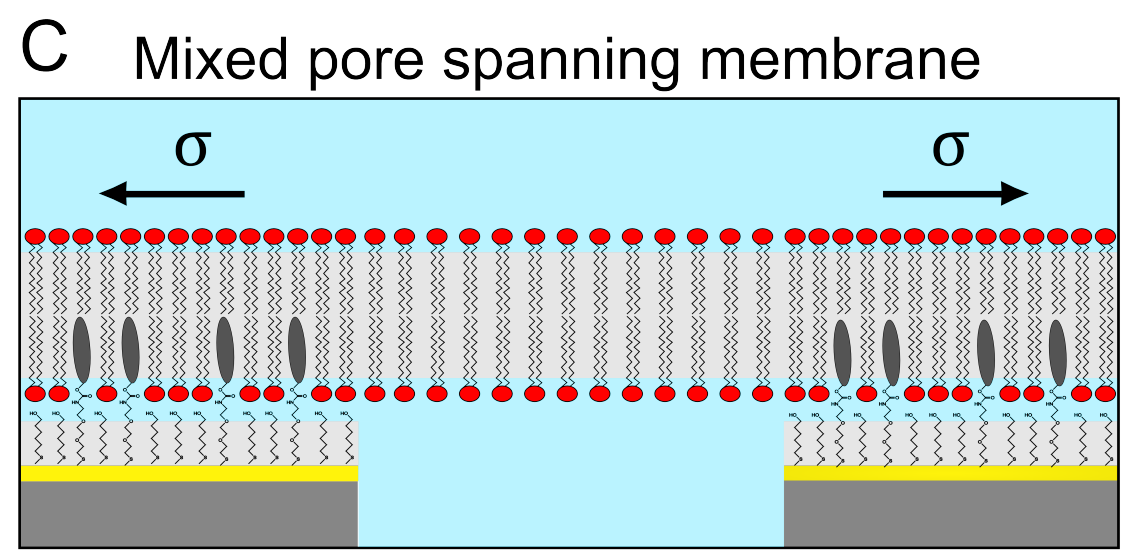

Figure 5.3. Illustration of a pore-spanning membrane on a binary 6-MH/CPEO3 SAM. The hydrophilic 6-MH SAM supports and decouples the lipid bilayer from the substrate, while the cholesterol residue (black) of CPEO3 inserts into the hydrophobic core of the bilayer. The cholesterol groups locally result in a higher adhesion energy, increasing the lateral tension of the PSM.

SAMs composed of 6-MH and CPEO3 were successfully investigated and utilized to manipulate the lateral membrane tension of PSMs. The mean lateral tension of DPhPC PSMs could be modulated between $1.4-4.5 \mathrm{mN} \mathrm{m}^{-1}$ using increasing amounts of CPEO3 in a binary SAM composed of 6-MH and CPEO3, offering the possibility to directly influence the membrane tension by changing the composition of the SAM. The obtained lateral tensions were non-physiological, as they are significantly higher than those obtained in cell membranes $\left(0.003-0.45 \mathrm{mN} \mathrm{m}^{-1}\right)$ [6 9]. The high lateral tensions on the binary SAMs also effected the stability of the freestanding membranes, making it necessary to use very stable lipids with a high area compression modulus like DPhPC. Therefore processes, which require natural lipid compositions, like SNARE-mediated membrane fusion (see Chapter 6.2), cannot be studied in this system. 


\section{Results: SNARE-mediated fusion on pore-spanning membranes}

Fusion of two distinct lipid bilayers driven by SNAREs is a fundamental process taking place at the nerve terminal in eukaryotic cells prior to neurotransmitter release. In order to study the mechanism and kinetic of this prominent process in a well defined manner, SNAREs have been reconstituted into artificial membranes serving as in-vitro fusion systems [24, 32, 46, 95, 97]. Yet, many of those assays monitor fusion in bulk experiments averaging over a large vesicle ensemble. In order to study fusion on a single vesicle level, which allows to distinguish between docking and fusion and enables one to identify fusion intermediates, planar solid supported target-membranes were developed [45, 48 51, 53, 54, 98, 99]. PSMs are accessible by various fluorescence microscopy techniques and mimic the planar pore geometry of the synapse. A major disadvantage of these system is their entirely supported membrane, which does not offer a second aqueous volume under the membrane. To overcome this disadvantage pore-spanning membranes (PSMs) were recently developed as a advanced model system by Höfer and Schwenen et al. [55, 56]. PSMs are prepared by spreading giant unilamellar vesicles (GUVs) over a porous support that is comprised of evenly arranged cylindrical pores with pore diameters ranging from $1.2-5.0 \mu \mathrm{m}$ resulting in membrane patches that consist of an entire fully mobile lipid bilayer [5, 56]. Some part of the membrane patch is supported by the surface surrounding the pores, called supported-Pore Spanning Membrane (s-PSM) while the residual membrane traverses the pores of the substrate, called free-standing-Pore Spanning Membrane (f-PSM) facing an aqueous reservoir on both sides.

In this work SNARE-mediated fusion on planar pore-spanning membranes was further investigated using high speed spinning disc fluorescence microscopy and porous substrates with large $5 \mu \mathrm{m}$ pores to get a detailed insight into the kinetics of SNARE fusion 
on planar supported and free-standing target-membranes. In a first step, SNARE reconstitution into small vesicles and GUVs, used to prepare PSMS, was quantified and their functionality verified by bulk SNARE activity experiments. In a second step, docking and fusion of small and large syb 2 vesicles on supported and free-standing PSMs containing the t-SNARE $\Delta \mathrm{N}$-complex was investigated on a single vesicle level with $10 \mathrm{~ms}$ time resolution. Parts of this work have been published in the Biophysical Journal [100].

\subsection{Reconstitution of SNAREs into liposomes and pore-spanning membranes}

A minimal fusion machinery comprised of the target-SNARE (t-SNARE) $\Delta$ N-complex and the vesicular-SNARE (v-SNARE) synaptobrevin 2 (syb2) was used as these proteins resemble the central fusion motif and are sufficient to initiate fusion [28]. The $\Delta \mathrm{N}$-complex consists of syntaxin $1 \mathrm{~A}$, here used in a truncated version lacking the regulatory $\mathrm{H}_{\mathrm{abc}}$ domain (residues 183-288), SNAP 25a and a syb 2 fragment (residues 4996) that stabilizes the complex and prevents the spontaneous formation of an inactive syntaxin 1A/SNAP 25a 2:1 complex [28, 49]. The $\Delta$ N-complex was reconstituted into GUVs, which were then spread on porous substrates to prepare t-SNARE containing PSMs while full length syb 2 was reconstituted into vesicles with diameters of $90 \mathrm{~nm}$ and $240 \mathrm{~nm}$. A protocol initially developed by Pobbati et al. and further revised by Schwenen et al. for PSMs was used for SNARE reconstitution [28, 56]. The protocol is based on the co-micellization of proteins and lipids and a subsequent rapid detergent removal via size exclusion chromatography yielding small proteo-liposomes. The small proteo-liposomes exhibit diameters of $50-100 \mathrm{~nm}$ and provide the basis to prepare proteo-liposomes with larger diameters of $100-400 \mathrm{~nm}$ and proteo-GUVs with diameters of $10-100 \mu \mathrm{m}$ (see Chapter 3.2.3).

To verify the successful incorporation of SNAREs into liposomes and GUVs, each reconstitution step was analyzed in detail. In all experiments an initial molar protein to lipid (p:l) ratio of 1:500 was used if not mentioned otherwise to approximately match the number of SNAREs in plasma membrane of secretory cells [101]. A lipid mixture of DOPC/POPE/POPS/cholesterol (5:2:1:2) was used throughout the experiments as this mixture resembles the composition of the plasma membranes very well and was used recently for fusion experiments on PSMs [56]. In a first step, the incorporation of syb 2 and $\Delta \mathrm{N}$-complex into liposomes directly obtained from the size exclusion 
column was analyzed as these vesicles resemble the starting material for all further reconstitution steps into LUVs and GUVs. The proteo-liposomes were submitted to a nycodenz floatation assay to separate proteo-liposomes from unreconstituted protein by a density gradient (see Chapter 3.2.3). Upon centrifugation a nycodenz density gradient originates in the centrifuge tube in which buffer-filled SUVs initially located at the bottom float to the less dense top, while unreconstituted proteins remain on the much denser bottom of the gradient. Fractions, carefully collected from top to bottom of the gradient after centrifugation, were subsequently analyzed by SDS-PAGE. Figure 6.1 depicts SDS-PAGE gels of syb 2 liposomes (A) and $\Delta \mathrm{N}$-complex liposomes (B). Characteristic bands of syb 2 at $14 \mathrm{kDa}$ and of the $\Delta \mathrm{N}$-complex that disassembles in the proteins syx 1A, SNAP 25a and syb 2 (residues 49-96) at 23, 12 and $5 \mathrm{kDa}$ in the SDS-PAGE gel occur mainly in the top fractions of the gradients indicating a successful incorporation of the proteins into the liposomes. No protein bands can be observed in the bottom fractions indicating an excellent reconstitution efficiency of over $90 \%$ for the $\Delta \mathrm{N}$-complex and syb 2 . Syb 2 LUVs obtained after the second reconstitution protocol step and extrusion through a $400 \mathrm{~nm}$ polycarbonate membrane were analyzed according to the proteo-liposomes from the size exclusion column by a nycodenz floatation assay. Figure 6.1 C shows the SDS-PAGE gel obtained after fractionating the gradient on the gel. Again, syb 2 is located mainly in the top fractions of the gradient indicating a successful reconstitution into LUVs. The protein and lipid concentration is much lower after the second reconstitution step as observable by the less intense protein bands in the SDS-PAGE gel. This is caused by a loss of proteins and lipids during the drying and extrusion process. To quantify the overall loss of lipids and proteins during the whole reconstitution, a quantitative phosphate test and SDS-PAGE was performed. Only $13 \%$ of the initially used lipids can be recovered in the LUVs. Roughly the same percentage $(10 \%)$ of syb 2 remains in the LUVs, confirming that the initial lipid to protein ratio remains nearly the same. Taking these results into account, the average number of syb 2 molecules per vesicle can be calculated to 160 syb 2/vesicle, assuming a vesicle diameter of $100 \mathrm{~nm}$ and a mean lipid area of $0.65 \mathrm{~nm}^{2}$. The reconstitution efficiency of $\Delta \mathrm{N}$-complex into GUVs could not be analyzed via a floatation assay as it was performed for LUVs due to the following two reasons. First, GUVs are prepared in sucrose solution, which has a significantly higher density than the reconstitution buffer of the LUVs. Therefore GUVs would not float to the very top of the nycodenz gradient and partly mix up with fractions carrying unreconstituted protein. Second, GUV suspensions commonly 
obtained after electroformation are highly contaminated with membrane debris making it necessary to separate intact GUVs from the debris. Although a strategy could be developed during this work to purify the GUVs by sedimentation in less dense glucose solutions or buffer, the final amounts of GUVs obtained after this procedure were not sufficient for the subsequent SDS-PAGE analysis. Therefore, a fluorescently labeled $\Delta \mathrm{N}$-complex, (Atto488- $\Delta \mathrm{N}$-complex) was synthesized (see Chapter 3.2.2) and reconstituted according to the unlabeled $\Delta \mathrm{N}$-complex into GUVs and analyzed by fluorescence microscopy. To obtain enough fluorescence signal from the labeled GUVs, the initial p:l ratio was increased from 1:500 to 1:125. Figure 6.1 D depicts a fluorescent micrograph of Atto488- $\Delta \mathrm{N}$-complex GUVs. A homogeneous but rather weak fluorescence signal can be observed of the GUVs demonstrating the successful incorporation of the Atto488- $\Delta \mathrm{N}$-complex into the GUV membrane. Also very bright aggregates arising from labeled $\Delta \mathrm{N}$-complex were found in the suspensions, giving rise to significant amounts of unreconstituted protein. To roughly estimate the final p:1 ratio in the proteo-GUVs, GUVs that were labeled with different concentrations of Atto488-DPPE ranging from $0.001-0.1 \mathrm{~mol} \%$ were imaged with the same parameters by fluorescence microscopy and the intensities compared with those of the proteoGUVs. Although this method does not yield precise quantitative data, it nicely allows to estimate the p:l ratio. It could be estimated that less than $1 \%$ of the initially introduced $\Delta \mathrm{N}$-complex is actually incorporated into the GUVs. This translates into $120 \Delta \mathrm{N}$-complexes per $\mu \mathrm{m}^{2}$ in the GUVs assuming an average lipid area of $0.65 \mathrm{~nm}^{2}$. The successful reconstitution of the SNAREs syb 2 and $\Delta$ N-complex into LUVs and GUVs could be verified. Syb2 LUVs exhibit a final l:p ratio 1:600 indicating an excellent reconstitution efficiency. The $\Delta \mathrm{N}$-complex GUVs exhibit an estimated p:1 ratio of 1:50000, which corresponds to an reconstitution efficiency of $1 \%$. 

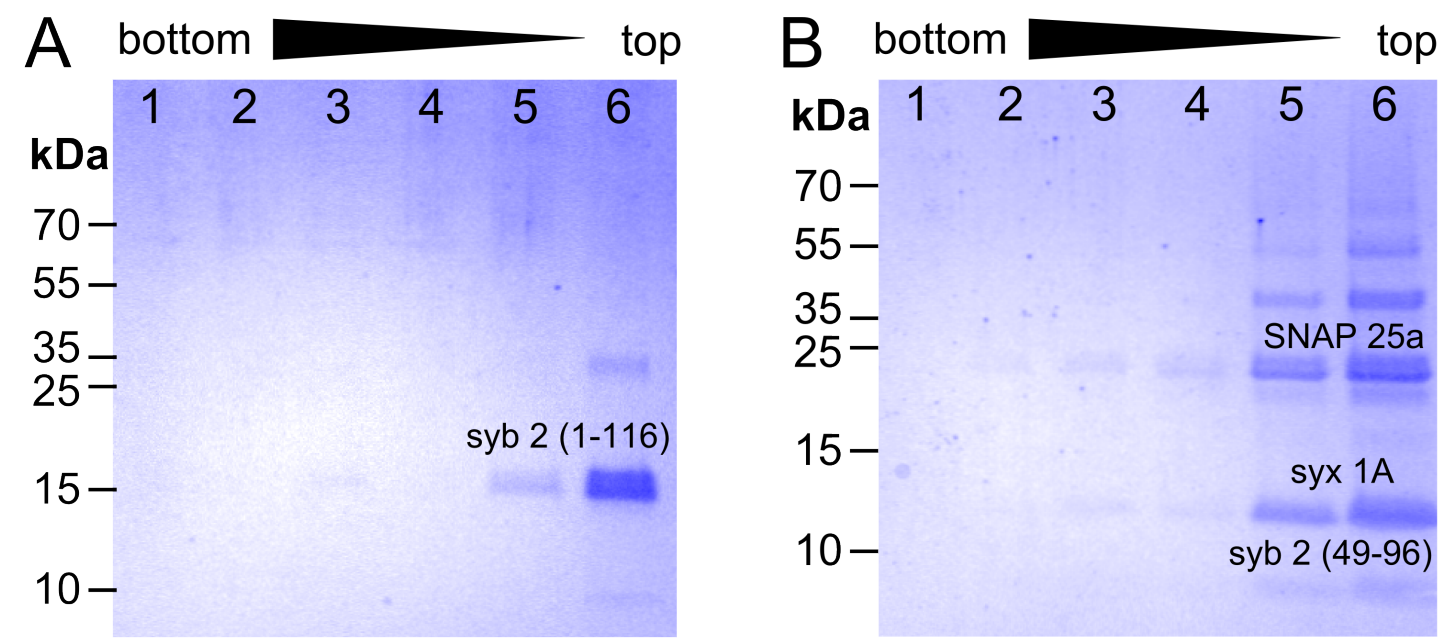

\section{C bottom}

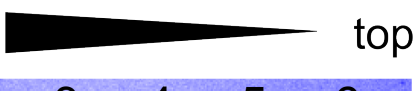

D
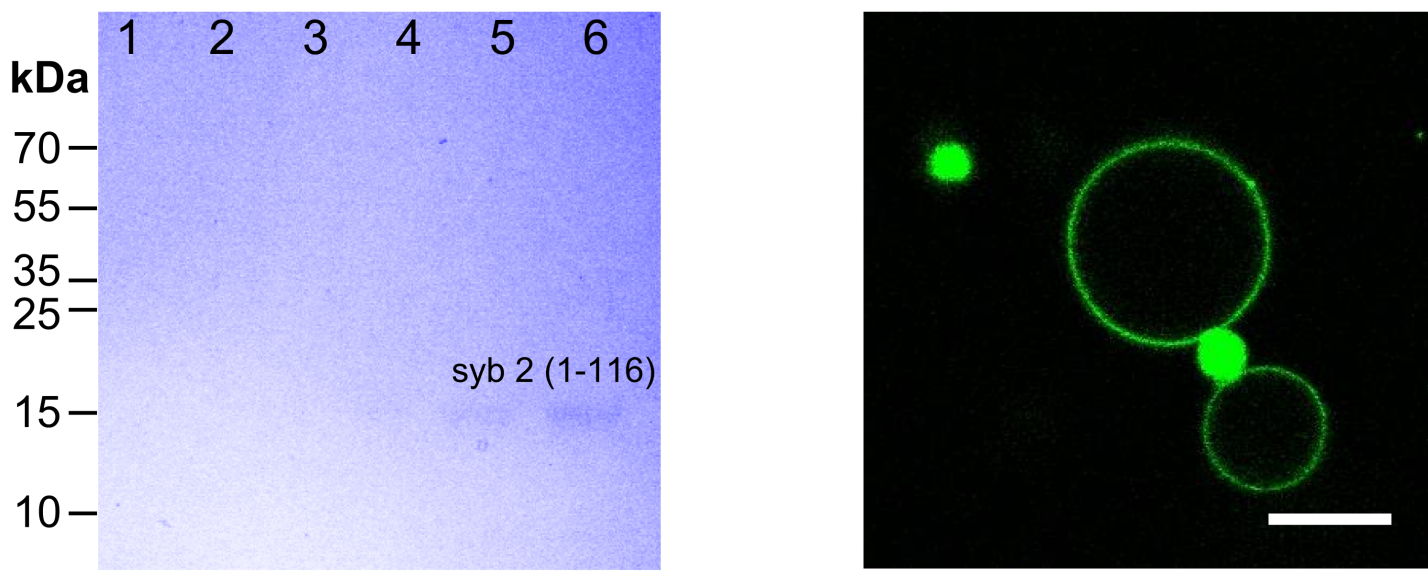

Figure 6.1. SDS-PAGE gel of fractions obtained from a nycodenz floatation assay performed with syb 2 SUVs (A) and $\Delta \mathrm{N}$ SUVs (B) as well as with syb 2 LUVs (C) composed of DOPC/POPE/POPS/cholesterol (5:2:1:2). Reconstituted protein co-migrates with the liposomes to the top of the gradient while free protein is located at the bottom. (D) Fluorescence micrograph of GUVs composed of DOPC/POPE/POPS/cholesterol (5:2:1:2) obtained after reconstitution of fluorescently labeled Atto488- $\Delta \mathrm{N}$-complex. Scale bar: $12 \mu \mathrm{m}$. The initial p:l ratio was fixed to 1:500 for the density gradient experiments and to 1:125 for the fluorescence based assay. 


\subsection{SNARE-activity in liposomes}

The functionality of reconstituted SNAREs in vesicles composed of DOPC/POPE/POPS/chol (5:1:2:2) was analyzed by bulk activity experiments in a spectrofluorimeter by mixing $\Delta \mathrm{N}$-complex vesicles doped with $1 \mathrm{~mol} \%$ Atto488-DPPE (Atto488) and syb 2 vesicles doped with 1 mol \% TexasRed-DHPE (TR). The mixed vesicle suspension was illuminated at $488 \mathrm{~nm}$ in a glass cuvette to excite the Atto488 doped vesicles while the emission at $608 \mathrm{~nm}$, depicting the emission maximum of TR, was recorded perpendicular to the excitation axis. Upon SNARE driven docking and fusion of vesicles, the donor Atto488 and the acceptor TR come into close proximity due to lipid mixing resulting in a Förster resonance energy transfer (FRET) leading to an increase in TR fluorescence, which is analyzed. SNARE activity between syb 2 and $\Delta \mathrm{N}$-complex containing liposomes, obtained directly after size exclusion chromatography, was first monitored via FRET as shown in Figure 6.2 A (blue curve). An increase in TR fluorescence intensity over time could be observed indicating a FRET and SNARE activity. To exclude unspecific fusion or binding that leads to an increase in TR intensity, control experiments where conducted, where the binding site for syb 2 of the $\Delta \mathrm{N}$-complex was irreversibly blocked with a water soluble syb 2 fragment (residues 1-96) prior to the experiments as shown in Figure 6.2A (red curve). No increase in TR fluorescence intensity over time could be observed in these experiments, proving SNARE specific activity. SNARE activity in syb 2 LUVs with a mean diameter of $240 \mathrm{~nm}$ and $\Delta \mathrm{N}-\mathrm{GUVs}$ was analyzed in the same manner (Fig. 6.2B) (orange curve). An increase in TR fluorescence intensity could be observed indicating SNARE activity between LUVs and GUVs. A slightly different kinetic can be observed, which can be attributed to the different sizes and concentrations of the vesicle populations. The specificity of SNAREs was also tested with a control experiment, where the t-SNARE binding motif of the $\Delta \mathrm{N}$-complex was blocked with the water soluble syb 2 fragment (residues 1-96) prior to the experiments as depicted in Figure $6.2 \mathrm{~B}$ (green curve). No activity was found in these experiments. SNARE activity was additionally tested in liposomes prepared from the synthetic lipid DPhPC to analyze, if such stable and non physiological lipids resemble suitable membranes for fusion. In case of small DPhPC vesicles containing SNAREs, fusion could be monitored in the activity tests. However fusion between DPhPC LUVs and GUVs could not be observed indicating that DPhPC does not resemble a suitable lipid to study SNARE-mediated fusion in GUVs or in systems that are prepared from GUVs, like PSMs. 
With the bulk SNARE activity assay, the functionality of reconstituted syb 2 and $\Delta \mathrm{N}$-complex could be verified in SUVs, LUVs and GUVs.
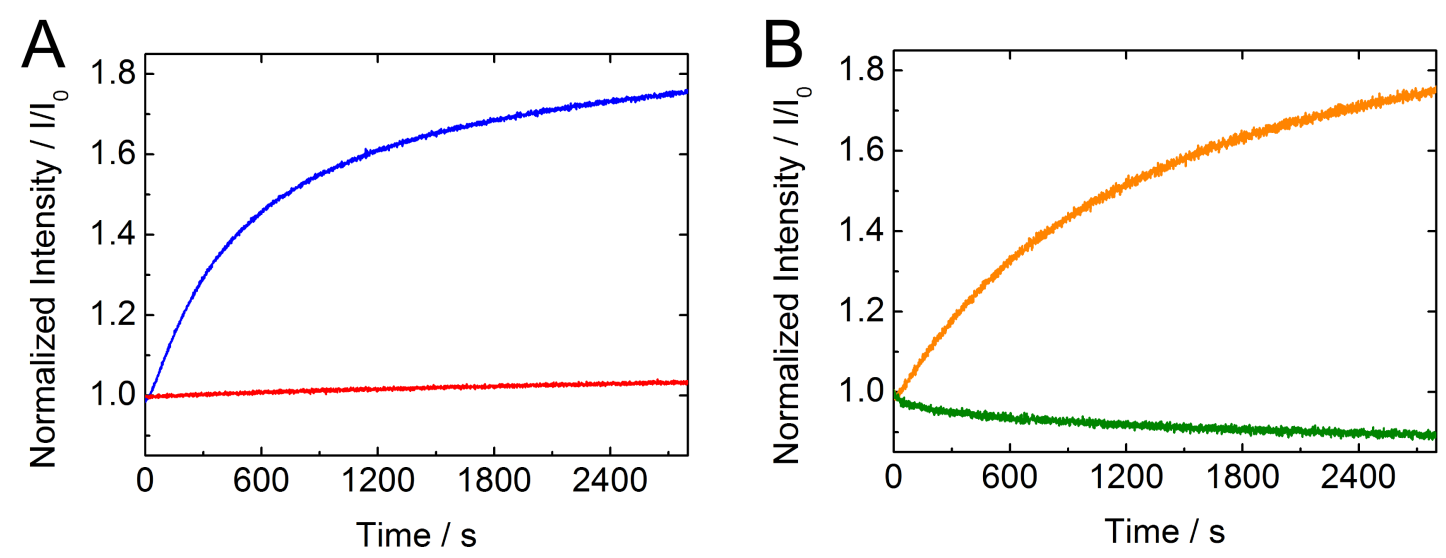

Figure 6.2. Intensity of $\mathrm{TR}$ acceptor fluorescence vs. time traces obtained in bulk activity assays. (A) Activity assay obtained from mixing syb 2 and $\Delta \mathrm{N}$-complex containing vesicles after size exclusion chromatography comprised of DOPC/POPE/POPS/cholesterol (5:2:1:2) doped with $1 \mathrm{~mol} \% \mathrm{TR}$ and Atto488, respectively (blue curve) and the corresponding control experiment with an irreversibly blocked $\Delta \mathrm{N}$-complex (red curve). (B) Activity assay obtained from mixing syb 2 vesicles obtained after extrusion through a $400 \mathrm{~nm}$ polycarbonate membrane and $\Delta \mathrm{N}$-complex GUVs comprised of DOPC/POPE/POPS/cholesterol (5:2:1:2) doped with $1 \mathrm{~mol} \%$ TR and Atto488, respectively (orange curve) and the corresponding control experiment with an irreversibly blocked $\Delta \mathrm{N}$-complex (green curve).

\subsection{Mobility of lipids and SNAREs in pore-spanning membranes}

The mobility of lipids and especially of the SNAREs in membranes is prerequisite for the successful assembly and fusion of two membranes, and was therefore analyzed in s-PSMs and f-PSMs. Schwenen et al. recently measured the mobility of fluorescently labeled lipids (Atto488-DPPE) and a fluorescently labeled syntaxin 1A-transmembrane domain (Atto647N-Syx 1A-TMD), resembling the part that anchors the $\Delta \mathrm{N}$-complex in the membrane, in f-PSMs composed of DOPC/POPE/POPS/cholesterol (5:2:1:2) spanning pores with a diameter of $1.2 \mu \mathrm{m}$ by fluorescence correlation spectroscopy (FCS) [56]. A mean diffusion coefficient of $7.7 \mu \mathrm{m}^{2} \mathrm{~s}^{-1}$ for Atto488-DPPE and $3.4 \mu \mathrm{m}^{2} \mathrm{~s}^{-1}$ for Atto647N-syx 1A-TMD were determined. The values are in good agreement with those found in free-standing membranes of GUVs [102, 103]. The reduced mobility of 
the Atto647N-syx 1A-TMD compared to Atto488-DPPE was explained by the larger molecular area of the transmembrane domain and the transmembrane helix spanning both leaflets of the bilayer. The mobilities of the membrane components in the s-PSMs could unfortunately not be measured by FCS as the gold layer on the pore rims quenches fluorescence signals very efficiently [5, 104, 105]. To overcome these problems, indirect fluorescence recovery after photobleaching (FRAP) was utilized in this work to gather information about the mobilities of lipids and proteins in the s-PSM. Indirect FRAP was conducted by bleaching a round region of interest (ROI), covering the main area of an f-PSM, and measuring the fluorescence recovery into the ROI as a function of time (Fig. 6.3). As the ROI is surrounded by s-PSM, the fluorescence material that recovers back into the ROI originates from the surrounding s-PSM and indirectly proves its mobility. Indirect FRAP measurements were performed on PSMs composed of DOPC/POPE/POPS/cholesterol (5:2:1:2) doped with either 1 mol \% Atto488-DPPE (Fig. 6.3A) or $1 \mathrm{~mol} \%$ OG-syx 1A-TMD (Fig. 6.3B). The transmembrane peptide OG-syx 1A-TMD was used for the experiments, as the Atto488- $\Delta \mathrm{N}$-complex yielded not enough fluorescence intensity for the FRAP experiments and to compare the results with those obtained by Schwenen et al. In all FRAP experiments a full recovery of the fluorescence intensity could be observed in the ROI after the bleach pulse with a mean halftime of $t_{\text {half }}=0.4 \mathrm{~s}$ for Atto488-DPPE and $t_{\text {half }}=1.2 \mathrm{~s}$ for OG-syx 1A-TMD, indicating a reasonable mobility of lipids and TMD-anchored proteins in the s-PSM. The lipid Atto488-DPPE recovers significantly faster than the OG-syx 1A-TMD, which could also be observed in the FCS measurements on the f-PSM.

The indirect FRAP experiments are well suited to prove the mobility of fluorescently labeled lipids and peptides in the s-PSM and to qualitatively compare the recovery curves. Yet, it is not possible to derive absolute diffusion coefficients from the recovery curves, as the recovery is a deconvolution of the diffusion in the s-PSM and in the f-PSM occupying $36 \%$ of the total PSM area around the ROI. To determine absolute diffusion coefficients from the indirect FRAP experiments, the experiments were simulated assuming different diffusion coefficients in the s-PSM and in the f-PSM by finite element simulations (COMSOL Multiphysics v4.4) with a 2D model solving Fick's second law [106]:

$$
\frac{\partial c_{i}}{\partial t}=D_{i} \nabla^{2} c_{i}
$$

with $t$ the time, $D$ the diffusion coefficient and $c_{i}$ the surface concentration. The hexagonal pore geometry of the PSMs was modeled on a square $60 \mathrm{x} 60 \mu^{2}$ area by 


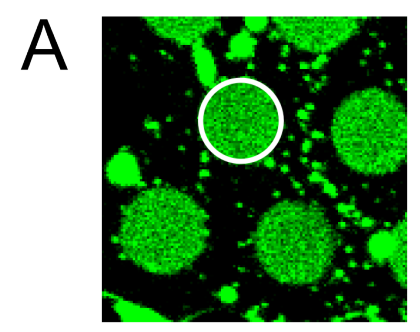

prebleach

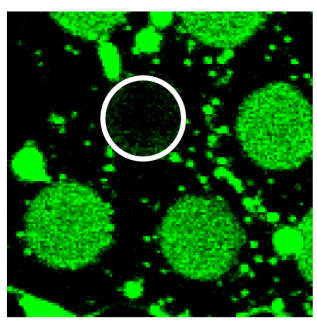

bleach

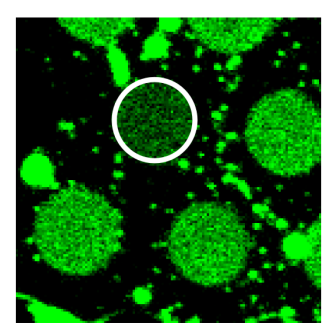

recovery

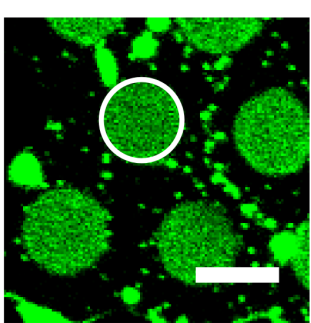

end
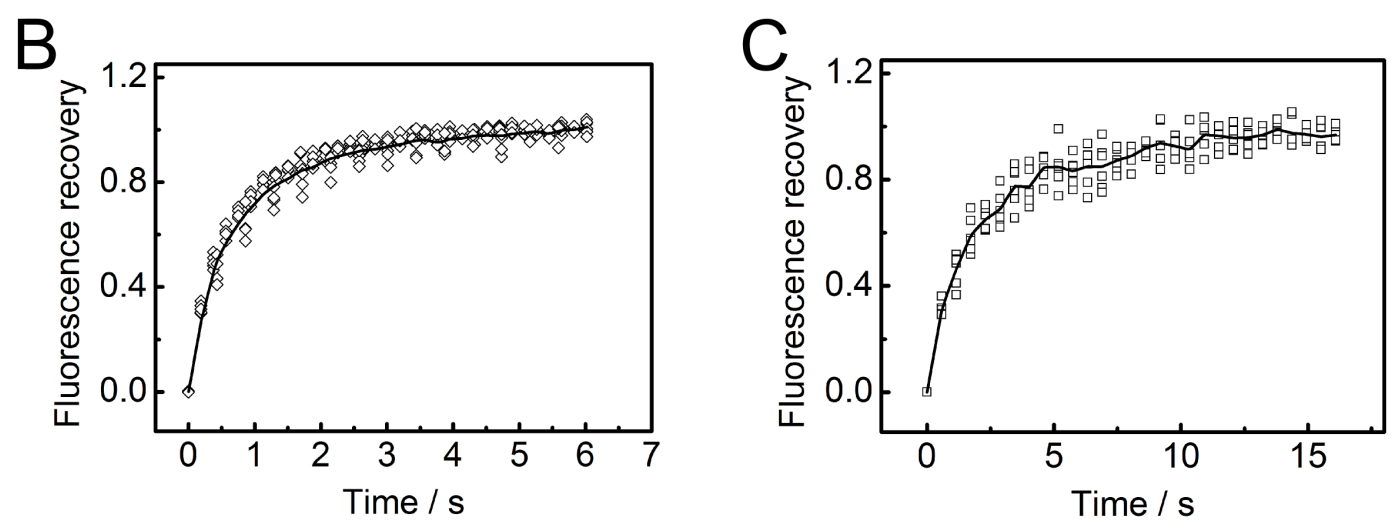

Figure 6.3. (A) Fluorescence micrograph snapshots obtained from an indirect FRAP experiment performed on PSMs composed of DOPC/POPS/POPE/cholesterol (50:19:9:20) doped with $1 \mathrm{~mol} \%$ Atto488-DPPE on a porous substrate with pore diameters of $5 \mu \mathrm{m}$ showing the distribution of fluorophores in a ROI (white circle) with a radius of $2.2 \mu \mathrm{m}$. Scale bar: $5 \mu \mathrm{m}$. Time dependent fluorescence intensity traces obtained from indirect FRAP experiments performed on PSMs composed of DOPC/POPE/POPS/cholesterol (5:2:1:2) doped with either 1 mol \% Atto488-DPPE $(N=9)($ A) or 1 mol\% OG-syx 1A$\operatorname{TMD}(N=6)(\mathrm{B})$. The black curves show the averaged data.

placing circles with a diameter of $5 \mu \mathrm{m}$ that resemble the f-PSM. The area around the circles represent the s-PSM (Fig. 6.4). Independent diffusion coefficients for the f-PSM areas $\left(D_{\mathrm{f}}\right)$ and the s-PSM $\left(D_{\mathrm{s}}\right)$ areas were introduced in the simulation to closely mimic the FRAP experiment with a homogeneous surface concentration in the entire simulation area. An artificial bleach spot was placed in the centered pore with radius $2.2 \mu \mathrm{m}$ (red circle) assuming a Gaussian intensity profile with the concentration $c_{r, 0}$ :

$$
c_{r, 0}=c_{\mathrm{eq}} \cdot \exp \left(-K \cdot \exp \left(\frac{-r^{2}}{w^{2}}\right)\right)
$$

with $c_{\mathrm{eq}}=1, K=2$ and $w=2.2 \mu \mathrm{m}$ as described by Höök et al. [106]. This expression resembles the initial concentration profile within a bleached ROI in the 
FRAP experiments. The fluorescence recovery was subsequently analyzed in the bleach spot as a function of time. Figure 6.5A depicts a series of snapshots taken from a typical FRAP simulation showing the recovery of diffusing particles into the f-PSM.

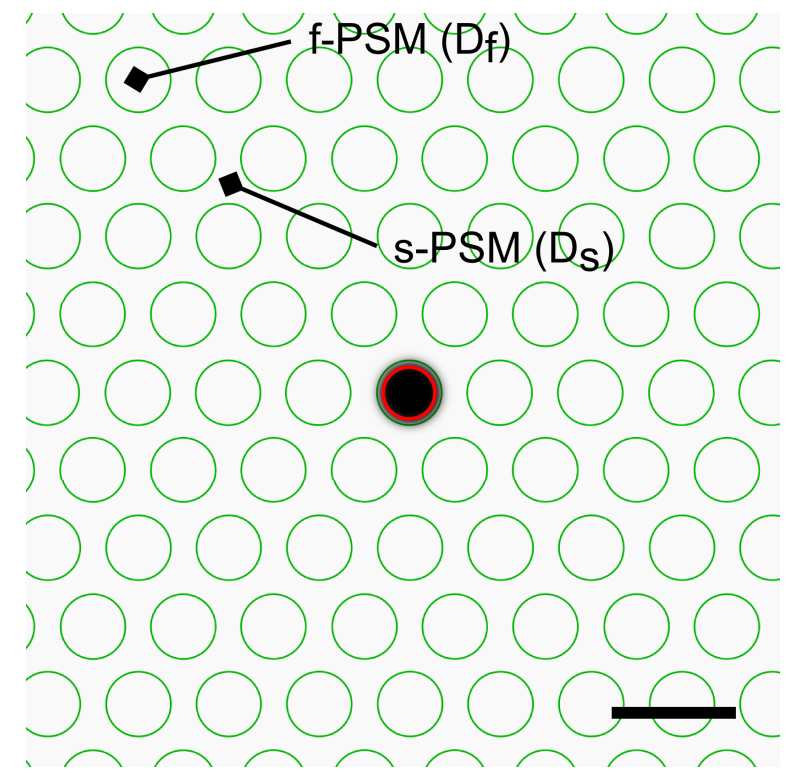

Figure 6.4. Simulated indirect FRAP experiment. The green circles present the f-PSM with a diameter of $5 \mu \mathrm{m}$ while the surrounding area presents the s-PSM. The relative area occupied by the circles yields $36 \%$ as obtained on the PSMs. An artificial bleach spot with a Gaussian profile and radius $2.2 \mu \mathrm{m}$ was placed in the center pore. The fluorescence recovery over time was analyzed in a ROI placed on top of the bleach spot (red circle). Scale bar: $10 \mu \mathrm{m}$.

The recovery of Atto488-DPPE into the f-PSM was simulated with $D_{\mathrm{f}}=7.7 \mu \mathrm{m}^{2} \mathrm{~s}^{-1}$ while the recovery of OG-syx 1A-TMD was simulated with $D_{\mathrm{f}}=3.4 \mu \mathrm{m}^{2} \mathrm{~s}^{-1}$ as these values were determined by FCS for the two compounds in the f-PSM [56]. The diffusion coefficients on the s-PSM $\left(D_{\mathrm{s}}\right)$ were systematically increased from $0.5-4.0 \mu \mathrm{m}^{2} \mathrm{~s}^{-1}$ in increments of $0.5 \mu^{2} \mathrm{~s}^{-1}$ in each simulations to cover a reasonable amount of different diffusion coefficients. Figure $6.5 \mathrm{~B}$ and $\mathrm{C}$ show the resulting simulated recovery curves and the experimental FRAP data. One finds that the Atto488-DPPE recovery data could be modeled most precisely with $D_{\mathrm{s}}=3 \mu \mathrm{m}^{2} \mathrm{~s}^{-1}$ while the OG-syx 1A-TMD recovery curves could be most accurately modeled with $D_{\mathrm{s}}=1 \mu \mathrm{m}^{2} \mathrm{~s}^{-1}$. The obtained diffusion coefficients are in good agreement with those found in glass- and PEGsupported membranes [5, 48, 107]. Also the three times higher mobility of the lipid Atto488-DPPE compared to the transmembrane peptide OG-syx 1A-TMD is consistent with the results obtained by FCS on the f-PSM [56]. 

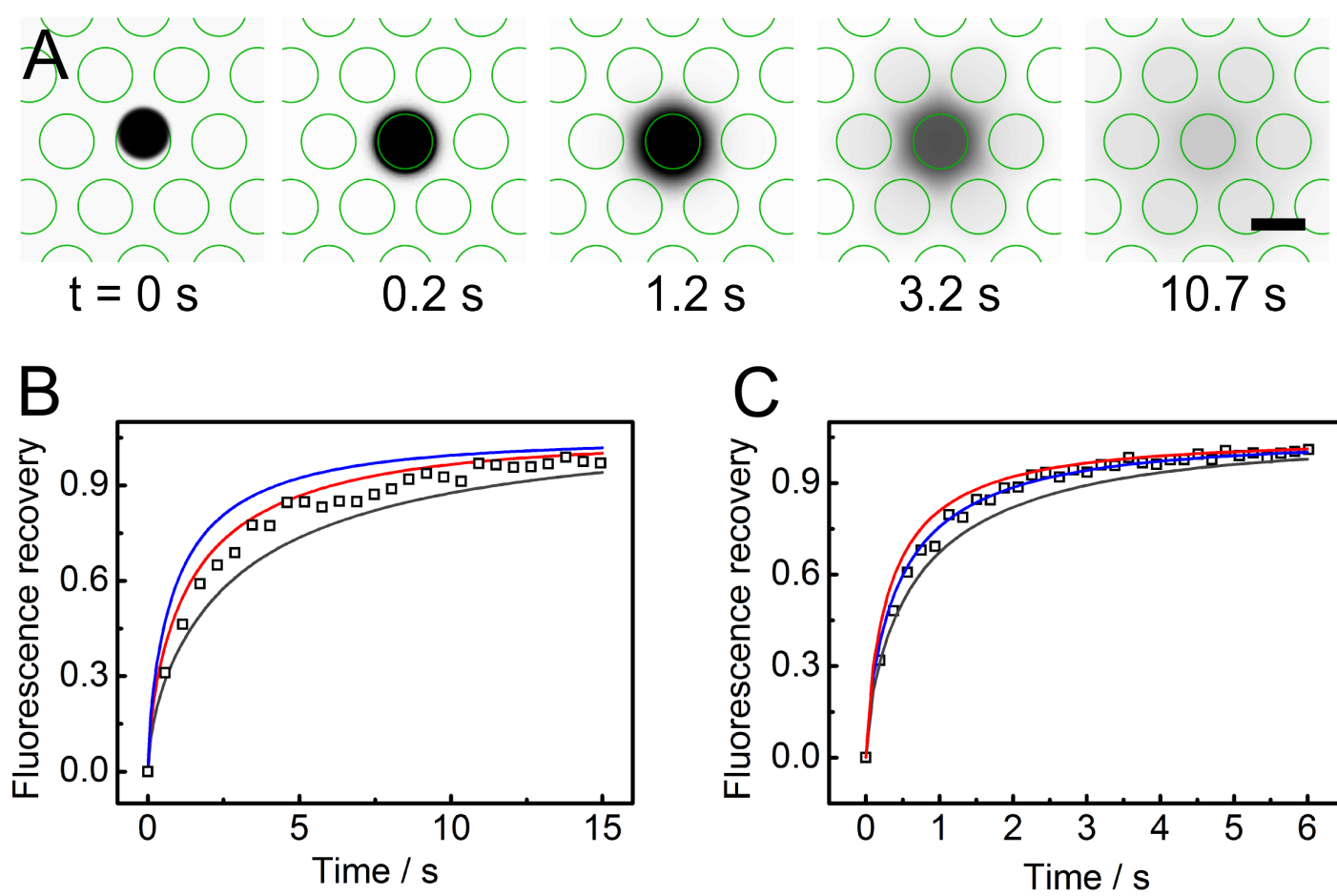

$1.2 \mathrm{~s}$

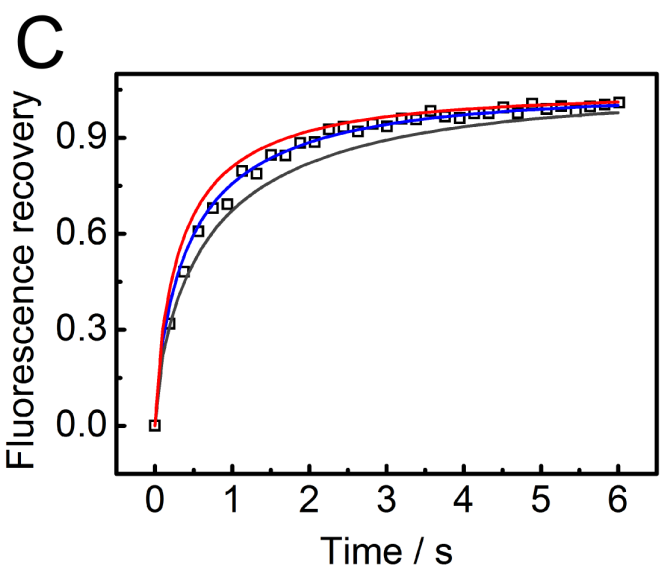

Figure 6.5. (A) Snapshots taken from a simulated FRAP experiment on a porous substrate with pore diameters of $5 \mu \mathrm{m}$ showing the recovery of an artificially bleached spot with radius $2.2 \mu \mathrm{m}$. Scale bar: $5 \mu \mathrm{m}$. (B) Averaged experimental FRAP data of Atto488-DPPE (boxes) and simulated recovery curves with $D_{\mathrm{f}}=7.7 \mu \mathrm{m}^{2} \mathrm{~s}^{-1}$ and $D_{\mathrm{s}}=$ $2 \mu \mathrm{m}^{2} \mathrm{~s}^{-1}$ (gray curve), $D_{\mathrm{s}}=3 \mu \mathrm{m}^{2} \mathrm{~s}^{-1}$ (red curve) and $D_{\mathrm{s}}=4 \mu \mathrm{m}^{2} \mathrm{~s}^{-1}$ (blue curve). The curve with $D_{\mathrm{s}}=3 \mu \mathrm{m}^{2} \mathrm{~s}^{-1}$ models the recovery most precisely. (C) Averaged experimental FRAP data of OG-syx 1A-TMD (diamonds) and simulated recovery curves with $D_{\mathrm{f}}=3.4 \mu \mathrm{m}^{2} \mathrm{~s}^{-1}$ and $D_{\mathrm{s}}=0.5 \mu \mathrm{m}^{2} \mathrm{~s}^{-1}$ (gray curve), $D_{\mathrm{s}}=1 \mu \mathrm{m}^{2} \mathrm{~s}^{-1}$ (blue curve) and $D_{\mathrm{s}}=1.5 \mu \mathrm{m}^{2} \mathrm{~s}^{-1}$ (red curve). The curve with $D_{\mathrm{s}}=1 \mu \mathrm{m}^{2} \mathrm{~s}^{-1}$ models the recovery most precisely. 


\subsection{SNARE-mediated single vesicle fusion assay}

A minimal SNARE fusion machinery, comprised of synaptobrevin 2 (syb 2) and $(\Delta \mathrm{N}$-complex $)$ was functionally reconstituted into small and large vesicles and planar pore-spanning membranes (PSMs) composed of DOPC/POPE/POPS/cholesterol (5:2:1:2) spanning pores with a diameter of $5 \mu \mathrm{m}$. Docking and fusion of differently sized syb 2 vesicles with the s-PSM and the f-PSM was investigated on a single vesicle level with high speed spinning disc confocal microscopy to study the impact of vesicle size and fusion site on the fusion kinetics.

\subsubsection{Analysis of syb 2 vesicle size}

Syb 2 vesicles prepared by extrusion through polycarbonate membranes with nominal pore diameters of $50 \mathrm{~nm}$ and $400 \mathrm{~nm}$ were analyzed by dynamic light scattering (DLS) to determine their mean diameter. Figure 6.6 depicts the intensity-weighted mean diameter distribution of the two syb 2 vesicles. One finds that vesicles extruded through $50 \mathrm{~nm}$ membranes (red curve) exhibit a rather narrow distribution between $50-150 \mathrm{~nm}$ with a peak at $90 \mathrm{~nm}$. The small shoulder around $250 \mathrm{~nm}$ indicates the presence of larger particles, like vesicle aggregates. Vesicles extruded through $400 \mathrm{~nm}$ membranes (blue curve) have a broad intensity distribution between 150 and $400 \mathrm{~nm}$ with a maximum at $240 \mathrm{~nm}$. The DLS measurements confirmed the successful preparation of small syb 2 vesicles with a mean diameter of $90 \mathrm{~nm}$ and large syb 2 vesicles with a mean diameter of $240 \mathrm{~nm}$. 


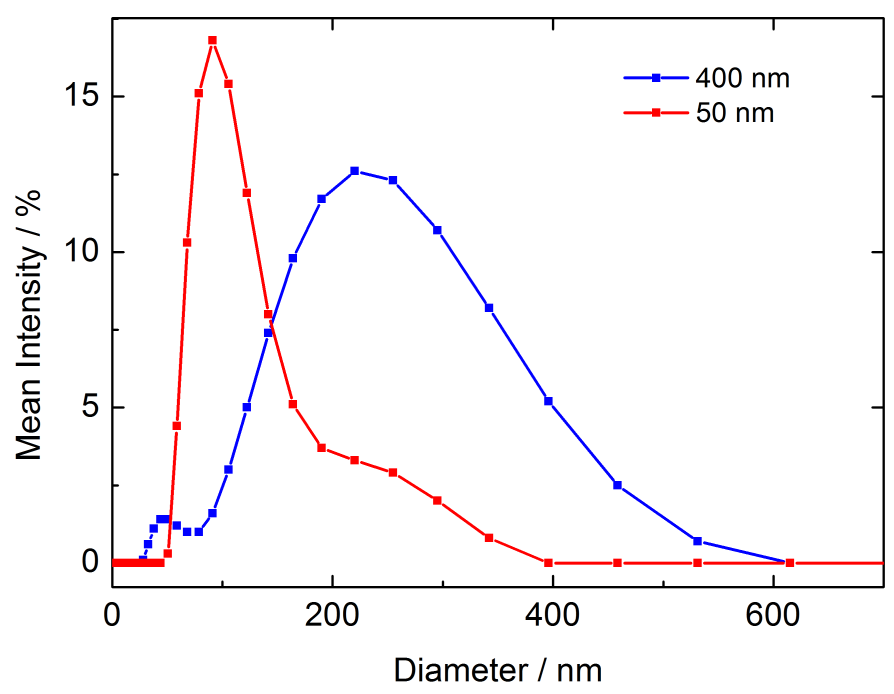

Figure 6.6. Size distribution of syb 2 vesicles extruded through polycarbonate membranes with nominal pore diameters of $50 \mathrm{~nm}$ (red curve) and $400 \mathrm{~nm}$ (blue curve) measured by means of dynamic light scattering.

\subsubsection{Vesicle docking to pore-spanning membranes}

Syb 2 vesicles were fluorescently labeled with $1 \mathrm{~mol} \%$ TR and the PSM with $1 \mathrm{~mol} \%$ Atto488. Prior to the addition of v-SNARE vesicles a snapshot of the PSM patch was recorded to readily identify intact membrane patches and to assign the docking and fusion site of the vesicle from the time series on the PSM. Image acquisition was then started for 155 seconds by recording the TR fluorescence and syb 2 vesicles $(0.5-1 \mu \mathrm{l})$ were added over the individual PSM. The vesicles freely diffuse in the volume above the PSMs until they randomly dock to the planar membrane. Due to the single injection of vesicles at the beginning of each time series, the vesicle concentration above each PSM successively decreases over time since the vesicles distribute within the measuring chamber. To analyze the impact of this gradual vesicle dilution on the docking rate, the cumulative number of docked vesicles was determined as a function of the acquisition time (Fig. 6.7). One finds, that the relative number of docked vesicles drastically decreases over time. After one third of the time series $(50 \mathrm{~s}), 70 \%$ of the analyzed vesicles have already docked to the target-membrane. These findings clearly show that the docking rate is mainly dominated by the dilution of syb 2 vesicles over the PSMs due to the single vesicle injection.

The specificity of SNARE induced docking on the PSMs was analyzed according 


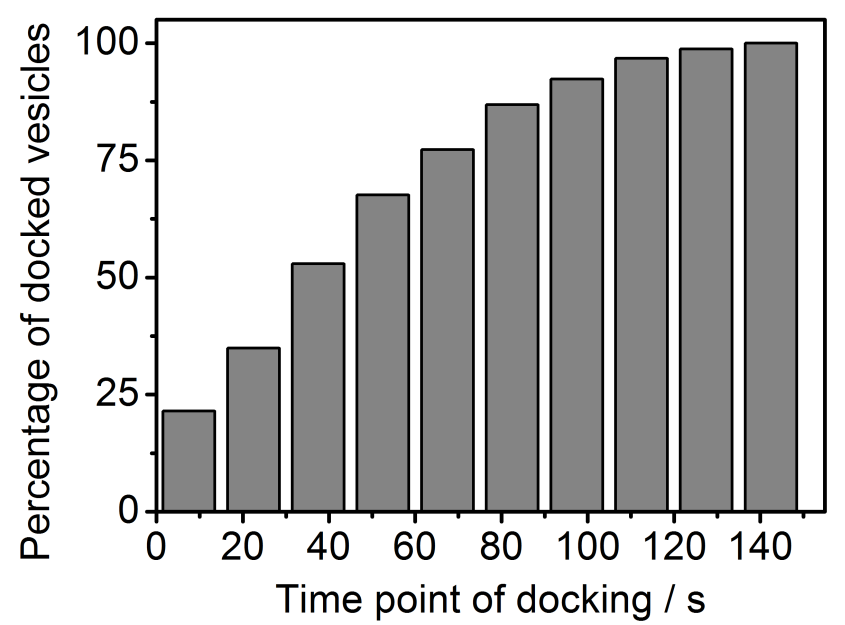

Figure 6.7. Cumulative distribution function of syb 2 vesicles $(N=312)$ with a mean diameter of $240 \mathrm{~nm}$ showing the time point of docking within the 155 seconds long time series. Due to the manual injection of the vesicles at the beginning of each time series, most of the vesicles $(70 \%)$ dock within the first 50 s of the time window.

to the control experiments in the bulk activity assay (Chapter 6.2). $\Delta \mathrm{N}$-complex containing vesicles were pre-incubated with the water soluble syb 2 fragment (residues 1-96) prior to the addition to syb 2 containing PSMs. The syb 2 fragment irreversibly blocks the $\Delta \mathrm{N}$-complex, which is not fusogenic anymore [28]. Docking of blocked $\Delta \mathrm{N}$-complex vesicles to PSMs containing syb 2 could not be observed. These findings are in agreement with those found by the former co-worker Lando Schwenen and prove SNARE-specific docking on the PSMs.

A snapshot taken several seconds after the addition of syb 2 vesicles to a $\Delta \mathrm{N}$-complex containing PSM from a typical fusion experiment is shown in Figure 6.8. The image depicts an overlay of the PSM fluorescence (green), which was taken prior to image acquisition, and the vesicles TR florescence (red) recorded in the time series. One finds that the Atto488 fluorescence of the PSM can only be observed on the free-standing membrane while the fluorescence of the membrane on the support is efficiently quenched by the underlying gold layer and appears dark, a well known phenomenon also reported in literature [5, 104, 105]. The docked syb 2 vesicles however remain visible as they are far enough away from the gold surface and remain visible. Docking of large syb 2 vesicles with a mean diameter of $240 \mathrm{~nm}$, hereafter called $240 \mathrm{~nm}$ vesicles, can be observed on the s-PSM and on the f-PSM (Fig.6.8B) while docking of small syb 2 vesicles with a mean diameter of $90 \mathrm{~nm}$, hereafter called $90 \mathrm{~nm}$ vesicles, could not be 
sufficiently analyzed on the f-PSM due to their substantially lower TR intensity. The TR intensity of a $90 \mathrm{~nm}$ vesicle is 7 times lower than the intensity of a $240 \mathrm{~nm}$ vesicle. In contrast, docking to the s-PSM was observable for both vesicle populations as the gold surface also enhances the fluorescence intensity at distances larger than $20 \mathrm{~nm}$ [105.

The detection of docked vesicles for the later analysis was performed manually. Docked $240 \mathrm{~nm}$ vesicles were further categorized in those fusing with the s-PSM and those fusing with the f-PSM. Analysis of the docking sites of 664 docked vesicles, observed in 7 different preparations on 17 PSM patches, revealed that $16 \%$ of the vesicles $(N=$ 106) initially docked to the f-PSM while the rest docked to the s-PSM. The fraction of vesicles that dock to the f-PSM is by a factor of two smaller than expect by the area percentage occupied by the f-PSMs (36\%). A detachment of docked vesicles could only very rarely be observed during the time series.
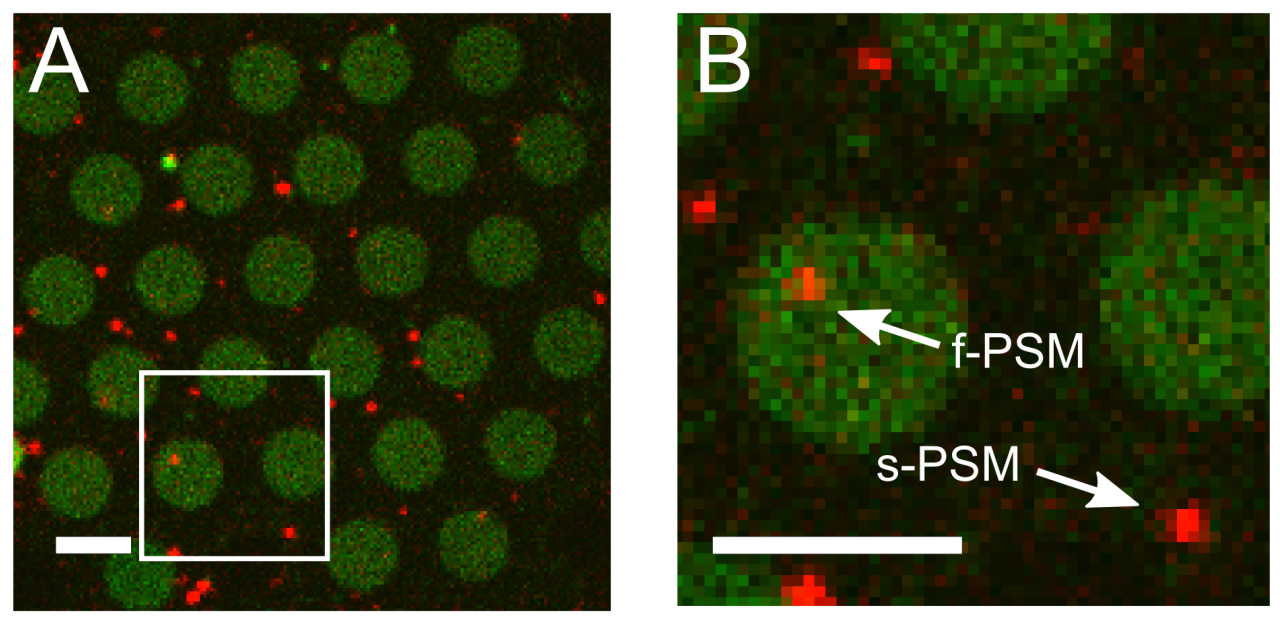

Figure 6.8. (A) Fluorescence micrograph of an Atto488 labeled PSM composed of DOPC/DOPE/DOPS/Chol (5:2:1:2) containing the t-SNARE $\Delta \mathrm{N}$-complex to which TR labeled LUVs composed of the same lipid composition containing synaptobrevin 2 have been docked. (B) Magnification of the marked area (white box) of the fluorescence micrograph shown in (A) highlighting that vesicles dock to supported PSM (s-PSM) and free-standing PSM (f-PSM). Scale bars: $5 \mu \mathrm{m}$.

\subsubsection{Mobility of docked vesicles}

Vesicles that docked to the s-PSM appeared to be fully immobile while those that docked to the f-PSM showed a significant mobility. This discrepancy is analyzed in detail by determining the mobility of s-PSM and f-PSM docked vesicles by single 
particle tracking (SPT) with the software TrackNTrace [68]. TrackNTrace fits the point spread function to the intensity profiles of the vesicles to determine the center of mass with sub pixel resolution. From the individual positions, trajectories were derived visualizing the random walk of the vesicles. Figure 6.9 depicts typical trajectories of a vesicle docked to the s-PSM (A) and a vesicle docked to the f-PSM (B) that are plotted in the corresponding fluorescence micrographs over a time period of several seconds. The trajectory of the s-PSM-docked vesicle appears as a white dot at the center of the vesicle as it is located only within an area, which is much smaller than a single pixel. The small subpixel displacements can be attributed to thermal fluctuations and microscope oscillations. All analyzed s-PSM docked vesicles exhibit identical trajectories, clearly showing that the vesicles do not exhibit any significant lateral movement. This observation is consistent with findings of other groups using solid supported or polymer-cushioned target membranes [48, 50, 53, 108]. In contrast to the s-PSM docked vesicles, those that docked to the f-PSM were found to be highly mobile. Tracking of these vesicles yielded trajectories that covered large areas of the f-PSM as shown in Figure 6.9 B for an exemplary trajectory. The trajectory is only located inside the f-PSM indicating that the vesicle does not leave the f-PSM. This was observed for all f-PSM-docked vesicles. However, most of the vesicles (89\%) were irreversibly immobilized on the pore rim between f-PSM and s-PSM after a certain amount of time.
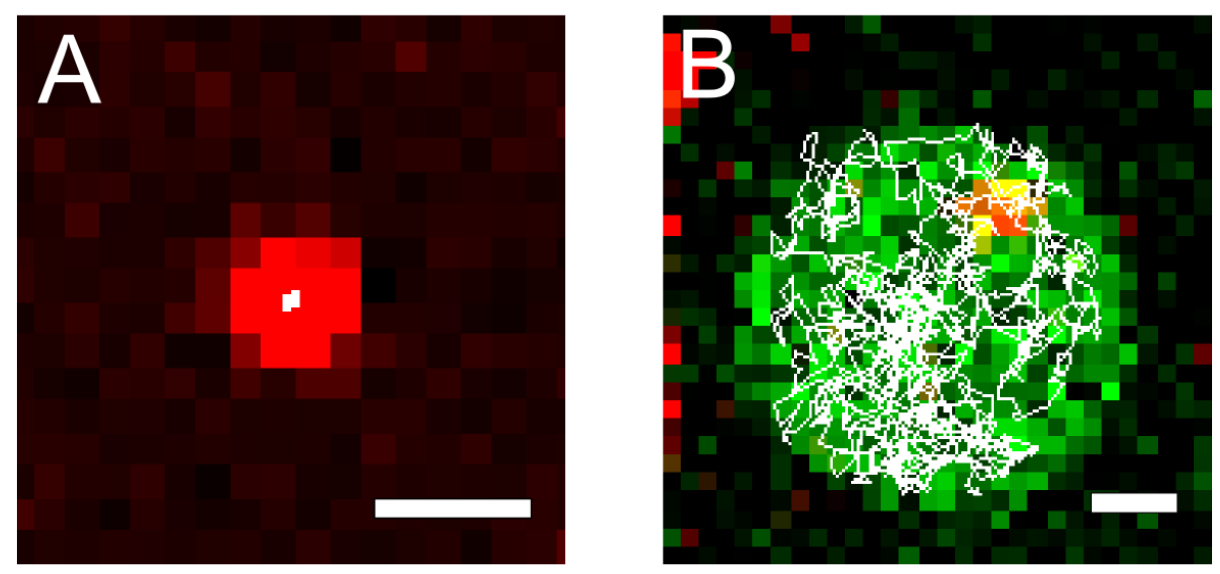

Figure 6.9. Fluorescence micrograph of a $240 \mathrm{~nm}$ syb 2 vesicle docked to a $\Delta \mathrm{N}$-complex containing s-PSM (A) and f-PSM (B) with the corresponding trajectories (white) derived from a time of several seconds. Scale bars: $1 \mu \mathrm{m}$.

As most of the f-PSM docked vesicles indeed diffuse on large parts of the f-PSM but 
yet are immobilized over time on the pore rim, it was investigated, if the vesicles diffuse on all parts of the f-PSM with the same probability. The position of the docked vesicles was analyzed to determine the radial distribution in the f-PSM. The f-PSMs were segmented from the center to the rim in 25 annuli each with a width of $100 \mathrm{~nm}$. The distance of each tracking point of the trajectories to the pore center was then calculated and assigned to the corresponding annulus resulting in the distribution shown in Figure 6.10A. This data is biased as each annulus has a different area. The area of the outer annuli are larger than those of the inner ones. Therefore, the counts per annulus were corrected for the individual sizes yielding the probability density plotted over the normalized pore radius $r / r_{\text {pore }}$ (Fig. 6.10B). The probability to find a vesicle on the edge of the f-PSM is much smaller than to find it near to the pore center. This finding suggests the existence of an increasing energy potential towards the pore rim inside the f-PSM. The energy barrier though does not influence the velocity of the vesicles when diffusing close to the pore rim as the vesicles exhibit a constant velocity in all parts of the f-PSM (Fig. 6.10B).

From the trajectories of the tracked vesicles, mean square displacement (MSD) versus time interval plots were derived to quantitatively analyze the mobility of the vesicles. A typical MSD plot over a large time interval of 23 seconds is depicted in Figure 6.11A. The MSD approaches a constant value on larger time scales (several seconds) indicating confined diffusion of the vesicles as a result of the finite f-PSM area. On shorter time intervals (several hundred milliseconds) the diffusion of the vesicles is unrestricted as shown by three exemplary MSD plots in Figure 6.11C, which are all linear. This is quite reasonable as the f-PSM is significantly larger than the average MSD of the vesicle per time interval $(10 \mathrm{~ms})$. Diffusion coefficients were derived from the slope of MSD plots (4D) from 38 f-PSM docked vesicles, which could be easily tracked with the software TrackNTrace over a maximum time interval of $200 \mathrm{~ms}$ and plotted in the histogram in Figure 6.11C. The data was fitted with a normal distribution resulting in a maximum of $E=0.42 \mu \mathrm{m}^{2} \mathrm{~s}^{-1}\left(\mathrm{SD}=0.15 \mu \mathrm{m}^{2} \mathrm{~s}^{-1}\right)$. As the analyzed vesicles exhibit a rather broad size distribution (140 - $340 \mathrm{~nm}$, see Chapter 6.4.1) it was verified, if the obtained diffusion coefficients are influenced by the size of the tracked vesicles. The TR fluorescence intensity was therefore analyzed in a $6 \times 6$ pixel $^{2}$ ROI, which was placed on the center of each vesicle and corrected for background fluorescence. This is possible as all experiments were conducted with the same microscope adjustments. The total fluorescence of each vesicle, representing its relative size, was plotted versus the corresponding diffusion coefficients (Fig. 6.11D). 

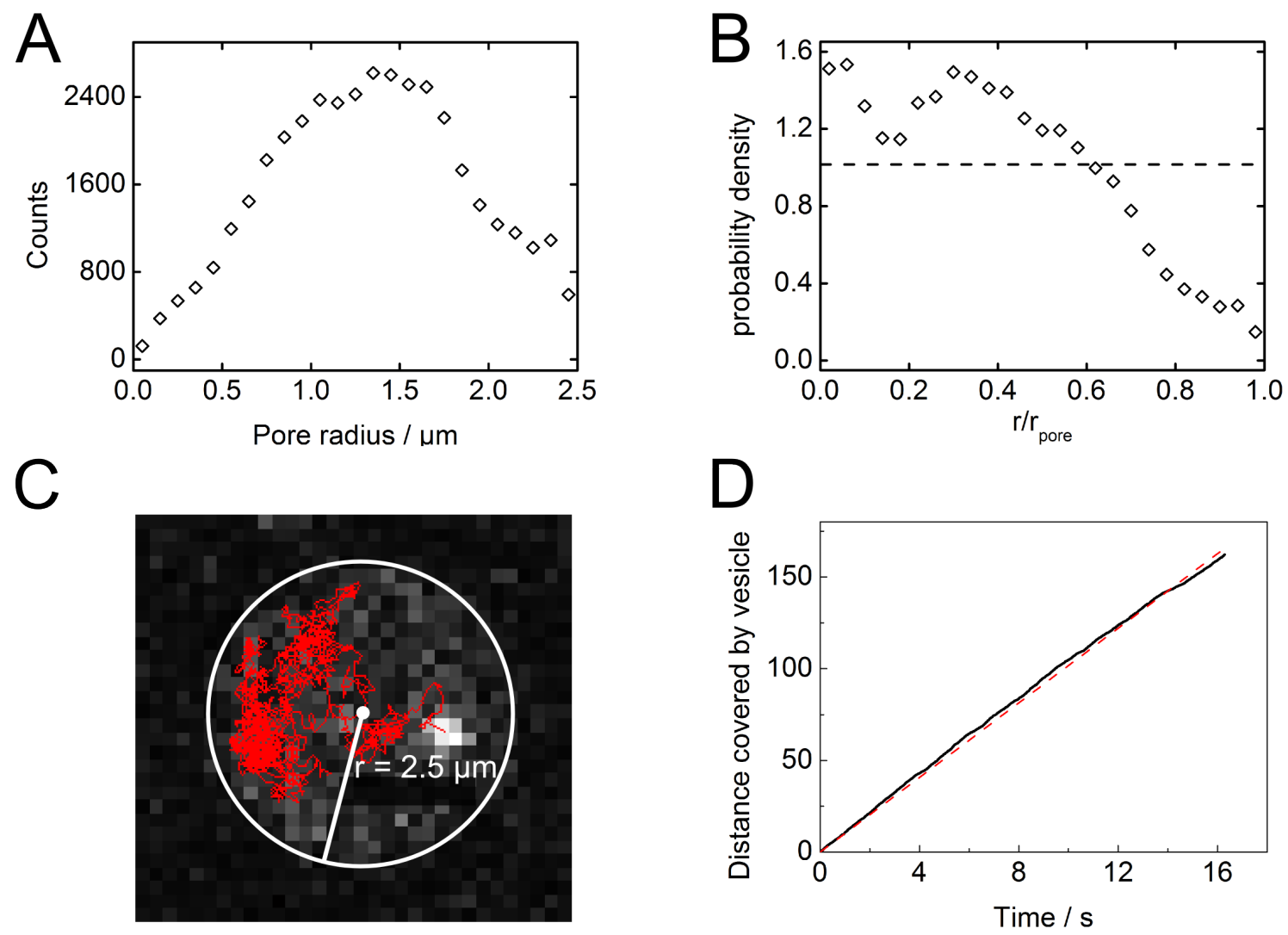

Figure 6.10. (A) Radial distribution of $240 \mathrm{~nm}$ syb 2 vesicles docked to the f-PSM. The data is biased due to the different areas of the annuli. Correction of this data yields the probability density function of the vesicles in the f-PSM (B). (C) Fluorescence micrograph of a $240 \mathrm{~nm}$ syb 2 vesicle docked to a $\Delta \mathrm{N}$-complex containing f-PSM. The trajectory of the diffusing vesicle is shown in red over a time of several seconds. The white circle illustrates the boarder of the f-PSM with a diameter of $5 \mu \mathrm{m}$. (D) Time vs. covered distance of the docked vesicle shown in (C) indicating a constant velocity of the vesicles on all parts of the f-PSM. The hatched red line is a guide for the eye.

Obviously, most of the total fluorescence intensities are located between 4000 - 10000 counts reflecting the size distribution of the vesicles. However, a clear trend that large vesicles diffuse slower than smaller ones cannot be observed from the plot indicating size independent diffusion coefficients of the vesicles. 

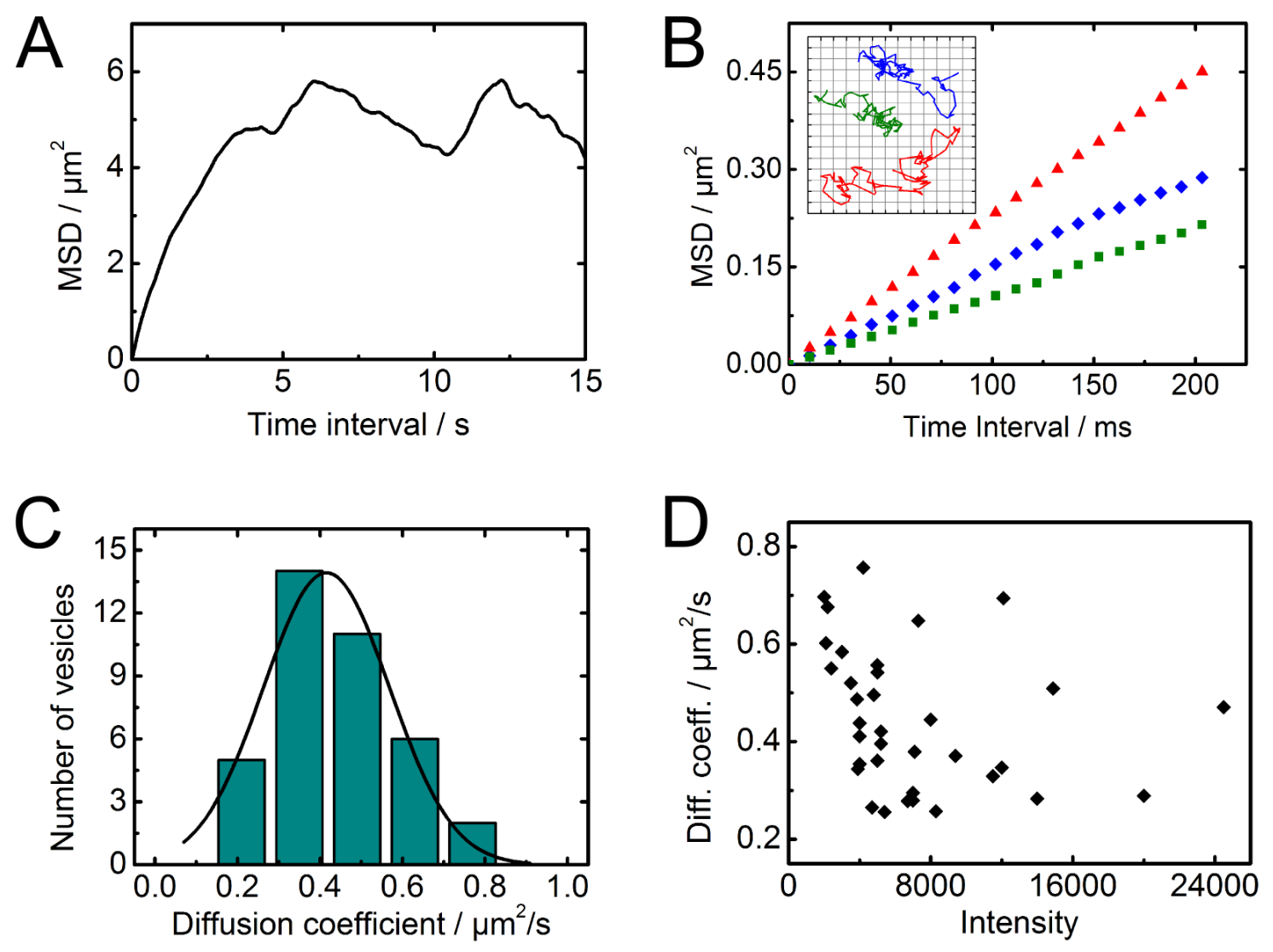

Figure 6.11. Typical MSD versus time plot derived from the trajectory of a f-PSM docked vesicle over a time of 23 seconds. The MSD approaches a constant value $\left(r^{2}=6.25 \mu \mathrm{m}^{2}\right)$, which is typical for confined diffusion. (B) Three exemplaric MSD plots covering a time interval of $200 \mathrm{~ms}$, indicating free diffusion on shorter times scales. From the slope $(4 D)$ of the plots, diffusion coefficients of 0.55 (red), 0.36 (blue) and $0.27 \mu^{2} \mathrm{~s}^{-1}$ (green) could be obtained. The corresponding trajectories are depicted in the inset of $\mathrm{B}$ for a time lapse of $1 \mathrm{~s}$. The grid lines are $200 \mathrm{~nm}$. (C) Histogram analysis of the diffusion coefficients of 38 single vesicles docked to the f-PSM prior to fusion. A Gaussian with a maximum of $0.42 \mu \mathrm{m}^{2} \mathrm{~s}^{-1}\left(\sigma=0.15 \mu \mathrm{m}^{2} \mathrm{~s}^{-1}\right)$ (black line). (D) Total TR intensity representing the relative size of $38 \mathrm{f}-\mathrm{PSM}$ docked vesicles plotted against their corresponding diffusion coefficients. 


\subsubsection{Fusion of vesicles with pore-spanning membranes}

Fusion of $90 \mathrm{~nm}$ and $240 \mathrm{~nm}$ syb 2 vesicles with the PSM was analyzed in a time resolved manner. As mentioned before, on the f-PSM only large $240 \mathrm{~nm}$ vesicles could be analyzed while on the s-PSM also the smaller $90 \mathrm{~nm}$ vesicles were detectable. Fusion events on the f-PSM could only very rarely be observed, as $89 \%$ of the vesicles that initially docked to the f-PSM $(N=106)$ were immobilized on the pore rim between the s-PSM and f-PSM after some time (Chapter 6.3). As most of these immobilized vesicles could not regain their mobility, they were consequently analyzed as s-PSM fusion events. This feature and the $50 \%$ lower docking probability on the f-PSM (Chapter 6.4 .2 explains why in total only $3 \%$ of the docked vesicles $(N=664)$ eventually fused with the f-PSM. In comparison, $47 \%$ of the docked vesicles fused with the s-PSM while the rest remained docked until the end of the time series.

Fusion of vesicles with the PSM was investigated by analyzing the TR fluorescence over time in a $4 \times 4$ pixel $^{2}$ ROI on the center of mass of the vesicle. Figure 6.12 depicts a series of fluorescence micrographs of a vesicle that fuses with the f-PSM and the corresponding TR fluorescence intensity. The time point of fusion was set to $t=0 \mathrm{~s}$. Due to the high mobility, the vesicles had to be tracked during the analysis inside the f-PSM. Docking of the vesicle in the ROI leads to an increase in TR intensity to a constant level $(t=-4.7 \mathrm{~s})$. The onset of fusion $(t=0 \mathrm{~s})$ is indicated by a sudden decrease in TR intensity to the pre-docking intensity when the lipid material radially distributes in the surrounding planar membrane. The time between the initial docking and onset of fusion is called docking time $\left(t_{\text {dock }}\right)$ and is analyzed in detail in Chapter 6.4.7.

The radial TR distribution around the fusing vesicles is a certain indicator for successful lipid mixing between the vesicle membrane and the PSM and can be observed in a rectangular donut shaped ROI placed around the vesicle as shown in Figure 6.13A. The donut-shaped ROI (2) has a size of $8 \times 8 \mathrm{pixel}^{2}$ with a width of 2 pixel while the enclosed center ROI (1) has a size of $4 \mathrm{x} 4 \mathrm{pixel}^{2}$. Time resolved TR fluorescence intensity traces observed in the donut-shaped ROI and in the enclosed center ROI are depicted in Figure 6.13B. Upon onset of fusion $(t=0 \mathrm{~s})$, the TR intensity in the center ROI (black) decays to the pre-docking intensity. At the same time, TR diffuses from the vesicle into the donut-shaped ROI (gray), resulting in an increase of TR intensity, which then immediately decreases again as the TR distributes further in the planar membrane. It was analyzed, whether the TR decay in the center ROI resembles a purely diffusive character by interpreting the vesicle as a lipid disc source that diffuses 


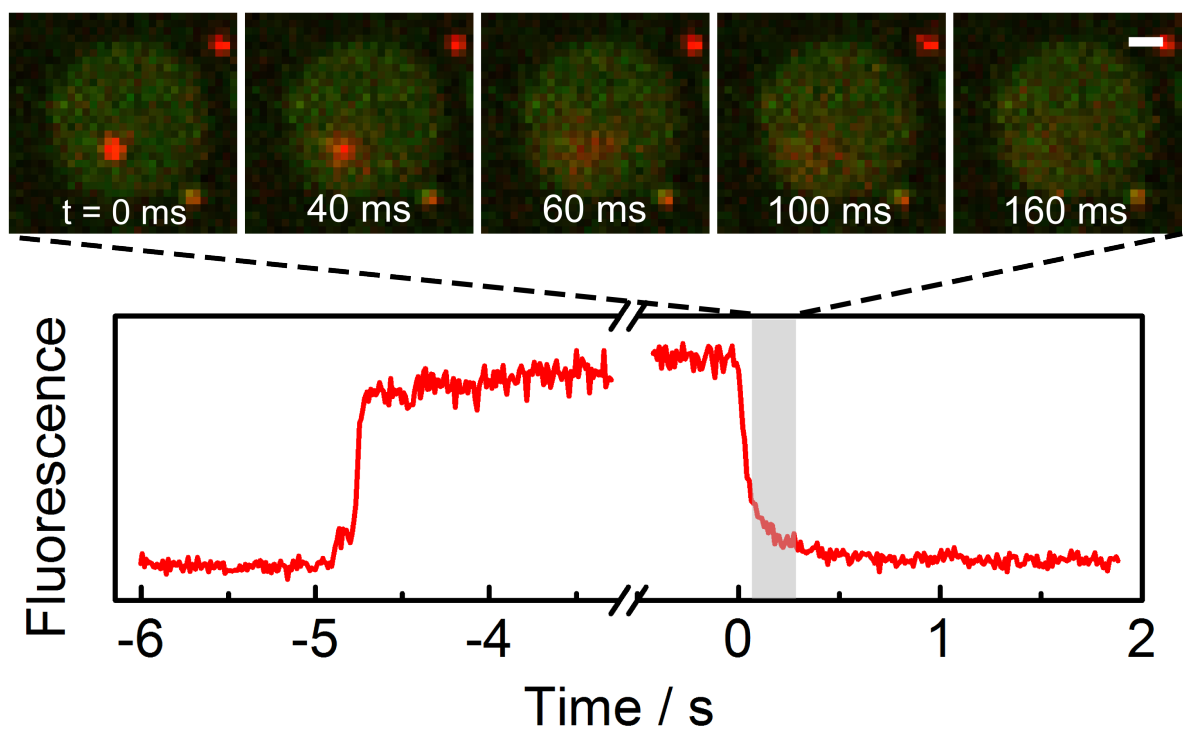

Figure 6.12. Fluorescence micrograph sequence of a $240 \mathrm{~nm}$ syb 2 vesicle composed of DOPC/POPE/POPS/cholesterol (5:2:1:2) labeled with $1 \mathrm{~mol} \%$ TR fusing with a planar $\Delta \mathrm{N}$-complex containing f-PSM and the corresponding TR fluorescence intensity trace recorded in a $2 \times 2$ pixel $^{2}$ ROI on top of the vesicle. The PSM is composed of the same lipid mixture as the vesicle and labeled with $1 \%$ Atto488. Scale bar: $1 \mu \mathrm{m}$.

into the surrounding PSM with a given diffusion coefficient $D$. The diffusion of a radial disc source with radius $r$ diffusing into the surrounding membrane according to Fick's second law can be described by:

$$
I(t)=1-\exp \left(-\frac{r^{2}}{4 D t}\right)
$$

with $I(t)$ the TR intensity as a function of time, $r$ the radius of the disc source and $D$ the diffusion coefficient [109]. The TR decay shown in Figure 6.13B was modeled with Equation 6.3 assuming a ROI radius with $r=0.5 \mu \mathrm{m}$ and a diffusion coefficient of $D=2 \mu \mathrm{m}^{2} \mathrm{~s}^{-1}$ (red curve). The decay can be modeled very well proving a diffusive distribution of TR in the surrounding membrane upon lipid mixing. The assumed diffusion coefficients of $2 \mu \mathrm{m}^{2} \mathrm{~s}^{-1}$ does not exactly resemble the diffusion coefficients of the f-PSM as the exact radius of the fusing vesicle is unknown and the fluorophores diffuse through the fusion stalk rather than the PSM.

Fusion of 90 and $240 \mathrm{~nm}$ vesicles with the s-PSM was analyzed according to vesicles that fused with the f-PSM by reading out the TR intensity over time (Fig. 6.15). Docking of the vesicle is observable by the increase in TR fluorescence while the onset of fusion is indicated by the TR decay to the pre-docking intensity as shown for the 

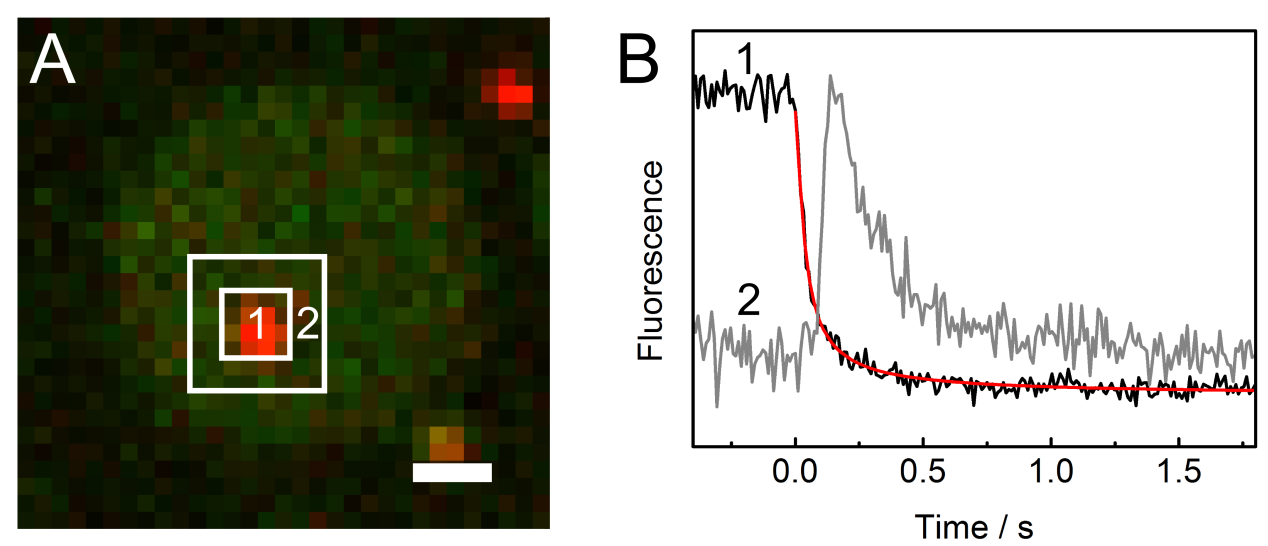

Figure 6.13. Analysis of lipid mixing on the f-PSM. (A) Fluorescence micrograph depicting a $240 \mathrm{~nm}$ syb 2 vesicle docked to the f-PSM prior to fusion. Scale bar: $1 \mu \mathrm{m}$. (B) Time resolved TR fluorescence intensity traces recorded from the center ROI (1, black curve) and from the donut-shaped ROI (2, grey curve) shown in (A). The TR decay recorded in the center ROI was modeled with equation 6.3 assuming a radius of $r$ $=0.5 \mu \mathrm{m}$ and a diffusion coefficient of $D=2 \mu \mathrm{m}^{2} \mathrm{~s}^{-1}$ (red curve).

events on the f-PSM. In contrast to vesicles that fused with the f-PSM, here, the radial TR distribution could not be observed in a donut-shaped ROI placed around the vesicle due to the gold layer that quenches the fluorescence on the planar membrane (Fig. $6.15 \mathrm{~A} / \mathrm{B})$. The gold-induced fluorescence enhancement and quenching additionally affects the kinetics of the TR decay in the center ROI, which is why the TR decay cannot be adequately modeled on the s-PSM with equation 6.3 assuming the same parameters $\left(r=0.5 \mu \mathrm{m}\right.$ and $\left.D=2 \mu \mathrm{m}^{2} \mathrm{~s}^{-1}\right)$ as successfully applied on the f-PSM (Fig. 6.15). 


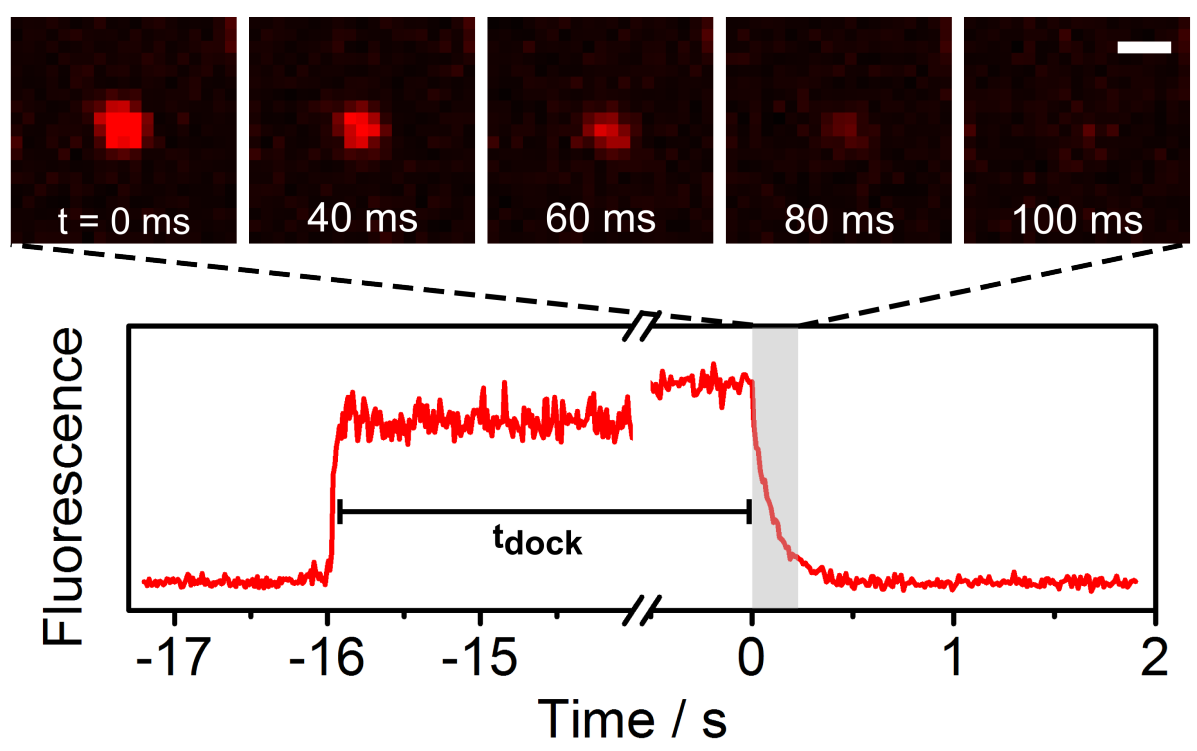

Figure 6.14. Fluorescence micrograph sequence of a $240 \mathrm{~nm}$ syb 2 vesicle composed of DOPC/POPE/POPS/cholesterol (5:2:1:2) labeled with $1 \mathrm{~mol} \%$ TR fusing with a planar $\triangle \mathrm{N}$-complex containing s-PSM and the corresponding TR fluorescence intensity trace recorded in a $2 \times 2$ pixel $^{2}$ ROI on top of the vesicle. The PSM is composed of the same lipid mixture as the vesicle and labeled with 1\% Atto488. Scale bar: $1 \mu \mathrm{m}$.
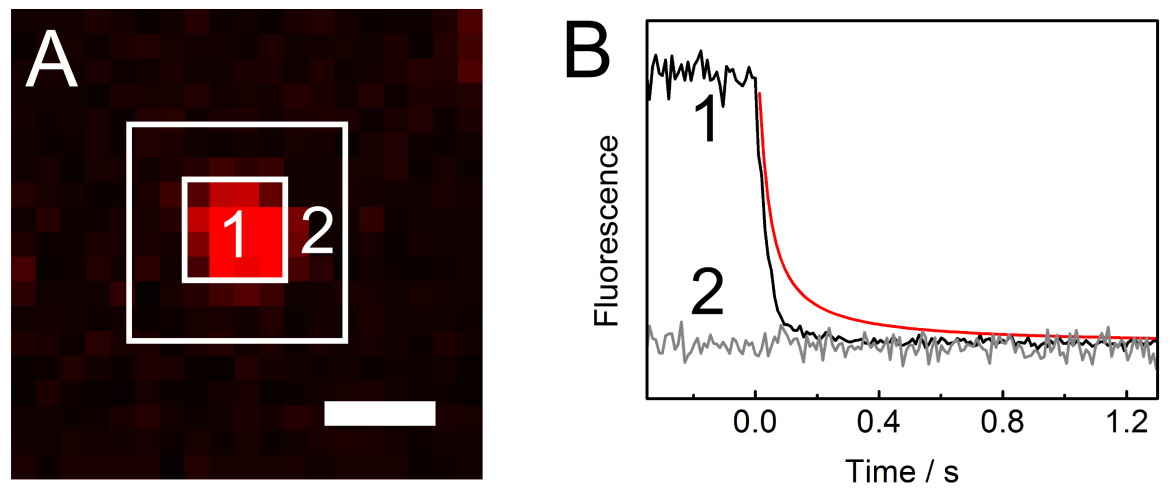

Figure 6.15. Analysis of lipid mixing on the s-PSM. (A) Fluorescence micrograph depicting a syb 2 vesicle docked to the s-PSM prior to fusion. Scale bar: $1 \mu \mathrm{m}$. (B) Time resolved TR fluorescence intensity traces recorded from the center ROI (1, black curve) and from the donut-shaped ROI (2, grey curve) shown in (A). The TR decay recorded from the center ROI was modeled with equation 6.3 assuming a radius of $r=0.5 \mu \mathrm{m}$ and a diffusion coefficient of $D=2 \mu \mathrm{m}^{2} \mathrm{~s}^{-1}$ (red curve). 


\subsubsection{Post fusion structures of vesicles}

Fusion of vesicles with the target-membrane involves an $\Omega$-like post fusion structure, which is formed by the fusion pore [18, 110, 111]. The vesicle content can subsequently be released through the fusion pore leading to a shrinking and merging of the vesicle into the target-membrane. This process was analyzed in more detail harnessing the height-dependent fluorescence de-quenching of Atto488 on the s-PSM. Upon onset of fusion, 2D lipid diffusion through the fusion pore sets in, resulting in the radial distribution of TR as described in Chapter 6.4.4. At the same time, Atto488 diffuses from the planar membrane into the $\Omega$-shaped vesicle structure and is de-quenched due to its greater distance to the gold surface as cartooned in Figure 6.16A. It is important to note that the Atto488 dequenching signal can only be observed on the s-PSM, restricting this analysis to events on the f-PSM.

$240 \mathrm{~nm}$ syb 2 vesicles (p:1 1:500) doped with $1 \mathrm{~mol} \%$ TR were fused with PSMs carrying the t-SNARE $\Delta$ N-complex (p:1 1:500) composed of DOPC/POPE/POPS/cholesterol (5:1:2:2) doped with $1 \mathrm{~mol} \%$ Atto488. Fusion of the vesicles was monitored with two-color spinning disc microscopy with a time resolution of $20 \mathrm{~ms}$, where Atto488 and TR were excited and recorded simultaneously. As the Atto488 and TR fluorescence was each aligned onto one half of the camera detector, the timing between the two channels was exactly the same. Figure 6.16B and C depict two exemplaric Atto488 and TR intensity traces analyzed from a $4 \times 4$ pixel $^{2}$ ROI on the center of each vesicle. The onset of fusion, indicated by the TR decay, was set to $t=0 \mathrm{~s}$. One observes a constant Atto488 background fluorescence during the entire docking time of the vesicles in all events, proving that no lipid material is exchanged between the two membranes. Upon fusion, Atto488 diffuses into the vesicle and is dequenched resulting in an increasing fluorescence signal. The time traces shown in Figure 6.16 B exhibit only a very short Atto488 signal, suggesting a fast vesicle merging, while the traces in Figure $6.16 \mathrm{C}$ show an Atto488 signal with a lifetime of several seconds, slightly deceasing over time, which is interpreted as a slowly merging vesicle. $47 \%$ of the analyzed fusion events $(N=60)$ showed a fast vesicle merging with lifetimes of $<1 \mathrm{~s}$, while the residual $53 \%$ merged significantly slower into the membrane with lifetimes of several seconds. In some events merging was even fully restricted, as the Atto488 fluorescence remained de-quenched until the end of the time series. 

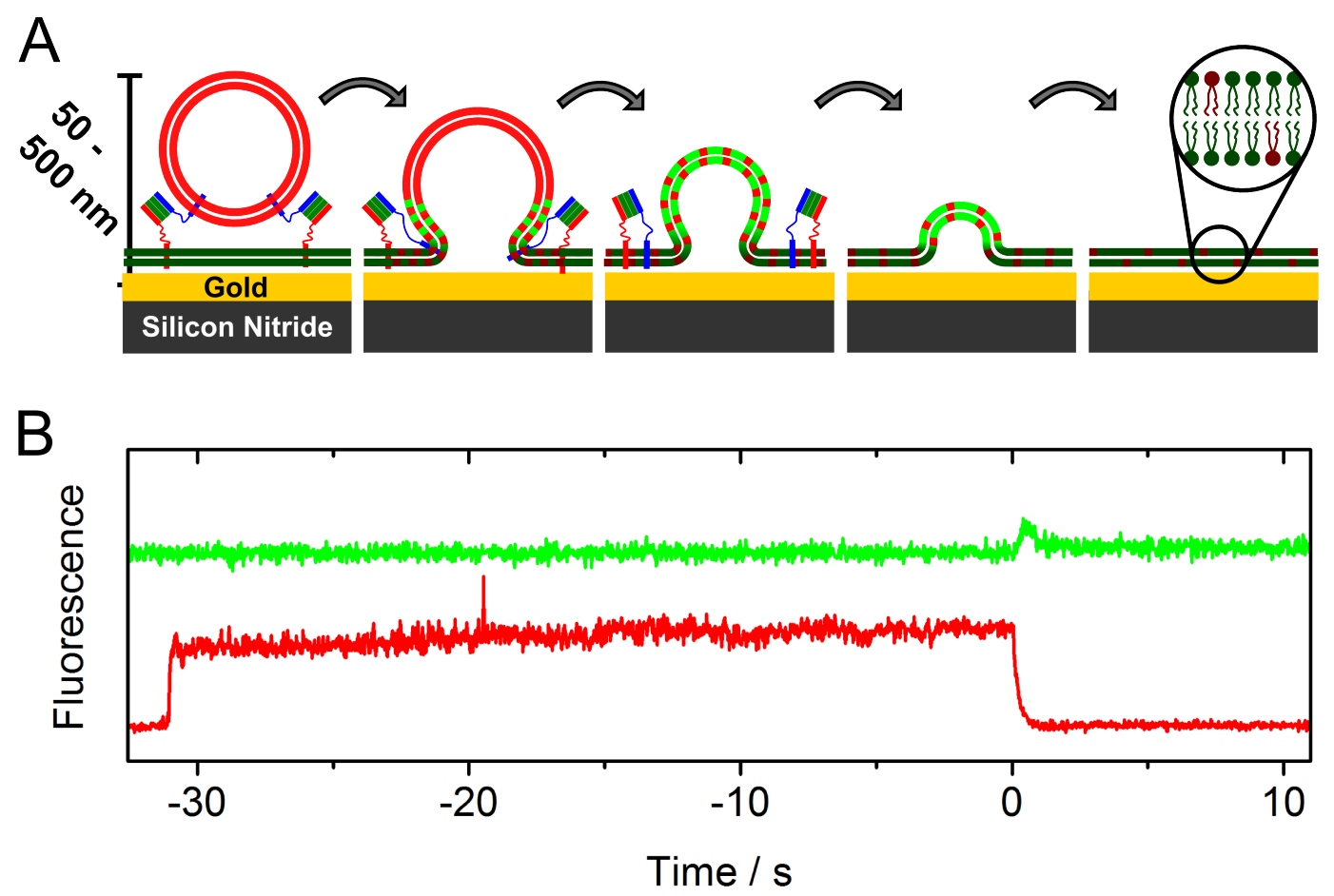

C

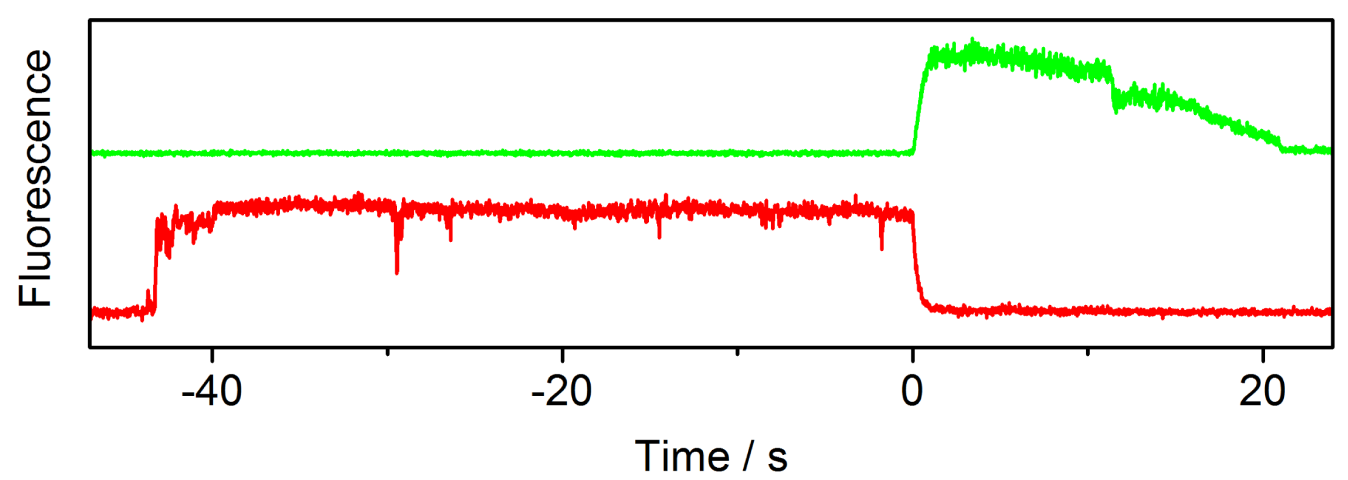

Figure 6.16. Analysis of the vesicular post-fusion structure on the s-PSM. (A) Schematic drawing of a vesicle fusing with the s-PSM. The TR-DHPE labeled vesicle is docked to the Atto488-DPPE labeled PSM. Upon fusion, TR-DHPE diffuses out of the vesicle ghost into the planar PSM and is quenched while Atto488-DPPE simultaneously diffuses into the vesicle ghost and is de-quenched. Time resolved TR-DHPE and Atto488 fluorescence intensity traces of vesicles composed of DOPC/POPE/POPS/cholesterol (5:2:1:2) with a mean diameter of $240 \mathrm{~nm}$ of a vesicle that rapidly collapsed into the target membrane (B) and one that slowly collapsed into the target membrane (C). 


\subsubsection{Intermediate TexasRed intensity levels of vesicles}

Fusion of 312 syb 2 vesicles with a mean diameter of $240 \mathrm{~nm}$ and 340 vesicles with a mean diameter of $90 \mathrm{~nm}$ with PSMs was observed by analyzing the TR fluorescence intensity from the center of each vesicle as shown in Chapter 6.4.4. When the TR intensity recovered to the pre-docking intensity within a single step as shown in Figure 6.17A, indicating a full TR release, it was assumed that no intermediates occur within the $10 \mathrm{~ms}$ time resolution of the measurement. These events are therefore called full fusion events. $87 \%$ of the $240 \mathrm{~nm}$ and $86 \%$ of the $90 \mathrm{~nm}$ vesicles showed full fusion. The other events exhibited intermediate TR intensity levels located between the docking and the pre-docking intensity as shown in Figure 6.17B. It is important to note that such events only occurred on the s-PSM. On the f-PSM only full fusion events were observed. Events with an incomplete TR release are called intermediate fusion events and were further characterized by determining the background corrected relative TR intensity of the intermediate fluorescence level (Fig. 6.17 C and D). The relative TR intensity displays the amount of remaining vesicular lipid material that was not fused with the target-membrane. One finds that nearly all intermediate intensities are distributed below $50 \%$ regardless of the vesicle size. While the $90 \mathrm{~nm}$ vesicles have a relative TR intensity distribution with a maximum at around $25 \%$, the larger $240 \mathrm{~nm}$ vesicles show a distribution with a clear maximum at around $25 \%$ and a small shoulder around $50 \%$. $64 \%$ of the intermediate levels arising from the $240 \mathrm{~nm}$ vesicles and $32 \%$ of the events arising from the $90 \mathrm{~nm}$ vesicles even recovered to the pre-docking intensity in a second step (Fig. 6.17B). The intermediate TR intensity can therefore be interpreted as a semi-stable fusion state. The lifetimes of these states were analyzed and are plotted in Figure 6.17 $\mathrm{E}$ and $\mathrm{F}$ for the two $90 \mathrm{~nm}$ and $240 \mathrm{~nm}$ vesicle populations. The lifetimes are distributed between 2 - 90 seconds and do not show an exponential decay, which would classify the intermediate TR level as fusion intermediates with a single rate constant. 

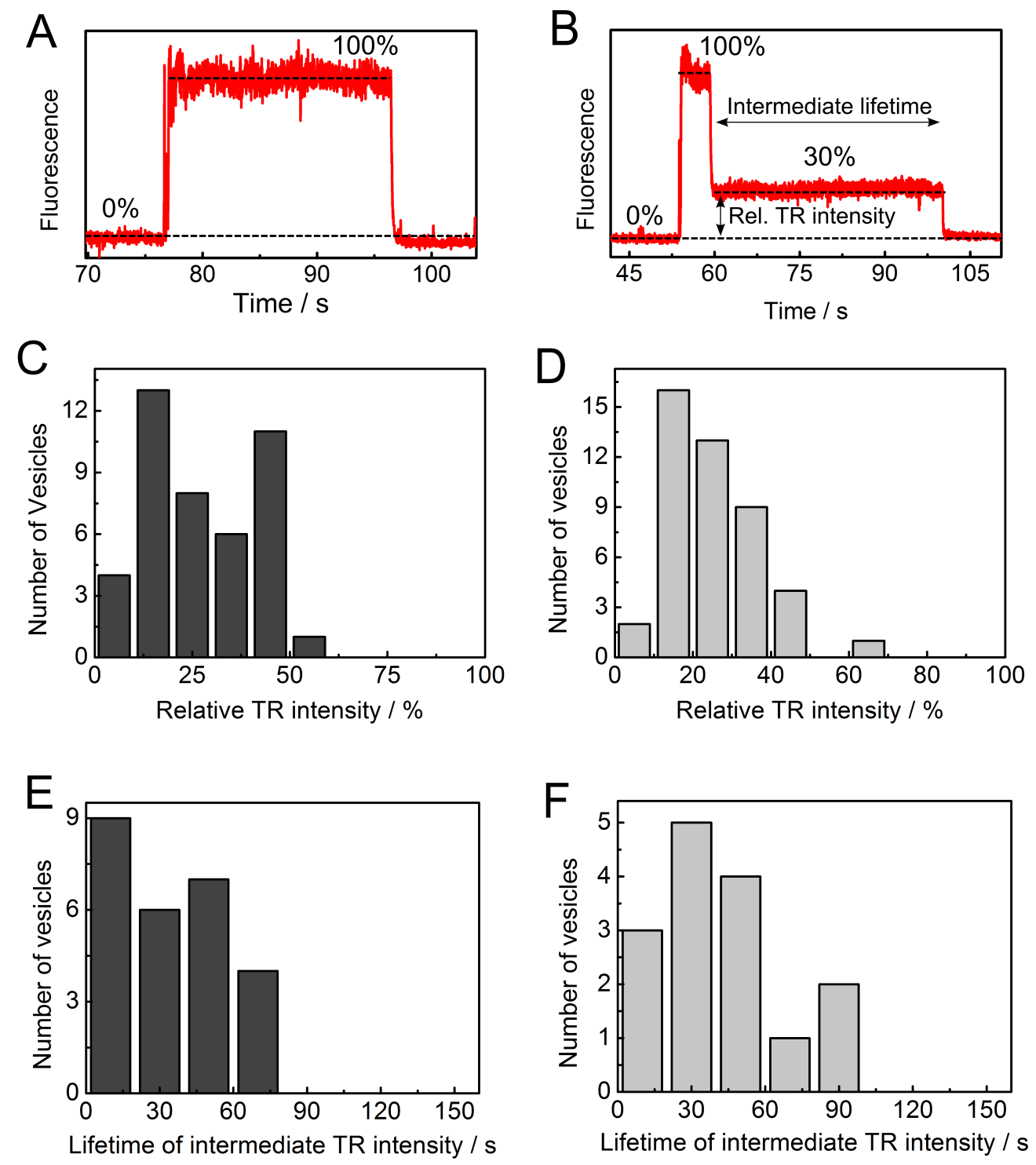

Figure 6.17. Analysis of intermediate fusion events on the s-PSM. TR fluorescence intensity vs. time traces of a vesicle exhibiting no intermediate TR intensity level after the onset of fusion (A) and one that points up an intermediate TR intensity level at around $30 \%$, which recovers to the pre-docking intensity $(0 \%)$ in a second step (B). Histogram analysis of the relative TR intensities of $240 \mathrm{~nm}$ vesicles $(N=56)(\mathrm{C})$ and $90 \mathrm{~nm}$ vesicles $(N=45)(\mathrm{D})$. The bin size is $10 \mathrm{~s}$. Histogram analysis of the lifetimes of the intermediate TR intensity levels of $240 \mathrm{~nm}(N=26)(\mathrm{E})$ and $90 \mathrm{~nm}$ vesicles $(N=$ 16) (F). The bin size is $20 \mathrm{~s}$. 
On this account, it was further investigated, whether the intermediate TR intensity level can be correlated with the initial total fluorescence intensity of the vesicles. The background corrected mean integral fluorescence intensity of the docked $90 \mathrm{~nm}$ and $240 \mathrm{~nm}$ vesicles was analyzed in a $6 \times 6$ pixel $^{2}$ ROI and separately plotted for intermediate fusion and full fusion events (Fig. 6.17A/B). A comparison of the fluorescence intensities from different experiments is possible as all measurements were performed with the same microscope and laser adjustments. A log-normal distribution (red and blue curves) was fitted to the data yielding mean values of 400 counts (240 nm vesicle) and 300 counts for the full fusion events and 300 counts ( $240 \mathrm{~nm}$ vesicle) and 240 counts (90 nm vesicle) for the intermediate fusion events. To verify if the distributions are different, two sample Kolmogorow-Smirnow-Tests (KS-Test) with a $5 \%$ significance level were performed. The KS-Test rejected the null hypothesis, indicating no significant differences in the intensity distributions. It can therefore not be safely inferred that vesicles with intermediate fusion states are brighter than those fully fusing with the target-membrane.
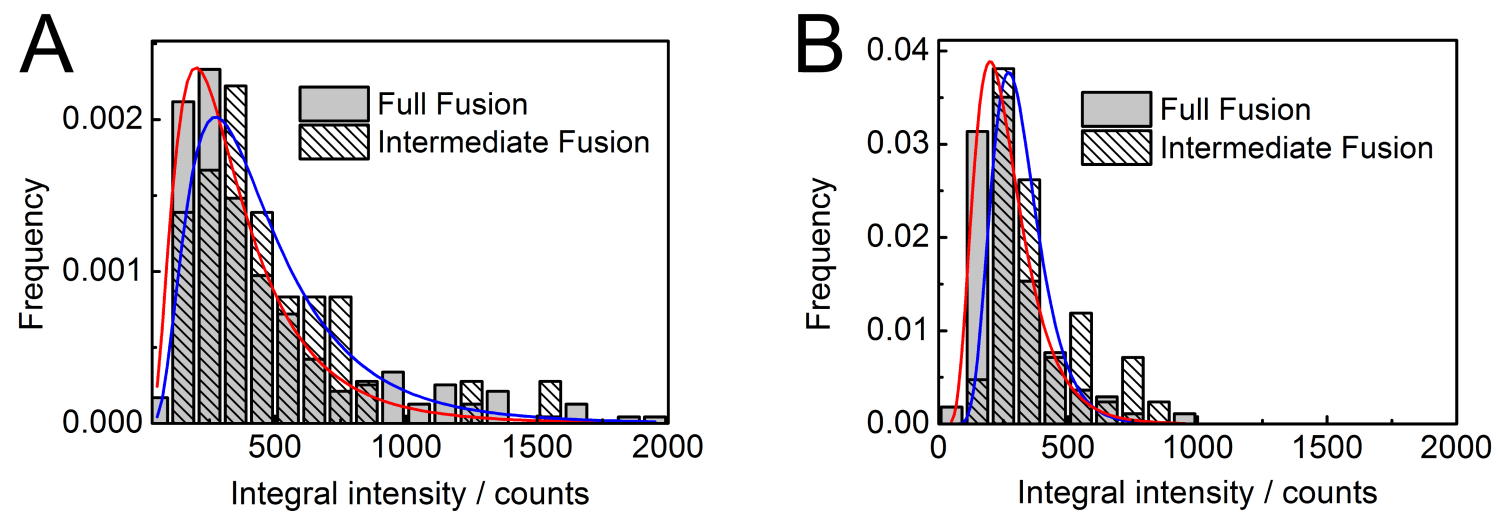

Figure 6.18. Intensity analysis of syb 2 vesicles showing full fusion and intermediate fusion on the s-PSM. (A) Mean integral intensity distribution of 236 full fusion events (gray bars) and 36 events with an intermediate TR intensity level (striped bars) of $240 \mathrm{~nm}$ vesicles. (B) Mean integral intensity distribution of 274 full fusion events (gray bars) and 42 events with an intermediate TR intensity level (striped bars) of $90 \mathrm{~nm}$ vesicles. The intensities were fitted with a log-normal distribution (red and blue curve) to obtain mean integral intensities. A two-sample KS-Test rejects verified no differences between the distributions at a $5 \%$ significance level. 


\subsubsection{Docking times of small and large vesicles}

The docking times of small $90 \mathrm{~nm}$ and large $240 \mathrm{~nm}$ vesicles fusing with the s-PSM and f-PSM were analyzed to shed some light onto the kinetics of the fusion process. The docking time is defined as the time interval between docking and the onset of fusion and was analyzed from the time resolved TR intensity traces recorded on top of each vesicle (Chapter 6.4.4). Figure 6.19 displays the docking time distribution with a bin size of $10 \mathrm{~s}$ of large $2400 \mathrm{~nm}$ vesicles (A) and small $90 \mathrm{~nm}$ vesicles (B) fusing with the s-PSM. The docking times are distributed between 1-150 seconds with a maximum at 12.5 and 16.7 seconds, respectively. As the docking times are distributed over the entire time series, events occurring at larger times are underestimated due to the finite observation window of the measurement (155s). A statistical correction would be necessary to account for this effect. Yet, such a statistical correction would only yield reasonable results, if a constant docking rate can be assumed, which is not the case in the experiments as shown in Chapter 6.4.2. Therefore a correction is not employed for the presented data. In both docking time histograms, a rise and decay can be observed in the frequency of events. Such a distribution is typical for processes involving multiple steps as a mechanism involving only a single step would result in an exponential decay [112]. A lag time in which faster fusion events might be unnoticed, can be excluded due to the high time resolution of the measurement, which is 1000 times larger than the bin size in the histogram. To analyze the number of reaction steps hidden in the docking time distribution, the docking times were modeled with a gamma distribution $p_{A-B}(t)$ of the form:

$$
p_{A-B}(t)=\frac{k^{N} t^{N-1}}{\Gamma(N)} \exp (-k t)
$$

with $N$ the number of transitions, $k$ the rate constant, $t$ the time and $\Gamma(N)$ the gamma function [112]. This equation assumes a single rate constant $k$ for all intermediate steps, which is a reasonable assumption, as only the slowest step in a reaction significantly contributes to $p_{A-B}(t)[112$. The docking time histograms were modeled with equation 6.4 assuming a number of $N=1-4$ steps (Fig. 6.19). To estimate the quality of the modeled data, reduced $\chi^{2}$ values were derived from the fits. One finds the lowest $\chi^{2}$ values for $N=2$ transitions (green curve) with rate constants of $k=0.06 \mathrm{~s}^{-1}$ for the $90 \mathrm{~nm}$ vesicles and $k=0.08 \mathrm{~s}^{-1}$ for the $240 \mathrm{~nm}$ vesicles. The docking time distribution obtained on the f-PSM cannot be modeled due to the low number of detected events 
(Fig. 6.16C). Yet, the obtained docking times are in the same range as those obtained on the s-PSM and exhibit a similar distribution.
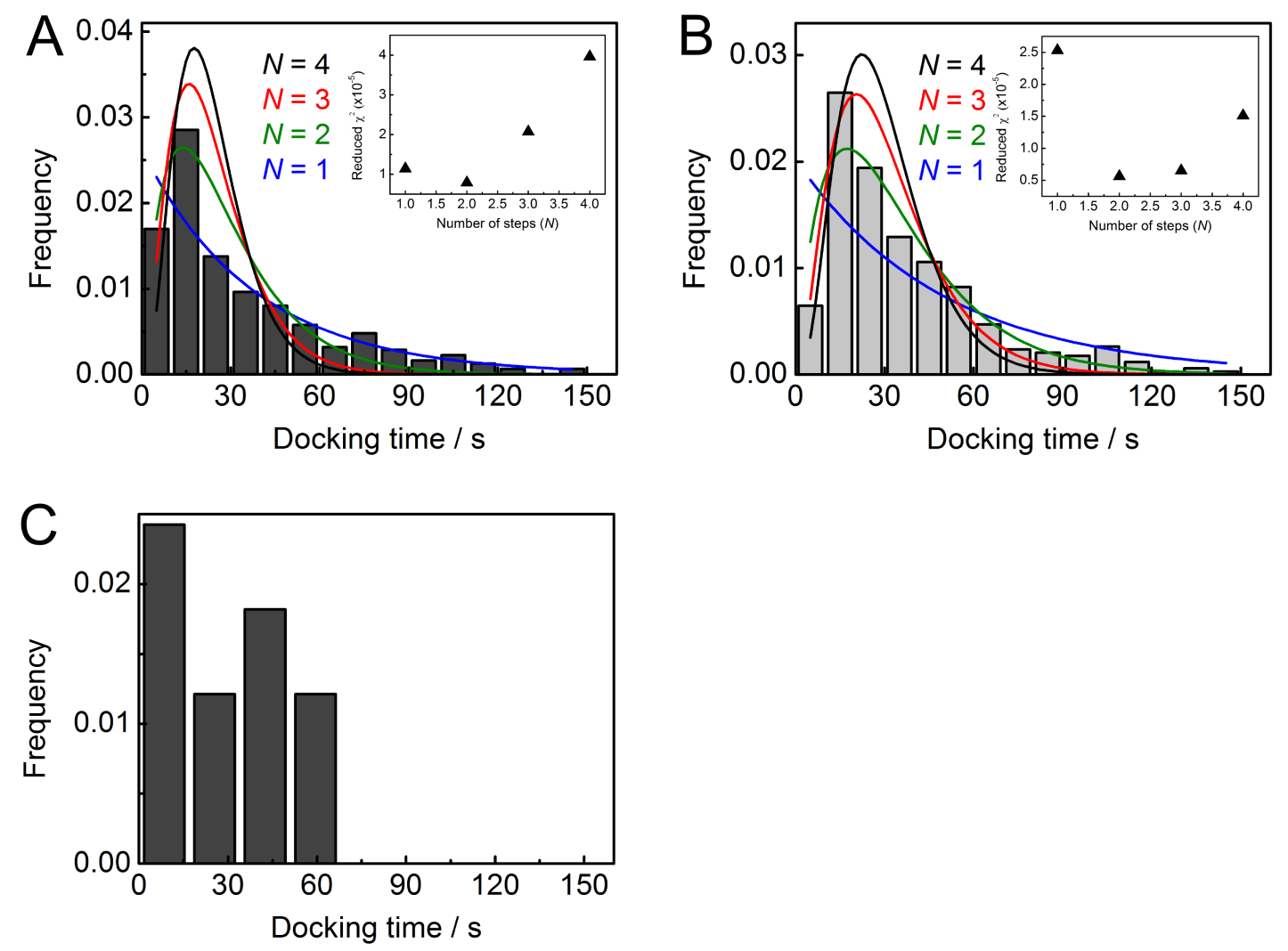

Figure 6.19. Docking time distribution of $240 \mathrm{~nm}$ vesicles fusing with the s-PSM (A) $(N=312)$ and f-PSM $(\mathrm{C})(N=11)$ and of $90 \mathrm{~nm}$ vesicles fusing with the s-PSM (B) $(N=340)$. Equation 6.4 with $N=1-4$ was fitted to the histograms shown in A and $\mathrm{B}$ to determine the number of hidden reaction steps. The insets depict the reduced $\chi^{2}$ values derived from the different fits. The bin sizes are $10 \mathrm{~s}$ in $\mathrm{A}$ and $\mathrm{B}$ and $16 \mathrm{~s}$ in $\mathrm{C}$. 


\section{Discussion: SNARE-mediated fusion on pore-spanning membranes}

The goal of this work was to extend the SNARE-mediated single vesicle fusion assay based on planar pore-spanning membranes (PSMs), recently introduced by Schwenen et al., to obtain a detailed insight into the kinetics of SNARE fusion on supported and free standing target membranes [56]. In a first step, SNARE reconstitution into large $240 \mathrm{~nm}$ vesicles and GUVs was quantified and their functionality verified by bulk experiments. In a second step, a single vesicle fusion assays was employed to analyze docking and fusion of small and large syb 2 liposomes on supported and free-standing PSMs, containing the t-SNARE $\Delta \mathrm{N}$-complex, with $10 \mathrm{~ms}$ time resolution.

\subsection{SNARE reconstitution in vesicles and pore-spanning membranes}

A stabilized t-SNARE acceptor complex, called $\Delta \mathrm{N}$-complex, and the full length vSNARE synaptobrevin 2 were used as a minimal fusion machinery. The reconstitution of syb 2 and $\Delta \mathrm{N}$-complex into liposomes was performed as described by Schwenen et al. using a lipid mixture of DOPC/POPE/POPS/cholesterol (50:20:10:20), which is comparable to the lipid composition found in synaptic vesicles and also proved to be fusogenic [56, 65]. The protocol was based on the preparation of mixed lipid-proteindetergent and lipid-detergent micelles, called mixed micelles, which were subjected to size exclusion chromatography to remove the detergent. This procedure results in spontaneous formation of small proteo-liposomes [113]. The use of the mild detergent $n$-octylglycosid ensures that proteins are not denatured and due to its high critical micellar concentration (cmc) of $20-25 \mathrm{mM}$ it can be easily removed from the mixed 
micelle solution [28]. The proteins were reconstituted with an initial protein to lipid (p:l) ratio of 1:500, which is on the same order of magnitude as in the plasma membrane of secretory cells [101]. Density gradient and SDS-PAGE analysis of syb 2 reconstitution into $240 \mathrm{~nm}$ vesicles revealed a final p:l ratio of 1:600 illustrating a nearly quantitative insertion of protein. The successful reconstitution of $\Delta \mathrm{N}$-complex into GUVs was ensured by widefield fluorescence microscopy using a fluorescently labeled Atto488- $\Delta$ N-complex. Yet it was found that only less than $1 \%$ of the initially introduced protein ended up in the GUVs. Besides the labeled GUVs, also very bright aggregates could be identified in the GUV suspensions resulting from unreconstituted labeled t-SNARE (see Chapter 6.1). The large amounts of aggregated t-SNAREs could explain the rather poor reconstitution efficiency. The protein aggregates might emerge from the drying process of the proteo-liposome suspension on the ITO slides or due to the applied electric field upon electroformation eventually denaturating the proteins.

Alternative reconstitution methods for the insertion of proteins into GUVs exist that circumvent the requirement of lipid-protein film drying and subsequent electroformation. In some of the methods small proteo-liposomes are spontaneously fused with preformed GUVs while in others preformed GUVs are destabilized with detergents to promote the insertion of solubilized proteins [114, 115]. In all of these methods the proteins are mainly reconstituted with a preferred orientation into the GUVs with their cytosolic parts facing to the outside [114]. However, this is not beneficial for the preparation of PSMs, as GUV spreading on porous substrates involves an inside out mechanism, which could be proven by high speed spinning disc confocal microscopy in this work (Fig. 7.1) and was also predicted by coarse grain simulation [116]. The adhered GUV spontaneously ruptures, starting from a defect point located at the adhesion annulus ( $t=40 \mathrm{~ms}$ ) with a receding top mechanism. This results in a planar membrane, where the majority of the initially outwardly orientated proteins now face the porous substrate and are not available for fusion anymore. The GUVs prepared in this work, however have a bidirectional orientation of the t-SNAREs ( $50 \%$ outwards) due to the drying and rehydration process, resulting in a larger fraction of t-SNAREs pointing out of the PSMs after spreading. Thus, the mentioned reconstitution methods do not represent an alternative reconstitution strategy for the preparation of PSMs.

Syb 2 and $\Delta \mathrm{N}$-complex were functionally reconstituted into LUVs and GUVs, which were prospectively used for the single vesicle asssay. The observed p:l ratios of 1:600 in the syb 2 LUVs represented a high SNARE density, which is comparable to synaptic vesicles, which exhibit a p:l ratio of roughly 1:400 [24, 117]. The GUVs, used to 

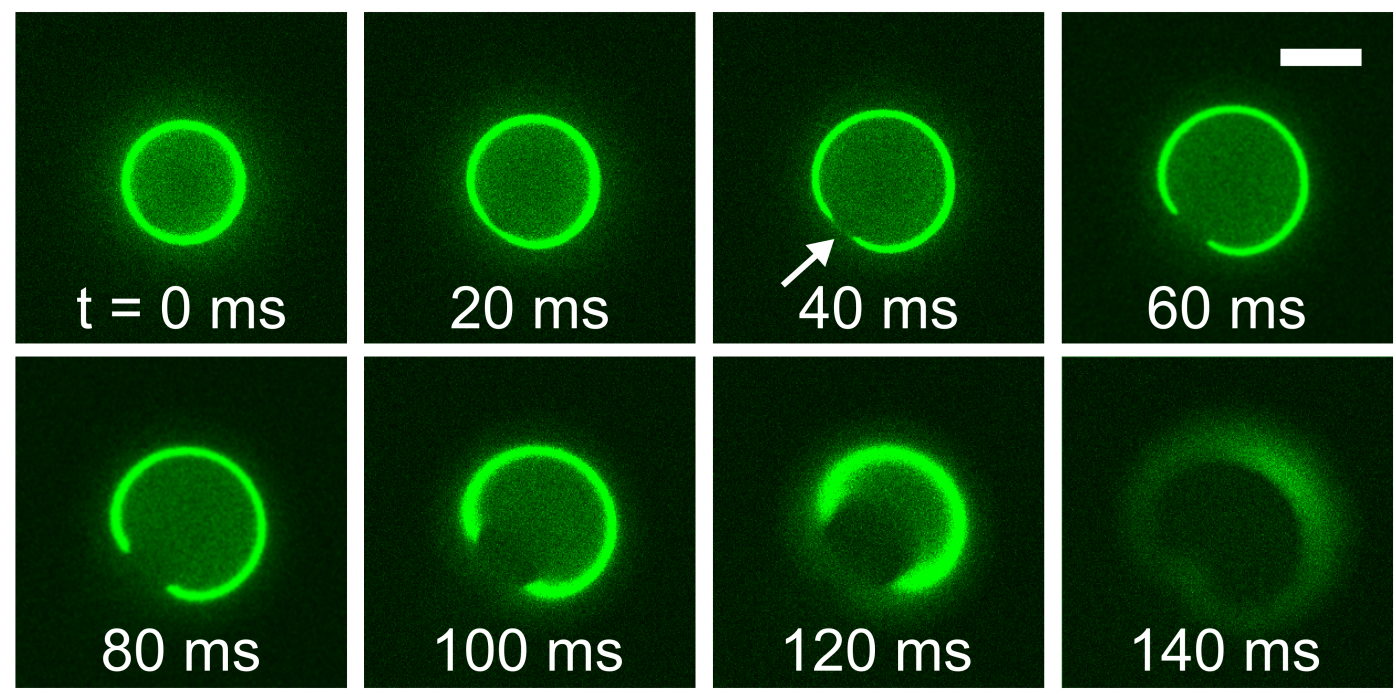

Figure 7.1. Fluorescence micrograph sequence of a GUV spreading on a porous substrate. The underlying support cannot be observed as the confocal plane was aligned above the support. Spreading of the GUV starts with a defect on the adhesion annulus (40 ms) that enlarges, resulting in a receding top mechanism. The former outer membrane leaflet of the GUVs now faces the porous support. Scale bar:10 $\mu \mathrm{m}$.

prepare PSMs, contained a significantly lower $\Delta \mathrm{N}$-complex density with an estimated p:l ratio of 1:60000. Unfortunately, estimates of t-SNARE densities in synapses are not reported in literature making it difficult to compare the obtained results. Yet, docking and fusion between LUVs and GUVs could be verified in bulk experiments, underlining that a sufficient number of t-SNAREs was reconstituted into the GUVs.

\subsection{Mobility of lipids and SNAREs in pore-spanning membranes}

The plasma membrane of mammalian cells is complex and highly dynamic, involving laterally mobile lipids and proteins within the plane of the membrane. The lateral mobility of proteins can be an important determinant of protein-binding interactions and local membrane mechanics [118]. In neuronal exocytosis, the formation of an active trans-SNARE complex, called SNARE-pin, involves the assembly of syx $1 \mathrm{~A}$ and syb 2, which are anchored in opposing membranes via their $C$-terminal transmembrane domain and SNAP 25, which is tethered to the target-membrane via several cysteinelinked palmitoyl chains. Thus, the complex formation requires a certain mobility of the 
individual proteins. To investigate the individual protein mobilities, the diffusivity of SNAREs in PSMs was analyzed in more detail. Schwenen et al. recently measured the mobility of a fluorescently labeled lipid Atto488-DPPE and a transmembrane peptide Atto647N-syx 1A-TMD, which is identical with the part that anchors the $\Delta \mathrm{N}$-complex in the target-membrane, in the f-PSM with fluorescence correlation spectroscopy (FCS) [56]. A diffusion coefficient of $D=3.4 \mu \mathrm{m}^{2} \mathrm{~s}^{-1}$ was measured for the syx $1 \mathrm{~A}$ transmembrane domain. The lipid Atto488-DPPE yielded a diffusion coefficient of $D=7.7 \mu \mathrm{m}^{2} \mathrm{~s}^{-1}$. The obtained values are comparable to mobilities measured in freestanding membranes of GUVs and black lipid membranes underlining that f-PSMs represent an adequate model system of a free-standing membrane [102, 103, 119]. They also tested to measure the mobility on the s-PSM, but the obtained fluorescence intensity was not sufficient due to the gold-induced fluorescence quenching [56].

To measure the mobilities on the s-PSM, indirect fluorescence recovery after photobleaching (FRAP) experiments were conducted in this work. This method measures the diffusion on the s-PSM indirectly by observing the fluorescence recovery from the s-PSM into the f-PSM. The obtained apparent recovery curves were modeled by finite element simulations to consider the influence of the faster diffusing f-PSM on the recovery process yielding absolute diffusion coefficients (see Chapter 6.3). For a good comparison with the values obtained on the f-PSM, the same lipid Atto488-DPPE and transmembrane peptide syx 1A-TMD was used for the experiments. It was found that the lipid Atto488-DPPE diffuses with a mean diffusion coefficient of $3 \pm 1 \mu \mathrm{m}^{2} \mathrm{~s}^{-1}$ while the syx 1A-TMD diffuses with a mean diffusion coefficient of $1 \pm 0.5 \mu \mathrm{m}^{2} \mathrm{~s}^{-1}$ in the s-PSM. Compared to the mobility in the f-PSM, the diffusivity of Atto488-DPPE and the syx $1 \mathrm{~A}-\mathrm{TMD}$ in the s-PSM is $2-3$ times slower. This is very reasonable, as diffusion coefficients obtained from solid supported membranes on $\mathrm{SiO}_{2}$ and polymercushions are also significantly lower than those in free-standing membranes [5, 48, 107]. The reduction in diffusivity is most likely a result of membrane-surface interactions that do not occur in free-standing membranes. The diffusion coefficient of the syx 1A-TMD was found to be three times smaller than that of the lipid DPPE, as also obtained in the f-PSM by Schwenen et al. These findings can be explained by a larger effective area of the TMD peptide, and the fact that the TMD is embedded in both, the upper and the lower leaflet of the membrane.

Several studies also analyzed the mobile fraction of lipids and SNAREs in solid supported membranes (SSMs) based on $\mathrm{SiO}_{2}$ or polymer-cushioned surfaces. SSMs prepared by spreading t-SNARE containing liposomes on a lipid monolayer deposited 
on polymer-cushions (85\% of the t-SNARE oriented the right way up) showed mobile t-SNARE fractions of $80 \%$ [53]. SSMs prepared by direct spreading of t-SNARE containing liposomes on $\mathrm{SiO}_{2}$ ( $50 \%$ of the t-SNARE oriented the right way up) exhibited a mobile fraction of only 3-7\% [48]. The percentage of correctly oriented proteins in the polymer-cushioned membranes approximately resembled the relative fraction of mobile protein. One can infer from this that only inversely orientated proteins are immobile in this system. Interestingly, in the membrane prepared on $\mathrm{SiO}_{2}$ the mobile fraction is much lower than expected from the $50 \%$ inversely orientated t-SNAREs. Two possible explanations can be given for this observation that might also directly correlate with each other: 1 . In comparison to the polymer-cushioned membrane, the membrane on $\mathrm{SiO}_{2}$ is not decoupled from the support. Only a thin 1-2 nm water layer separates the membrane from the support introducing strong Van-der-Waals, interaction, which might also lead to the immobilization of correctly reconstituted proteins. 2. In the $\mathrm{SiO}_{2}$ system, the t-SNARE in the SSMs consists of SNAP 25 and syntaxin 1A. This complex could build clusters via the syntaxin 1A SNARE motif, as described for pure syntaxin $1 \mathrm{~A}$ bilayers [120]. For the system on the polymer-cushion by contrast, the same stabilized t-SNARE $\Delta$ N-complex as in this work was used, where the syntaxin 1A SNARE motif is partly occupied by the small syb 2 fragment (residues 49-96). The syb 2 fragment might reduce the formation of protein clusters and therefore the number of immobile protein in the target-membrane. The diffusion coefficients obtained for the mobile t-SNAREs in the $\mathrm{SiO}_{2}$-supported membranes are also 10 times slower than those obtained in the polymer-cushioned systems and on the s-PSM, supporting the hypothesis that membrane protein interactions are more dominant [48]. Unfortunately, the immobile fraction of t-SNAREs cannot be directly determined on the s-PSM, as only mobile protein can recover into the f-PSM (see Chapter 6.3). Therefore, a direct comparison with the other assays is not possible. Yet it is assumed that at least $50 \%$ immobile fraction are present, as the $\Delta \mathrm{N}$-complex is expected to be equally and bidirectionally distributed in the GUVs resulting in PSM patches with $50 \%$ of the t-SNARE oriented the right way up and $50 \%$ facing the support. Table 7.1 gives an overview of reported diffusion coefficients and immobile fractions of lipids and SNAREs in the various single vesicle fusion assays.

Indirect FRAP combined with finite element simulations could be established in this work to quantify the mobility of lipids and proteins in the s-PSM, which are indeterminable by methods like normal FRAP or FCS. It was found that lipids and a syx $1 \mathrm{~A}-\mathrm{TMD}$ are mobile in the s-PSM with diffusion coefficients of $3 \mu \mathrm{m}^{2} \mathrm{~s}^{-1}$ and 
Table 7.1. Diffusion coefficients of lipids and SNAREs in planar membranes and free standing membranes prepared on different supports.

\begin{tabular}{ccccc}
\hline Support & Label & Technique & $\begin{array}{c}\text { Diff. Coeff. } \\
\mu \mathrm{m}^{2} \mathrm{~s}^{-1}\end{array}$ & $\begin{array}{c}\text { Mobile fraction } \\
\%\end{array}$ \\
\hline $\mathrm{SiO}_{2}$ & t-SNARE & FRAP & $0.9 \pm 0.1$ & $83 \pm 2$ \\
{$[48$, 107] } & syx 1A & FRAP & 0.07 & $3-7$ \\
& eggPE & FRAP & $1.3 \pm 0.1$ & $85 \pm 1$ \\
& eggPC & FRAP+SPT & $1-5$ & $85 \pm 1$ \\
& & & & $78 \pm 3$ \\
PEG & t-SNARE & FRAP & $0.75 \pm 0.2$ & $92 \pm 7$ \\
{$[107]$} & eggPE & FRAP & $1.0 \pm 0.1$ & - \\
SAM & syx 1A-TMD & ind. FRAP & $1.0 \pm 0.3$ & - \\
(s-PSM) & DPPE & ind. FRAP & $3.0 \pm 0.5$ & - \\
free standing & syx 1A-TMD & FCS & $3.4 \pm 0.2$ & - \\
(f-PSM) [56] & Atto488 DPPE & FCS & $7.7 \pm 0.4$ & \\
\hline
\end{tabular}

$1 \mu \mathrm{m}^{2} \mathrm{~s}^{-1}$, respectively. The observed diffusion coefficients are in good agreement with those obtained on polymer-cushioned and glass supported membranes and underline the high mobility of the membrane components, which are prerequisite for SNARE assembly and the dynamic fusion process [48, 53, 117].

\subsection{SNARE-mediated single vesicle fusion assay}

To gather informations about fusion products and compare fusion kinetics, SNAREmediated fusion of single vesicles with PSMs was investigated with high speed spinning disc microscopy. Docking and fusion of syb 2 vesicles with different diameters on the supported and free-standing membranes was analyzed in detail to study the impact of vesicle size on fusion kinetics.

\subsubsection{Vesicle docking to pore-spanning membranes}

Syb 2 vesicles were prepared by extrusion of multilamellar syb 2 liposomes through polycarbonate membranes with nominal pore diameters of $50 \mathrm{~nm}$ or $400 \mathrm{~nm}$, respectively. The size distribution of the prepared vesicles was analyzed by dynamic light scattering (DLS). Extrusion through $50 \mathrm{~nm}$ membranes yielded vesicles with a rather 
narrow size distribution with a mean diameter of $90 \pm 40 \mathrm{~nm}$. Extrusion through $400 \mathrm{~nm}$ membranes yielded a much broader size distribution of the vesicle population with a mean diameter of $240 \pm 100 \mathrm{~nm}$. The obtained vesicle sizes are in good agreement with the sizes of protein free eggPC vesicles prepared by a similar extrusion technique and analyzed by freeze-fracture electron microscopy [121]. Vesicle diameters of $243 \pm 93 \mathrm{~nm}$ and $68 \pm 19 \mathrm{~nm}$ were observed after extrusion through $400 \mathrm{~nm}$ and $50 \mathrm{~nm}$ membranes, respectively. The slightly larger diameters of the vesicles obtained by extrusion through $50 \mathrm{~nm}$ membranes in this work can be explained by the different lipid mixture. While Mayer et al. used pure eggPC, in this work a mixture of DOPC/POPS/POPE/cholesterol (5:1:2:2) was used containing lipids striving for a larger negative spontaneous curvature (POPE and cholesterol) [122].

For the single vesicle experiments, GUVs containing the t-SNARE $\Delta \mathrm{N}$-complex were spread on porous substrates functionalized with a SAM composed of 6-mercaptohexanol (6-MH) resulting in PSMs. Successful PSM formation was monitored with fluorescence microscopy prior to each experiment. Image acquisition was started and vesicles containing syb 2 were injected over a PSM patch, which then randomly docked to the planar target-membrane. To ensure that vesicle docking is SNARE dependent control experiments with a blocked $\Delta \mathrm{N}$-complex were conducted, similar to those performed in the bulk SNARE activity tests, showing no docking of syb 2 vesicles to the PSM. This proves docking as a fully SNARE dependent process. Experiments from the former co-worker Lando Schwenen also proved that fusion on PSMs is SNAP 25 dependent as observed by other groups on PEG-supported membranes [53, 98]. In contrast to these observations fusion on $\mathrm{SiO}_{2}$-supported membranes was frequently reported to be independent of SNAP 25 [48, 50]. It is possible that the PEG layer and the self-assembled monolayer in the system used in this work, efficiently decouple the target-membrane from the support, which significantly reduces the lateral tension as well as defects in the membrane thus preventing non-specific fusion.

Docking of syb 2 vesicles took place on both, s-PSMs and f-PSMs. Considering the different area sizes of the s-PSM (64\% of the total surface area) and the f-PSM (36\% of the total surface area) it was found that the probability of a vesicle to dock to the s-PSM is two times larger than to dock to the f-PSM. This finding might be a result of different protein concentrations in the s-PSM and the f-PSM, e.g. a protein depletion in the f-PSM. This would lead to different docking probabilities on the two membranes. Yet, indirect FRAP experiments proved free diffusion of lipids and a syx 1A-TMD between the two membrane sites, which would compensate a protein gradient. This 
however, does not consider immobile t-SNAREs, which are frequently described in supported membranes and are also expected in the s-PSMs, as discussed in detail in Chapter 6.3. Immobile protein could lead to a higher protein concentration on the s-PSM increasing the docking probability of the syb 2 vesicles. Immobile t-SNAREs might also be more efficient docking sites than mobile t-SNAREs, as suggested by Liu et al. [50].

Vesicles docked to the s-PSM were found to be completely immobile (see Chapter 6.4.3). Indeed, all published single vesicle fusion systems based on supported planar membranes, report immobile SNARE-docked vesicles [48 51, 53, 98]. Although large deviations between these systems exist, e.g. SNAP 25 dependency and mobility of tSNAREs, significant amounts of immobile t-SNAREs remain, which might be sufficient to effectively immobilize docked vesicles. Even though the amount of immobile t-SNAREs cannot be directly determined in the PSM, owing to the gold-induced fluorescence quenching on the pore rims, it is well conceivable that a significant fraction of the $\Delta \mathrm{N}$-complex is also immobile in the s-PSMs resulting in immobile docked vesicles on this membrane part.

Membrane defects in the target-membrane are also discussed in literature as possible immobilization points and artificial fusion sites for the docked vesicles [53]. Membrane defects can be an issue in solid supported membranes prepared by small vesicles. The small vesicles spread on the solid support and merge to an interconnected bilayer [123. During this process, small membrane defects can occur, which are too small to be identified by fluorescence microscopy [124]. Yet, such defects should not be an issue in PSMs as these are prepared by spreading an entire defect free GUV over the porous support. This assumption is supported by the fact that unspecific binding, e.g. protein-defect interactions, of syb 2 vesicles to PSMs containing a blocked $\Delta \mathrm{N}$-complex (control experiments) could never be observed. It can however not be fully ruled out that membrane defects emerge over time on the s-PSM.

In contrast to s-PSM-docked vesicles, vesicles docked to the f-PSM are fully mobile with a mean diffusion coefficient of $0.42 \mu \mathrm{m}^{2} \mathrm{~s}^{-1}$. These results are very valuable as syb 2 vesicles docked to large, planar free standing target-membranes could not be observed until now. The mobility of the vesicles underlines the hypothesis that immobile protein, which does not exist in f-PSMs, plays a crucial role in the observed immobilization of docked vesicles. Furthermore, the free standing membranes appear to be the more physiological system compared to the SSMs, as synaptic vesicles docked to the pre-synaptic membrane are also laterally mobile with diffusion coefficients of 
$D=5 \cdot 10^{-5} \mu \mathrm{m}^{2} \mathrm{~s}^{-1}[125]$. The significantly smaller diffusion coefficients of the synaptic vesicles compared to the f-PSM-docked vesicles are most probably a result of the smaller cage size $(50 \mathrm{~nm})$ and the much higher protein density within the targetmembrane. These findings demonstrate that mobile SNAREs are per se sufficient to dock and fuse membranes. Interestingly, the obtained diffusion coefficients of the f-PSM docked vesicles are by a factor of 8 lower than the diffusivity of a single syx 1A-TMD, which represents the part of the t-SNARE anchoring the vesicle to the membrane. A possible explanation for this observation might be the number of SNARE contacts between the vesicle and the target membrane. Multiple tethers between vesicles and planar membranes reduce the mobility of the vesicles easily by a factor of 3-5 compared to a single tether [126, 127]. Multiple SNARE complexes formed between the vesicle and the f-PSM could therefore explain the slower diffusivity. Several other groups could also experimentally verify that multiple SNARE-complexes are involved in docking and fusion of vesicles [32, 53, 128-130]. Also frictional coupling between the two membranes, as a result of the tight SNARE zippering, could reduce the lateral mobility of the vesicles significantly.

The f-PSM docked vesicles exhibit confined diffusion inside the pore. This points towards a barrier at the pore rim, which only affects the vesicle diffusion, while lipids and proteins can freely diffuse over this barrier (see Chapter 6.3). Analysis of the radial probability density of vesicles docked to the f-PSM revealed that the probability to find a vesicle in the pore center is higher than to find it on the pore rim, underlining the existence of such a barrier. Approaching this barrier results in an increasing energy penalty for the vesicle. As the velocity and thus the diffusivity of the vesicles remain constant within the entire f-PSM, the energy barrier though does not affect the vesicle's mobility. A possible explanation for this observation could be the membrane topology on the pore rims. AFM and SICM studies revealed that PSMs are not flat, but exhibit a height difference between the s-PSM and the f-PSM [131]. This height difference is most probably a result of the membrane partially following the curvature of the pore rim before it starts to freely span the pore as indicated schematically in Figure 7.2. Assuming a more or less planar contact zone between the docked vesicles and the target-membrane, deformation of this zone at the pore rim would lead to an increasing energy penalty when the vesicle approaches this region. Yet, the probability to find a vesicle at the pore rim is not zero, explaining the large amounts of immobilized vesicles on the pore rim over time. In fact, $89 \%$ of the initially f-PSM docked vesicles are immobilized on the pore rim before fusion occurs. It is assumed 
that the same mechanism, e.g. immobile t-SNAREs located around the f-PSM, leads to the immobilization on the pore rim. Once immobilized on the rim, most vesicles were not able to regain their mobility, which is comparable to the effect of the very rarely observed detachment of s-PSM docked vesicles.

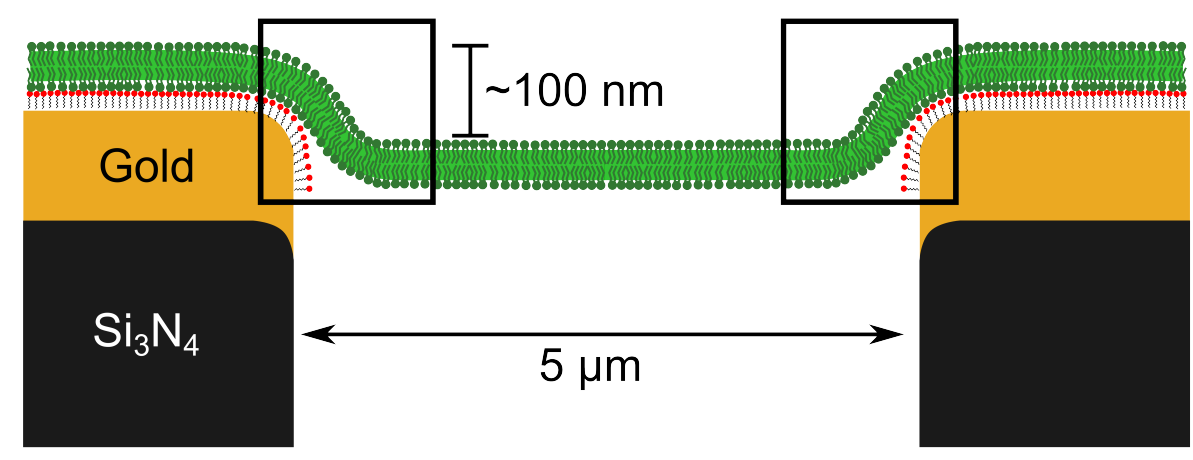

Figure 7.2. Schematic drawing of a single pore-spanning membrane. The membrane partly follows the edges on the pore rim before it starts to freely span the pore. The height difference between the free standing and the supported membrane leads to a membrane curvature at the pore rim, marked by the black boxes.

Docking of syb 2 vesicles to $\Delta \mathrm{N}$-complex PSMs could be observed on a single vesicle level with a time resolution of $10 \mathrm{~ms}$. It was found that the vesicles have a $50 \%$ lower probability to dock on the f-PSM than to dock on the s-PSM, which is explained with immobile t-SNAREs acting as efficient docking sites on the s-PSM. s-PSM docked vesicles were fully immobile once docked to the membrane, which is in good agreement with results from studies using solid supported membranes on glass or polymer-cushions [48 50, 53, 108]. By contrast, vesicles docked to the f-PSM revealed a high lateral mobility inside the f-PSM with a mean diffusion coefficient of $0.42 \mu \mathrm{m}^{2} \mathrm{~s}^{-1}$. Mobile, SNARE-docked vesicles, on a completely free-standing planar membrane could not be observed until now. This observation proves for the first time that SNARE-docking is per se independent of immobile t-SNARE clusters in planar membranes as hypothesized before [48]. As synaptic vesicles docked at the presynaptic membrane are also mobile, f-PSMs offer the clear advantage of high dynamics, which cannot be found in other in-vitro fusion assays. The lower diffusivity of the docked vesicles compared to a single SNARE component is explained with a tight contact zone between the vesicle and the planar membrane and provides evidence for multiple SNARE-complexes. 


\subsubsection{Interpretation of fusion events}

Fusion of docked syb 2 vesicles with $\Delta \mathrm{N}$-complex doped PSMs was investigated by analyzing the TR intensity in a ROI located at the center of each vesicle. Upon fusion, TR diffuses out of the vesicle, resulting in the observed fluorescence decay. It could be verified that the TR decay is diffusion controlled by applying a lipid disc source model for events taking place on the f-PSM (see Chapter 6.4.4). The successful lipid mixing could also be verified around the fusing vesicle and analyzing the TR intensity distributing around the vesicle in the planar target membrane. The TR decay can therefore be seen as a reliable indicator for lipid mixing triggered by fusion. It is important to note that this analysis does not work for events taking place on the s-PSM due to the gold-induced and height dependent fluorescence quenching (see Chapter 6.4.4). Most of the observed fusion events of $90 \mathrm{~nm}$ and $240 \mathrm{~nm}$ vesicles with the s-PSM showed a full TR intensity decay back to the pre-docking intensity (Tab. 7.1). These events were classified as full fusion, as no intermediate states with a lifetime longer than the time resolution of the measurement $(10 \mathrm{~ms})$ could be observed (Fig. 7.3). All fusion events observed on the f-PSM could be identified as full fusion events.

Besides the full fusion events, a fraction of the events showed intermediate TR fluorescence intensities between the pre-docking and docking intensity after the onset of fusion, which eventually recovered to the pre-docking level in a second step (Fig. 7.3). The percentage of full fusion and intermediate fusion events is virtually identical for $90 \mathrm{~nm}$ and $240 \mathrm{~nm}$ vesicles. In the following, it will be discussed in detail how these temporary intermediate TR intensities can be explained.

Table 7.2. Percentage distribution of full fusion and intermediate fusion events obtained for vesicles with a diameter of $90 \mathrm{~nm}(N=340)$ and for vesicles with a diameter of $240 \mathrm{~nm}(N=312)$.

\begin{tabular}{cccc}
\hline $\begin{array}{c}\text { Mean vesicle } \\
\text { diameter } \\
\text { nm }\end{array}$ & $\begin{array}{c}\text { Full } \\
\text { Fusion } \\
\%\end{array}$ & $\begin{array}{c}\text { Intermediate } \\
\text { Fusion } \\
\%\end{array}$ & $\begin{array}{c}\text { Intermediate } \\
\text { to Full Fusion } \\
\%\end{array}$ \\
\hline 90 & 86 & 14 & 5 \\
240 & 87 & 13 & 9 \\
\hline
\end{tabular}

Many groups have reported characteristic fusion intermediates that could be identified 


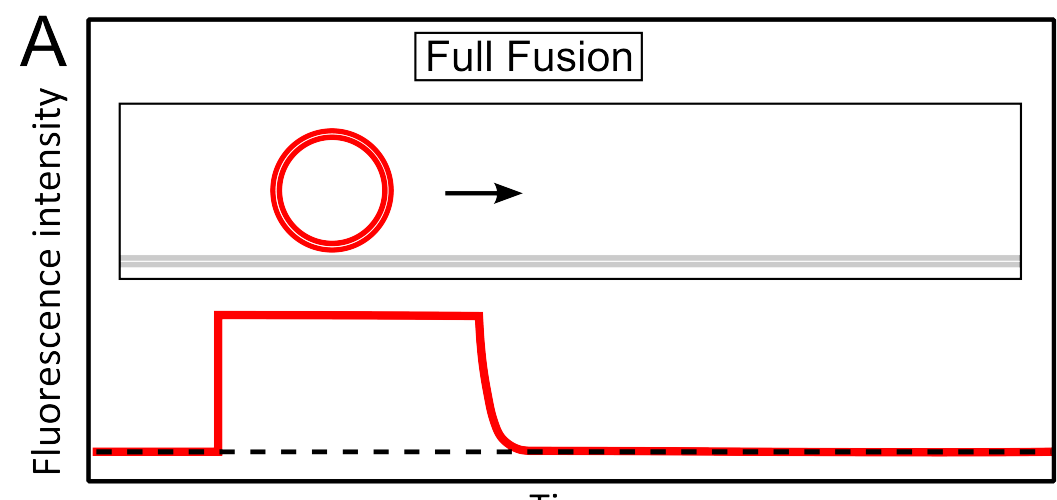

Time

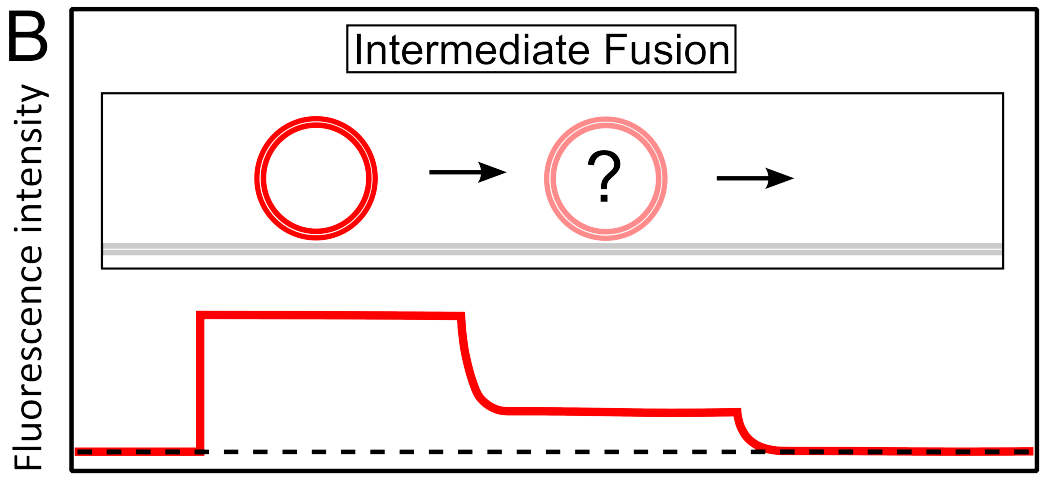

Time

Figure 7.3. Schematic drawing and expected time resolved TR intensity trace of a full fusion event (A) and an intermediate fusion events (B). In case of the full fusion event, the TR intensity recovers to the pre-docking intensity (---) in a single step. In case of the intermediate fusion event, the TR intensity drops to an intensity level between docking and pre-docking, which eventually recovers to the pre-docking intensity in a second step. The semi-stable intermediate TR intensity could be a result of fusion intermediates or residual TR intensities from vesicle impurities.

via fluorescence microscopy and double patch clamp techniques [32, 45, [54, 132 137]. Hemi fusion is one of the most frequently reported fusion intermediates. In hemi fusion, only the proximal outer leaflet of the vesicle membrane has fused with the targetmembrane, while the distal inner leaflet remains intact (Fig. 7.4). An enclosed vesicle cargo cannot be released in this state. Only when the inner vesicle leaflet also fuses with the target membrane, resulting in a fully fused vesicle, the cargo will be released. Hemi fusion was observed in several in-vitro model systems [32, 45, 54, 132, 135, 137] and is predicted from simulations for fusion of protein free bilayers [138]. Recently, Zhao et al. also made the first observations of hemi-fused structures in live cells, indicating that hemi fusion is not only a feature of synthetic in-vitro systems as assumed for a long time [139]. On PSMs, hemi fusion intermediates could be identified by the TR 
intensity decrease of the labeled vesicle membrane. As only the outer membrane leaflet of the vesicle fuses with the target-membrane in hemi fusion, a TR intensity decrease to around $50 \%$ of the docking intensity should be observable. The gold-induced and height dependent quenching is not expected to significantly alter the TR intensities, as the size and the shape of the hemi fused vesicle remains the same. A very similar analysis was performed by Lengerich et al. to characterize hemi fusion intermediates during fusion of fluorescently labeled vesicles mediated by lipid-anchored DNA on solid supported membranes [137]. They found intensity levels around $50 \%$ for hemi fused vesicles. Compared to the intermediate intensity levels observed on the f-PSM in this work, only a very few events with a relative TR intensity between around $50 \%$ were found (see Chapter 6.4.6). Most of the levels showed a decrease to an intensity level below $50 \%$ giving rise to the assumption that hemifusion is not the dominant fusion intermediate on the s-PSMs. It is however possible that hemifusion appears on a time scale below the $10 \mathrm{~ms}$ time resolution in the experiments. Other fusion intermediates reported in literature involve transient fusion pores that randomly close and reseal the vesicle [45, 136]. A randomly closing fusion pore would disturb full lipid mixing, leaving a certain amount of fluorophores inside the vesicle, which could explain the vastly different TR intensity levels (Fig. 7.4). Along the same line, a transient pore flickering, indicating a very fast opening and closing of the fusion pore, can be excluded as the intermediate TR intensity level remains constant in all events [140].

Besides fusion intermediates, inhomogeneities in the vesicle suspensions, e.g. lamellarity of the vesicles or vesicle inclusions could be an alternative explanation for the observed intermediate TR intensity levels. Vesicles produced by extrusion can be multilamellar or encapsulate aggregated lipid material or smaller vesicles [121, 141]. Such encapsulated smaller vesicles could be released upon fusion also subsequently fusing with the target membrane as depicted in Figure 7.5A and B. Depending on the size of the inclusion, different TR intensity levels could occur, which would be always smaller than the intensity of the vesicle host $(<50 \%)$. To further investigate if such inclusions are responsible for the observed intermediate states, the total fluorescence intensity of the docked vesicles was analyzed and plotted for vesicles showing intermediate TR intensities and those fully fusing with the membrane (see Chapter 6.4.6). The intensity distributions indicate slightly higher intensities of the vesicles with intermediate TR levels, which could originate from inclusions. Yet, a two-sample KS-Test classified the distributions as not significant prohibiting any further conclusions. It can therefore 

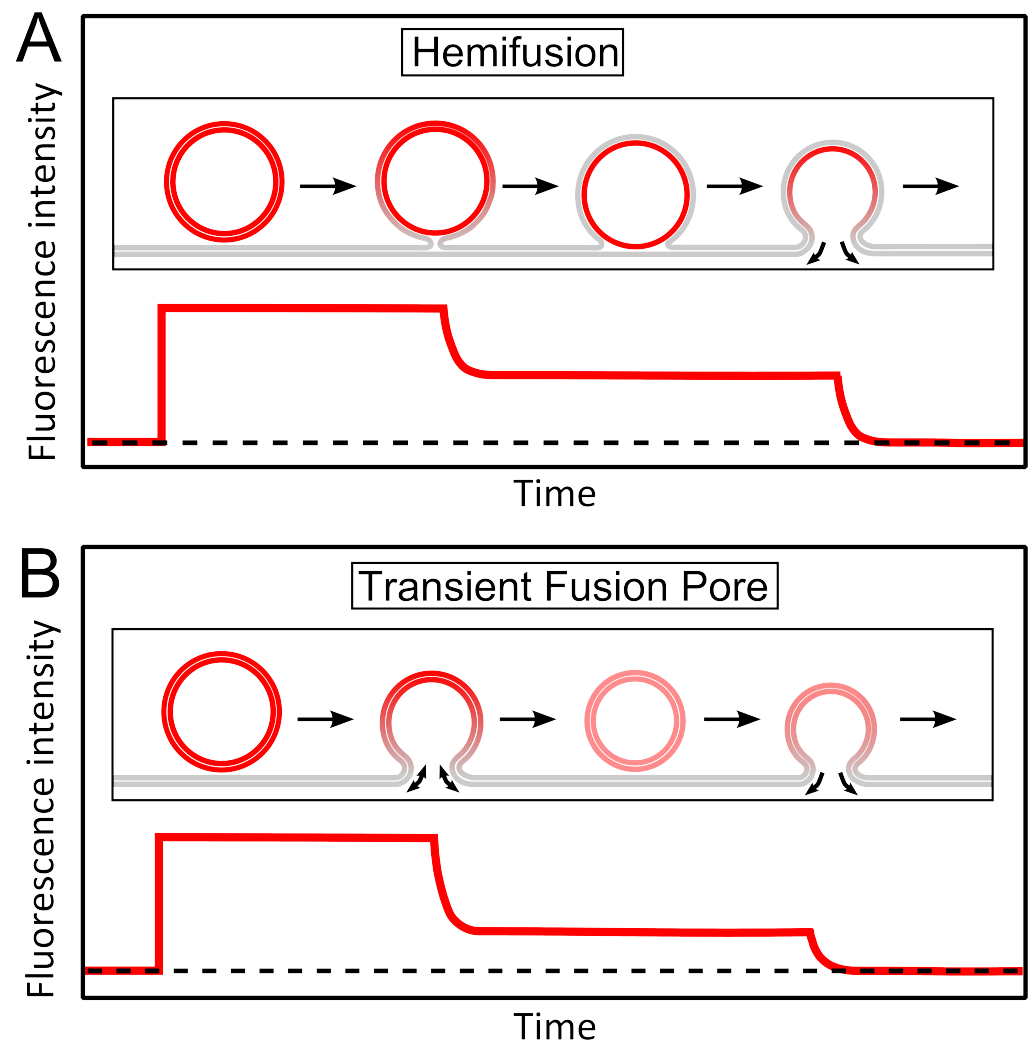

Figure 7.4. Schematic drawing of a productive hemi fusion event (A) and productive transient fusion pore event (B) and the corresponding expected time resolved TR intensity traces.

not be safely concluded if indeed lipid inclusions or actual fusion intermediates are responsible for the observed intermediate TR intensities.

Analysis of the time resolved TR intensity traces of syb 2 vesicles fusing with $\Delta \mathrm{N}$ complex PSMs showed a full TR decay in $86 \%$ of the events, which is indicative of full fusion events with no intermediates, within the $10 \mathrm{~ms}$ time resolution of the experiments. This result is supported by findings of other groups who also report large amounts of fast and full fusing vesicles in their assays [48, 53]. A detailed analysis of the remaining 13-14\% of fusion events, showing intermediate TR levels, is suggested to result from bilamellar vesicles and vesicular inclusions, commonly obtained as a byproduct during vesicle extrusion. Future experiments with content labeled vesicles could help to further ascertain whether fusion intermediates or lipid inclusions lead to the observed intermediate levels. 


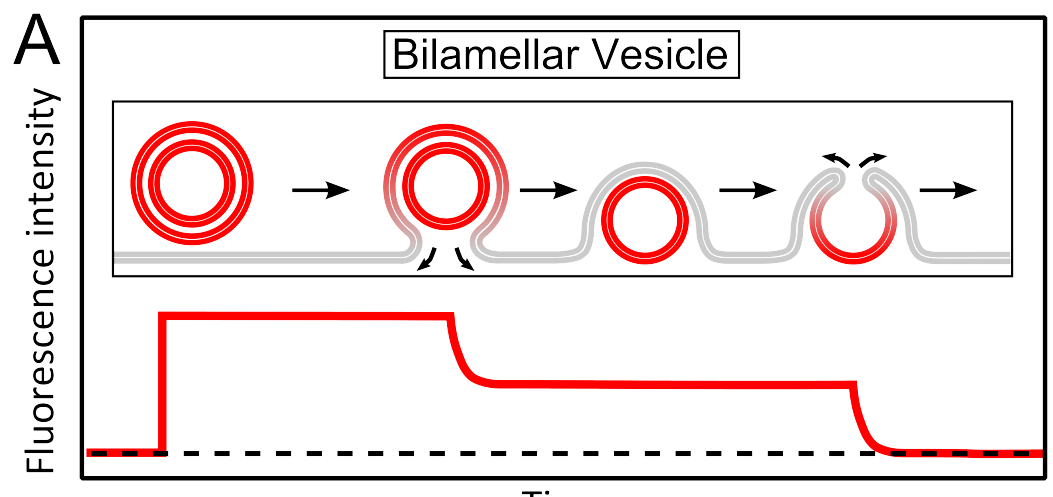

Time

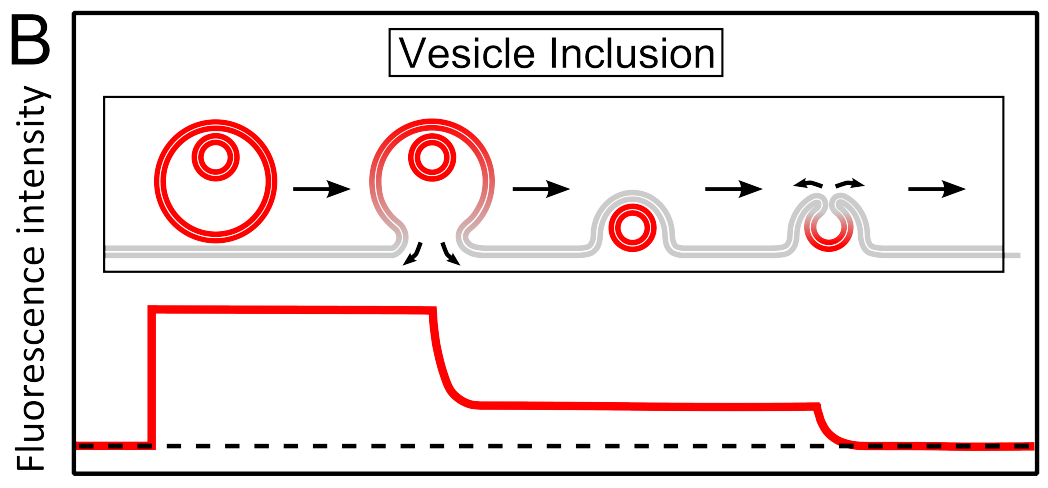

Time

Figure 7.5. Schematic drawing of a bilamellar vesicle (A) and vesicle carrying a smaller vesicle (B) fusing with the target-membrane and the corresponding expected time resolved TR intensity traces.

\subsubsection{Post fusion structures of vesicles}

Using two-color spinning disc fluorescence microscopy, dequenching of the Atto488 labeled target-membrane was successively utilized to gather informations about the post-fusion topology of fused vesicles on the s-PSM. It was found that $47 \%$ of the analyzed vesicles $(N=60)$ rapidly merged into the target-membrane, while the residual $53 \%$ merged significantly slower into the target-membrane.

Kiessling and co-workers also investigated the topology of $50 \mathrm{~nm}$ syb 2 vesicles fusing with PEG-supported target-membranes by polarized Total Internal Reflection Fluorescence Microscopy (TIRFM) and found that the vesicles immediately flattened and merged into the membrane within $8 \mathrm{~ms}$ after fusion started. These findings are in good agreement with the fast merging vesicles observed in this work. However, semi-stable post fusion structures could not be observed in the cited work. Studies from Chiang et al. and Anantharam et al. revealed $\Omega$-shaped post fusion structures upon exocytosis of 
secretory granules [110, 111]. Such structures are discussed in literature in the context of a Kiss-And-Run mechanism, where the fusion pore reseals again, retaining the vesicles integrity when it discharges its content, conserving the vesicle structure for a more efficient recycling for the next fusion cycle [142]. A controlled fusion pore closing though, must be controlled by additional proteins, which are not present in the system used in this work. An alternative explanation for the semi-stable post fusion structures might therefore be an interference of the content release. To observe full collapsing on the s-PSM, the entire vesicle content must be released in the space between the s-PSM and the SAM, which consists of an ultrathin (1-2 nm) water layer [143, 144]. To achieve this, the s-PSM must be either lifted further away from the support to incorporate the content, or the thin water layer must be displaced in an area of several $\mu \mathrm{m}^{2}$ around the fusion site. Such processes might be time consuming and therefore significantly influencing the release rate leading to the observed post-fusion structures. Events with a rapid collapsing structure might in contrast release their content above the membrane due to transient membrane defects occurring from the highly deformed vesicle membrane in the $\Omega$-like structure. This hypothesis is supported by Wang and co-workers, who studied fusion of content labeled syb 2 vesicles with planar glass supported t-SNARE membranes [51]. They found that most of the vesicular content is released above the target-membrane upon fusion rather than under the membrane due to transient membrane defects. To further quantify the observed structures on the s-PSM, vesicles filled with a fluorescently labeled content could be used according to the study of Wang et al. to precisely correlate post fusion structures with a content release.

\subsubsection{Kinetic analysis of fusion events}

The kinetics of single vesicle fusion with PSMs were analyzed by determining the time between docking and the onset of fusion, called docking time. Docking times for small $90 \mathrm{~nm}$ and larger $240 \mathrm{~nm}$ vesicles on s-PSM and on the f-PSMs were found to be on the time scale of seconds, with a maximum at 12.5 seconds and 16.7 seconds, respectively. The lifetime distributions are very similar demonstrating that neither the size of the vesicles, nor the fusion site (s-PSM vs. f-PSM) significantly alters the docking time. On a first glimpse, this is rather unexpected, as the smaller vesicles exhibit more curvature stress than the larger vesicles, which is believed to support the formation of a fusion pore and fusion pore expansion [4, 13, 36, 145, 146]. Also the pre-stressed f-PSM does not significantly influence the docking times of the vesicles compared to 
the s-PSM. These observations give rise to the assumption that the observed fusion kinetics are mainly dominated by the SNAREs rather than by mechanical parameters like membrane tension and curvature stress.

Domanska et al. analyzed fusion of small $50 \mathrm{~nm}$ syb 2 vesicles with planar PEGsupported target membranes containing t-SNAREs by means of TIRF microscopy. They found docking times on the millisecond time scale, roughly 1000 times faster than observed in this study [53]. Similar docking times were also observed in assays using glass-supported target membranes containing the t-SNAREs syntaxin 1A and SNAP 25 [51, 98, 132]. By contrast, in other fusion assays using glass-supported membranes, docking times to be on the second time scale [48, 49]. Yet, in all systems fusion was reported to be SNARE dependent. Despite the great variety in the docking times, non of the assays achieved fusion kinetics comparable to those found in stimulated synaptic boutons, which are on the sub millisecond time scale [17, 147, 148]. To explain the vastly different docking times, one has to consider the different t-SNAREs used in the assays. In most cases, syx 1A and SNAP 25 were reconstituted directly into the target-membrane to form a fusogenic 1:1 t-SNARE complex [48 51, 98]. This is a reasonable procedure, as syx $1 \mathrm{~A}$ and SNAP 25 resemble the main fusogenic t-SNARE motif in synaptic boutons. However, studies showed that the syx 1A/SNAP 25 dimer can bind a second syx 1A resulting in a very stable syx 1A/SNAP 25 2:1 complex, which is not fusogenic anymore [28, 149]. Depending on the preparation time and protein concentration in the different assays, it is feasible that significant amounts of inactive 2:1 complex were formed well before fusion was investigated. Thus, comparison of the fusion kinetics obtained from these studies is difficult. Interestingly, some of the assays showed SNAP 25 independent fusion, indicating that fusion might also be triggered by factors like temperature and membrane defects rather than a four helix SNARE bundle. Bowen et al., for example, suggest a strong temperature dependency of the fusion process in their single vesicle fusion assay. They found significantly shorter docking times when they performed the experiments at $37^{\circ} \mathrm{C}$ instead of at room temperature. However, many assays with docking times on the millisecond timescale were performed at room temperature ruling out temperature as a dominant factor explaining the differences in the docking times [50, 51, 98. Also defects in the target membrane are discussed as a possible fusion catalyst, but no direct evidence for such defects can be found in the reported systems [53, 124]. The t-SNARE concentration in the target membrane might also be an important factor affecting the overall docking time. Docking of syb 2 vesicles was found to depend on the t-SNARE density in the 
target-membrane [53]. At higher t-SNARE concentrations, docking of syb 2 vesicles was increased with a maximum efficiency at $476 \mathrm{t}$-SNAREs $/ \mu \mathrm{m}^{2}$. However, the impact of SNARE density on the fusion kinetics was not yet studied systematically.

In this work, the stabilized t-SNARE $\Delta \mathrm{N}$-complex consisting of syx $1 \mathrm{~A}$, SNAP 25a and a syb 2 fragment (residues 49-96) was used [28]. The syb 2 fragment prevents binding of a second syx $1 \mathrm{~A}$ and therefore the formation of an inactive 2:1 complex, as observed using only syx $1 \mathrm{~A}$ and SNAP 25. The v-SNARE syb 2 can bind to the unoccupied N-terminal part of the $\Delta \mathrm{N}$-complex and displace syb 2 fragment allowing full zippering of the SNARE complex. The displacement of the syb 2 fragment was measured by bulk anisotropic measurements and identified as the rate limiting step between docking and fusion with a half life of 19-20 seconds [28, 150, 151]. The displacement of the syb 2 fragment therefore perfectly explains the long docking times of the vesicles, which are also in the second regime, found in the single vesicle assay in this work. Interestingly, Domanska et al. also used the $\Delta \mathrm{N}$-complex in their single vesicle fusion assay but reported docking times in the millisecond regime, which is quite contradictory considering the slow displacement of the fragment [53]. It is feasible that other fusogenic factors like membrane defects or residual detergent from the protein reconstitution might accelerate the observed fusion kinetics in this assay.

\section{Fusion is a multiple step process}

The slow displacement of the syb 2 fragment is interpreted as the rate limiting step between docking and fusion [28, 150, 151]. One would therefore expect an exponential decay of the docking time distribution. Yet, the observed docking time distributions show a rise and decay of the frequency of events (see Chapter 6.4.7), indicating a multiple step process with a hidden number of transitions $N$. N is qualitatively encoded in the shape of the curve and can be extracted by fitting the docking times with the gamma distribution [152]. It is important to note that this model assumes identical rate constants between each step. This is a reasonable assumption because vastly different rate constants, e.g. a very fast one and much slower one, would result in a pseudo exponential dwell time distribution with the rate constant of the slowest process. Fitting the gamma distribution with $N=2$ steps to the analyzed docking time distribution of $90 \mathrm{~nm}$ and $240 \mathrm{~nm}$ vesicles, yielded the best results with rate constants of $k=0.06 \mathrm{~s}^{-1}$ and $k=0.08 \mathrm{~s}^{-1}$, respectively. The parameter $N$ resembles more or less a formal parameter due to the various simplifying assumptions and can therefore be interpreted in two ways [112, 152]. The first interpretation relates $N$ to 
a number of sequential processes, which could result from molecular transitions in the formation of a single SNARE complex or the synchronized formation of multiple complexes. Also processes like membrane deformations at the contact zone would be conceivable. Recent studies showed that SNAREs can assemble in multiple stages from the $N$-termini towards the $C$-termini cycling through a sequential, two-step folding pathway [153, 154]. This process however takes place on a time scale of less than one millisecond and therefore should not affect the docking time distribution. Cryo electron microscopy studies from Baharat et al. revealed different states of docking between small unilamellar vesicles (SUVs) and giant unilamellar vesicles (GUVs) containing SNAREs prior to fusion [155]. They could identify three different contact classes. In the first class (normal contact), a close proximity between the SUV and GUV was observed without any obvious distortion induced in the vesicle. In the second class (extended contact) a more extended membrane contact could be observed with two closely opposing membranes. In the third class (protrusion), a prominent extrusion pointing towards the SUV could be observed. Such extrusions induce a curvature in the target membrane, and are expected to support fusion [13, 156]. A vesicle docked to a target-membrane protrusion could resemble a state, which is comparable to a primed synaptic vesicles, ready for fusion [157-159]. A significant energy penalty can be expected from protruding the target membrane, as it looses adhesion energy to the support, which would make this process rather slow. However, the docking times on the f-PSM are on the same time scale as those on the s-PSM, indirectly disconfirming the formation of membrane extrusions as a rate limiting step.

An alternative interpretation of $N$ invokes a set of parallel activations with one ratelimiting step, e.g. displacement of the syb 2 fragment. As the displacement of the syb 2 fragment is a stochastic process, it can also be described with a gamma distribution [112, 152]. In the simplest way, $N=2$ could represent the average number of loosely bound SNARE-complexes, which need to displace the syb 2 fragment to fully zipper and initiate fusion in a concerted fashion. The assumption that the parallel displacement of the syb 2 fragment from two complexes is the overall rate limiting step would also explain why the docking times are independent of the vesicle size and the fusion site in all experiments.

Many groups tried to estimate the number of SNARE-complexes required to drive fusion, but the results are highly variable. While electrophysiological studies showed that five to eight SNAREs contribute to a fusion pore, dense core granules in pheochromocytoma12 cells required only three SNARE complexes for fusion [160, 161]. In vitro Förster 
Resonance Energy Transfer (FRET) experiments even propose a single SNARE complex for fusion between two vesicles. Additionally, recent studies from Hernandez et al. hypothesized that vesicles with a diameter of $100 \mathrm{~nm}$ require more SNAREs for fusion than smaller, $50 \mathrm{~nm}$ vesicles [128]. Despite the large deviations between the different studies, it seems well conceivable that multiple SNARE complexes are involved in fusing syb 2 vesicles.

The kinetic analysis of the single vesicle fusion events on s-PSMs revealed docking times of $16.7 \mathrm{~s}$ and $12.5 \mathrm{~s}$ for syb 2 vesicles with mean diameters of $90 \mathrm{~nm}$ and $240 \mathrm{~nm}$, respectively. The results are in good agreement with those obtained for $325 \mathrm{~nm}$ vesicles by the former co-worker Lando Schwenen [56]. The long docking times can be reasonably explained with the displacement of the syb 2 fragment from the stabilized $\Delta \mathrm{N}$-complex, which also takes several seconds and therefore resembles the rate limiting step in the docked state. These findings clearly prove SNARE specific fusion on PSMs. From the shape of the docking time distribution, it could be derived that docking to fusion involves a two step process. This is explained with a model including two SNARE-complexes per vesicle, which statistically displace their syb 2 fragment to initiate fusion in a concerted mechanism. 


\section{Conclusion}

The plasma membrane of eukaryotic cells is attached to the cytoskeleton, which significantly influences the lateral tension of the membrane. Lateral membrane tension was shown to be an important physical regulator of cellular processes such as cell motility and morphology as well as exo- and endocytosis. To mimic the plasma membrane and the underlying cytoskeleton in vitro, pore-spanning membranes (PSMs) were developed as an artificial model system. PSMs are composed of supported and partly freestanding membrane parts on a porous micro chip, which display lateral membrane tension. The lateral membrane tension and the planar geometry makes PSMs a versatile model system to study membrane processes by fluorescence and atomic force microscopy.

In this thesis, the lateral membrane tension of PSMs was modulated using well defined binary surface functionalizations on the pore rims of the porous substrates and subsequently analyzed by means of atomic force microscopy. Additionally, PSMs were utilized to analyze SNARE-mediated fusion on a single vesicle level to gain insights into the dynamics of this process on the different membrane parts. Therefore, fusion on supported and freestanding membranes was compared and kinetics were analyzed as a function of the vesicle size.

To modulate the lateral membrane tension of PSMs, binary 6-mercapto-1-hexanol (6$\mathrm{MH}$ ) and cholesterylpolyethylenoxythiol (CPEO3) self-assembled monolayers (SAMs) were established on the pore rims of gold coated porous substrates as a variable and versatile surface functionalization. It was verified that the composition of the binary SAM can be controlled by the ratio of the two thiols in the assembly solution. Contact angle and infrared reflection absorption spectroscopy allowed to estimate the amount of CPEO3 in the binary SAM. 1,2-diphytanoyl-sn-glycero-3-phosphocholine DPhPC PSMs were prepared on such 6-MH/CPEO3 SAMs containing different amounts of CPEO3, and their lateral membrane tension was measured by force indentation experiments. The lateral tension of the PSMs increased when larger mole fractions of CPEO3 were in the binary SAM due to an increasing adhesion energy of the 
membrane to the SAM. PSMs on SAMs containing no CPEO3 displayed a mean lateral tension of $1.4 \mathrm{mN} \mathrm{m}^{-1}$, while those on SAMs with $35 \mathrm{~mol} \%$ CPEO3 had a more than three times higher mean lateral tension of $4.5 \mathrm{mN} \mathrm{m}^{-1}$. The results demonstrate that the tension of the PSMs can be directly modified by the amount of CPEO3 in the SAM. Yet, the rather high tensions lead to spontaneous rupturing of the PSMs indicating a decreased stability. PSMs composed of physiological lipid mixtures were even more instable on SAMs containing CPEO3, which prevented further investigations of SNARE-mediated fusion as a function of membrane tension in PSMs. Therefore, SNARE-mediated fusion of vesicles with PSMs on pure 6-MH SAMs was further investigated by high speed spinning disc microscopy on a single vesicle level, as these membranes resembled sufficient stability and moderate membrane tensions. SNAREs, heterologously expressed in E. coli, were isolated, purified and functionally reconstituted into liposomes and PSMs. SNARE-specific activity was verified by bulk lipid mixing experiments as well as in the single vesicle assay. The mobility of the SNAREs in the supported membrane was analyzed by indirect FRAP experiments using a SNARE-transmembrane domain (SNARE-TMD) yielding a diffusion coefficient of $1 \mu^{2} \mathrm{~s}^{-1}$. Dynamic analysis of SNARE-docked vesicles revealed that vesicles on supported membranes are immobile, while those on freestanding membranes are mobile with a diffusion coefficient of $0.42 \mu \mathrm{m}^{2} \mathrm{~s}^{-1}$. Mobile SNARE-docked vesicles are unique in this system and were not observed in other artificial fusion assays based on planar membranes so far, indicating that interactions between the membrane support and the SNAREs lead to the observed immobilization of vesicles on supported membranes. Vesicles docked to the freestanding membrane had an eight times reduced mobility compared to the single SNARE-TMD indicating a close contact of the two membranes induced by the tight SNARE-complex. The time between docking and the onset of fusion, called docking time, of the vesicles on freestanding and supported membranes were on the time scale of seconds and showed no significant differences indicating a similar fusion mechanism on both membrane parts. This observations implies that fusion is unaffected by the higher mobility and lateral tension in the freestanding membrane. Kinetic analysis of the docking time distributions revealed a multiple step process, which can be interpreted with the assembly of two SNARE complexes or a kinetic intermediate. The influence of membrane curvature on the fusion kinetics was also analyzed, using vesicles with small $(90 \mathrm{~nm})$ and large $(240 \mathrm{~nm})$ diameters. The kinetics showed very similar docking time distributions suggesting that fusion is not significantly influenced by the different curvatures of the vesicles. Analysis of the time 
resolved merging of the vesicle structure with the supported membrane upon fusion revealed that nearly half of the vesicles fully merge with the PSMs, while the other half showed semi-stable post fusion structures, which could be a result of a hindered content release under the membrane.

PSMs were successfully used as an artificial model membrane system to modulate lateral membrane tension and to gather new insights into the kinetics of SNAREmediated fusion on supported and freestanding membranes. In combination with high speed spinning disc microscopy, this assay allowed to analyze dynamic aspects of the fusion process in more detail. 



\section{Appendix}

\subsection{Amino acid sequences of SNAREs}

\subsubsection{Amino acid sequence of synaptobrevin 2}

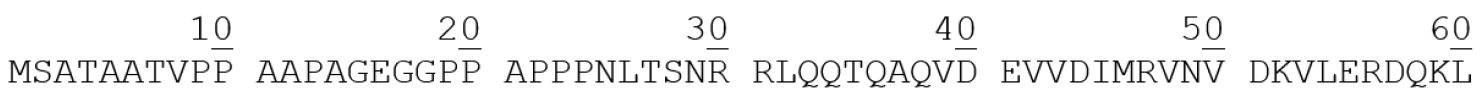

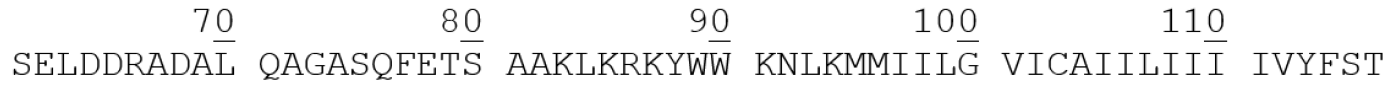

Figure 9.1. Amino acid sequence of synaptobrevin 2 (aa 1-116).

\subsubsection{Amino acid sequence of syntaxin $1 \mathrm{~A}$}

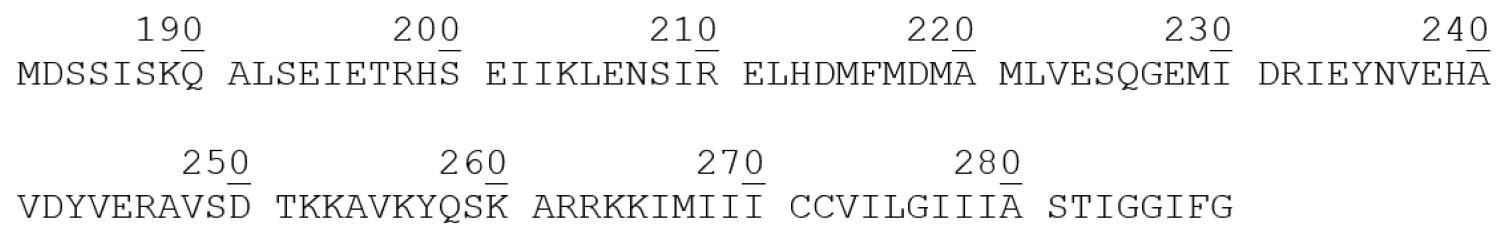

Figure 9.2. Amino acid sequence of syntaxin 1A (aa 183-288).

\subsubsection{Amino acid sequence of syntaxin 1A-transmembrane domain}

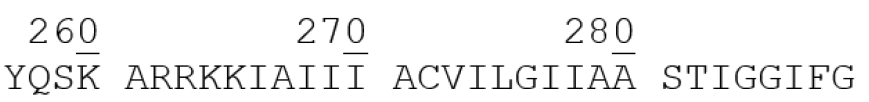

Figure 9.3. Amino acid sequence of syntaxin 1A transmembrane domain (aa 257-288). 


\subsubsection{Amino acid sequence of SNAP 25a}

\begin{tabular}{|c|c|c|c|c|c|}
\hline $1 \underline{0}$ & $2 \underline{0}$ & $3 \underline{0}$ & $4 \underline{0}$ & $5 \underline{0}$ & $6 \underline{0}$ \\
\hline MAEDADMRNE & LEEMQRRADQ & LADESLESTR & RMLQLVEESK & DAGIRTLVML & DEQGEQLDRV \\
\hline $7 \underline{0}$ & $8 \underline{0}$ & $9 \underline{0}$ & $10 \underline{0}$ & $11 \underline{0}$ & $12 \underline{0}$ \\
\hline EEGMNH INQD & MKEAEKNLKD & 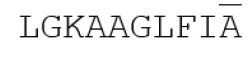 & PANKLKSSDA & YKKAWGNNQD̄ & GVVASQPARV \\
\hline $13 \underline{0}$ & $14 \underline{0}$ & $15 \underline{0}$ & $16 \underline{0}$ & $17 \underline{0}$ & $18 \underline{0}$ \\
\hline VDEREQMAIS & GGFIRRVTND & ARENEMDENL & EQVSGIIGNL & RHMALDMGNE & IDTQNRQIDR \\
\hline $19 \underline{0}$ & $20 \underline{0}$ & & & & \\
\hline MEKA & RIDEANQRAT & KMLGSG & & & \\
\hline
\end{tabular}

Figure 9.4. Amino acid sequence of SNAP 25a (aa 1-206).

\subsection{List of symbols and abbreviations}

$\begin{array}{ll}6 \text {-MH } & \text { 6-Mercapto-1-hexanol } \\ \text { aa } & \text { amino acid } \\ \text { AFM } & \text { atomic force microscope } \\ c & \text { concentration } \\ \text { CHAPS } & 3 \text {-[(3-cholamidopropyl)-dimethylammonio }]- \\ & \text { propanesulfonate } \\ \text { Chol } & \text { cholesterol } \\ \text { CLSM } & \text { confocal laser scanning microscope } \\ \text { CPEO3 } & \text { cholesterolpolyethylenoxythiole } \\ D & \text { diffusion coefficient } \\ \text { DOPC } & 1,2 \text {-dioleoyl-sn-glycero-3-phosphocholine } \\ \text { DPhPC } & \text { Diphytanoyl phosphatidylcholine } \\ \text { DPPC } & 1,2 \text {-dipalmitoyl-sn-glycero-3-phosphocholine } \\ \text { DPPE } & 1,2 \text {-dipalmitoyl-sn-glycero-3-phosphoethanolamine } \\ \text { DTT } & \text { dithiothreitol } \\ E . C \text { Coli } & \text { Escherichia coli } \\ F & \text { force } \\ \text { FCS } & \text { fluorescence correlation spectroscopy } \\ \text { FD } & \text { force distance } \\ \text { f-PSM } & \text { freestanding pore spanning membrane } \\ & \end{array}$


FRAP

FRET

GUV

$\mathrm{h}$

h

HEPES

His

ITO

k

$\mathrm{KCl}$

$\mathrm{kDa}$

LUV

$\lambda$

M

$\mathrm{M}$

$\min$

ms

NSF

pdf

PM-IRRAS

POPE

POPS

PSM

ROI

RT

$s$

SDCM

SDS-PAGE

SEM

SNAP

SNARE

s-PSM

SSM

SUV fluorescence recovery after photobleaching

Förster-Resonance-Energy-Transfer

giant unilamellar vesicle

hour

height

2-[4-(2-hydroxyethyl)piperazin-1-yl]ethanesulfonic acid

histidin

indium tin oxide

spring constant

sodiumchloride

kilo Dalton

large unilamellar vesicle

wave length

molar mass

molar

minute

miliseconds

NEM-sensitive factor

probability density function

polarization modulation infrared reflection absorption spectroscopy

1-Palmitoyl-2-oleoyl-sn-glycero-3-phosphoethanolamine

1-Palmitoyl-2-oleoyl-sn-glycero-3-phospho-l-serine

pore-spanning membrane

region of interest

room temperature

second

spinning disc confocal microscopy

sodium dodecylsulfat polyacrylamide gel electrophoresis

scanning electron microscope

soluble NSF-attachment protein

SNAP receptor

supported pore spanning membrane

solid supported membrane

small unilamellar vesicle 


$\begin{array}{ll}\text { syb } 2 & \text { synaptobrevin } 2 \\ \text { syx } 1 \mathrm{~A} & \text { syntaxin } 1 \mathrm{~A} \\ t & \text { time } \\ \text { TIRF } & \text { total internal reflection fluorescence } \\ \text { TR } & \text { TexasRed-DPPE } \\ \mathrm{UV} & \text { ultraviolett } \\ \text { vis } & \text { visible }\end{array}$

\subsection{List of chemicals and consumables}

\begin{tabular}{|c|c|}
\hline Argon & Linde (München) \\
\hline Chloroform & VWR International (Darmstadt) \\
\hline Ethanol & Fluka (Neu-Ulm) \\
\hline Mucasol@ & Merck (Darmstadt) \\
\hline Parafilm & Pechiney Plastik Packaging (Chicago, USA) \\
\hline Petridishes & VWR International GmbH, (Nürnberg) \\
\hline $\mathrm{DOPC}$ & Avanti Polar Lipids (Alabaster, USA) \\
\hline POPE & Avanti Polar Lipids (Alabaster, USA) \\
\hline POPS & Avanti Polar Lipids (Alabaster, USA) \\
\hline Cholesterol & Sigma-Aldrich (Taufkirchen) \\
\hline TexasRed-DHPE & Sigma-Aldrich (Taufkirchen) \\
\hline Atto488-DPPE & Atto-tec (Siegen) \\
\hline HEPES & Carl Roth GmbH (Karlsruhe) \\
\hline $\mathrm{KCl}$ & Carl Roth GmbH (Karlsruhe) \\
\hline DTT & Sigma-Aldrich (Taufkirchen) \\
\hline Sucrose & Carl Roth (Karlsruhe) \\
\hline ITO-Slides & Präzisions Glas \& Optik GmbH (Iserlohn) \\
\hline Copperband & Präzisions Glas \& Optik GmbH (Iserlohn) \\
\hline Polycarbonatemembrane (50 nm + & Avestin (Ontario, Canada) \\
\hline $400 \mathrm{~nm})$ & \\
\hline Eppendorf-Cups & Eppendorf (Hamburg) \\
\hline Eppendorfpipettes & Eppendorf (Hamburg) \\
\hline Porous substrates $(1.2 \mu \mathrm{m})$ & fluXXion (Eindhofen, The Netherlands) \\
\hline Porous substrates $(5.0 \mu \mathrm{m})$ & Aquamarijn (Zutphen, The Netherlands) \\
\hline Titan & Elektronen Optik Service GmbH (Dortmund) \\
\hline
\end{tabular}


Gold, $99.99 \%$

OG-syx 1A-TMD
Allgemeine Gold- und Silberscheideanstalt (Pforzheim) Provided by Prof. Dr. Ulf Diederichsen

\subsection{List of devices and software}

atomic force microscopy

MFP 3D Asylum Research (CA, USA)

\section{fluorescence microscopy}

$\begin{array}{ll}\text { Olympus BX 51 } & \text { Olympus (Tokio, Japan) } \\ \text { LumPlan FLN 40x/0.80 W } & \text { Olympus (Tokio, Japan) } \\ \text { LumPlan FLN 100x/1.00 W } & \text { Olympus (Tokio, Japan) } \\ \text { XCite Series 120 Q } & \text { Olympus (Tokio, Japan) }\end{array}$

confocal laser scanning microscopy (CLSM)

Olympus FV $1200 \quad$ Olympus (Tokio, Japan)

BX $61 \quad$ Olympus (Tokio, Japan)

spinning disc confocal scanning microscopy (SDCM)

stand: custom, based on Olympus (Tokio, Japan)

ix73

spinning disc: Yokogawa Rota Yokogawa GmbH \& Co. KG (Wehr, Ger)

CSU-X

AOTF: $\quad$ TF525-250-6-3- Gooch \& Housego PLC (Ilminster, UK)

GH18A

laser: iBeam Smart 488-S Toptica Photonics AG (Gräflingen, Ger)

laser: iBeam Smart 561-S Toptica Photonics AG (Gräflingen, Ger)

laser: Cobolt Jive ${ }^{\mathrm{TM}} \quad$ Cobolt AB, Solna (Sweden)

camera: iXON 897 Ultra Andor Technology Ltd. (Belfast, UK)

filter: LP590 ET

AHF Analysentechnik AG (Tübingen, Ger)

filter: BP450/50 ET

AHF Analysentechnik AG (Tübingen, Ger)

filter: BP650/60 ET

AHF Analysentechnik AG (Tübingen, Ger)

filter: ZET405/488/561/640 AHF Analysentechnik AG (Tübingen, Ger) 
objective: LUMFLN 60XW Olympus (Hamburg, Ger)

60x NA 1.1

contact angle measurements

stand: custom

camera: Nikon D600 Nikon (Minato, Tokio)

objective: AF-S Nikkor Nikon (Minato, Tokio)

$18 / 300 \mathrm{~mm}$

lens: custom made $\quad$ Scientific Precision Instruments GmbH (Oppenheim, Ger)

polarization modulation infrared reflection absorption spectroscopy

FT-IR: Vertex 70

Bruker (MA, USA)

module: PMA50

Bruker (MA, USA)

sample holder: PM-IRRAS Bruker (MA, USA)

A196/B

photoelastic modulator Hinds Instruments (OR, USA)

PEM90

surface coating

Zepto plasma cleaner Diener Electronic (Ebbhausen, Ger)

Coating System MED020 Bal-Tec, Leica (Wetzlar, Ger)

Sputtercoater 108 auto Cressington Scientific Instruments (Watford, UK)

miscelaneous

NanoDrop2000c

Thermo Scientific (Wilmington, USA)

pH-meter: Calimatic 766

Knick (Berlin, Ger)

liposofast extruder

Avestin (Ottawa, USA)

frequency generator: Agilent

Agilent Technologies (Santa Clara, USA)

$32220 \mathrm{~A}$

Äkta purification system

GE Healthcare (Little Chalfont, UK)

MilliQ Gradient A10

Millipore (Eschborn, Ger)

UV-vis: JASCO FP 6500

JASCO (Easton, USA) 


\subsection{Software}

ImageJ 1.45

Origin $8.5 \mathrm{G}$

Matlab2014a

IQ 2.9

OPUS 6.5 http://rsbweb.nih.gov/ij

OriginLab Cooperation (Northampton, USA)

MathWorks (Natick, USA)

Andor Technology Ltd. (Belfast, UK)

Bruker (MA, USA)

\subsection{Composition of expression media}

Table 9.12. Composition of the expression media.

\begin{tabular}{lll}
\hline Terrific broth (TB)-medium & $\begin{array}{l}\text { Tryptone } \\
\text { Yeast extract } \\
\text { Glycerin }\end{array}$ & $\begin{array}{c}30 \mathrm{~g} \\
60 \mathrm{~g} \\
10 \mathrm{~g}\end{array}$ \\
& $\mathrm{~K}_{2} \mathrm{HPO}_{4} \mathrm{xH}_{2} \mathrm{O}$ & $41 \mathrm{~g}$ \\
Terrific Broth (TB)-salt & $\mathrm{KH}_{2} \mathrm{PO}_{4}$ & $5.8 \mathrm{~g}$ \\
$(250 \mathrm{ml})$ & $\begin{array}{l}\text { Tryptone } \\
\text { Lysogeny Broth (LB)-medium }\end{array}$ & $5 \mathrm{~g}$ \\
$(500 \mathrm{ml})$ & $\begin{array}{l}\text { Yeast extract } \\
\text { Sodium chloride }\end{array}$ & $5 \mathrm{~g}$
\end{tabular}




\subsection{Composition of SDS-PAGE buffer}

Table 9.13. Buffer composition used for SDS-PAGE.

\begin{tabular}{|c|c|c|}
\hline & Chemicals & Concentration \\
\hline $\begin{array}{l}\text { Cathode buffer } \\
(10 \mathrm{x})\end{array}$ & $\begin{array}{l}\text { Tris } \\
\text { Tricin } \\
\text { SDS }\end{array}$ & $\begin{array}{l}1 \mathrm{M} \\
1 \mathrm{M} \\
1 \%(\mathrm{w} / \mathrm{v})\end{array}$ \\
\hline $\begin{array}{l}\text { Anode buffer } \\
(10 \mathrm{x}, \mathrm{pH}=8.9)\end{array}$ & Tris/HCl & $2 \mathrm{M}$ \\
\hline Gel buffer & $\begin{array}{l}\text { Tris } / \mathrm{HCl} \\
\text { SDS }\end{array}$ & $\begin{array}{l}3 \mathrm{M} \\
0.3 \%(\mathrm{w} / \mathrm{v})\end{array}$ \\
\hline Probe buffer & $\begin{array}{l}\text { Tris/HCl } \\
\text { SDS } \\
\text { Glycerin } \\
\text { DTT } \\
\text { Bromphenol blue }\end{array}$ & $\begin{array}{l}175 \mathrm{mM} \\
5 \%(\mathrm{w} / \mathrm{v}) \\
15 \%(\mathrm{w} / \mathrm{v}) \\
0.3 \mathrm{mM} \\
0.02 \%(\mathrm{w} / \mathrm{v})\end{array}$ \\
\hline Acrylamide solution & $\begin{array}{l}\text { Acrylamide/ } \\
\text { Bisacrylamide (29:1) }\end{array}$ & $30 \%$ \\
\hline
\end{tabular}




\subsection{Composition of SDS-PAGE gels}

Table 9.14. Composition of a $10 \%$ acrylamide gel.

\begin{tabular}{lll}
\hline & Stacking gel & Resolving gel \\
\hline Acrylamide & $200 \mu \mathrm{l}$ & $1.6 \mathrm{ml}$ \\
Gel buffer & $375 \mu \mathrm{l}$ & $1.675 \mathrm{ml}$ \\
$\mathrm{H}_{2} \mathrm{O}$ & $925 \mu \mathrm{l}$ & $570 \mu \mathrm{l}$ \\
Glycerin $(50 \%)$ & - & $1.06 \mathrm{ml}$ \\
APS $(10 \%)$ & $10 \mu \mathrm{l}$ & $25 \mu \mathrm{l}$ \\
TEMED & $2 \mu \mathrm{l}$ & $25 \mu \mathrm{l}$ \\
\hline
\end{tabular}




\subsection{Compositions of isolation buffers.}

Table 9.15. Buffer composition used for the isolation of SNAP 25a.

\begin{tabular}{|c|c|c|}
\hline & Chemicals & Concentration \\
\hline Extraction buffer & $\begin{array}{l}\text { Tris/HCl } \\
\mathrm{NaCl} \\
\text { Imidazol } \\
\text { Sodium cholate }\end{array}$ & $\begin{array}{l}20 \mathrm{mM} \mathrm{pH} 7.4 \\
500 \mathrm{mM} \\
20 \mathrm{mM} \\
10 \%\end{array}$ \\
\hline Washing buffer I & $\begin{array}{l}\text { Tris } / \mathrm{HCl} \\
\mathrm{NaCl} \\
\text { Imidazol }\end{array}$ & $\begin{array}{l}20 \mathrm{mM} \mathrm{pH} 7.4 \\
500 \mathrm{mM} \\
20 \mathrm{mM}\end{array}$ \\
\hline Elution buffer & $\begin{array}{l}\text { Tris/HCl } \\
\mathrm{NaCl} \\
\text { Imidazol } \\
\text { DTT }\end{array}$ & $\begin{array}{l}20 \mathrm{mM} \mathrm{pH} 7.4 \\
500 \mathrm{mM} \\
400 \mathrm{mM} \\
1 \mathrm{mM}\end{array}$ \\
\hline Dialysis buffer & $\begin{array}{l}\mathrm{HEPES} / \mathrm{NaOH} \\
\mathrm{NaCl} \\
\text { EDTA } \\
\text { DTT }\end{array}$ & $\begin{array}{l}20 \mathrm{mM} \mathrm{pH} 7.4 \\
50 \mathrm{mM} \\
1 \mathrm{mM} \\
1 \mathrm{mM}\end{array}$ \\
\hline Äkta buffer A & $\begin{array}{l}\mathrm{HEPES} / \mathrm{NaOH} \\
\text { EDTA } \\
\text { DTT }\end{array}$ & $\begin{array}{l}20 \mathrm{mM} \mathrm{pH} 7.4 \\
1 \mathrm{mM} \\
1 \mathrm{mM}\end{array}$ \\
\hline Äkta buffer B & $\begin{array}{l}\mathrm{HEPES} / \mathrm{NaOH} \\
\mathrm{NaCl} \\
\text { EDTA } \\
\text { DTT }\end{array}$ & $\begin{array}{l}20 \mathrm{mM} \mathrm{pH} 7.4 \\
1 \mathrm{M} \\
1 \mathrm{mM} \\
1 \mathrm{mM}\end{array}$ \\
\hline
\end{tabular}


Table 9.16. Buffer composition used for the isolation of synaptobrevin 2 and synaptobrevin 2 C79 (residues 49-96).

\begin{tabular}{|c|c|c|}
\hline & Chemicals & Concentration \\
\hline Extraction buffer & $\begin{array}{l}\text { Tris/HCl } \\
\mathrm{NaCl} \\
\text { Imidazol } \\
\text { Sodium cholate }\end{array}$ & $\begin{array}{l}20 \mathrm{mM} \mathrm{pH} 7.4 \\
500 \mathrm{mM} \\
20 \mathrm{mM} \\
10 \%\end{array}$ \\
\hline Washing buffer I & $\begin{array}{l}\text { Tris } / \mathrm{HCl} \\
\mathrm{NaCl} \\
\text { Imidazol } \\
\text { Sodium cholate }\end{array}$ & $\begin{array}{l}20 \mathrm{mM} \mathrm{pH} 7.4 \\
500 \mathrm{mM} \\
20 \mathrm{mM} \\
1 \%\end{array}$ \\
\hline Washing buffer II & $\begin{array}{l}\text { Tris/HCl } \\
\mathrm{NaCl} \\
\text { Imidazol } \\
\text { CHAPS }\end{array}$ & $\begin{array}{l}20 \mathrm{mM} \mathrm{pH} 7.4 \\
500 \mathrm{mM} \\
20 \mathrm{mM} \\
1 \%\end{array}$ \\
\hline Elution buffer & $\begin{array}{l}\text { Tris/HCl } \\
\mathrm{NaCl} \\
\text { Imidazol } \\
\text { CHAPS }\end{array}$ & $\begin{array}{l}20 \mathrm{mM} \mathrm{pH} 7.4 \\
500 \mathrm{mM} \\
400 \mathrm{mM} \\
1 \%\end{array}$ \\
\hline Dialysis buffer & $\begin{array}{l}\mathrm{HEPES} / \mathrm{NaOH} \\
\mathrm{NaCl} \\
\text { EDTA } \\
\text { DTT } \\
\text { CHAPS }\end{array}$ & $\begin{array}{l}20 \mathrm{mM} \mathrm{pH} 7.4 \\
150 \mathrm{mM} \\
1 \mathrm{mM} \\
1 \mathrm{mM} \\
1 \%\end{array}$ \\
\hline Äkta buffer A & $\begin{array}{l}\text { HEPES/NaOH } \\
\text { EDTA } \\
\text { DTT } \\
\text { CHAPS }\end{array}$ & $\begin{array}{l}20 \mathrm{mM} \mathrm{pH} 7.4 \\
1 \mathrm{mM} \\
1 \mathrm{mM} \\
1 \%\end{array}$ \\
\hline Äkta buffer B & $\begin{array}{l}\mathrm{HEPES} / \mathrm{NaOH} \\
\mathrm{NaCl} \\
\text { EDTA } \\
\text { DTT } \\
\text { CHAPS }\end{array}$ & $\begin{array}{l}20 \mathrm{mM} \mathrm{pH} 7.4 \\
1000 \mathrm{mM} \\
1 \mathrm{mM} \\
1 \mathrm{mM} \\
1 \%\end{array}$ \\
\hline
\end{tabular}


Table 9.17. Buffer composition used for the isolation of syntaxin $1 \mathrm{~A}$.

\begin{tabular}{|c|c|c|}
\hline & Chemicals & Concentration \\
\hline \multirow[t]{4}{*}{ Extraction buffer } & Tris/HCl & $20 \mathrm{mM} \mathrm{pH} 7.4$ \\
\hline & $\mathrm{NaCl}$ & $500 \mathrm{mM}$ \\
\hline & Imidazol & $20 \mathrm{mM}$ \\
\hline & Sodium cholate & $10 \%$ \\
\hline \multirow[t]{5}{*}{ Washing buffer } & Tris/HCl & $20 \mathrm{mM} \mathrm{pH} 7.4$ \\
\hline & $\mathrm{NaCl}$ & $500 \mathrm{mM}$ \\
\hline & Imidazol & $20 \mathrm{mM}$ \\
\hline & Sodium cholate & $3 \%$ \\
\hline & Urea & $6 \mathrm{M}$ \\
\hline \multirow[t]{5}{*}{ Elution buffer } & Tris/HCl & $20 \mathrm{mM} \mathrm{pH} 7.4$ \\
\hline & $\mathrm{NaCl}$ & $500 \mathrm{mM}$ \\
\hline & Imidazol & $400 \mathrm{mM}$ \\
\hline & Sodium cholate & $3 \%$ \\
\hline & Urea & $6 \mathrm{M}$ \\
\hline \multirow[t]{6}{*}{ Dialysis buffer I } & Tris/HCl & $20 \mathrm{mM} \mathrm{pH} 7.4$ \\
\hline & $\mathrm{NaCl}$ & $500 \mathrm{mM}$ \\
\hline & EDTA & $1 \mathrm{mM}$ \\
\hline & DTT & $1 \mathrm{mM}$ \\
\hline & Sodium cholate & $3 \%$ \\
\hline & Urea & $2 \mathrm{M}$ \\
\hline \multirow[t]{5}{*}{ Dialysis buffer II } & HEPES/NaOH & $20 \mathrm{mM} \mathrm{pH} 7.4$ \\
\hline & $\mathrm{NaCl}$ & $150 \mathrm{mM}$ \\
\hline & EDTA & $1 \mathrm{mM}$ \\
\hline & DTT & $1 \mathrm{mM}$ \\
\hline & CHAPS & $1 \%$ \\
\hline \multirow[t]{5}{*}{ Äkta buffer A } & HEPES/NaOH & $20 \mathrm{mM} \mathrm{pH} 7.4$ \\
\hline & $\mathrm{NaCl}$ & $250 \mathrm{mM}$ \\
\hline & EDTA & $1 \mathrm{mM}$ \\
\hline & DTT & $1 \mathrm{mM}$ \\
\hline & CHAPS & $1 \%$ \\
\hline \multirow[t]{5}{*}{ Äkta buffer B } & HEPES/NaOH & $20 \mathrm{mM} \mathrm{pH} 7.4$ \\
\hline & $\mathrm{NaCl}$ & $1000 \mathrm{mM}$ \\
\hline & EDTA & $1 \mathrm{mM}$ \\
\hline & DTT & $1 \mathrm{mM}$ \\
\hline & CHAPS & $1 \%$ \\
\hline
\end{tabular}




\section{Bibliography}

[1] M. P. Sheetz, J. Dai, Modulation of membrane dynamics and cell motility by membrane tension, Trends Cell Biol. 1996, 6(3), 85-89.

[2] A. Diz-Munoz, D. A. Fletcher, O. D. Weiner, Use the force: membrane tension as an organizer of cell shape and motility, Trends Cell Biol. 2013, 23(2), 47-53.

[3] N. C. Gauthier, T. A. Masters, M. P. Sheetz, Mechanical feedback between membrane tension and dynamics., Trends Cell Biol. 2012, 22(10), 527-535.

[4] J. R. Monck, G. Alvarez de Toledo, J. M. Fernandez, Tension in secretory granule membranes causes extensive membrane transfer through the exocytotic fusion pore., Proc. Natl. Acad. Sci. U.S.A. 1990, 87(20), 7804-7808.

[5] M. Kocun, T. D. Lazzara, C. Steinem, A. Janshoff, Preparation of solvent-free, pore-spanning lipid bilayers: modeling the low tension of plasma membranes, Langmuir 2011, 27(12), 7672-7680.

[6] A. D. Lieber, S. Yehudai-Resheff, E. L. Barnhart, E. A. Barnhart, K. Keren, Membrane tension in rapidly moving cells is determined by cytoskeletal forces, Curr. Biol. 2013, 23(15), 1409-1417.

[7] C. Morris, U. Homann, Cell surface area regulation and membrane tension, $J$. Membr. Biol. 2001, 179(2), 79-102.

[8] Y. Schweitzer, A. D. Lieber, K. Keren, M. M. Kozlov, Theoretical analysis of membrane tension in moving cells, Biophys. J. 2014, 106(1), 84-92.

[9] A. Pietuch, B. R. Brückner, A. Janshoff, Membrane tension homeostasis of epithelial cells through surface area regulation in response to osmotic stress., Biochim. Biophys. Acta 2013, $1833(3), 712-722$.

[10] M. P. Sheetz, Cell control by membrane-cytoskeleton adhesion, Nat. Rev. Mol. Cell Biol. 2001, 2(5), 392-396. 
[11] N. C. Gauthier, M. A. Fardin, P. Roca-Cusachs, M. P. Sheetz, Temporary increase in plasma membrane tension coordinates the activation of exocytosis and contraction during cell spreading., Proc. Natl. Acad. Sci. U.S.A. 2011, $108(35), 14467-14472$.

[12] M. Staykova, D. P. Holmes, C. Read, H. A. Stone, Mechanics of surface area regulation in cells examined with confined lipid membranes, Proc. Natl. Acad. Sci. U.S.A. 2011, 108(22), 9084-9088.

[13] J. C. Shillcock, R. Lipowsky, Tension-induced fusion of bilayer membranes and vesicles, Nat. Mater. 2005, 4(3), 225-228.

[14] H. J. Risselada, C. Kutzner, H. Grubmüller, Caught in the act: visualization of SNARE-mediated fusion events in molecular detail, ChemBioChem 2011, 12(7), 1049-1055.

[15] W. Antonin, D. Fasshauer, S. Becker, R. Jahn, T. R. Schneider, Crystal structure of the endosomal SNARE complex reveals common structural principles of all SNAREs, Nat. Struct. Biol. 2002, 9(2), 107-111.

[16] R. Jahn, T. C. Südhof, Membrane fusion and exocytosis, Annu. Rev. Biochem. 1999, 68(1), 863-911.

[17] M. Wölfel, R. Schneggenburger, Presynaptic capacitance measurements and $\mathrm{Ca}^{2+}$ uncaging reveal submillisecond exocytosis kinetics and characterize the $\mathrm{Ca}^{2+}$ sensitivity of vesicle pool depletion at a fast CNS synapse, J. Neuro Sci. 2003, 23(18), 7059-7068.

[18] R. Jahn, R. H. Scheller, SNAREs - engines for membrane fusion, Nature Reviews Molecular Cell Biology 2006, 7(9), 631-643.

[19] R. Jahn, D. Fasshauer, Molecular machines governing exocytosis of synaptic vesicles, Nature 2012, 490(7419), 201-207.

[20] V. Malhotra, L. Orci, B. Glick, M. Block, J. Rothman, Role of an Nethylmaleimide-sensitive transport component in promoting fusion of transport vesicles with cisternae of the Golgi stack, Cell 1988, 54(2), 221-227.

[21] M. R. Block, B. S. Glick, C. A. Wilcox, F. T. Wieland, J. E. Rothman, Purification of an N-ethylmaleimide-sensitive protein catalyzing vesicular transport., Proc. Natl. Acad. Sci. U. S. A. 1988, 85(21), 7852-7856. 
[22] D. Clary, I. Griff, J. Rothman, SNAPs, a family of NSF attachment proteins involved in intracellular membrane fusion in animals and yeast., Cell 1990, 61(4), 709-721.

[23] T. Söllner, S. W. Whiteheart, M. Brunner, H. Erdjument-Bromage, S. Geromanos, P. Tempst, J. E. Rothman, SNAP receptors implicated in vesicle targeting and fusion, Nature 1993, 362(6418), 318-324.

[24] T. Weber, B. V. Zemelman, J. A. McNew, B. Westermann, M. Gmachl, F. Parlati, T. H. Söllner, J. E. Rothman, SNAREpins: minimal machinery for membrane fusion, Cell 1998, 92(6), 759-772.

[25] D. Fasshauer, R. B. Sutton, A. T. Brunger, R. Jahn, Conserved structural features of the synaptic fusion complex: SNARE proteins reclassified as Q- and R-SNAREs, Proc. Natl. Acad. Sci. USA 1998, 95(26), 15781-15786.

[26] P. Burkhardt, The origin and evolution of synaptic proteins - choanoflagellates lead the way, J. Exp. Biol. 2015, 218(4), 506-514.

[27] R. B. Sutton, D. Fasshauer, R. Jahn, A. T. Brunger, Crystal structure of a SNARE complex involved in synaptic exocytosis at 2.4 A resolution, Nature 1998, 395 (6700), 347-353.

[28] A. V. Pobbati, A. Stein, D. Fasshauer, N- to C-terminal SNARE complex assembly promotes rapid membrane fusion, Science 2006, 313(5787), 673-676.

[29] L. Yang, H. W. Huang, A rhombohedral phase of lipid containing a membrane fusion intermediate structure, Biophys. J. 2003, 84(3), 1808-1817.

[30] S. Aeffner, T. Reusch, B. Weinhausen, T. Salditt, Energetics of stalk intermediates in membrane fusion are controlled by lipid composition, Proc. Natl. Acad. Sci. U. S. A. 2012, $109(25)$, E1609-E1618.

[31] J. Nikolaus, M. Stöckl, D. Langosch, R. Volkmer, A. Herrmann, Direct visualization of large and protein-free hemifusion diaphragms, Biophys. J. 2010, 98(7), 1192-1199.

[32] J. M. Hernandez, A. Stein, E. Behrmann, D. Riedel, A. Cypionka, Z. Farsi, P. J. Walla, S. Raunser, R. Jahn, Membrane fusion intermediates via directional and full assembly of the SNARE complex, Science 2012, 336(6088), 1581-1584. 
[33] J. Diao, P. Grob, D. J. Cipriano, M. Kyoung, Y. Zhang, S. Shah, A. Nguyen, M. Padolina, A. Srivastava, M. Vrljic, et al., Synaptic proteins promote calciumtriggered fast transition from point contact to full fusion, eLife 2012, 1:e00109.

[34] L. Chernomordik, M. Kozlov, G. Melikyan, I. Abidor, V. Markin, Y. Chizmadzhev, The shape of lipid molecules and monolayer membrane fusion, $B B A$ Biomembranes 1985, 812(3), 643-655.

[35] G. Basanez, F. M. Goni, A. Alonso, Effect of single chain lipids on phospholipase C-promoted vesicle fusion. a test for the stalk hypothesis of membrane fusion, Biochemistry 1998, 37(11), 3901-3908.

[36] L. V. Chernomordik, M. M. Kozlov, Mechanics of membrane fusion, Nat. Struct. Mol. Biol. 2008, 15(7), 675-683.

[37] M. Schick, Membrane fusion: the emergence of a new paradigm, J. Stat. Phys. 2010, 142(6), 1317-1323.

[38] Y. A. Chen, R. H. Scheller, SNARE-mediated membrane fusion, Nat. Rev. Mol. Cell Biol. 2001, 2(2), 98-106.

[39] A. T. Brunger, D. J. Cipriano, J. Diao, Towards reconstitution of membrane fusion mediated by SNAREs and other synaptic proteins, Crit. Rev. Biochem. Mol. Biol. 2015, 50(3), 231-241.

[40] D. K. Struck, D. Hoekstra, R. E. Pagano, Use of resonance energy transfer to monitor membrane fusion., Biochemistry 1981, 20(14), 4093-4099.

[41] L. Schwenen, Dissertation, Georg August University Göttingen, 2015.

[42] Y.-H. M. Chan, B. van Lengerich, S. G. Boxer, Effects of linker sequences on vesicle fusion mediated by lipid-anchored DNA oligonucleotides, Proc. Natl. Acad. Sci. USA 2009, 106(4), 979-984.

[43] J. Bai, R. E. Pagano, Measurement of spontaneous transfer and transbilayer movement of BODIPY-labeled lipids in lipid vesicles, Biochemistry 1997, 36(29), 8840-8848.

[44] S. Ohki, T. D. Flanagan, D. Hoekstra, Probe Transfer with and without Membrane Fusion in a Fluorescence Fusion Assay, Biochemistry 1998, 37(20), 74967503 . 
[45] T.-Y. Yoon, B. Okumus, F. Zhang, Y.-K. Shin, T. Ha, Multiple intermediates in SNARE-induced membrane fusion, Proc. Natl. Acad. Sci. USA 2006, 103(52), 19731-19736.

[46] M. Kyoung, A. Srivastava, Y. Zhang, J. Diao, M. Vrljic, P. Grob, E. Nogales, S. Chu, A. T. Brunger, In vitro system capable of differentiating fast $\mathrm{Ca}^{2+}$-triggered content mixing from lipid exchange for mechanistic studies of neurotransmitter release, Proc. Natl. Acad. Sci. USA 2011, 108(29), E304-E313.

[47] Y. Lai, J. Diao, D. J. Cipriano, Y. Zhang, R. A. Pfuetzner, M. S. Padolina, A. T. Brunger, Complexin inhibits spontaneous release and synchronizes $\mathrm{Ca}^{2+}$ triggered synaptic vesicle fusion by distinct mechanisms, eLife 2014, 3:e03756.

[48] M. E. Bowen, K. Weninger, A. T. Brunger, S. Chu, Single molecule observation of liposome-bilayer fusion thermally induced by soluble N-ethyl maleimide sensitivefactor attachment protein receptors (SNAREs), Biophys. J. 2004, 87(5), 35693584 .

[49] M. Fix, T. J. Melia, J. K. Jaiswal, J. Z. Rappoport, D. You, T. H. Sollner, J. E. Rothman, S. M. Simon, Imaging single membrane fusion events mediated by SNARE proteins, Proc. Natl. Acad. Sci. USA 2004, 101(19), 7311-7316.

[50] T. Liu, W. C. Tucker, A. Bhalla, E. R. Chapman, J. C. Weisshaar, SNARE-driven, 25-millisecond vesicle fusion in vitro, Biophys. J. 2005, 89(4), 2458-2472.

[51] T. Wang, E. A. Smith, E. R. Chapman, J. C. Weisshaar, Lipid mixing and content release in single-vesicle, SNARE-driven fusion assay with 1-5 ms resolution, Biophys. J. 2009, $96(10), 4122-4131$.

[52] E. Karatekin, J. E. Rothman, Fusion of single proteoliposomes with planar, cushioned bilayers in microfluidic flow cells, Nature Protocols 2012, 7(5), 903920.

[53] M. K. Domanska, V. Kiessling, A. Stein, D. Fasshauer, L. K. Tamm, Single vesicle millisecond fusion kinetics reveals number of SNARE complexes optimal for fast SNARE-mediated membrane fusion, J. Biol. Chem. 2009, 284(46), 32158-32166. 
[54] A. J. Kreutzberger, V. Kiessling, L. K. Tamm, High cholesterol obviates a prolonged hemifusion intermediate in fast SNARE-mediated membrane fusion, Biophys. J. 2015, $109(2)$, 319-329.

[55] I. Höfer, C. Steinem, A membrane fusion assay based on pore-spanning lipid bilayers, Soft Matter 2011, 7(5), 1644.

[56] L. L. G. Schwenen, R. Hubrich, D. Milovanovic, B. Geil, J. Yang, A. Kros, R. Jahn, C. Steinem, Resolving single membrane fusion events on planar porespanning membranes, Sci. Rep. 2015, 5, 1-15.

[57] D. Fasshauer, W. Antonin, V. Subramaniam, R. Jahn, SNARE assembly and disassembly exhibit a pronounced hysteresis, Nat. Struct. Biol. 2002, 9(2), $144-151$.

[58] D. Fasshauer, M. Margittai, A transient N-terminal interaction of SNAP-25 and syntaxin nucleates SNARE assembly, J. Biol. Chem. 2003, $279(9)$, 7613-7621.

[59] A. T. Brunger, K. M. Fiebig, L. M. Rice, E. Pollock, Folding intermediates of SNARE complex assembly, Nat. Struct. Biol. 1999, 6(2), 117-123.

[60] A. J. Kreutzberger, B. Liang, V. Kiessling, L. K. Tamm, Assembly and comparison of plasma membrane SNARE acceptor complexes, Biophys. J. 2016, $110(10), 2147-2150$.

[61] J. Genova, V. Vitkova, L. Aladgem, M. D. Mitov, Stroboscopic illumination gives new opportunities and improves the precision of bending elastic modulus measurements, J. Optoel. Adv. Mater 2005, 7, 257-260.

[62] I. Mey, M. Stephan, E. K. Schmitt, M. M. Müller, M. Ben Amar, C. Steinem, A. Janshoff, Local membrane mechanics of pore-spanning bilayers, J. Am. Chem. Soc. 2009, 131(20), 7031-7039.

[63] S. Steltenkamp, M. Müller, M. Deserno, C. Hennesthal, C. Steinem, A. Janshoff, Mechanical properties of pore-spanning lipid bilayers probed by scanning force microscopy, Biophys. J. 2006, 91(1), 217-226.

[64] J. W. Kuhlmann, I. P. Mey, C. Steinem, Modulating the lateral tension of solvent-free pore-spanning membranes, Langmuir 2014, 30(27), 8186-8192. 
[65] W. Breckenridge, I. Morgan, J. Zanetta, G. Vincendon, Adult rat brain synaptic vesicles II. lipid composition, BBA - General Subjects 1973, 320(3), 681-686.

[66] F. He, Laemmli-SDS-PAGE, Bio-Protocols 2011, 1 (11).

[67] M. Gleisner, Dissertation, Georg August University Göttingen, 2016.

[68] S. C. Stein, J. Thiart, TrackNTrace: A simple and extendable open-source framework for developing single-molecule localization and tracking algorithms, Sci. Rep. 2016, 6, 37947.

[69] G. Binning, C. Quate, Atomic force microscope, Phys. Lett. 1986, 56 (9), 930934.

[70] I. Mey, Dissertation, Johannes Gutenberg-Universität Mainz, 2009.

[71] A. Janshoff, M. Neitzert, Y. Oberdörfer, H. Fuchs, Force spectroscopy of molecular systems-single molecule spectroscopy of polymers and biomolecules, Angew. Chem. 2000, 39(18), 3212-3237.

[72] Y. F. Dufrene, P. Hinterdorfer, Recent progress in AFM molecular recognition studies, Pflügers Arch. 2007, 456(1), 237-245.

[73] J. E. Sader, I. Larson, P. Mulvaney, L. R. White, Method for the calibration of atomic force microscope cantilevers, Rev. Sci. Instrum. 1995, 66 (7), 3789-3798.

[74] J. L. Hutter, J. Bechhoefer, Calibration of atomic-force microscope tips, Rev. Sci. Instrum. 1993, 64(7), 1868-1873.

[75] R. W. Stark, T. Drobek, W. M. Heckl, Thermomechanical noise of a free vshaped cantilever for atomic-force microscopy, Ultramicroscopy 2000, 86(1-2), 207-215.

[76] M. Levy, R.; Maaloum, Measuring the spring constant of atomic force microscope cantilevers: thermal fluctuations and other methods, Nanotechnology 2001, $13(1), 33-37$.

[77] A. F. Stalder, T. Melchior, M. Müller, D. Sage, T. Blu, M. Unser, Low-bond axisymmetric drop shape analysis for surface tension and contact angle measurements of sessile drops, Colloids Surf., A 2010, 364(1-3), 72-81. 
[78] I. Mey, C. Steinem, A. Janshoff, Biomimetic functionalization of porous substrates: towards model systems for cellular membranes, J. Mater. Chem. 2012, 22(37), 19348.

[79] K. H. Sheikh, H. K. Christenson, R. J. Bushby, S. D. Evans, A model system to study the insertion of cholesterol into a phospholipid monolayer, J. Phys. Chem. 2007, 111, 379-386.

[80] J. Kozuch, C. Weichbrodt, D. Millo, K. Giller, S. Becker, P. Hildebrandt, C. Steinem, Voltage-dependent structural changes of the membrane-bound anion channel hVDAC1 probed by SEIRA and electrochemical impedance spectroscopy, Phys. Chem. Chem. Phys. 2014, 16, 9546-9555.

[81] A. Ulman, Formation and structure of self-assembled monolayers., Chem. Rev. 1996, $96(4), 1533-1554$.

[82] D. A. Offord, J. H. Griffin, Kinetic control in the formation of self-assembled mixed monolayers on planar silica substrates., Langmuir 1993, 9(11), 30153025 .

[83] C.-H. Huang, P.-Y. Hsiao, F.-G. Tseng, S.-K. Fan, C.-C. Fu, R.-L. Pan, Porespanning lipid membrane under indentation by a probe tip: a molecular dynamics simulation study, Langmuir 2011, 27 (19), 11930-11942.

[84] D. Norouzi, M. Müller, M. Deserno, How to determine local elastic properties of lipid bilayer membranes from atomic-force-microscope measurements: a theoretical analysis, Phys. Rev. E. 2006, 75, 061914.

[85] S. Gourianova, N. Willenbacher, M. Kutschera, Chemical force microscopy study of adhesive properties of polypropylene films: influence of surface polarity and medium, Langmuir 2005, 21(12), 5429-5438.

[86] C. E. D. Chidsey, G. Y. Liu, P. Rowntree, G. Scoles, Molecular order at the surface of an organic monolayer studied by low energy helium diffraction, $J$. Chem 1989, $91(7), 4421-4423$.

[87] N. Camillone, C. E. D. Chidsey, G.-y. Liu, G. Scoles, Superlattice structure at the surface of a monolayer of octadecanethiol self-assembled on $\mathrm{Au}(111), \mathrm{J}$. Chem. Phys. 1993, 98, 3503-3511. 
[88] C. A. Widrig, C. A. Alves, M. D. Porter, Scanning tunneling microscopy of ethanethiolate and n-octadecanethiolate monolayers spontaneously adsorbed at gold surfaces., J. Am. Chem. Soc. 1991, $113(8), 8$.

[89] C. Schoenenberger, J. A. M. Sondag-Huethorst, J. Jorritsma, L. G. J. Fokkink, What Are the Holes in Self-Assembled Monolayers of Alkanethiols on Gold?, Langmuir 1994, $10(3), 611-614$.

[90] B. Lundberg, S. Ekman, Mixed monolayers containing phosphatidylcholine, cholesterol, oleic acid, mono-and triolein., Acta Chem. Scand. B 1979, 33, 395-399.

[91] B. Lundberg, A surface film study of the lateral packing of phosphatidylcholine and cholesterol, Chem. Phys. Lipids 1982, 31(1), 23-32.

[92] E. Evans, D. Needham, Physical properties of surfactant bilayer membranes: thermal transitions, elasticity, rigidity, cohesion and colloidal interactions., $J$. Phys. Chem. 1987, 91(16), 4219-4228.

[93] D. Needham, R. Nunn, Elastic deformation and failure of lipid bilayer membranes containing cholesterol, Biophys. J. 1990, 58(4), 997-1009.

[94] M. Gleisner, I. Mey, M. Barbot, C. Dreker, M. Meinecke, C. Steinem, Driving a planar model system into the 3rddimension: generation and control of curved pore-spanning membrane arrays, Soft Matter 2014, 10(33), 6228-6236.

[95] C. G. Schuette, K. Hatsuzawa, M. Margittai, A. Stein, D. Riedel, P. Kuster, M. Konig, C. Seidel, R. Jahn, Determinants of liposome fusion mediated by synaptic SNARE proteins, Proc. Natl. Acad. Sci. U.S.A. 2004, 101 (9), 28582863.

[96] Y. Xu, F. Zhang, Z. Su, J. A. McNew, Y.-K. Shin, Hemifusion in SNAREmediated membrane fusion, Nat. Struct. Mol. Biol. 2005, 12(5), 417-422.

[97] A. Cypionka, A. Stein, J. M. Hernandez, H. Hippchen, R. Jahn, P. J. Walla, Discrimination between docking and fusion of liposomes reconstituted with neuronal SNARE-proteins using FCS, Proc. Natl. Acad. Sci. USA 2009, 106(44), $18575-18580$. 
[98] E. Karatekin, J. Di Giovanni, C. Iborra, J. Coleman, B. O'Shaughnessy, M. Seagar, J. E. Rothman, A fast, single-vesicle fusion assay mimics physiological SNARE requirements, Proc. Natl. Acad. Sci. USA 2010, 107(8), 3517-3521.

[99] J. Diao, Z. Su, Y. Ishitsuka, B. Lu, K. S. Lee, Y. Lai, Y.-K. Shin, T. Ha, A single-vesicle content mixing assay for SNARE-mediated membrane fusion, Nat. Commun. 2010, 1(5), 1-6.

[100] J. W. Kuhlmann, M. Junius, U. Diederichsen, C. Steinem, SNARE-mediated single-vesicle fusion events with supported and freestanding lipid membranes, Biophy. J. 2017, 112(11), 2348-2356.

[101] J. J. Sieber, K. I. Willig, C. Kutzner, C. Gerding-Reimers, B. Harke, G. Donnert, B. Rammner, C. Eggeling, S. W. Hell, H. Grubmüller, Anatomy and dynamics of a supramolecular membrane protein cluster, Science 2007, 317(5841), 10721076.

[102] K. Bacia, C. G. Schuette, N. Kahya, R. Jahn, P. Schwille, SNAREs prefer liquid-disordered over-raft- (liquid-ordered) domains when reconstituted into giant unilamellar vesicles, J. Biol. Chem. 2004, $279(36), 37951-37955$.

[103] M. Przybylo, J. Sýkora, J. Humpoličková, A. Benda, A. Zan, M. Hof, Lipid diffusion in giant unilamellar vesicles is more than 2 times faster than in supported phospholipid bilayers under identical conditions, Langmuir 2006, 22(22), 90969099.

[104] P. Reineck, D. Gómez, S. H. Ng, M. Karg, T. Bell, P. Mulvaney, U. Bach, Distance and wavelength dependent quenching of molecular fluorescence by Au@SiO2 core-shell nanoparticles, ACS Nano 2013, 7(8), 6636-6648.

[105] Y. S. Chi, H. R. Byon, B. S. Lee, B. Kong, H. C. Choi, I. S. Choi, Polymeric rulers: distance-dependent emission behaviors of fluorophores on flat gold surfaces and bioassay platforms using plasmonic fluorescence enhancement, Adv. Funct. Mater. 2008, 18(21), 3395-3402.

[106] P. Jönsson, M. P. Jonsson, J. O. Tegenfeldt, F. Höök, A method improving the accuracy of fluorescence recovery after photobleaching analysis, Biophys. J. 2008, 95 (11), 5334-5348. 
[107] M. L. Wagner, L. K. Tamm, Reconstituted syntaxin1A/SNAP25 interacts with negatively charged lipids as measured by lateral diffusion in planar supported bilayers, Biophys. J. 2001, 81(1), 266-275.

[108] V. Kiessling, S. Ahmed, M. Domanska, M. Holt, R. Jahn, L. Tamm, Rapid fusion of synaptic vesicles with reconstituted target SNARE membranes, Biophys. J. 2013, 104 (9), 1950-1958.

[109] F. Savic, Simulation von FRAP-experimenten, Theorie und Design von FRAPExperimenten auf komplexen Geometrien 2015, 1, 23-48.

[110] H.-C. Chiang, W. Shin, W.-D. Zhao, E. Hamid, J. Sheng, M. Baydyuk, P. J. Wen, A. Jin, F. Momboisse, L.-G. Wu, Post-fusion structural changes and their roles in exocytosis and endocytosis of dense-core vesicles, Nat. C 2014, 5, 1-15.

[111] A. Anantharam, B. Onoa, R. H. Edwards, R. W. Holz, D. Axelrod, Localized topological changes of the plasma membrane upon exocytosis visualized by polarized TIRFM, J. Cell Biol. 2010, 188(3), 415-428.

[112] D. L. Floyd, S. C. Harrison, A. M. van Oijen, Analysis of kinetic intermediates in single-particle dwell-time distributions, Biophys. J. 2010, 99(2), 360-366.

[113] M. Ollivon, S. Lesieur, C. Grabielle-Madelmont, M. Paternostre, Vesicle reconstitution from lipid-detergent mixed micelles, BBA - Biomembranes 2000, 1508(1-2), 34-50.

[114] M. M. Parmar, K. Edwards, T. D. Madden, Incorporation of bacterial membrane proteins into liposomes: factors influencing protein reconstitution, $B B A$ Biomembranes 1999, 1421(1), 77-90.

[115] A. Varnier, F. Kermarrec, I. Blesneac, C. Moreau, L. Liguori, J. L. Lenormand, N. Picollet-Dhahan, A simple method for the reconstitution of membrane proteins into giant unilamellar vesicles, J. Membr. Biol. 2010, $233(1-3), 85-92$.

[116] M. Fuhrmans, M. Müller, Mechanisms of vesicle spreading on surfaces: coarsegrained simulations, Langmuir 2013, 29 (13), 4335-4349.

[117] R. Jahn, T. C. Südhof, Synaptic vesicles and exocytosis., Annu. Rev. Neurosci. 1994, 17, 219-246. 
[118] F. J. Alenghat, D. E. Golan, Membrane protein dynamics and functional implications in mammalian cells, Curr. Top. in Membr. 2013, 1, 89-120.

[119] K. Weiß, A. Neef, Q. Van, S. Kramer, I. Gregor, J. Enderlein, Quantifying the diffusion of membrane proteins and peptides in black lipid membranes with 2-focus fluorescence correlation spectroscopy, Biophys. J. 2013, 105 (2), 455-462.

[120] J. J. Sieber, K. I. Willig, R. Heintzmann, S. W. Hell, T. Lang, The SNARE motif is essential for the formation of syntaxin clusters in the plasma membrane, Biophys. J. 2006, $90(8)$, 2843-2851.

[121] L. Mayer, M. Hope, P. Cullis, Vesicles of variable sizes produced by a rapid extrusion procedure, BBA - Biomembranes 1986, 858(1), 161-168.

[122] S. Martens, H. T. McMahon, Mechanisms of membrane fusion: disparate players and common principles, Nat. Rev. Mol. Cell Biol. 2008, 9(7), 543-556.

[123] C. A. Keller, K. Glasmästar, V. P. Zhdanov, B. Kasemo, Formation of supported membranes from vesicles, Phys. Rev. Lett. 2000, 84 (23), 5443-5446.

[124] L. Tamm, H. McConnell, Supported phospholipid bilayers, Biophys. J. 1985, $47(1), 105-113$.

[125] R. Jordan, E. A. Lemke, J. Klingauf, Visualization of synaptic vesicle movement in intact synaptic boutons using fluorescence fluctuation spectroscopys, Biophys. J. 2005, $89(3), 2091-2102$.

[126] C. Yoshina-Ishii, Y.-H. M. Chan, J. M. Johnson, L. A. Kung, P. Lenz, S. G. Boxer, Diffusive dynamics of vesicles tethered to a fluid supported bilayer by single-particle tracking, Langmuir 2006, 22(13), 5682-5689.

[127] J. J. Benkoski, F. Höök, Lateral mobility of tethered vesicle-DNA assemblies, J. Phys. Chem. B 2005, $109(19), 9773-9779$.

[128] J. M. Hernandez, A. J. B. Kreutzberger, V. Kiessling, L. K. Tamm, R. Jahn, Variable cooperativity in SNARE-mediated membrane fusion, Proc. Natl. Acad. Sci. USA 2014, $111(33), 12037-12042$.

[129] R. Mohrmann, H. de Wit, M. Verhage, E. Neher, J. B. Sorensen, Fast vesicle fusion in living cells requires at least three SNARE complexes, Science 2010, 330 (6003), 502-505. 
[130] C. Montecucco, G. Schiavo, S. Pantano, SNARE complexes and neuroexocytosis: how many, how close?, Trends Biochem. Sci. 2005, 30(7), 367-372.

[131] M. Böcker, S. Muschter, E. K. Schmitt, C. Steinem, T. E. Schäffer, Imaging and patterning of pore-suspending membranes with scanning ion conductance microscopy, Langmuir 2009, 25 (5), 3022-3028.

[132] X. Lu, Membrane fusion induced by neuronal SNAREs transits through hemifusion, J. Biol. Chem. 2005, $280(34)$, 30538-30541.

[133] T. Liu, T. Wang, E. R. Chapman, J. C. Weisshaar, Productive hemifusion intermediates in fast vesicle fusion driven by neuronal SNAREs, Biophys. J. 2008, $94(4), 1303-1314$.

[134] J. Diao, Y. Ishitsuka, H. Lee, C. Joo, Z. Su, S. Syed, Y.-K. Shin, T.-Y. Yoon, T. Ha, A single vesicle-vesicle fusion assay for in vitro studies of SNAREs and accessory proteins, Nat. Protoc. 2012, 7(6), 921-934.

[135] C. G. Giraudo, C. Hu, D. You, A. M. Slovic, E. V. Mosharov, D. Sulzer, T. J. Melia, J. E. Rothman, SNAREs can promote complete fusion and hemifusion as alternative outcomes, J. Cell Biol. 2005, 170(2), 249-260.

[136] A. Elhamdani, Double patch clamp reveals that transient fusion (kiss-and-run) is a major mechanism of secretion in calf adrenal chromaffin cells: high calcium shifts the mechanism from kiss-and-run to complete fusion, J. Neurosci. 2006, 26(11), 3030-3036.

[137] B. van Lengerich, R. J. Rawle, P. M. Bendix, S. G. Boxer, Individual vesicle fusion events mediated by lipid-anchored DNA, Biophys. J. 2013, 105(2), 409-419.

[138] Y. Kozlovsky, L. V. Chernomordik, M. M. Kozlov, Lipid intermediates in membrane fusion: formation, structure, and decay of hemifusion diaphragm, Biophys. J. 2002, 83(5), 2634-2651.

[139] W.-D. Zhao, E. Hamid, W. Shin, P. J. Wen, E. S. Krystofiak, S. A. Villarreal, H.-C. Chiang, B. Kachar, L.-G. Wu, Hemi-fused structure mediates and controls fusion and fission in live cells, Nature 2016, 534 (7608), 548-552.

[140] B. S. Stratton, J. M. Warner, Z. Wu, J. Nikolaus, G. Wei, E. Wagnon, D. Baddeley, E. Karatekin, B. OShaughnessy, Cholesterol increases the openness of SNAREmediated flickering fusion pores, Biophys. J. 2016, 110(7), 1538-1550. 
[141] P. J. Patty, B. J. Frisken, The pressure-dependence of the size of extruded vesicles, Biophys. J. 2003, 85(2), 996-1004.

[142] S. O. Rizzoli, R. Jahn, Kiss-and-run, collapse and 'readily retrievable' vesicles, Traffic 2007, 8(9), 1137-1144.

[143] A. Sonnleitner, G. Schütz, T. Schmidt, Free brownian motion of individual lipid molecules in biomembranes, Biophys. J. 1999, 77(5), 2638-2642.

[144] E. Sackmann, Supported membranes: scientific and practical applications., Science 1996, 271, 271.

[145] A. Grafmüller, J. Shillcock, R. Lipowsky, Pathway of membrane fusion with two tension-dependent energy barriers, Phys. Rev. Lett. 2007, 98(21), 218101.

[146] L. Gao, R. Lipowsky, J. Shillcock, Tension-induced vesicle fusion: pathways and pore dynamics, Soft Matter 2008, 4 (6), 1208-1214.

[147] J. Sun, Z. P. Pang, D. Qin, A. T. Fahim, R. Adachi, T. C. Südhof, A dual-Ca ${ }^{2+}$ sensor model for neurotransmitter release in a central synapse, Nature 2007, 450(7170), 676-682.

[148] B. L. Sabatini, W. G. Regehr, Timing of neurotransmission at fast synapses in the mammalian brain, Nature 1996, 384(6605), 170-172.

[149] D. Fasshauer, H. Otto, W. K. Eliason, R. Jahn, A. T. Brunger, Structural changes are associated with soluble N-Ethylmaleimide-sensitive fusion protein attachment protein receptor complex formation, J. Biol. Chem. 1997, $272(44)$, $28036-28041$.

[150] A. Stein, Dissertation, Max-Planck-Institut für Biophysikalische Chemie in Göttingen, 2007.

[151] A. Cypionka, Dissertation, Georg-August-Universität Göttingen, 2009.

[152] D. L. Floyd, J. R. Ragains, J. J. Skehel, S. C. Harrison, A. M. van Oijen, Single-particle kinetics of influenza virus membrane fusion, Proc. Natl. Acad. Sci. USA 2008, 105 (40), 15382-15387.

[153] Y. Gao, S. Zorman, G. Gundersen, Z. Xi, L. Ma, G. Sirinakis, J. E. Rothman, Y. Zhang, Single reconstituted neuronal SNARE complexes zipper in three distinct stages, Science 2012, 337(6100), 1340-1343. 
[154] F. Li, D. Kümmel, J. Coleman, K. M. Reinisch, J. E. Rothman, F. Pincet, A half-zippered SNARE complex represents a functional intermediate in membrane fusion, J. Am. Chem. Soc. 2014, 136(9), 3456-3464.

[155] T. A. M. Bharat, J. Malsam, W. J. H. Hagen, A. Scheutzow, T. H. Sollner, J. A. G. Briggs, SNARE and regulatory proteins induce local membrane protrusions to prime docked vesicles for fast calcium-triggered fusion, EMBO reports 2014, $15(3), 308-314$.

[156] H. T. McMahon, M. M. Kozlov, S. Martens, Membrane curvature in synaptic vesicle fusion and beyond, Cell 2010, 140(5), 601-605.

[157] T. C. Südhof, Neurotransmitter release: the last millisecond in the life of a synaptic vesicle, Neuron 2013, 80 (3), 675-690.

[158] T. C. Südhof, The synaptic vesicle cycle, Annu. Rev. Neurosci. 2004, 27(1), 509-547.

[159] J. Rizo, C. Rosenmund, Synaptic vesicle fusion, Nat. Struct. Mol. Biol. 2008, $15(7), 665-674$.

[160] X. Han, Transmembrane segments of syntaxin line the fusion pore of $\mathrm{Ca}^{2+}$ triggered exocytosis, Science 2004, 304 (5668), 289-292.

[161] Y. Hua, R. H. Scheller, Three SNARE complexes cooperate to mediate membrane fusion, Proc. Natl. Acad. Sci. USA 2001, 98(14), 8065-8070. 



\section{Curriculum vitae}

\section{Personal data}

Name: Jan Wilhelm Kuhlmann

Date of birth: $\quad 27.07 .1986$

Place of birth: Düsseldorf, Germany

Citizenship: German

\section{Carreer}

since 12/2012 Ph.D. thesis at the Georg-August University, Göttingen (Prof. Dr. C. Steinem), title of the thesis: Modulation of lateral membrane tension

2010-2012 Graduate studies in chemistry at the Georg-August University, Göttingen, degree: Master of Science, title of the master thesis: Kraftspektroskopische Studie an porenüberspannenden Membranen.

2007-2010 Undergraduate studies in chemistry at the Georg-August University, Göttingen, degree: Bachelor of Science, title of the bachelor thesis: Einfluss der Goldschichtdicke auf die Eigenschaften porenüberspannender Membranen.

2004-2007 Chemisch-Technischer Assistent at the Heinrich-Hertz-Berufskolleg, Düsseldorf, Germany

2003-2006 Allgemeine Hochschulreife at Marie Curie Gymnasium, Neuss, degree: Abitur

\section{Scientific contributions}

\section{Peer-reviewed publications}

J. W. Kuhlmann, M. Junius, U. Diederichsen and C. Steinem, SNAREmediated single vesicle fusion events with supported and freestanding lipid membranes, Biophys. J. 2017, 112, 2348-2356. tension of solvent-free pore-spanning membranes, Langmuir 2014, 30, 8186-8192. 


\section{Oral presentation}

2014 International Workshop "Molecular Membrane Biophysics, Hünfeld", Tuning the lateral tension of pore-spanning lipid bilayers.

\section{Selected poster presentations}

2016 International Symposium 2016 "Biological Dynamics from Microscopic to Mesoscopic Scales, Grimma", SNARE-mediated membrane fusion on planar pore-spanning membranes SFB 803 Symposium 2014, Göttingen

Tuning membrane tension by using binary SAMs

2013 9th European Biophysics Congress, Lisbon

Modulating the lateral tension of pore-spanning membranes 


\section{Danksagung}

Prof. Dr. Claudia Steinem möchte ich für die Betreuung der Doktorarbeit und die fachlichen Diskussionen danken, die mir während dieser Arbeit sehr geholfen haben.

Prof. Dr. Tim Salditt danke ich herzlich für die spontane Übernahme des Zweitgutachtens sowie für eine spannende Kooperation im Rahmen des SFBs.

Bei Prof. Dr. Burkhard Geil möchte ich mich für die fachliche Unterstützung bei der Auswertung von Dockingzeiten bedanken.

An Ingo geht ein besonderer Dank, für die vielen wertvollen Hilfestellungen und Ideen während meiner Arbeit und für ein offenes Ohr bei allen Anliegen.

Meiner Masterstudentin Nelli möchte ich für die sorgfältige Bearbeitung des Themas Oberflächenfunktionalisierung danken. Es hat viel Spaß gemacht mal etwas völlig neues auszuprobieren.

Vielen Dank Jutta und Micha für die Unterstützung im Labor. Ohne Euch wäre so ein komfortables Arbeiten nicht möglich gewesen.

Ich danke allen meinen Korrekturlesern, die die größten Schnitzer in meiner Arbeit (hoffentlich) entdeckt haben.

Bei Marianne, Melanie und Dana möchte ich mich für die Unterstützung bei den vielen organisatorischen Dingen bedanken.

Lando danke ich für die schöne Zeit als Labornachbar und vor allem für die Einarbeitung in die SNARE-Isolierung und Fusion.

Martin, Johannes, Raphael und Stefan danke ich für die vielfältigen fachlichen aber auch privaten Unterhaltungen. Ein besonderer Dank geht an Martin für die wertvollen Tipps an der Spinning Disc.

Herzlichen Dank an alle Kollegen für die wirklich schöne Zeit im Arbeitskreis! Ob gemütliche Abende im Kaffeeraum, stattliche Feiern in der OC-Cafete oder das Bauen am Abholungswagen. Alles hat sehr viel Spaß gemacht und wäre ohne Euch nur halb so schön gewesen!

Meiner Familie und Miri danke ich für die Unterstützung auf dem Weg hierhin. 
